\title{
Improved Dark Energy Constraints from 100 New CfA Supernova Type Ia Light Curves
}

\author{
Malcolm Hicken ${ }^{1,2}$, W. Michael Wood-Vasey ${ }^{3}$, Stéphane Blondin ${ }^{4}$, Peter Challis ${ }^{1}$, Saurabh \\ Jha $^{5}$, Patrick L. Kelly ${ }^{6}$, Armin Rest ${ }^{2,7}$, Robert P. Kirshner ${ }^{1}$
}

\begin{abstract}
We combine the CfA3 supernova Type Ia (SN Ia) sample with samples from the literature to calculate improved constraints on the dark energy equation of state parameter, $w$. The CfA3 sample is added to the Union set of Kowalski et al. (2008) to form the Constitution set and, combined with a BAO prior, produces $1+w=0.013_{-0.068}^{+0.066}(0.11$ syst $)$, consistent with the cosmological constant. The CfA3 addition makes the cosmologically-useful sample of nearby SN Ia between 2.6 and 2.9 times larger than before, reducing the statistical uncertainty to the point where systematics play the largest role. We use four light curve fitters to test for systematic differences: SALT, SALT2, MLCS2k2 $\left(R_{V}=3.1\right)$, and MLCS2k2 $\left(R_{V}=1.7\right)$. SALT produces high-redshift Hubble residuals with systematic trends versus color and larger scatter than MLCS2k2. MLCS2k2 overestimates the intrinsic luminosity of SN Ia with $0.7<\Delta<1.2$. MLCS2k2 with $R_{V}=3.1$ overestimates host-galaxy extinction while $R_{V} \approx 1.7$ does not. Our investigation is consistent with no Hubble bubble. We also find that, after lightcurve correction, SN Ia in Scd/Sd/Irr hosts are intrinsically fainter than those in $\mathrm{E} / \mathrm{S} 0$ hosts by $2 \sigma$, suggesting that they may come from different populations. We also find that SN Ia in Scd/Sd/Irr hosts have low scatter (0.1 mag) and
\end{abstract}

\footnotetext{
${ }^{1}$ Harvard-Smithsonian Center for Astrophysics, Cambridge, MA 02138; mhicken, kirshner @ cfa.harvard.edu

${ }^{2}$ Department of Physics, Harvard University, Cambridge, MA 02138

${ }^{3}$ Department of Physics and Astronomy, University of Pittsburgh, Pittsburgh, PA 15260

${ }^{4}$ European Southern Observatory, D-85748 Garching, Germany

${ }^{5}$ Department of Physics and Astronomy, Rutgers, the State University of New Jersey, Piscataway, NJ 08854

${ }^{6}$ Kavli Institute for Particle Astrophysics and Cosmology, Stanford University, 382 Via Pueblo Mall, Stanford, CA 94305

${ }^{7}$ Cerro Tololo Inter-American Observatory (CTIO), Colina el Pino S/N, La Serena, Chile
} 
reddening. Current systematic errors can be reduced by improving SN Ia photometric accuracy, by including the CfA3 sample to retrain light-curve fitters, by combining optical SN Ia photometry with near-infrared photometry to understand host-galaxy extinction, and by determining if different environments give rise to different intrinsic SN Ia luminosity after correction for light-curve shape and color.

Subject headings: supernovae: general — cosmology: dark energy

\section{Introduction}

One of the limitations of supernova cosmology has been the relatively low number of cosmologically-useful nearby SN Ia. The paucity of nearby objects has caused the statistical uncertainties in measurements of time-independent dark energy to be on the same order as systematic uncertainties. For example, Kowalski et al. (2008) use 250 SN Ia at high redshift but only 57 at low redshift. As part of their study, they add eight new nearby SN Ia light curves and find that their inclusion helps reduce the statistical uncertainty in the measurement of the cosmological constant. In this paper, we combine the latest sample

of nearby SN Ia optical photometry from the CfA SN Group (CfA3 sample) (Hicken et al. 2009, hereafter, H09) with samples from the literature and use multiple light-curve fitters to calculate dark energy values. The CfA3 sample consists of 185 objects, compared with 29 from the Calan-Tololo survey (Hamuy et al. 1996a), 22 from the "CfA1" sample (Riess et al. 1999), and 44 from the "CfA2" sample Jha et al. (2006). As many as 133 are above $z=0.01$ and are useful for dark energy calculations, depending on what cuts are made. This increases the number of cosmologically-useful nearby SN Ia by a factor of roughly 2.6-2.9 and reduces the statistical uncertainties of time-independent dark energy to the point where the largest uncertainties that remain are systematic. Because of these systematic uncertainties, we do not claim than any of our dark energy values is the "right" one. However, as the systematic errors (some of which are identified and addressed in this paper) are reduced in future studies, the promise of constraining cosmology from SN Ia with high precision will become more real. The task of solving the systematic problems that limit SN cosmology will be challenging but it is the most important area to focus on.

There are two main sources of known systematic uncertainty in SN cosmology. One is in the photometry itself. For example, good nearby light curves only have a typical accuracy of $0.03 \mathrm{mag}$ (as opposed to a precision of $0.015 \mathrm{mag}$ ) (see H09) and different groups' nearby samples disagree in their mean Hubble residuals by about 0.03 mag (see $\S 3.12$ herein). The second is the method of SN Ia distance estimation, typically involving corrections for light- 
curve shape and color to obtain the absolute intrinsic SN Ia magnitude. The main source of uncertainty here is how to treat host-galaxy reddening and disentangle this from intrinsic SN Ia color variation. A third source could also be that there are different populations of SN Ia that are equivalent in light-curve shape and color but actually have slightly different intrinsic luminosities.

The CfA3 sample is useful in identifying and reducing systematic errors on the photometric front because it provides a large, homogeneously-reduced and nearly homogeneouslyobserved nearby sample. The CfA3 sample can be used on its own as the nearby component and thus reduce the effects of systematic offsets with other samples. Also, the same photometric reduction pipeline that was used for the ESSENCE survey (Miknaitis et al. 2007) was used for the CfA3 sample, reducing one source of systematic uncertainty between nearby and faraway SN Ia. Regarding light-curve fitting and distance estimation methods, the CfA3 sample can be added to existing training sets to improve their accuracy and precision, especially since the inclusion of the slightly less-common slow and fast decliners was emphasized. For the first time we are in a position to examine different fitting methods on a large sample that was not used to train them. With the larger sample, light curve fitters can be trained better and a proper prediction error can be calculated by excluding individual objects (or groups of objects) from the training sample one at a time. Mandel et al. (2009) have developed the machinery for this in the near infrared and will be including the optical bands shortly. Additionally, many of the CfA3 objects were also observed spectroscopically (Matheson et al. 2008; Blondin et al. 2009) and photometrically in the near infrared (Wood-Vasey 2008; Friedman et al. 2009). The combination of the optical and near-infrared photometry should help disentangle host reddening from intrinsic SN Ia color and reduce this source of systematic error. Finally, the larger sample assists the search for different SN Ia populations, perhaps by host-galaxy morphology (see §4.2) or by host-galaxy color (Kelly et al. 2009).

Due to the light-curve-shape/luminosity relationship (broader brighter, narrower fainter), the natural scatter in intrinsic SN Ia luminosity is reduced by a factor of three. This makes SN Ia into standardizable candles and the most useful distance indicators at cosmological scales (e.g. Phillips 1993; Hamuv et al. 1996a; Riess. Press. \& Kirshner 1996; Perlmutter et al. 1997; Jha et al. 1999; Goldhaber et al. 2001; Guy et al. 2005; Jha, Riess, \& Kirshner 2007; Guy et al. 2007; Conley et al. 2008). They have been the key element in the discovery that the universe is accelerating and dominated by dark energy (e.g., Riess et al. 1998; Perlmutter et al. 1999; Knop et al. 2003; Tonry et al. 2003; Barris et al. 2004; Astier et al. 2006; Riess et al. 2007; Wood-Vasey et al. 2007; Davis et al. 2007; Kowalski et al. 2008). Observational efforts have moved beyond merely establishing the existence of dark energy and are focused on determining its simplest properties. This is most often done in terms of the 
equation of state, $p=w \rho$, where the equation of state parameter, $w$, relates the dark energy density, $\rho$, to the dark energy pressure, $p$. In a Friedman universe, $\rho$ depends on $1+w$ and the scale factor of the universe, $a$, as $\rho \sim a^{-3(1+w)}$. The first question that arises is whether the dark energy density is constant $(1+w=0$, a cosmological constant) or not. We choose to use the notation, $1+w$, since it is then easier to think about values of $w$ larger than -1 $(1+w>0)$ or more negative than $-1(1+w<0)$. In the case of $1+w<0$ the dark energy grows in density as the universe expands! The second question is whether the dark energy properties, as described by $w$, are constant in time or not.

The first study on the equation of state produced a 95\%-confidence limit of $1+w<0.3$, assuming $\Omega_{M} \sim 0.2$ and zero possibility of $1+w<0$ (Garnavich et al. 1998). Knop et al. (2003) found $1+w=-0.05_{-0.20}^{+0.15}$. Riess et al. (2005) reported $1+w=-0.02_{-0.19}^{+0.13}$. The SNLS and ESSENCE surveys were designed to narrow the constraints on $1+w$ and their first reports showed significant improvement in statistical uncertainty over the previous values, bringing them down to the range where systematic uncertainties, which they try to reduce as well, are of roughly equal importance. Astier et al. (2006, A06, hereafter) found $1+w=-0.02 \pm 0.09$ while Wood-Vasey et al. (2007, WV07, hereafter) found $1+w=-0.07 \pm 0.09$. Most recently, Kowalski et al. (2008) (K08, hereafter) made a compilation of the literature SN Ia, plus several new nearby ones that they present, and found $1+w=-0.01 \pm 0.08$ when using the same priors as A06 and WV07. All of these studies are consistent with a cosmological constant.

On the time-evolution of $1+w$, Riess et al. (2007) rule out rapidly evolving dark energy. Below $z \sim 0.4,1+w$ does not vary. However, large numbers of new high-redshift SN Ia $(z \sim 1.5)$ are needed to provide meaningful constraints on the long-term time evolution of $1+w$. Our focus is solely on time-independent $1+w$ (which we will just refer to as $1+w$ ), in a flat universe, where adding nearby SN Ia makes a significant improvement. We invite others to use the distances presented in this work and explore a wider range of dark energy models than we do.

We now summarize the present numbers of cosmologically-useful SN Ia light curves and some of the significant surveys that will be completed or operational within seven or eight years from now. The future estimates are largely drawn from the Report of the Dark Energy Task Force (Albrecht et al. 2006) and the FoMSWG (Figure of Merit Scientific Working Group) findings (Albrecht et al. 2009). Including the CfA3 sample, there are currently 150200 nearby SN Ia light curves $(z<0.15)$ that are useful for dark energy calculations. The KAIT sample, with 100 SN Ia, will soon be published, as will the Carnegie Supernova Project sample, also with $\sim 100 \mathrm{SN}$ Ia. Some of these are not in the Hubble flow and a significant number of these objects were also observed in the CfA2 and CfA3 surveys, so 
not all of the KAIT and Carnegie objects will be unique additions. In total, when these are included, there will be roughly 300 nearby SN Ia light curves that are useful for dark energy calculations. The Nearby Supernova Factory will also be presenting 300 spectrophotometric SN Ia light curves $(0.03<z<0.08)$ at some point. At a more nearby-to-intermediate redshift range, SDSS will soon publish a total of several hundred SN Ia light curves $(0.05<z<0.35)$.

From the compilation of K08, there are roughly 40 SN Ia from the High-Z team and 40 from the Supernova Cosmology Project, mostly in the redshift range $(0.3 \lesssim z \lesssim 1.2)$. ESSENCE has contributed $\sim 100$ SN Ia $(0.2<z<0.8)$ and will soon reach a total of $\sim 200$ with spectroscopic identification. About seventy percent of the ESSENCE SN Ia are useful for dark energy calculations. SNLS has published $\sim 75$ cosmologically-useful SN Ia light curves $(0.2<z<0.9)$ and will soon have a total of $\sim 500$. There are also $\sim 25$ SN Ia from the Higher-Z team, many above $z=1$.

All of these soon-to-be-published samples will drive statistical uncertainties of timeindependent dark energy about as low as is possible with the current state of SN Ia photometry and distance fitting. Further progress will only be made by significantly reducing systematic uncertainties. These additional samples will also contribute to this.

Looking ahead, the Dark Energy Survey should aquire roughly 2000-3000 SN Ia light curves by $2014(0.3<z<0.8)$ and Pan-STARRS will observe several thousand per year $(0.2<z<1)$ but only a small percentage will be spectroscopically identified. If the Hubble Space Telescope refurbishment is successful then an additional 50-100 SN Ia with $z>1$ should be obtained. Farther into the future, the JDEM will observe $2000+$ SN Ia in the range, $0.3 \lesssim z \lesssim 1.7$ with the intention of better constraining the time variation of dark energy. The LSST will provide truly staggering numbers of SN Ia, on the order of $10^{5}$ per year, most with $z<0.7$ and a smaller portion extending out to $z=1.2$. The JWST will be able to study SN Ia beyond $z \approx 2$, giving further insight into the matter-dominated era of the universe. In the design of these surveys, emphasis should be placed on reducing systematic uncertainies, as opposed to simply acquiring more objects with the same level of accuracy as in previous surveys.

Returning to the matter at hand, the CfA3 sample was acquired from 2001-2008, on the F. L. Whipple Observatory 1.2m telescope, mostly using two cameras, the 4Shooter and Keplercam, as described in H09. A few SN Ia were observed with the Minicam. UBVRI filters were used on the 4 Shooter while $U B V r^{\prime} i$ ' filters were used on the Minicam and Keplercam. CfA3 comprises over 11500 observations while CfA1 has 1210 and CfA2 has 2190. H09 show relatively good agreement with previous samples of nearby SN Ia in the distribution of intrinsic color and host-galaxy extinction. However, CfA3 has a wider distribution in light-curve shape, in large part due to H09 giving higher priority to fast and slow decliners in order 
to fill in the population sampling of both brighter and fainter SN Ia. This is of particular value for retraining light-curve fitters as well as providing light-curve phase information for spectra of the objects in the CfA3 sample. The slow decliners are also a valuable addition since these are found relatively more often at high redshift.

As discussed in greater detail in H09, about two thirds of the CfA3 SN Ia were discovered by professionals and one third by amateurs. KAIT was the main single contributor with $46 \%$ of the objects. The median redshift of the CfA3 objects above $z=0.01$ is $z=0.03$. The discovery limiting magnitude was typically $\sim 19.5$ mag but objects with a peak magnitude fainter than $\sim 18.5 \mathrm{mag}$ were not observed. This effective limiting magnitude for the CfA3 sample captures the whole range of SN Ia intrinsic luminosities out to $z \approx 0.03$. Beyond that, the fainter SN Ia drop out. The CfA3 sample is not representative of the relative numbers of the underlying nearby SN Ia distribution because of the many selection effects that are part of discovery and follow-up observations.

Combining the CfA3 sample with high- and low-redshift samples from the literature improves the constraints on $1+w$. We use four light curve fitters to probe for consistency and systematic effects: SALT (Guy et al. 2005), SALT2 (Guy et al. 2007), MLCS2k2 (Jha, Riess, \& Kirshner 2007, J07, hereafter) with $R_{V}=3.1$ (MLCS31) and MLCS2k2 with $R_{V}=1.7$ (MLCS17). Even though the underlying MLCS2k2 algorithm is the same for MLCS17 and MLCS31, we refer to them as two "different" fitters for the sake of simplicity. It is important to note that none of these fitters has been trained on the light curves from the CfA3 sample and yet we find reasonably consistent results between the CfA3 and literature nearby samples. We limit ourselves to these fitters so that comparisons with K08, MW07, and A06 can be made. However, we encourage that other light-curve fitters be used with the aim of exploring and decreasing systematic errors. Examples are $\Delta m_{15}(B)$ (Phillips 1993; Phillips et al. 1999), stretch (Goldhaber et al. 2001), CMAGIC (Wang et al. 2003; Conley et al. 2006), dm15 (Prieto, Rest, \& Suntzeff 2006), and SiFTO (Conley et al. 2008). We also encourage the addition of appropriate CfA3 objects to the these fitters.

To correctly determine the properties of the dark energy, it is vital to have distances at high and low redshift that are accurate relative to one another. The dividing line between high and low redshift in this paper is $z=0.16$ for SALT, which is consistent with $z=$ 0.20 in K08 (there are no objects with $0.16<z<0.20$, and only one object, SN 1999ar, with $0.15<z<0.16$ ), and $z=0.15$ for the other fitters, consistent with WV07. In the concordance model, $\Omega_{M} \approx 0.3$ and $\Omega_{\Lambda} \approx 0.7$, the SN Ia at $z \approx 0.5$ are about 0.25 mag fainter (and hence farther) than they would be in a universe with only matter $\left(\Omega_{M} \approx 0.3\right.$ ). If new samples or analysis showed the $z \approx 0.5 \mathrm{SN}$ Ia to be even fainter (and farther), and the nearby sample remained unchanged, then this would imply greater $\Omega_{\Lambda}$ (assuming $1+w=0$ in a flat 
universe) or lower $1+w$ (with $1+w$ free to vary in a flat universe) than before. Similar effects would be seen if the nearby distances decreased (brighter objects), and the $z \approx 0.5$ distances remain unchanged.

In comparing nearby and faraway SN Ia distances there are two key components: the underlying nearby and faraway SN Ia populations that nature provides and the distance estimation methods that we provide. Ideally, the nearby and faraway SN Ia populations would be composed of identical objects and the methods of distance estimation (photometry and light-curve/distance fitting) would work perfectly across the whole SN Ia range.

There is good evidence that the faraway population is composed of objects highly similar to the part of the nearby sample that it overlaps with. Comparisons of high and low redshift spectra reveal good agreement (e.g., Matheson et al. 2005; Hook et al. 2005; Howell et al. 2005; Foley et al. 2005; Blondin et al. 2006; Foley et al. 2008). Howell et al. (2007) mimic there being a difference in faraway and nearby populations due to evolution by only using SN Ia with light curve stretch parameter, $s \geq 1$ at $z \geq 0.4$ and SN Ia with $s<1$ at $z<0.4$ to fit the cosmology and find it is consistent with the results of the full sample. We assume that the nearby and faraway population objects are sufficiently similar for our purposes.

This leaves us with the issue of whether the distance estimation is sufficiently accurate or not. It is vital to accurately handle any aspects of the distance estimation that are unique, or much more heavily weighted, to either the faraway or nearby samples, such as $K$-corrections for the faraway SN Ia or including underluminous fast decliners at low redshift. It is also key to limit the samples to the range where the distance estimators are accurate enough and the faraway and nearby subsamples overlap sufficiently in underlying population and sampling characteristics so that any remaining inaccuracies in the distance estimators do not give rise to any significant differences. One option is to remove any troublesome groups, potentially gaining systematic safety while losing statistical leverage.

An example of this is handling host galaxy reddening. There are more highly-reddened SN Ia in the nearby sample. If the host extinction is estimated too high, perhaps because the physical value of $R_{V}$ is less than is being assumed, then the nearby distances will be too low on average, resulting in an overestimated dark energy density. Until the issue of handling host reddening is improved it is probably advisable to cut out moderately- and highly-reddened objects, and we follow this course for our "best"-cut samples.

As SN Ia samples grow in size and quality it may not be statistically necessary to include less-reliable objects. However, it is also likely that improved data sets and distance estimation techniques will result in SN Ia being better standardizable candles so that a wider range of objects can be safely included. 


\subsection{Outline of the Paper}

In $\S 2$, we describe the SN Ia light curve samples and what initial "minimal" quality cuts we make to ensure that poorly-fit objects are not being used to calculate cosmological results. We also describe the four light-curve fitters we use to calculate distances and how we calculate the cosmological fits.

In $\S 3$, we present our cosmological fits and examine the impact of the CfA3 sample. It reduces the statistical error on $1+w$ by a factor of 1.2-1.3, slightly less than rough $\sqrt{N}$ statistics would imply, suggesting that systematic uncertainties are becoming noticeable. Our Constitution sample (Union+CfA3), fit by SALT and using linear color-luminosity and lightcurve-shape-luminosity relations, produces $1+w=0.013_{-0.068}^{+0.066}(0.11$ syst $)$ when combined with a BAO prior. This is consistent with the cosmological constant. We use the four lightcurve fitters and different SN Ia samples to test for consistency and systematic differences and find generally good agreement. An encouraging example is the excellent agreement of the distances to two SN Ia in the same galaxy ("twins") that was found using each of the four fitters. However, there are three areas of concern: SALT, and to a lesser extent, SALT2, produce a trend in Hubble residuals versus the color parameter, $c$, at high redshift; MLCS2k2 gives rise to mostly negative residuals in the moderately underluminous region $0.7<\Delta<1.2$; and $R_{V}=3.1$ seems to overestimate the host galaxy extinction, $A_{V}$, in MLCS31. In $§ 3.11$, we choose to remove these objects for our "best"-cut samples. With and without the best cuts, SALT and SALT2 produce values of $1+w$ that are statistically consistent with the cosmological constant. Without the best cuts, MLCS31 also is consistent with the cosmological constant while MLCS17 produces $1+w \approx 0.1$. After the best cuts, MLCS31 and MLCS17 agree much better with each other but are slightly more than $1 \sigma$ above the cosmological constant $(1+w=0)$. We believe that when the systematic problems we discuss are resolved in retrained or future fitters that there will be much better agreement amongst different fitters and a more accurate measurement of the dark energy will exist.

In $§ 3.12$, we break up the nearby sample into its six or seven principal subsamples, based on observing survey. We examine the implied consistency of the photometry and light-curve/distance fitting of the six-to-seven largest nearby samples by comparing their average Hubble residuals and find typical agreement to be roughly 0.03 mag. MLCS17 gives the best agreement between the samples $(\sim 0.02 \mathrm{mag})$, MLCS31 and SALT come next ( $0.03 \mathrm{mag})$, and SALT2 has the most discrepancy $(\sim 0.06 \mathrm{mag})$.

In $\S 4$, We explore the issue of the "Hubble bubble" and look for trends in Hubble residuals versus host-galaxy properties. Our results are largely consistent with no Hubble bubble. We find that the SN Ia in Scd/Sd/Irr hosts are fainter after light-curve correction by $2 \sigma$ than those in E/S0 hosts, suggesting that it may be advantageous for light-curve fitting 
to divide the SN Ia sample into two or more groups based on host-galaxy properties. We briefly comment on the systematic uncertainty in $1+w$, finding it to be roughly 0.11 mag and $\sim 40-70 \%$ larger than the statistical uncertainty. The largest systematics seem to be which nearby sample and which light curve fitter are used. The treatment of host reddening is the largest contributor to the uncertainty of a given fitter. Systematic uncertainties now play the largest role in limiting our understanding of dark energy. To reduce these, three main steps should be taken. First, future photometry needs to be more accurate, by better understanding the instrumental passbands and their absolute calibration. Second, light curve fitters need to be retrained with larger samples, including the CfA3 and other forthcoming samples, treating the intrinsically-red and subluminous SN-1991bg-like objects separately. And third, intrinsic SN Ia color and host-galaxy reddening, at both high and low redshift, need to be disentangled.

\section{Light Curve Fitters}

SALT and SALT2 are light curve fitters and require a two-step approach towards calculating distances: first, fit each light curve for peak magnitude, stretch parameter ( $s$ for SALT and $x_{1}$ for SALT2) and color, $c$; second, use these outputs to calculate the best-fit cosmology. Typically, the distance modulus is parameterized by a linear dependence on shape and color parameters and we follow this path. SALT and SALT2 do not attempt to disentangle intrinsic color from host reddening. To be valid for cosmological calculations, both the high and low redshift samples must obey the same, combined intrinsic-plus-host-reddening color-magnitude relations.

Using MLCS2k2 also takes two steps to find the best-fit cosmology: fit for the distances and then fit for the cosmology. MLCS2k2 goes farther than SALT and fits for the distance along with its shape/luminosty parameter, $\Delta$, the host-galaxy extinction parameter, $A_{V}$ and the time of $B$ maximum, $t_{o}$. MLCS2k2 differs from SALT and SALT2 in that it attempts to explicitly calculate the host reddening by employing a prior on $E(B-V)$ which can then be converted to the extinction $A_{V}$ via the reddening law $R_{V}$. We use $R_{V}=3.1$ and $R_{V}=1.7$, effectively producing two MLCS2k2 fitters. It also uses a quadratic dependence on the shape parameter $\Delta$ to describe all intrinsic variations of the peak magnitude. No explicit, intrinsic-color parameter is employed. Rather, the broader-bluer, narrower-redder relation is incorporated into $\Delta$ so that the negative- $\Delta$ light curve template is both blue and broad while the highly positive- $\Delta$ template is red and narrow.

A weakness of the second step of using SALT and SALT2 is giving the same color-

magnitude relation to both intrinsic SN Ia color variation and host-galaxy dust. A weakness 
of MLCS2k2 is the uncertain nature of the prior on $E(B-V)$, especially at high redshift, and the reddening law that should be used. The use of all four fitters assumes that SN Ia are intrinsically the same at high and low redshift for a given shape and color, within the observed scatter. The veracity of this assumption has been explored and seems to hold but needs further investigation.

Our main purpose for fitting with SALT is to add the CfA3 sample to the Union compilation of K08. We call the combination of the Union and the CfA3 sets the "Constitution" set, to form a more perfect union. For the other three fitters, we do not use all the high-redshift SN Ia samples, specifically excluding $\sim 100$ objects from the SCP and HighZ objects from Riess et al. (1998), Perlmutter et al. (1999), Knop et al. (2003), Tonry et al. (2003), and Barris et al. (2004). Rather, we use the ESSENCE (Miknaitis et al. 2007), SNLS (A06) and Higher-z samples (Riess et al. 2007) where we believe the systematics to be better controlled. We will call these three together the "High-z" sample. The low redshift SN Ia compiled in Jha 2006 (referred to hereafter as "OLD") and the CfA3 sample constitute our nearby, "OLD+CfA3," sample. For each fitter we separately combine the OLD, CfA3 and OLD+CfA3 samples with the High-z sample. We will use these three nearby sample names to refer to the three nearby plus High-z samples since the High-z sample is common to each. We will also use "OLD" to refer to the nearby sample and the nearby plus high-redshift sample in K08. In the SALT case, OLD is equivalent to Union and OLD+CfA3 is equivalent to Constitution.

In all our $\Omega_{M-}-w$ cosmology fits, we assume a flat universe and combine the baryonic acoustic oscillations (BAO) constraints on $\left(\Omega_{M}, 1+w\right)$ from Eisenstein et al. (2005) with the SN Ia fits to produce our best-fit cosmologies. The BAO prior provides an effective constraint on the range of $\Omega_{M}$, primarily, while the SN Ia data better constrain $1+w$. Together, they form a complementary and excellent pair of constraints on $\Omega_{M}$ and $1+w$. Additional priors, such as from the cosmic microwave background, could be applied to increase the precision of $1+w$ but we prefer to leave the SN Ia effects as unmasked as possible while still narrowing the range in $\Omega_{M}$. The uncertainty on redshift can be seen in Tables 1, 2, 3, and 4, A peculiar velocity uncertainty of $400 \mathrm{~km} \mathrm{~s}^{-1}$ is assumed. A06, J07, K08 and others have used $300 \mathrm{~km} \mathrm{~s}^{-1}$ while WV07 used $400 \mathrm{~km} \mathrm{~s}^{-1}$. A06, WV07 and K08 make redshift cuts at $z=0.015$. Even though $300 \mathrm{~km} \mathrm{~s}^{-1}$ is closer to the actual peculiar velocity uncertainty, since we choose to cut at $z=0.01$ and because of potential issues like a Hubble bubble we would like to deweight the nearest ones slightly and $400 \mathrm{~km} \mathrm{~s}^{-1}$ achieves this. The peculiar velocity uncertainty of $400 \mathrm{~km} \mathrm{~s}^{-1}$ produces a difference in distance-modulus uncertainty between $z=0.01$ and $z=0.015$ that is $0.011 \mathrm{mag}$ greater than if $300 \mathrm{~km} \mathrm{~s}^{-1}$ had been used. Our use of ' $z$ ' refers to $z_{c m b}$ throughout. We also fit for $\Omega_{M}$ and $\Omega_{\Lambda}$, assuming $1+w=0$ and using the BAO prior. 


\subsection{SALT: Augmenting the Union Compilation}

SALT uses a modified version of the Nugent spectral template that was developed to reproduce the $U B V R$ light curves of 34 nearby SN Ia, 28 of which are at $z<0.015$ and six others with good $U$-band data at $z>0.015$. No SN-1991bg-like objects were included in this training set. SN-1991bg-like objects are spectroscopically identifited by strong Ti II lines. Photometrically, they are intrinsically red, fast declining, and subluminous. H09 show that such objects form more of a separate grouping than a continued distribution in color and intrinsic luminosity. SALT fits for the time of $B$-band maximum light, $t_{o}$, the flux normalization (which can be converted to the rest-frame peak magnitude in $B$ band, $m_{B}^{\max }$ ), a time stretch factor, $s$, and a color parameter, $c=\left.(B-V)\right|_{t=B \max }+0.057$. The color, $c$, is a combination of the intrinsic color and reddening due to host-galaxy dust.

The next step, taking the SALT output parameters and calculating the best-fit cosmology uses the following equation:

$$
\mu_{B}=m_{B}^{\max }-M+\alpha(s-1)-\beta c
$$

The empirical coefficients $\alpha, \beta$, and $M$ are marginalized over as part of the cosmological fit. It is remarkably the case that the two different physical mechanisms (intrinsic color and reddening by dust) that give rise to the color, $c$, sufficiently obey the same color-magnitude relation that they can be described by the single parameter, $\beta$. To calculate the distances to each SN Ia, we use the 1-d marginalization coefficients, $\alpha, \beta$, and $M$.

K08 use SALT to fit 307 SN Ia light curves, 57 at $z<0.2$ and 250 at $z>0.2$ to form the Union compilation. These SN meet the criteria of having good light curve fits, $z \geq 0.015$, time of first observation (divided by stretch, $s$ ), $t_{1 s t} / s$, less than six days after maximum light and each Hubble residual, scaled by its uncertainty, within $3 \sigma$. The K08 cuts are different from those that we will use for the other three fitters. We tested the K08 cuts on the MLCS31 OLD+CfA3 sample and it only made a difference in $1+w$ of 0.005 so we prefer to use our more permissive cuts, explained below, to allow for a larger sample.

We take the SALT parameters, $m_{B}^{\max }, s$, and $c$, from Table 11 in K08 for the Union set and calculate the best-fit cosmology from the combination of the $307 \mathrm{SN}$ plus the BAO prior, assuming a flat universe. There can be slight differences in the specific procedure to fit for the cosmology so we do not try to exactly reproduce the values for $1+w$ and $\Omega_{M}$; only get very close so that we can then confidently add our SALT fits of the CfA3 sample to the K08 SALT fits of the Union sample and calculate the Constitution set (OLD+CfA3) cosmology. 
A peculiar velocity of $400 \mathrm{~km} \mathrm{~s}^{-1}$ is assumed (different than the $300 \mathrm{~km} \mathrm{~s}^{-1}$ in $\mathrm{K} 08$ since we want to be more cautious in our assumptions for peculiar velocities). In order to approximately maintain the same relative weighting for each SN that was used by K08, including a sample-dependent systematic error and other uncertainty components, we take the uncertainty of the distance modulus, $d \mu_{B}$, from Table 11 in K08 and subtract off the uncertainties of $c$ and $s$ and use this as a modified uncertainty for $m_{B}^{\max }$ in equation 1, We find that the resulting uncertainty on each $\mathrm{SN}$ is too high by $0.007 \mathrm{mag}$ (in quadrature) to reproduce the same amount of uncertainty in $1+w$ as in K08 and we subtract this from each. These steps allow us to reproduce the $\mathrm{SN}+\mathrm{BAO}$ value of $1+w=-0.011$ from K08. The statistical uncertainties on $1+w$ from our calculations are more symmetric: +0.078 and -0.080 versus +0.076 and -0.082 for K08 but the difference between the positive and negative uncertainties is 0.158 for both.

Having sufficiently reproduced the K08 results, the CfA3 sample is added to the Union set. To verify that the SALT outputs for the CfA3 sample can be combined with the Union set we run most of the light curves from the nearby Union sample through SALT, using the UBVR bands only. We only run SALT on the J07 compilation of nearby SN Ia and so a few of the Union nearby set are not used to verify that our SALT output is consistent with K08. Our calculations of $m_{B}^{\max }, s$, and $c$ agree sufficiently with those of Table 11 in K08, with our peak magnitude fit in $B$ band fainter by $0.01 \pm 0.06 \mathrm{mag}$. Possible reasons for the slight differences are that K08 perturbs the light curves of the more poorly-fit objects, there might be slightly different versions of SALT and some other parameters within SALT may differ. However, since the typical scatter in the Hubble diagram for nearby SN Ia is in the range of 0.14-0.20 mag, our SALT calculations agree well enough with K08 that the SALT fits of the CfA3 sample can be safely combined with the K08 SALT fits of the whole Union set without introducing any significant offset.

SALT is not suited to fitting $1991 \mathrm{bg}$-like SN so we do not include ones that we identified as such by their spectra in the CfA3 sample. We did not remove the two 1991bg-like SN from the Union set, however, since the number is small and we are interested in updating the Union set as is. The CfA3 UBVRr' band light curves were run through SALT. We wanted to appropriately weight the CfA3 objects so that they can be combined with the Union set for cosmology calculations. We use the SALT output from K08 for the Union set and our own SALT calculations for the CfA3 set. Although the CfA3 Hubble residuals have a lower standard deviation than the nearby Union residuals (0.164 mag versus $0.186 \mathrm{mag}$ ), we made the more cautious assumption that, on average, a CfA3 SN Hubble residual should have the same uncertainty as a nearby Union SN Hubble residual. To accomplish this, an uncertainty of $0.138 \mathrm{mag}$ is added in quadrature to the uncertainties of the SALT light-curve fit for each CfA3 SN. The peculiar velocity uncertainty is set at $400 \mathrm{~km} \mathrm{~s}^{-1}$ and the redshift uncertainty 
is 0.001. Out of 185 CfA3 SN Ia, 90 survive after applying the Union cuts (these are our minimal cuts for SALT), increasing the nearby sample with these cuts by a factor of 2.6, from 57 to 147 . We also fit for $\Omega_{M}$ and $\Omega_{\Lambda}$, assuming $1+w=0$ and using the BAO prior.

\subsection{SALT2}

SALT2 differs from SALT by no longer using the Nugent template. Hsiao et al. (2007) presents an improved SN Ia spectral template, but rather than using this, SALT2 has a colorand stretch-dependent model spectrum derived from a sample of nearby and faraway SN Ia spectra and light curves. The main benefit of this is that the model spectrum for a given phase, color, and stretch is more closely derived from actual SN Ia data and so, in principle, the range of SN Ia light curves should be better fit than in SALT. Output parameters of the fit are the color, $c$, the stretch factor, $x_{1}$ (analogous but not equivalent to $s$ in SALT), the $B$-band maximum, $m_{B}^{\max }$, and the time of $B$ maximum, $t_{B \max }$. A practical effect of using the high redshift spectra is that the rest-frame near UV can be modeled out to $2000 \AA$. As in SALT, 1991bg-like SN are not included in the training and should not be fit with SALT2.

We run SALT2 on the OLD, CfA3 and High-z samples, excluding $I$ and $i$ ' from the nearby samples. The $U$-band fits were typically poor but their contribution to the overall light-curve fit was small because the $U$-band is given significantly less weight in SALT2 than the other optical bands. In $\S 3.9$, we explore the effects on $1+w$ of excluding the rest $U$ band and find little difference for SALT2. We used the default setting of $3460 \AA$ for the lower limit on the wavelength range. The value used by Guy et al. (2007) is different: $2900 \AA$. This shorter wavelength limit may improve the precision of the color parameter, $c$ for the high-redshift objects, subject to the uncertainties in calibrating the rest-frame UV. We did not explore the effects of of varying the short wavelength limit. We also exclude any 1991bglike objects. The reduced chi-squared, $\chi_{\nu}^{2}$, of the light curve fit allows poorly-fit objects to be flagged and removed. The precise value of $\chi_{\nu}^{2}$ that indicates a poor fit, as determined by visual inspection, varies from fitter to fitter, depending largely on the uncertainty attributed to the model light curve produced. If the model light curve has reasonable uncertainties then a $\chi_{\nu}^{2}$ of one can be expected but if it is too small then acceptable fits can have higher $\chi_{\nu}^{2}$. For SALT2 we found that the poorly-fit light curves had a $\chi_{\nu}^{2}$ of more than 10 while sufficientlywell-fit light curves were less than this and so we chose this as our cut-off value. We also only include SN Ia with $t_{1 s t} \leq+10 d$, ensuring that the fit parameters can be well constrained. SN with $z<0.01$, where peculiar velocity uncertainties become excessively large, are excluded from our cosmology fit for SALT2. These are the minimal cuts for SALT2. An additional uncertainty of $0.158 \mathrm{mag}$ is required to give a $\chi_{\nu}^{2}$ of one for the nearby SN Ia in our SALT2 
cosmology fit. This is added to all objects in the fit, both nearby and faraway.

A similar procedure to the SALT cosmology fitting is followed with the SALT2 output. The distance modulus is

$$
\mu_{B}=m_{B}^{\max }-M+\alpha x_{1}-\beta c
$$

and $\alpha, \beta$ and $M$ are marginalized over to find the best-fit cosmology. To calculate the distances to each SN Ia, we use the 1-d marginalization values for the coefficients, $\alpha, \beta$, and $M$.

\section{3. $\quad$ MLCS2k2 $\left(R_{V}=3.1\right)$}

MLCS2k2 consists of two main components: a model light curve parameterized by $\Delta$ and a physically-inspired attempt at separating instrinsic color variation from host-galaxy dust extinction. The intrinsic light curve shapes, color and luminosity of the model are a function of the single parameter $\Delta$. Although MLCS2k2 fits for all of its parameters simultaneously, it is conceptually useful to think of MLCS2k2 in the following manner. In calculating distances, MLCS2k2 assumes that a SN Ia can be corrected to the absolute magnitude of the fiducial SN Ia by means of the linear and quadratic terms in $\Delta$. The best-fit $A_{V}$ is added and the distance modulus, $\mu$, is the remaining term needed to reach the ( $K$ - and Milky Way-reddening-corrected) apparent magnitude. Obtaining an accurate value of $A_{V}$ depends in part on using the correct value of $R_{V}$. If $R_{V}$ is too high then $A_{V}$ will be as well and the distance modulus will be too small and vice versa. 1991bg-like SN Ia are part of the MLCS2k2 training sample, making a significant contribution to the model colors. This, in turn, has an impact on the extinction that is measured. To run these objects through MLCS2k2 correctly, $K$-corrections with a 1991bg-like spectral model are used.

MLCS2k2 was trained on a large number of high-quality, nearby SN Ia light curves where the host-galaxy extinction can be accurately estimated from well-sampled late-time $B$ and $V$ data and where the distance can be accurately estimated by only using Hubble flow objects. For nearby samples, the prior should be calculated from the sample itself or from a similar one. MLCS2k2 uses a one-sided exponential of scale-length $\tau_{E(B-V)}=0.138$ mag as a prior on host reddening. We refer to this color-excess prior as the "default" prior and use this for our nearby MLCS2k2 prior. For faraway samples, the prior is difficult or impossible to calculate from the data itself, due to the lack of late-time photometry. In this case, a nearby or a theoretical prior can be modified to match the detection effects for a sample where high-extinction events are less and less likely to be found at higher and 
higher redshifts. For example, the ESSENCE team modifies the Galactic Line of Sight (glos) prior (Riess et al. 1998; Hatano, Branch, \& Deaton 1998; Commins 2004; Riess et al. 2005; Riello \& Patat 2005) into the redshift-dependent "glosz" to take into account the redshiftdependent detection probabilities of their survey (WV07).

We run the SN Ia through MLCS2k2. We use the 1991bg-appropriate $K$-corrections for 28 objects that were identified as having 1991bg-like spectral properties: SN 1986G, 1991bg, 1992K, 1992bo, 1993H, 1997cn, 1998bp, 1998de, 1999by, 1999da, 1999gh, 2002fb, 2003D, $2005 \mathrm{ke}, 2005 \mathrm{mz}, 2006 \mathrm{H}, 2006 \mathrm{bd}, 2006 \mathrm{bz}, 2006 \mathrm{cs}, 2006 \mathrm{em}, 2006 \mathrm{gt}, 2006 \mathrm{hb}, 2006 \mathrm{je}, 2006 \mathrm{ke}$ 2007N, 2007al, 2007ax, and 2007ba. For the nearby sample we use the default host-galaxy reddening prior, assuming that the CfA3 sample is close enough to the sample from which the prior was derived. We use the glosz prior for the ESSENCE sample and the modified glosz prior for the SNLS light curves, both as described in WV07. For the Higher-z SN, we also use a modified glosz prior as described in Riess et al. (2005). We restrict our nearby sample to $z \geq 0.01$. Adding an "intrinsic" uncertainty of 0.078 mag produces a $\chi_{\nu}^{2}$ of one in the cosmological fit for the nearby sample and this is applied to the high redshift SN as well. Only those SNe whose MLCS31 fits have a $\chi_{\nu}^{2}$ of 1.5 or less are included. Additional criteria for inclusion are $A_{V} \leq 1.5$ and $t_{1 s t} \leq 10 d$. These are the minimal cuts for MLCS31.

\section{4. $\quad$ MLCS2k2 $\left(R_{V}=1.7\right)$}

Previous studies that used stretch or SALT have found the color parameter $\beta$ to be around 2, significantly lower than the value of 4.1, expected if $c$ were only measuring the host-galaxy dust and this dust obeys $R_{V}=3.1$, the reddening law seen in the Milky Way (e.g., Astier et al. 2006; Conley et al. 2007). However, it is unlikely that $c$ is only the color excess due to dust. Therefore, it is not surprising that typical values of $\beta$ are different than 4.1, rather that they are so much less. With this in mind, we use MLCS2k2 to find the value of $R_{V}$ that minimizes the scatter in the Hubble residuals for the nearby CfA3 sample. We run MLCS2k2 with no prior on the color excess $E(B-V)$, allowing for negative values as well. We calculate the Hubble residuals versus $A_{V}$ for both $R_{V}=3.1$ and 2.1. With $R_{V}=3.1$ and no prior there is a significant slope while for $R_{V}=2.1$ it is less severe. We subtract off the $A_{V}$ calculated for each object and fit for the effective $R_{V}$ that minimizes the scatter: $R_{V}=1.7$ is this value.

We fix $R_{V}$ to 1.7 and run MLCS2k2 on our compilation of SN Ia, using the same priors as in the case of $R_{V}=3.1$. We used a version of MLCS2k2 that has model UBVRI light curves that were calculated by training MLCS2k2 with $R_{V}=1.9$. We found it made little difference to the end results whether we used the version trained with $R_{V}=3.1$ or $R_{V}=1.9$ 
and so we did not bother to train with $R_{V}=1.7$, but we did use the $R_{V}=1.9$ version to be as consistent as possible.

We restrict our sample to $z \geq 0.01$. We use the $1991 \mathrm{bg}$-appropriate $K$-corrections for the same $\mathrm{SN}$ as we did with $R_{V}=3.1$. An intrinsic uncertainty of 0.083 mag is added to produce a $\chi_{\nu}^{2}$ of one in the nearby sample. Once again, we cut out objects with $A_{V} \geq 1.5$ and $t_{1 s t}>10 d$ days. The uncertainties in the model light curves trained with $R_{V}=1.9$ are slightly different than the $R_{V}=3.1$ case and so we choose to cut out SN with MLCS2k2 fits with $\chi_{\nu}^{2}$ greater than 1.6. This is the only difference in the minimal cuts between MLCS17 and MLCS31.

\section{Results and Systematics Due to Fitters and Nearby Samples}

This section analyzes the output of the four light curve fitters being employed for different subsamples, looking for systematic trends and errors in both fitters and SN Ia subsamples. The aim is to find areas where these fitters work best and provide suggestions for improvement. We also want to see how consistent the various nearby samples (from different groups and instruments) are with each other.

\subsection{SALT-The Constitution Sample}

We show the Hubble diagram and residuals with respect to an $\left(\Omega_{M}=0.27, \Omega_{\Lambda}=0\right)$ universe in Figure 1, with the Constitution sample best-fit cosmology overplotted. For comparison, a similar plot, but for MLCS17, is shown in Figure2, One of the main differences is the high scatter at high redshift in the SALT Hubble residuals versus the lower scatter at high redshift in MLCS17 (and MLCS31). In Figure 3, we also plot the $\left(\Omega_{\Lambda}, \Omega_{M}\right)$ probablity contours from the $\left(\Omega_{\Lambda}, \Omega_{M}, 1+w=0\right)$ fit for both the SN-only fits and the SN+BAO fits for the Union and Constitution sets. The SN-only contours become tighter along the $\Omega_{\Lambda}$ axis when the CfA3 sample is added. Adding the BAO prior gives $\Omega_{\Lambda}=0.718_{-0.056}^{+0.062}$ and $\Omega_{M}=0.281_{-0.016}^{+0.037}$.

Regarding $1+w$, as described in $\S 2.1$, the SALT fits on the Union sample, combined with the BAO prior, give $1+w=-0.011_{-0.080}^{+0.078}$, with 57 nearby and 250 High-z SN Ia used. When we only use the 90 CfA3 objects that pass the Union cuts with the 250 High-z SN Ia then we get $1+w=-0.002_{-0.075}^{+0.073}$. The CfA3 sample, after applying the Union cuts, is 1.58 times larger than the Union nearby sample. When we combine the 90 CfA3 SN Ia with the 57 OLD and 250 High-z Union SN Ia sample to form the Constitution sample we 
get $1+w=0.013_{-0.068}^{+0.066}$. This decreases the uncertainty in $1+w$ by a factor of 1.19 , when comparing with the Union-only value. This effect can be seen in the reduction in the width of the contours along the $w$-axis in Figure 4,

We now address the reduction in statistical uncertainty on $1+w$ due to adding new nearby SN Ia. We compare this with a $\sqrt{N}$ approximation of the expected reduction in statistical uncertainty. In comparing K08 with WV07, the statistical uncertainty on $1+w$ is reduced from 0.09 in WV07 to 0.079 (averaging the two error bars) in K08 when the same $\mathrm{SN}+\mathrm{BAO}$ fits are compared. This reduces the 0.09 uncertainty by a factor of 1.14 . K08 attribute half the improvement to their eight new SN. This would be a factor of 1.07. K08 say that if, instead of having zero systematic or intrinsic uncertainty, their eight SN Ia had an additional uncertainty of roughly 0.1 mag that the statistical uncertainties on $1+w$ would increase by roughly $10 \%$, going from 0.079 to 0.087 . This would only be a factor of 1.04 decrease over WV07. The Constitution sample statistical uncertainty on $1+w$ would then be smaller than K08 by a factor of 1.3.

Linder (2006) shows that having no nearby SN Ia increases the uncertainty on constant $1+w$ by a factor of two (and that a low-systematic-uncertainty sample of 300 nearby objects is large enough to provide most of the possible nearby statistical leverage). In order to get a rough idea of how much the addition of the CfA3 sample should reduce the statistical uncertainty (without any thought for systematic uncertaintites), it is reasonable to assume that the nearby and faraway samples each contribute half of the statistical leverage. Comparing the Constitution set to the Union set, a simplistic $\sqrt{N}$ analysis would give $\sqrt{147 / 57}=1.6$ for the nearby contribution and 1.0 for high-redshift sample since no new objects are added. Averaging the two gives 1.3. This is higher than the factor of 1.19 from the comparison of the main $\mathrm{SN}+\mathrm{BAO}$ value of $\mathrm{K} 08$ but equivalent to the value that is obtained in the alternative K08 scenario of adding $\sim 0.1$ mag. This suggest that adding the CfA3 sample achieves much of the expected statistical improvement. However, we estimate a systematic uncertainty on $1+w$ of 0.11 in $\S 4.3$. This is roughly $65 \%$ higher than our statistical uncertainty of $\sim 0.067$ and suggests that the addition of the CfA3 sample has now clearly placed SN Ia cosmology in the realm where systematic uncertainties are the most dominant.

The standard deviation of the Hubble residuals is 0.186 mag for the Union (OLD) sample, $0.164 \mathrm{mag}$ for the CfA3 sample and 0.173 mag for the Constitution (OLD+CfA3) sample. The difference in the weighted means of the Hubble residuals of the two nearby samples, OLD minus CfA3, is $-0.017 \pm 0.027 \mathrm{mag}$, showing that the two samples agree within the expected uncertainty. On average, the SALT-based intrinsic luminosities are slightly brighter for the OLD sample than for the CfA3 sample. The CMB redshift, distance moduli and other useful information for the Constitution sample can be found in Table 1 
and can be used by interested parties as an update to the Union compilation for fitting cosmological models.

\subsection{Three Other Fitters}

We summarize the number of objects that passed our minimal cuts in the other three fitters here. The minimal cuts leave samples that have well-fit light curves so that we can explore for any systematic trends in the Hubble residuals that would require further cuts. The minimal-cuts values of $1+w$ for the three samples (OLD, CfA3 and OLD+CfA3) and four fitters are listed in the second portion of Table 5. The results of the best-cuts samples, described in $\S 3.11$, are in the top portion and values of $1+w$ with other cuts are listed in the lower portions of the table. The first column shows $1+w$ for the OLD sample for each of the four fitters, with the mean and standard deviation of these values below. It should be noted that this standard deviation is of the values of $1+w$ from the different fitters, to show how well they agree, and should not be interpreted as a statistical uncertainty on $1+w$. The second column shows the CfA3 results while the third column contains the CfA3+OLD results. The fourth column shows the difference in $1+w$ between the OLD and the CfA3 results for each fitter.

With SALT2, 171 High-z, 65 OLD and 115 CfA3 SN Ia pass the minimal cuts. The CfA3 sample is 1.77 times larger than the OLD sample, after these cuts. In the MLCS31 case, 72 OLD, 129 CfA3 and 165 High-z SN Ia make it through the minimal cuts and the CfA3 sample is 1.79 times larger than the OLD sample. The higher number of nearby SN Ia, as compared to the SALT2 case, is due mostly to including the 1991bg-like SN. The MLCS31 OLD value compares well with the value of $1+w=-0.069_{-0.093}^{+0.091}$ from WV07 who use an older version of MLCS2k2 on the OLD, SNLS, and ESSENCE light curves (no Higher-z data) and have more stringent cuts at $\mathrm{z}=0.015$ and $t_{1 s t}=+4$ days. In the MLCS17 case, 70 OLD, 133 CfA3 and 169 High-z SN Ia survive the cuts. The CfA3 sample is 1.9 times larger than the OLD SN.

\section{3. $\Delta$ and $A_{V}$ Versus Redshift}

We show the distribution of the MLCS2k2 light-curve shape parameters, $\Delta$ and $A_{V}$, versus redshift in Figures 5 and 6 . The CfA3 sample does extend the nearby sample with $A_{V}>0.5$ (and that pass the minimal cuts) out to $z=0.035$. Both highly-reddened and lowluminosity (high- $\Delta$ ) SN Ia are found increasingly at lower redshifts, mostly due to the CfA3 
limiting peak magnitude of $\sim 18.5$ mag. The different SN Ia populations being sampled at different redshifts makes it important to ensure that the light-curve/distance fitters works well across the range chosen for cosmological calculations so as to not introduce a bias.

\subsection{Four-Fitter Comparison of Light-Curve Shape and Reddening}

Next, we want to compare the fitters' output parameters to see that they are reasonably consistent with each other. Figure 7 compares the four fitters' light-curve shape parameters with each other. $\Delta$ (MLCS17) agrees very well with $\Delta$ (MLCS31), as expected, and $s$ and $x_{1}$, from SALT and SALT2, are linearly related with a few outliers. $s$ and $x_{1}$ are nonlinearly related to $\Delta$, the most noticeable feature being that the brightest SN Ia (negative $\Delta$ ) have a wide range of light curve stretch, $s$ or $x_{1}$ (although still at the slow end). At face value, this means that the bright (negative- $\Delta$ ) SN Ia have a wider range of light curve shape than the moderate decliners.

In Figure 8, the MLCS2k2 host-galaxy color excess, $E(B-V)=A_{V} / R_{V}$, from MLCS17 and MLCS31, and the color parameter, $c$ (a combination of host reddening and intrinsic SN Ia redness), from SALT and SALT2 are compared. The upper-left panel shows mostly good agreement. The slope is slightly less than one, meaning that MLCS17 with $R_{V}=1.7$ has a slightly larger best-fit value for $E(B-V)$ than MLCS31 with $R_{V}=3.1$. However, $A_{V}$ will usually be larger for $R_{V}=3.1$. The lower-right panel shows good agreement between $c$ in SALT and SALT2.

In the upper-right and lower-left panels, $c$ should correlate well with $E(B-V)$ in unreddened cases. This is evident in the roughly diagonal lower bound on the locus of points in these two panels and the roughly diagonal lower locus of points in general. The points that extend significantly above this lower locus are mainly SN Ia with higher intrinsic redness that is part of $c$ but not $E(B-V)$. Points that have $\Delta \geq 0.7$-intrinsically redder SN Ia-are shown in red.

Finally, in Figure 9, we plot the difference between the fitters' distance moduli versus redshift. In order to put them on the same scale, the value of $\mathrm{H}_{\mathrm{o}}$ was calculated for the four fitters' samples with the minimal cuts and a correction was added to make them all consistent with $\mathrm{H}_{\mathrm{o}}=65 \mathrm{kms}^{-1}$ and $M_{V}=-19.504 \mathrm{mag}$ for MLCS2k2, $M_{B}=-19.46 \mathrm{mag}$ for SALT and $M_{B}=-19.44$ for SALT2. The actual additive correction is sensitive to the exact subsample used. The upper-left panel shows significant disagreement below $z=0.04$, where the highly-reddened MLCS31 points (shown in red) have significantly shorter distances. This is most likely due to $R_{V}=3.1$ being too high and is discussed further in $\S 3.8$. The low- 
$A_{V}$ points do show excellent agreement though. The slight average offset from zero of the slightly-reddened points is mainly due to the whole MLCS31 sample being adjusted to be consistent with $\mathrm{H}_{\mathrm{o}}=65 \mathrm{kms}^{-1}$, as opposed to only the lowly-reddened portion.

The lower-right panel shows excellent agreement between SALT and SALT2 while the upper-right and lower-left panels show good agreement across redshift, especially when the green points are ignored. These points are in a range $(0.7 \leq \Delta \leq 1.2)$, where MLCS2k2 seems to produce distances that are too short (see H09 and §3.7). This will be discussed further below.

Overall, the four fitters produce light-curve shape and reddening/color parameters that agree well with each other. This is an important result because it shows that they are all similarly characterizing the SN Ia light curves but that determining the distance modulus is an area where different methods can introduce systematic offsets. It is thus a good sign that the distance moduli do not exhibit any trends versus redshift when the areas of low reliability are removed.

\subsection{Twins}

As another test of the light-curve and distance fitting, SN Ia in the same host galaxy"twins"-should produce the same distances. This is also a test of the underlying photometry. One example where this has been discussed in the literature is NGC 1316, the host of SN 1980N and SN 1981D. Hamuy et al. (1991) find that the peak apparent magnitudes agree well but mention that $1981 \mathrm{D}$ has significant host contamination. This makes the photometry of 1981D less reliable. Krisciunas et al. (2000) find that MLCS optical light-curve fits of 1980N and 1981D give almost identical values of $\Delta$, implying similar absolute magnitudes, but due to the estimated host reddening the distance of $1981 \mathrm{D}$ is smaller by about 0.4 mag than that of $1980 \mathrm{~N}$. They find no evidence for host reddening from $V J H K$ analysis but do from the $B V$ data. Since the peak $V$ magnitudes agree well, they conclude that the $B$-band photometry of SN 1981D may have a hidden systematic error. This could well come from the host contamination. A recent addition to this conflicted family-once twins, now triplets-is SN 2006dd (Monard 2006). Hopefully it will settle the score.

On a brighter note, SN 1999cp (Krisciunas et al. 2000) and SN 2002cr occured in the same galaxy, NGC 5468. Optical photometry of SN 2002cr was obtained as part of the CfA3 sample. We fit both SN with all four fitters and the respective agreement in MLCS31, MLCS17, SALT and SALT2 distance moduli, $\mu_{2002 c r}-\mu_{1999 c p}$, are: $0.006 \pm 0.138,0.017 \pm 0.137$, $0.027 \pm 0.195$, and $0.077 \pm 0.233 \mathrm{mag}$. The uncertainties include both the uncertainty from the 
light-curve/distance fit and the additional "intrinsic" uncertainty for each fitter mentioned in $\S 2$.

It should be emphasized that the photometry was aquired and reduced by separate groups and instruments. The excellent agreement in MLCS17, MLCS31 and SALT, and the adequate agreement in SALT2 imply that the photometry from the two groups is accurate and the fitters are working well.

\subsection{Comparing the Results from the Minimal-Cut Samples}

Table 6] shows the standard deviation and the weighted mean of the Hubble residuals of each fitter for each SN Ia sample. We want to know if the two nearby samples are consistent internally and with each other. The similarity in the standard deviations across all four fitters and in the OLD, CfA3 and OLD+CfA3 samples suggests both. With all four fitters, the OLD sample has a more negative mean of its Hubble residuals than the CfA3 sample by about 0.03 mag when all four fitters' values are averaged. This means that the OLD sample is being interpreted as intrinsically brighter than the CfA3 sample. Whether this is due to photometry errors or intrinsic differences in the SN Ia is hard to say. SALT and MLCS17 give differences in mean residuals between the two samples that are within the uncertainty while SALT2 and MLCS31 are slightly outside the quoted uncertainty. The reality of the situation is probably a combination of both photometric offsets and intrinsic SN Ia differences. We examine this further in $\S 3.12$ by calculating the mean residuals for the six or seven main sources of the nearby sample. We find a scatter of $\sim 0.03 \mathrm{mag}$ amongst these subsamples. We conclude that this level of offset between nearby samples is representative of the current state of nearby photometry and light-curve fitting. Since the difference in the mean residuals of the CfA3 and OLD samples, across all four fitters, is roughly at the $1 \sigma$ level it is not too worrisome. Whatever the reason for the difference in the mean residuals, it is consistent with the OLD sample implying larger $\Omega_{\Lambda}$ (with $1+w=0$ ) and lesser $1+w$ than the CfA3 sample. At high redshift, SALT and SALT2 have large standard deviations of their Hubble residuals (see $\S 3.8$ for one possible cause of this) while MLCS17 and MLCS31 have values that are roughly the same as at low redshift. Using the lower wavelength range of $2900 \AA$ should reduce the scatter of the SALT2 high-redshift objects but we do not explore that in this work.

We now turn to looking for trends in Hubble residuals versus light-curve fitter parameters. In $\S 3.7$, we focus on the light-curve-shape parameter and look at the effects on $1+w$ of removing $\Delta>0.7$. In $\S 3.8$, we focus on the color/extinction parameters and look at how only including $-0.1 \leq c \leq 0.2$ for SALT $/ 2$ and $A_{V}<0.5$ for MLCS31 and MLCS17 affect 
$1+w$. In the end, we adopt all of these as the "best" cuts and recalcute $1+w$ in $\S 3.11$.

\subsection{Residuals versus Light Curve Shape}

We use the definition of Hubble residual, $\mathrm{HR} \equiv \mu_{\mathrm{SN}}-\mu_{\mathrm{z}}$, so that a positive residual means that the $\mathrm{SN}$-derived distance is greater than the redshift-derived distance with a given set of cosmological parameters. A positive residual can also be interpreted as the SN being fainter than expected for the reference cosmology, and vice versa for a negative residual. Figure 10 shows the Hubble residuals versus the light curve shape parameters, $s$ and $x_{1}$, for SALT and SALT2, respectively. No significant trends exist in any of the subsamples but it should be noted that there are more broad-shaped SN Ia (large $s$ and $x_{1}$ ) at high redshift and that for SALT2 these have mostly positive residuals, meaning that they are being interpreted as intrinsically fainter than predicted by the best-fit cosmology. Some of the largest outliers in the high-redshift SALT and SALT2 samples are due to a trend in residuals versus color parameter, $c$. Cuts on $c$ that will remove many of these outliers will be explored in $§ 3.8$.

Figure 11 displays the Hubble residuals for MLCS31 and MLCS17 versus $\Delta$. With both fitters, and in both the OLD and the CfA3 samples, there is a peculiar region between $\Delta=0.7$ and $\Delta=1.2$ where the residuals are mostly negative. At high redshift there are only two objects at the edge of this range (due to selection effects) but they also have negative residuals. This "dip" in the residuals is most likely due to a sharp change in the intrinsic absolute magnitude of $1991 \mathrm{bg}$-like SN Ia compared to their neighboring non-1991bg-like SN Ia with slightly lower values of $\Delta$. H09 find that, at peak, the $1991 \mathrm{bg}$-like SN Ia are about 0.5 to 1.0 mag fainter in $B$ and 0.3 to $0.5 \mathrm{mag}$ fainter in $V$ than the non-1991bg-like SN Ia that are nearest to them in $\Delta$. In the nearby samples, the residuals beyond $\Delta=1.2$ are fairly well centered on zero, with a slight positive offset in the CfA3 sample. The inclusion of the 1991bg-like SN Ia in the MLCS2k2 training set gives rise to the positive coefficient for the quadratic term in $\Delta$. The MLCS2k2 model light curves are accurately calibrated for the range of $\Delta$ that the 1991bg-like SN Ia occupy but this comes at the expense of the model light curves being too faint in the region $0.7<\Delta<1.2$. This, in turn, produces negative residuals in this region. MLCS2k2 should be retrained by treating the $1991 \mathrm{bg}$-like objects (either identified spectroscopically or by making a cut above $\Delta \gtrsim 1.2$ ) separately. This will likely improve the performance of MLCS2k2, which already produces smaller scatter than SALT and SALT2 at high redshift.

Another check on this region of $\Delta$ is to compare the differences in the MLCS2k2 and SALT $/ 2$ distances versus $\Delta$. SALT and SALT2 were not trained using $1991 \mathrm{bg}-$ like SN. Figure 12. shows that the MLCS2k2 distances get smaller as compared to SALT/2 as $\Delta$ increases and 
there does seem to be an even steeper drop starting around $\Delta \approx 0.5$. There are no $1991 \mathrm{bg}-$ like SN Ia in the MLCS17-versus-SALT2 plot while in the MLCS17-versus-SALT plot there are two SN Ia with $\Delta>1.2$ because K08 did not exclude 1991bg-like SN Ia and we kept all the K08 SN in our minimal cuts. In spite of the trend in differences in distances versus $\Delta$, it is reassuring that there is no trend versus redshift (see Figure 9). It can be expected that there would be some systematic differences in distances produced by different fitters but we believe that a retraining of MLCS2k2 and SALT2 with larger data sets, avoiding the 1991bg-like SN Ia in MLCS2k2 and better treatment of the $U$ band in SALT2 will bring them into better agreement. Figure 12 also shows that the MLCS17 and MLCS31 distances agree well versus $\Delta$ when the reddened ones are ignored (upper-left panel) and the SALT and SALT2 distances agree well versus $x_{1}$ (lower-right panel).

To avoid the problems of the MLCS2k2 fits with $0.7<\Delta<1.2$, and since the High-z SN only extend out to $\Delta \approx 0.7$, we make a cut on $\Delta>0.7$ and rerun the MLCS31 and MLCS17 cosmology fits. By making this cut we are removing more negative than positive residual objects and so we would expect an increase in $1+w$. For MLCS31, $1+w$ increases by about 0.03 to 0.035 for each of the OLD, CfA3 and OLD+CfA3 subsamples, and a similar effect is seen for MLCS17. The specific results are presented in Table 5. In $§ 4.2$, we will look for any trends in residuals and $\Delta$ versus host galaxy morphology.

As SN Ia samples increase in size it may prove worthwhile to only include objects that are within the light-curve-parameter regions that are common to both high- and low-redshift samples. It is uncertain if our cut on $\Delta$ moves our results on $1+w$ closer to physical reality since we are removing a region of the parameter space that was used in the training. Ideally, the training of the light-curve/distance fitter would be performed over the same range of parameters that is used in the cosmological calculations. The cut at $\Delta=0.7$ will be one part of our best cuts. The other part wil be to only include objects with $-0.1 \leq c \leq 0.2$ and $A_{V} \leq 0.5$, which we discuss next.

\subsection{Residuals versus $c$ and $A_{V}$; Discussion of $\beta$}

Figure 13 shows the difference between distance moduli for the four fitters versus color parameter. The comparisons between MLCS17, SALT and SALT2 all show no trend while it is very clear that the higher value of $R_{V}=3.1$ makes the MLCS31 distances increasingly smaller than the MLCS17 distances with higher host reddening.

In Figure 14, we plot the Hubble residuals versus $c$ for SALT and SALT2, with the minimal cuts (for SALT, these are the Union cuts from K08). Neither of the nearby samples 
show any significant trend but the high-redshift sample has highly positive residuals at the blue end with both SALT and SALT2 and several highly negative residuals at the reddest end with SALT. The High-z SALT sample shows a clear negative slope with increasing color. We explore the effects on $1+w$ by making a sensible cut on $c$.

Most of the OLD SALT SN Ia fall within the range $-0.1 \leq c \leq 0.2$. Cutting all objects outside this range would remove most of the extreme outliers in the SALT High- $z$ sample. We choose this as our cut on $c$ for SALT. The High- $z$ SALT2 sample does not have the same problem at the red end but we make the same cut on $c$ to be consistent. Also, $c \approx 0.2$ corresponds well with this value. If a typical unreddened $s \approx 1$ SN Ia has $c \approx 0$ then this same SN with $c \approx 0.2$ would experience $0.2 \mathrm{mag}$ of host reddening. If we assume that $R_{V} \approx 2.5$, a rough average of the two values in this paper, then $c \approx 0.2$ corresponds to $A_{V} \approx 0.5$ and these cuts on $c$ and $A_{V}$ are fairly consistent.

Without the cuts on $c$, SALT and SALT2 have respective values of $\beta$ of $2.59_{-0.08}^{+0.12}$ and $2.48_{-0.12}^{+0.10}$ for the OLD+CfA3 samples (including High-z) and $2.87_{-0.17}^{+0.17}$ and $2.69_{-0.21}^{+0.22}$ with the cuts on $c$. The corresponding values of $\alpha$ are $1.34_{-0.08}^{+0.08}$ and $1.26_{-0.09}^{+0.09}$ for SALT, before and after the cut on $c$, and $0.104_{-0.018}^{+0.018}$ and $0.109_{-0.021}^{+0.026}$ for SALT2. We also fit after only cutting out the objects bluer than $c=-0.1$ and find $\beta=2.55_{-0.10}^{+0.10}$ for SALT2 $\left(\alpha=0.106_{-0.019}^{+0.019}\right)$. This cut does not remove the redder ones. It is only $0.14 \pm 0.24$ lower than the SALT2 case where the additional cut above $c=0.2$ is also made. This difference in $\beta$ of 0.14 is small but implies that the reddening law for dust is smaller for highly-extinguished SN Ia. When $\beta$ is calculated in SALT for the minimal-cuts High-z and nearby objects separately, they are virtually the same, consistent with the findings in K08. In SALT2 they differ by $0.14 \pm 0.22$, within the $1 \sigma$ uncertainty. This suggests that the single color correction of the nearby and faraway SN Ia is adequate. However, there is a large difference in SALT between $\alpha=1.13_{-0.08}^{+0.09}$ in the low-redshift sample and $\alpha=1.75_{-0.13}^{+0.15}$ in the high-redshift sample: $0.62 \pm 0.16$. In SALT2, $\alpha$ is $0.037_{-0.039}^{+0.029}$ larger in the high-redshift sample than in the nearby. Since the nearby and High-z values of $\beta$ are consistent with each other, but the values of $\alpha$ are not (more in SALT than SALT2), it is interesting that the trend in High-z residuals is seen versus color, $c$, and not stretch, $s$ (see Figures 14 and 10).

If there were no differences in intrinsic SN Ia color then $c$ would just be the host reddening and $\beta$ would be equivalent to $R_{B}=R_{V}+1$. Even though $\beta-1$ is not equivalent to $R_{V}$, it is interesting to see that the values of $\beta-1$ are about 1.55 without the cuts on $c$ and 1.78 with the cuts, both very close to the value of $R_{V}=1.7$ derived to minimize the dependence of the nearby Hubble residuals on $A_{V}$.

In Figure 15, we plot the Hubble residuals versus $A_{V}$ for MLCS31 and MLCS17. All three subsamples (CfA3, OLD and High-z) have mostly negative residuals beyond $A_{V}=0.5$ 
with MLCS31. The slope in residuals versus $A_{V}$ for MLCS31 is -0.34 both with or without the cut on $A_{V}$ while for MLCS17 it is +0.01 (nearly zero) with the cut at $A_{V}=0.5$ and +0.12 without it. Although a slightly higher value in $R_{V}$ would eliminate the trend in residuals versus $A_{V}, R_{V}=1.7$ succeeds in eliminating the trend in residuals versus $A_{V}$ when the cut at $A_{V}=0.5$ is made. $A_{V}=0.5$ is adopted as a cut-off value for our best cuts in $\S 3.11$ and so there is no point in searching for a higher value of $R_{V}$ that would eliminate the trend at greater $A_{V}$. The fact that, without the cut on $A_{V}$, MLCS17 has a slightly positive slope while it was practically zero for the low- $A_{V}$ objects implies that the high-extinction objects are interacting with dust with larger $R_{V}$ than the dust of the low-extinction objects. This is the opposite of what was seen in SALT2 and suggest that neither implication about the dust in the highly-extinguished objects should be taken seriously.

If we assume that highly reddened SN Ia are intrinsically similar to their less-reddened counterparts with similar $\Delta$ then this means that $A_{V}$ (with $R_{V}=3.1$ ) is overestimated, giving rise to smaller MLCS31 distance moduli than those from the best-fit cosmology. This seems more likely than assuming that the highly-reddened SN Ia are intrinsically brighter and that the MLCS31 $A_{V}$ is accurate. In contrast, the MLCS17 Hubble residuals show little trend with respect to $A_{V}$, especially when cut at $A_{V}=0.5$.

Table 5 shows the values of $1+w$ after making the cuts on $c$ and $A_{V}$. SALT, SALT2 and MLCS31 all have greater values of $1+w$ for the OLD + CfA3 samples while MLCS17 has a smaller value of $1+w$, bringing them into better agreement with each other. The standard deviation of $1+w$ from the four fitters drops from 0.051 to 0.025 for the OLD+CfA3 samples, from 0.051 to 0.046 for the CfA3 sample and from 0.075 to 0.059 for the OLD sample, and the average difference in $1+w$ between the OLD and the CfA3 samples drops from -0.068 to -0.060 , suggesting an improvement in our calculation of $1+w$. While the four fitters agree better now for a given sample, and the mean value of $1+w$ from each sample agrees slightly better, the CfA3 and OLD samples do not improve in agreement for any single fitter.

Until the intrinsic color variation of SN Ia and the host dust properties are better understood, and distance fitters are better able to incorporate this information, it is safer to cut out the overly blue and red objects in SALT and SALT2 and the moderate to high extinction objects in MLCS2k2. The improved agreement in $1+w$ between the four fitters and the relative constancy in the uncertainties on $1+w$ suggest that the cuts are worth making. The cuts on $c$ and $A_{V}$ will be combined with the cut on $\Delta$ to form our best cuts. 


\subsection{No $U$ band}

Jha et al. (2006) present the first large body of nearby $U$-band light curves. H09, as part of the CfA3 sample, present an even larger body. Ellis et al. (2008) compile a large body of rest-frame UV spectra of SN Ia at $z \approx 0.5$ and Foley et al. (2008) do something similar. The $U$ band is the most poorly-constrained optical band for SN Ia. It is also poorly fit by SALT2, where the model spectra in the rest-frame $U$ region are highly influenced by the high- $z U$ band photometric calibration, which might be discrepant from that at low $z$. It is worth briefly examining the effects of excluding the rest-frame $U$ band from the light curve and cosmology fits. We refit the nearby SN Ia samples with MLCS31 and SALT2, excluding the $U$ photometry. Many of the high-redshift SN Ia have strong contributions from rest-frame $U$. To check if this matters we fit the cosmology for the no- $U$ nearby samples plus the ESSENCE sample, since ESSENCE is mostly composed of rest-frame $B$ and $V$ data. There is virtually no difference between the values of $1+w$ found using just the ESSENCE sample as the High-z sample and using the complete High-z sample (the uncertainty is larger though when using only ESSENCE as the High-z sample, due to fewer objects) so we choose to focus on the cosmology fits of the no- $U$ nearby samples combined with the complete High-z samples. All values of $1+w$ increase by about 0.03 for the three MLCS31 samples. The OLD SALT2 sample has its value of $1+w$ increase by about 0.02 , the CfA3 SALT2 $1+w$ is virtually unchanged and the OLD+CfA3 SALT2 $1+w$ increases by an insignificant 0.005 . SALT2 was designed partially to use the rest-frame $U$-band data. The MLCS17 CfA3 value of $1+w$ is virtually unchanged as well. See the exact values in Table 5. The change in the MLCS31 values may be due to the importance that the $U$ band plays in determining the color excess (and subsequent extinction, $A_{V}$ ), especially for SN Ia in dusty host environments. Because of the negligble effect on MLCS17 and SALT2 and the 0.03-level effect on MLCS31 we do not explore this further beyond combining the no- $U$ cuts with cuts on $A_{V}$ and $\Delta$ in $\S 3.10$.

\subsection{Combining Multiple Cuts}

We briefly explore making a cut at $A_{V}=0.5$ on the no- $U$ MLCS31 sample (see Table 5). The results are quite similar to the cut at $A_{V}=0.5$ on the MLCS31 sample (with $U$ ). This is consistent with the possibility that the $U$ band has a strong impact on the extinction estimate for the more highly-reddened objects. If we remove these objects with the cut at $A_{V}=0.5$ then it is not so important whether the $U$ band is kept or not in the nearby samples. The MLCS17 CfA3 sample, which we expect to be not as sensitive to reddening effects, has its value of $1+w$ decrease by 0.009 , compared to the no- $U$ case.

We also cut out SN Ia with $\Delta \geq 0.7$ and $A_{V}>0.5$ for the no- $U$-band MLCS31 samples 
(see Table 5). Just as we saw an increase in $1+w$ by about 0.03 to 0.035 in making only a $\Delta>0.7$ cut on the MLCS31 sample, we also see a similar increase in $1+w$ compared to the no- $U$ plus $A_{V} \leq 0.5$ sample from the previous paragraph while the MLCS17 CfA3 sample increases by 0.05 relative to the value in the previous paragraph. This is to be expected since the $\Delta>0.7$ cut removes more negative than positive nearby residuals while leaving the High-z sample virtually unchanged.

\subsection{Best Cuts Adopted}

Finally, for MLCS17 and MLCS31, we combine the cuts of the two areas of most-obvious difficulties, namely, cutting above $A_{V}=0.5$ and above $\Delta=0.7$. We adopt these cuts (on top of the minimal cuts) as our best cuts for the two MLCS2k2 fitters.

Both of these cuts remove more negative-residual objects than positive and this causes $1+w$ to rise to $0.074_{-0.065}^{+0.064}$ for MLCS31 and $0.118_{-0.065}^{+0.063}$ for MLCS17, both with the OLD+CfA3 sample (see Table 5). The statistical uncertainties on $1+w$ are lower than for MLCS31 and MLCS17 with the minimal cuts, in spite of fewer objects.

For SALT and SALT2, the best cut is to include objects with $-0.1 \leq c \leq 0.2$. The OLD + CfA 3 values of $1+w$ for SALT and SALT2 are $0.026_{-0.071}^{+0.069}$ and $0.026_{-0.082}^{+0.078}$, respectively, in excellent agreement with each other.

Amongst the four fitters, SALT and MLCS17 give the lowest differences in weightedmean residuals and have the best agreement between the CfA3 and OLD values of $1+w$. With the best cuts, MLCS17 and MLCS31 have the smallest statistical uncertainties on $1+w$. SALT2 and MLCS31 produce the highest difference in weighted-mean residuals between the OLD and the CfA3 samples and they produce the greatest differences between these two samples' values for $1+w$.

In all three samples, the MLCS17 values of $\Omega_{\Lambda}(1+w=0)$ are the lowest and the MLCS17 values of $1+w$ are the largest. With its lower value of $R_{V}$, MLCS17 attributes less host extinction to each SN Ia. This produces a larger distance compared to MLCS31 for the more-reddened nearby SN Ia, and from that, a larger value of $1+w$. Although there are some highly-reddened SN Ia in the High-z sample, most of them are below $z \approx 0.35$ and do not affect $1+w$ as much as if they were at $z \approx 0.5$. The High-z sample is relatively unreddened in the redshift region that has most influence on $1+w$.

It is also important to look at the standard deviations of $1+w$ for the four fitters in the OLD and the CfA3 samples (Table 51). They are roughly the same: 0.066 and 0.064, 
respectively. The fitters were all trained on many objects from the OLD sample and none from the CfA3 sample, yet the CfA3 sample gives a similar standard deviation in $1+w$. This suggests that the CfA3 sample is internally consistent. Also, as seen in Table 6 and discussed in $§ 3.6$, the scatter in the residuals of the OLD and the CfA3 samples is similar.

Repeating the simplistic $\sqrt{N}$ analysis of $\S 3.1$, of attributing half the statistical leverage to the faraway sample and half to the nearby sample, we expect a decrease in the statistical uncertainty of $1+w$ to be roughly 1.3 in the four fitters. We find a decrease of roughly 1.2 1.3. It is likely that we are beginning to run up against the effects of systematic differences in different light curve samples and in different light-curve/distance fitting assumptions and methods.

The fact that our best cuts on MLCS31 and MLCS17 give values of $1+w$ about 0.05 and 0.09 higher than the SALT/2 values is somewhat troubling. In the case of MLCS2k2, although these seem like the correct regions to cut out, it may introduce a bias to the remaining SN, since it was trained on the larger range of values. We expect that if the CfA3 sample is added to the training sets, the 1991bg-like SN Ia are treated separately (in MLCS2k2), improved treatement of the $U$ band is achieved, and the correct reddening treatment is discovered and used, that the retrained fitters (SALT/2 and MLCS2k2) will then produce values of $1+w$ that agree well with each other.

\subsection{Mean Hubble Residuals of Six Different Nearby Samples}

We have already looked at how the weighted mean of the Hubble residuals of the OLD and CfA3 nearby samples agrees in the four fitters. In MLCS17 and SALT we found better than $1 \sigma$ agreement while in MLCS31 and SALT2 it was slightly larger than $1 \sigma$. We now want to compare the main sources of nearby data more closely to see if subsamples of the OLD and CfA3 samples are causing the slight discrepancy. We break up the OLD sample into the Calan-Tololo (CT) (Hamuy et al. 1996a), CfA1 (Riess et al. 1999), CfA2 (Jha et al. 2006) and Other subsamples (and K08 in the case of SALT where the eight SN Ia that K08 present are included in the Union and Constitution samples). We also break up CfA3 into CfA3(4Sh) and CfA3(Kep) for the two cameras (4Shooter and Keplercam, see H09 for details) that observed the vast majority of the CfA3 sample.

For SALT and SALT2, since there are no obvious trends in residuals versus stretch or color at low redshift (it was the trend versus color at high redshift that necessitated the cut on $c$ ), and we are only looking at nearby objects for this comparison, we use the minimal-cuts nearby sample. For MLCS17 and MLCS31, we look at both the minimal-cuts sample and 
the best-cuts $\left(A_{V}\right.$ and $\left.\Delta\right)$ sample.

For each subsample we calculate the weighted mean of the Hubble residuals and the uncertainty. In the best-cuts cases of MLCS17 and MLCS31, we are still using the residuals from the minimal-cuts cosmological fits and so we subtract off the weighted mean of the entire best-cuts sample and recalculate the difference between the OLD and the CfA3 mean residuals. The standard deviation of the subsamples' weighted means is calculated both with and without the most-discrepant OLD subsample. We also remove the most discrepant OLD subsample and recalculate the modified OLD*_CfA3 differences. The CfA3(Kep) sample has the largest influence on the mean of the whole sample due to its size. The results are displayed in Table 7. We reiterate that the CfA3 sample played no role in training the light-curve fitters and so the good agreement between the two CfA3 subsamples and most of the others is a good sign that the training samples are representative of the nearby SN Ia population and working well for the most part.

In both MLCS17 and MLCS31, for both the minimal and the best cuts, the CfA1 sample is the most discrepant. In MLCS17, the other five subsamples all agree to better than $1 \sigma$. In MLCS31, the best cuts actually make the two CfA3 subsamples disagree more but this may be due to different distributions in $A_{V}$ where the slope in residuals versus $A_{V}$ of -0.34 is present.

SALT2 has the largest discrepancies between all the subsamples, with CT being the most discrepant from the mean of the whole sample. SALT has seven subsamples and Other is the most discrepant but it only has five objects.

Some of the differences between subsamples is likely due to systematic offsets in photometry but a good portion is also due to the selection effects that different cuts have on which objects remain. The agreement between OLD and CfA3 mean residuals improves with the best cuts for MLCS17 and stays about the same for MLCS31. When the most-discrepant subsample is removed from the OLD sample, the resulting OLD*_CfA3 differences are all at the $1 \sigma$ level or less. As a crude way of measuring the scatter in the subsamples in the different fitters, we ignore the most discrepant subsample and take the standard deviation (StdDev*) of the weighted means. MLCS17 has 0.02, MLCS31 and SALT have 0.03 and SALT2 has 0.06. If we take $0.03 \mathrm{mag}$ as representative, then that is about how good any subsample's weighted mean of its Hubble residuals can be expected to agree with another's. Hopefully, the inclusion of several subsamples reduces the offsets from the true value of the individual subsamples.

As we have seen in this work, in comparing the OLD and the CfA3 samples, the difference in $1+w$ is about twice as large as the difference in the weighted mean of the residuals. 
Going forward, to not only measure static $1+w$ better but to measure for time variation as well, SN Ia photometry needs to be improved by lowering systematic errors in calibration and understanding passbands better. Improved signal-to-noise measurements will also be helpful. The light curve and distance fitting needs to be more robust, including how it deals with host-galaxy reddening, so that the distances do not have systematic errors.

\section{Looking for Physical Systematics}

\subsection{Hubble Bubble}

We address the issue of whether we live inside a Hubble bubble, possibly due to a local void, where space is expanding faster (or slower) within a given region than outside. Zehavi et al. (1998) find an apparent bubble. J07 confirms its existence, finding the inner expansion rate to be $6.5 \pm 1.8 \%$ higher than the outer rate at the partition redshift of $z \approx 0.025$. Conley et al. (2007) shows that MLCS2k2, with $R_{V}=3.1$, and SALT, SALT2, and SiFTO, with $\beta=4.1$, all show evidence of a Hubble Bubble but that SALT, SALT2 and SiFTO do not produce significant bubbles when the best-fit value of $\beta \approx 2$ is used.

We follow the same approach as J07 and Zehavi et al. (1998), converting our SN Ia lightcurve-fitter-derived distance modulus, $\mu_{L C}$, into a luminosity distance, $d_{L C}$ and multiplying by $H_{o}$ to get the light-curve-based expansion velocity. We also take the luminosity distance predicted by the best fit cosmology, $d_{\text {cosm }}(z)$ and multiply by $H_{o}$ to get the best-fit-cosmology velocity. We subtract the two to get the host galaxy peculiar velocity, $u=H_{o} d_{\text {cosm }}(z)-H_{o} d_{l c}$. The deviation from the Hubble law is equivalent to the fractional peculiar velocity and can be expressed as $\delta H / H=u /\left(H_{o} d_{l c}\right)$. This is displayed for the SALT results in the top panels of Figure 16. We partition the nearby samples in increments of $\Delta z=0.001$, starting at $z=0.015$ for MLCS31, MLCS17, and SALT2, and $z=0.018$ for SALT and ending at $z=0.06$ for all fitters for the OLD and OLD+CfA3 samples and at $z=0.05$ for the CfA3 sample. These ranges on the redshift partition ensure adequate numbers of SN Ia both above and below each partition. For each partition, we calculate the best-fit Hubble

constants $H_{\text {inner }}$ and $H_{\text {outer }}$ and their uncertainties as in Zehavi et al. (1998). We calculate the void amplitude, $\delta_{H}=\left(H_{\text {inner }}-H_{\text {outer }}\right) / H_{\text {outer }}$, and its uncertainty. These are displayed in the middle panels of Figure 16. Finally, in the bottom panels, we show the void significance, $\delta_{H} / \sigma$.

For the minimal-cuts samples, we look for the largest absolute value of the void significance to identify the most likely redshift for a Hubble Bubble. Table 8 shows the results from the various fitters and samples. The minimal-cuts samples are used for all four fitters as well 
as the $A_{V} \leq 0.5$ samples for MLCS31 and MLCS17. We find a Hubble bubble at $z \sim 0.025$ in the OLD samples with both MLCS31 and SALT, with significances of 3.0 and 2.0, respectively, while MLCS17 and SALT2 do not yield significant Hubble bubbles in the OLD sample. The SALT and MLCS17 CfA3 samples show negative Hubble Bubbles at $z \approx 0.035$ with absolute-value void significances greater than 3.0, and the SALT2 CfA3 sample shows one there with signficance of 1.6. The MLCS31 CfA3 sample has its most significant void at $z=0.04$. The difference in void location between the CfA3 and OLD samples illustrates the sample-dependent nature of Hubble bubble calculations.

Adding the samples should amplify any real voids. However, we see the opposite. Each of the most-significant voids in the OLD and CfA3 samples becomes weaker and, in some cases, replaced by a different most-significant-void redshift. The SALT and SALT2 voids have a significance on the order of 1 . MLCS17 has a $2.75 \sigma$ void at $z=0.034$ with amplitude -0.020 while MLCS31 has a $5.56 \sigma$ void at $z=0.028$ with amplitude 0.029 .

By adding the CfA3 sample we have shown that the MLCS31 Hubble bubble is smaller and at a slightly different redshift than was seen in Jha and Zehavi. The negative bubble in the case of MLCS17 shows that the existence of a Hubble bubble in any data set may be influenced by the reddening law, $R_{V}$, used. Whereas in Conley they show that by using a larger $\beta$ they can find positive Hubble bubbles in SALT, SALT2, and SiFTO, we show that by using a smaller value of $R_{V}$ in MLCS2k2 that the positive Hubble bubble is removed and even becomes negative.

As was discussed above, if the MLCS31 values of $A_{V}$ are indeed being overestimated then the light-curve-derived distance modulus will be underestimated. The distance modulus calculated from the redshift and the best-fit cosmology will be larger than the light-curvederived one, giving rise to a positive peculiar velocity. This seems to be why MLCS31 is finding a Hubble bubble.

We return to the $A_{V}$-versus-redshift plot for the three MLCS31 samples in Figure 6, It is interesting to note that the $A_{V}>0.5 \mathrm{SN}$ Ia are at $z<0.035$ for the CfA3 sample and at $z<0.025$ for the OLD sample, very close to the values at which the most significant Hubble bubbles are seen. We redo the MLCS31 and MLCS17 Hubble bubble calculations, removing all objects with $A_{V}>0.5$. We show the results for MLCS31 in Figure 17. The most-significant void amplitudes of the OLD+CfA3 samples are now just slightly greater than one, similar to those of SALT and SALT2. We conclude that any Hubble bubble with redshift partition in the range $0.015 \leq z \leq 0.060$ is likely to be less than $2 \%$ and could be positive or negative. Our analysis is consistent with no Hubble bubble. This has important implication for SN cosmology since nearby SN Ia down to $z \approx 0.01$ can be safely included. We make an estimate of the systematic uncertainty due to a potential Hubble bubble in $§ 4.3$. 


\subsection{Host Galaxy Morphology and Projected Galactocentric Distance}

In Figures 18 and 19, we plot the Hubble residuals, color or host-reddening, and lightcurve shape parameter versus the host galaxy morphology and projected galactocentric distance (PGCD) for SALT2 and MLCS17, using the best cuts for the Hubble residuals panels. The SALT2 plot is very similar to SALT and the MLCS17 plot is very similar to MLCS31 so we only show these two. It should be reiterated that our samples are not complete, especially for the nearby samples where field galaxies tend to be searched less than clusters. We are only reporting the SN Ia that were discovered, have good light curves and have morphological typing. Morphological type is drawn from NED1. About $70-75 \%$ of the nearby SN Ia host galaxies have morphological typing listed in NED. For the color/reddening and light-curve-shape plots we want to focus on the range of nearby SN Ia properties and not just those in the Hubble flow so we do not impose any cut on redshift. We are not trying to ensure high-precision distances in these non-residuals panels so we also relax the time of first observation to +20 days and allow for a slightly higher $\chi_{\nu}^{2}$ (roughly $30 \%$ higher than in the minimal cuts). These are still stringent enough to ensure that the light-curve parameters are useful. For example, J07 use +20 days as their cut on $t_{1 s t}$. We remind the reader that PGCD is a lower limit on the actual distance from the host center. Several interesting properties are seen.

\subsubsection{Light-Curve Shape}

First, we focus on the light-curve shape plots in the lower panels of Figures 18 and 19.

There are several negative- $\Delta$ (broad light-curve shape) SN Ia in elliptical and S0 hosts. Due to the fact that stretch $\left(s\right.$ and $\left.x_{1}\right)$ and $\Delta$ are not linearly correlated, there is a difference in their respective distributions versus morphology. The largest-stretch SN Ia are clearly found in the Sb-Sc hosts and not in the Sd/Irr or E/S0 hosts while the distribution of $\Delta$ is much flatter across host morphology. Close examination does show a gentle maximum of negative- $\Delta$ in the Sb-Sc galaxies but with negative- $\Delta$ objects in E/S0 and Sd/Irr hosts as well. An example of this is the two most-negative- $\Delta$ SN Ia in the E hosts: SN 2002dj and 2008bf. SN 2002dj has $\Delta=-0.20, s=0.93$ (but with a poorer SALT fit) and $x_{1}=-0.114$. SN 2008bf has $\Delta=-0.18, s=1.04$ and $x_{1}=0.17$, with the highest $s$ and $x_{1}$ for SN Ia within E hosts. Both of these are at the high end of the range of $s$ and $x_{1}$ within E hosts but they do not seem particularly high when compared to the highest values seen in the Sb

\footnotetext{
${ }^{1}$ http://nedwww.ipac.caltech.edu/
} 
hosts. Nonetheless, they do extend the range of light curve shapes seen in E hosts.

Additionally, the CfA3 sample adds many slow decliners. This is very important since high redshift searches are often biased towards finding these broad and bright SN Ia. Many of these are found at high PGCD, something that was not seen often in previous nearby samples. There is an absence of very-fast decliners $\left(x_{1}<-3 ; \Delta>0.8\right)$ in Sbc-Irr galaxies

but the CfA3 sample does extend the range in these galaxy types to include faster decliners than typically seen in the OLD sample. As was seen in the OLD sample, most of the CfA3 high- $\Delta$ (low-luminosity) SN Ia are found closer to their host centers.

\subsubsection{Reddening and Extinction}

Looking at the plots of $A_{V}$ and $c$ in the middle panels of Figures 18 and 19 shows the striking feature that the estimated extinction or color is roughly as low in the Scd/Sd/Irr galaxies as it is in the E/S0 hosts while the higher extinction and colors are seen in the middle-type spirals. The MLCS17 plot shows this best but the trend is there with all four fitters. With a larger sample it is also interesting to see that most of the reddened SN Ia occur within 10-12 kpc of the host center.

To get a three-dimensional perspective we plot PGCD versus morphology and colorcode $A_{V}$ and $\Delta$ for MLCS17 in Figure 20, In the top panel, blue means low reddening and red means high reddening and we see that the SN Ia found in the Scd and Sd/Irr hosts are lowly-reddened (mostly blue and green points) and only extend out to $\sim 18 \mathrm{kpc}$. This suggests that although Scd and Sd/Irr galaxies have a lot of gas that can form new stars they do not have a lot of dust that reddens SN Ia. As we move into the Sc and Sb spirals we see reddening start to occur near the galaxy centers and move outwards, perhaps suggesting a change in the amount or type of dust in the inner regions. The projected distance at which SN Ia are found also increases. In the lower panel, blue means bright and red means faint and there does not seem to be much of a trend in the distribution of $\Delta$ along PGCD in the Sb-Irr galaxies but it is very interesting to start to see faint, fast decliners (red) in the central regions of the Sa galaxies, where the star populations are older.

Then, as we move into the S0 and E galaxies in the top panel of Figure 20, we again see a change in the SN Ia reddening. It is not completely gone, as evidenced by the green symbols! But it is once again at levels seen in the Scd/Sd/Irr spirals and irregulars. The distribution of $\Delta$ throughout the $\mathrm{S} 0$ and $\mathrm{E}$ hosts in the lower panel seems fairly uniform. Especially of note is the fast decliners occuring at both small and large PGCD, in agreement with a relatively uniform and old stellar population throughout the E/S0 galaxies. 
We have just narrated the change of both supernova and dust properties as we move from Sd/Irr to E/S0 galaxies and their presumed star populations. Scannapieco \& Bildsten (2005), Mannucci, Della Valle, \& Panagia (2006) and Sullivan et al. (2006) find two populations of SN Ia progenitors, one "prompt", that follows the star-formation rate, and one "tardy", that follows the mass distribution. The fast-declining SN Ia occur in regions of older star populations while the slow-declining SN Ia predominantly occur in regions of intermediate star populations and star formation (Sa-Sc galaxies for SALT and Sb-Sc for SALT2). This is slightly different than the mantra that the brightest, slowest SN Ia are found in the youngest galaxies. Bright SN Ia (negative $\Delta$ ) are certainly found in the Sd/Irr hosts but the slowest decliners (largest stretch) are not. Meanwhile, the "normal" SN Ia occur throughout all morphologies and PGCD. Sullivan et al. (2006) show that at high redshift the high-stretch SN Ia are found in star-forming galaxies while low-stretch SN Ia are found more predominantly in passive hosts and a mix is found in weakly star-forming hosts. Gallagher et al. (2008) find a similar trend, where high star-formation rate galaxies have a flat distribution of of bright and normal SN Ia while moderate star-formation rate galaxies host a wide range of SN Ia and the very-low star-formation rate galaxies do not host the brightest SN Ia but do host faint SN Ia. There is a definite uptrend in stretch from Sd/Irr to $\mathrm{Sb}$ (Figure 18).

It is tempting to speculate that the prompt component is related to the normal decliners of the type found in the $\mathrm{Sd} / \mathrm{Irr}$ hosts while the tardy component is related to the fast decliners. Normal decliners in older hosts may be slightly different beasts from those in young hosts. Whether the slowest decliners can be assigned to either prompt or tardy, or split between the two somehow is unclear. Perhaps there is a third population that is neither prompt nor

tardy and gives rise to the slowest decliners. Or, perhaps there is more of a continuum of progenitors.

\subsubsection{Hubble Residuals and Three Morphology Bins}

We now focus on the Hubble Residual plots in the upper panels of Figures 18 and 19, The SN Ia in the problematic regions of $A_{V}>0.5$ and $0.7 \leq \Delta \leq 1.2$ have been excluded from these plots and the calculations that follow. We notice that the residuals of the SN Ia in ellipticals have more negative residuals with all four fitting methods while the SN Ia in Scd and Sd/Irr hosts have more positive residuals. We divide the SN Ia in these plots into three groups: E-S0, S0a-Sc, and Scd/Sd/Irr. We calculate the standard deviation and the weighted mean of the residuals in each subsample, for each fitter. The results are shown in Table 9. For each fitter's results, we find that SN Ia in E-S0 hosts have negative mean 
residuals beyond the $1 \sigma$ level and that the $\mathrm{SN}$ Ia in Scd/Sd/Irr hosts have positive mean residuals beyond the $1 \sigma$ level, except for the SALT Scd/Sd/Irr subsample that is nearly at the $1 \sigma$ level. The negative residuals in the E-S0 hosts is somewhat similar to the $1 \sigma$ negative mean residual found by $\mathrm{J} 07$ in E hosts. We find the average residual in the Scd/Sd/Irr hosts is $0.091 \pm 0.057 \mathrm{mag}$ while in the $\mathrm{E} / \mathrm{S} 0$ hosts it is $-0.054 \pm 0.041 \mathrm{mag}$, leading to the average residual in the $\mathrm{Scd} / \mathrm{Sd} / \mathrm{Irr}$ hosts being $0.144 \pm 0.070 \mathrm{mag}$ larger than the average $\mathrm{E} / \mathrm{S} 0$ residual, a $2 \sigma$ measurement. This is not an effect of host reddening since neither the E/S0 nor the Scd/Sd/Irr hosts produce highly-reddened SN Ia. It is not the effect of the negative MLCS2k2 residuals in the range $0.7 \leq \Delta \leq 1.2$ since those were removed. We examine some of the light curve fits of the most severe outliers for irregularities but find no good reason to remove them. The residuals in the intermediate group of S0a-Sc spirals are mostly consistent with zero mean residuals. The fact that each fitter finds the same trend is reassuring. These results suggest that SN Ia are different, after light-curve and color/reddening correction, in the E/S0 and Scd/Sd/Irr galaxies. It may be fruitful to train the various light curve fitters separately on the three subsets of host galaxy types.

In Figure 21, in the bottom left panel, we plot the MLCS17 Hubble residuals versus morphology and color code $\Delta$ so that the very faint SN Ia are red $(\Delta>1.2)$, faint ones are orange $(0.7 \leq \Delta<1.2)$, normal and moderately faint ones are green $(-0.1 \leq \Delta<0.7)$, and bright ones are blue $(\Delta<-0.1)$. The most-negative residual SN Ia in the E and S0 hosts are faint (orange), but not very faint (red). We reiterate that the $A_{V}>0.5$ and $0.7 \leq \Delta \leq 1.2$ objects were not included in calculating the mean residuals and scatter in the three morphology bins but we show them in 21 so that their distribution can be seen.

We also find that the Scd/Sd/Irr subsample has the lowest average scatter across the four fitters (0.100 mag) while the E-S0 subsample scatter (0.182 mag) is slightly larger than that of the S0a-Sc subsample (0.166 mag) (see Table 9). At face value this seems to disagree with the findings by Sullivan et al. (2003), where the three partitions of SN Ia we have used have $\sigma=0.16, \sigma=0.20$, and $\sigma=0.27 \mathrm{mag}$, respectively. However, they use the stretch method that attempts no color or reddening correction and many of their objects are at high redshift. We find that two of the arguments that are used to promote the search for SN Ia in elliptical galaxies at high redshift would also apply to the Scd/Sd/Irr hosts: namely, that the extinction is low and the intrinsic dispersion is small. Granted, our study only looks at the low redshift sample so we cannot say if the high redshift SN Ia in Scd/Sd/Irr galaxies have this same low reddening and dispersion but it is worth keeping in mind in designing future SN Ia surveys. Our finding of high dispersion in the early-type hosts, if not just an artifact of the four fitters we use, would weaken but not kill the argument for searching for high-redshift SN Ia in early-type hosts. The reddening would still be low but the scatter in the Hubble residuals may not be as low as expected. 
Finally, we divide the nearby sample into inner and outer subsamples at PGCD $=12 \mathrm{kpc}$, where the distribution of highly reddened objects ends. We find that the scatter is about $0.05 \mathrm{mag}$ less in the outer subsample with three of the four fitters and about the same in the fourth. This may be informative to high-redshift searches in that higher-dispersion and highly-reddened SN Ia can be avoided by not including SN Ia within $\sim 10 \mathrm{kpc}$ of the host center.

\subsection{Systematics}

WV07 present a thorough list of potential sources of systematic error on the measurment of $1+w$, leading to an estimate of $\Delta_{w}$ (syst $)=0.13$. This is larger than what is presented by A06 and K08: $\Delta_{w}($ syst $)=0.054$ and $\Delta_{w}($ syst $) \approx 0.085$, respectively for their $\mathrm{SN}+\mathrm{BAO}$ fits. A06, WV07 and K08 do not explicitly explore the effects of different light-curve/distance fitters on $1+w$, although some of the things they explore are components of an individual fitter's systematic uncertainty. Since probably the largest component of systematic uncertainty is the reddening/color correction, the WV07 estimate implicitly includes much of the systematic uncertainty due to choice of light-curve/distance fitter. Because of this, and as our findings confirm, the WV07 estimate of systematic uncertainty is probably closer to reality than K08 and certainly closer than A06. The majority of the WV07 value comes from three sources: 0.08 from the host galaxy extinction treatment, 0.06 from the uncertainty of the intrinsic color of SN Ia and 0.06 from a potential Hubble bubble. If all three of these are removed then the WV07 uncertainty would be $\Delta_{w}($ syst $)=0.057$, in line with the A06 value, where these effects are not considered.

The choice of which potential sources of systematic error to include and how much of an effect they may have is a somewhat subjective decision but it can be informed by looking at how different samples are affected by different fitters. One measure of the systematic effects is to look at the differences in $1+w$ between the different fitters and samples under the minimal cuts, and again, with best cuts. Our results show the WV07 value seems plausible. The largest difference in $1+w$ for the minimal-cuts OLD+CfA3 sample is 0.12 , between MLCS31 and MLCS17. The host galaxy extinction and the Hubble bubble change significantly by using $R_{V}=1.7$ instead of $R_{V}=3.1$. The uncertainties in these individual effects in WV07 is in rough agreement with our difference in $1+w$ of 0.12 . The largest difference in $1+w$ for the best-cuts OLD+CfA3 sample is 0.09, between MLCS17 and SALT2. The choice of fitter (and the underlying assumptions) is very important. The choice of which SN Ia samples to use is also important. SALT2 and MLCS31 show large differences in $1+w$ between the OLD and the CfA3 samples (-0.15 and -0.13, respectively) while SALT and MLCS17 do not. 
By making cuts on $\Delta$, color and host extinction, a slightly better agreement in $1+w$ is achieved in the OLD+CfA3 samples between MLCS17 and MLCS31 and between SALT and SALT2 but not between either of the SALT/2 cases and either of the MLCS17/31 cases. We note that the best agreement between all four fitters occurs when the color and hostextinction cuts are made without any cuts on $\Delta$. At lower values of host reddening, the influence of the particular MLCS2k2 prior and the value of $R_{V}$ are less important. Similarly, in SALT and SALT2, for the narrower range of color considered, the value of $\beta$ will be more accurate. The difference in $1+w$ between MLCS31 and MLCS17 with $A_{V} \leq 0.5$ is 0.05 , with the SALT and SALT2 values agreeing extremely well with MLCS31, and so we adjust the WV07 uncertainty on $1+w$ due to host-galaxy extinction from 0.08 to 0.05 . The choice of OLD or CfA3 as the nearby sample leads to an average difference in $1+w$ of $0.06-0.07$. By using both, the systematic on $1+w$ should be less and we adopt 0.03 as our value for choice of nearby sample. We also saw in $\S 3.8$ that making cuts on $A_{V}$ made our results even more consistent with no Hubble Bubble and so we reduce our Hubble bubble amplitude uncertainty from 0.02 to 0.01 , reducing the corresponding systematic uncertainty in $1+w$ from 0.06 to 0.03 . Making the cuts on $\Delta$ raises the values of $1+w$ by about 0.04 for both MLCS17 and MLCS31 and so we add this as another component of systematic uncertainty. These three changes result in a systematic uncertainty in $1+w$ of 0.11 for our best-cuts samples. This is roughly 1.4-1.7 times the typical statistical uncertainty of the best-cuts values of $1+w$.

\section{Conclusion}

For people that have used the Union compilation from K08 to perform their own cosmology fits, we recommend that they use our Constitution set (Union+CfA3) in Table 1. The CfA3 sample adds 90 nearby objects to the 57 Union objects. The Constitution set of SN Ia (using the SALT output and a linear luminosity dependence on color and stretch for the cosmology calculation), combined with the BAO prior, give $1+w=0.013_{-0.068}^{+0.066}$, consistent with a cosmological constant. We estimate a systematic uncertainty of 0.11 . Further progress will mostly come by reducing systematic errors and uncertainty. We also invite people to use our SALT2, MLCS31, and MLCS17 light curve fits in Tables 2, 3, and 4, We recommend a cut on $c, \Delta$ and $A_{V}$ as we have employed in $\S 3.11$.

The four fitters are seen to be relatively consistent in the light-curve-shape and color parameters they measure but that there are areas of concern. MLCS2k2 produces negative residuals in the range $0.7<\Delta<1.2$ and when $R_{V}=3.1$ is used the host extinction is overestimated. SALT and SALT2 give rise to different light-curve-shape/luminosity coefficients, 
$\alpha$, at high and low redshift. Both SALT and SALT2 have higher scatter at high redshift. SALT gives rise to a strong trend in residuals versus color at high redshift. SALT2 poorly fits the nearby $U$-band light curves. This shows that light curve and distance fitters still have room for improvement and provide a considerable amount of systematic uncertainty to any analysis. However, by addressing these issues, future iterations of these fitters should be much more robust and agree better with each other.

We have shown by using multiple fitters on the OLD+CfA3 sample, combined with reasonable cuts on color and host reddening, that a consistent value of $1+w$ emerges. However, when we make the additional cut on $\Delta$ in MLCS2k2, which we believe is warranted, the MLCS17 and MLCS31 values of $1+w$ still agree equally well with each other but less well with the SALT and SALT2 values. We consider our best cuts to be $-0.1<c<0.2$ for SALT and SALT2, and $A_{V} \leq 0.5$ and $\Delta<0.7$ for MLCS31 and MLCS17.

Our investigation of the Hubble bubble is consistent with no Hubble bubble to about the 1-2\% level. We also find that SN Ia in E/S0 hosts have negative mean Hubble residuals and low reddening while SN Ia in Scd/Sd/Irr hosts have positive mean Hubble residuals, low scatter and low reddening. After correction for color and light-curve shape, the SN Ia in $\mathrm{Scd} / \mathrm{Sd} / \mathrm{Irr}$ hosts are fainter than those in E/S0 hosts by $2 \sigma$, suggesting that they may come from different populations. It may be worthwhile to form two or more separate samples, based on host-galaxy morphology or color, to train light-curve fitters.

Systematic uncertainties are now the largest obstacle for progress in supernova cosmology, both in better measuring constant $1+w$ and in measuring any time dependence. Three large potential sources of systematic error are SN Ia photometric accuracy, host-galaxy reddening, and SN Ia population differences or evolution. Regarding photometric accuracy, we have broken the nearby sample into subsamples based on the main groups or instruments involved and find that they agree with each other at about the $0.03 \mathrm{mag}$ level. This is an issue which will be improved as the KAIT and Carnegie groups publish their photometry, a good portion of which overlaps with CfA2 and CfA3 photometry. This will allow comparisons and possibly corrections so that the various nearby samples can be combined more consistently. Regarding reddening, the forthcoming sets of near-infrared SN Ia light curves from the CfA and Carnegie groups, combined with optical measurements, should improve our understanding of host-galaxy dust and the intrinsic color variation in SN Ia. As for SN Ia population difference and evolution, continued investigation of the possible two-or-more SN Ia populations should help reduce the systematic uncertainty that may arise. We encourage further efforts in SN Ia data gathering, distance estimation methods and theoretical understanding so that the mystery of the dark energy may be more fully illuminated. 
This work has been supported, in part, by NSF grant AST0606772 to Harvard University. AR thanks the Goldberg Fellowship Program for its support. 


\section{REFERENCES}

Albrect, A., et al. 2006, Report of the Dark Energy Task Force, arXiv:astro-ph/0609591

Albrect, A., et al. 2009, Findings of the Joint Dark Energy Mission Figure of Merit Science Working Group, arXiv:astro-ph/0901.0721

Astier, P., et al. 2006, A\&A, 447, 31

Barris, B., et al. 2004, ApJ, 602, 571

Blondin, S., et al. 2006, AJ, 131, 1648

Blondin, S., et al. 2009, in preparation

Commins, E. D. 2004, NewAR, 48, 567

Conley, A., et al. 2006, ApJ, 644, 1

Conley, A., et al. 2007, ApJ, 664, L13

Conley, A., et al. 2008, ApJ, 681, 482

Davis, T. M., et al. 2007, ApJ, 666, 716

Eisenstein, D. J., et al. 2005, ApJ, 633, 560

Ellis, R. S., et al. 2008, ApJ, 674, 51

Foley, R. J., et al. 2005, ApJ, 626, L11

Foley, R. J., et al. 2008, ApJ, 684, 68

Friedman, A., et al. 2009, in preparation

Gallagher, J. S., et al. 2008, ApJ, 685, 752

Garnavich, P. M., et al. 1998, ApJ, 509, 74

Goldhaber, G., et al. 2001, ApJ, 558, 359

Guy, J., et al. 2005, A\&A 443, 781

Guy, J., et al. 2007, A\&A 466, 11

Hamuy, M., et al. 1991, AJ, 102, 208 
Hamuy, M., et al. 1996, AJ, 112, 2391

Hatano, K., Branch, D., \& Deaton, J. J. 1998, ApJ, 502, 177

Hicken, M., et al. 2009, ApJ, submitted

Hook, I. M., et al. 2005, AJ, 130, 2788

Howell, D. A., et al. 2005, ApJ, 634, 1190

Howell, D. A., et al. 2007, ApJ, 667, L37

Hsiao, E. Y., et al. 2007, ApJ, 663, 1187

Jha, S., et al. 1999, ApJS, 125, 73

Jha, S., et al. 2006, AJ, 131, 527

Jha, S., Riess, A. G., \& Kirshner, R. P. 2007, ApJ, 659, 122

Kowalski, M., et al. 2008, ApJ, 686, 749

Krisciunas, K, et al. 2000, ApJ, 539, 658

Knop, A., et al. 2003, ApJ, 598, 102

Linder, E. V. 2006, Phys. Rev. D, 70, 123516

Mandel, K., et al. 2009, ApJ, submitted

Mannucci, F., Della Valle, M., \& Panagia, N. 2006, MNRAS, 370, 773

Matheson, T., et al. 2005, AJ, 129, 2352

Matheson, T., et al. 2008, AJ, 135, 1598

Miknaitis, G., et al. 2007, ApJ, 666, 674

Monard, L. A. G. 2006, IAUC 8723

Perlmutter, S., et al. 1997, ApJ, 483, 565

Perlmutter, S., et al. 1999, ApJ, 517, 565

Phillips, M. M. 1993, ApJ, 413, L105

Phillips, M. M., et al. 1999, AJ, 118, 1766 
Prieto, J. L., Rest, A., \& Suntzeff, N. B. 2006, ApJ, 647, 501

Riello, M. \& Patat, F. 2005, MNRAS, 362, 671

Riess, A. G., Press, W.H. \& Kirshner, R.P. 1996, AJ, 116, 1009

Riess, A. G., et al. 1998, AJ, 116, 1009

Riess, A. G., et al. 1999, AJ, 11, 707

Riess, A. G., et al. 2000, ApJ, 536, 62

Riess, A. G., et al. 2005, ApJ, 627, 579

Riess, A. G., et al. 2007, ApJ, 659, 98

Scannapieco, E. \& Bildsten, L. 2005, ApJ, 629, L85

Sullivan, M., et al. 2003, MNRAS, 340, 1057

Sullivan, M., et al. 2006, ApJ, 648, 868

Tonry, J. L., et al. 2003, ApJ, 594, 1

Wang, L., et al. 2003, ApJ, 590, 944

Wood-Vasey, M., et al. 2007, ApJ, 666, 694

Wood-Vasey, W. M., et al. 2008, ApJ, 689, 377

Zehavi, I., et al. 1998, ApJ, 503, 483 
Table 1. SALT Light Curve Fit Parameters and Distances. Constitution Set.

\begin{tabular}{|c|c|c|c|c|c|}
\hline SN Ia & $z_{C M B}$ & $m_{B}^{1}$ & $s$ & $c$ & $\mu^{2}$ \\
\hline 1993ag & $0.050(0.001)$ & $17.790(0.108)$ & $0.910(0.020)$ & $0.090(0.020)$ & $36.910(0.135)$ \\
\hline 19930 & $0.053(0.001)$ & $17.610(0.111)$ & $0.900(0.010)$ & $-0.010(0.020)$ & $36.977(0.134)$ \\
\hline $1993 \mathrm{~h}$ & $0.025(0.001)$ & $16.740(0.145)$ & $0.680(0.010)$ & $0.210(0.010)$ & $35.264(0.186)$ \\
\hline 1993b & $0.070(0.001)$ & $18.380(0.040)$ & $0.990(0.030)$ & $0.040(0.040)$ & $37.729(0.124)$ \\
\hline $1992 \mathrm{bs}$ & $0.063(0.001)$ & $18.180(0.108)$ & $1.000(0.020)$ & $-0.030(0.020)$ & $37.722(0.131)$ \\
\hline $1992 \mathrm{br}$ & $0.088(0.001)$ & $19.400(0.001)$ & $0.650(0.040)$ & $0.030(0.050)$ & $38.353(0.143)$ \\
\hline 1992bp & $0.079(0.001)$ & $18.280(0.108)$ & $0.870(0.020)$ & $-0.040(0.020)$ & $37.687(0.128)$ \\
\hline 1992bo & $0.017(0.001)$ & $15.750(0.168)$ & $0.740(0.010)$ & $0.030(0.010)$ & $34.815(0.235)$ \\
\hline 1992bl & $0.042(0.001)$ & $17.290(0.108)$ & $0.790(0.020)$ & $-0.010(0.020)$ & $36.520(0.140)$ \\
\hline 1992bh & $0.045(0.001)$ & $17.590(0.121)$ & $0.980(0.010)$ & $0.100(0.010)$ & $36.771(0.139)$ \\
\hline $1992 \mathrm{bc}$ & $0.020(0.001)$ & $15.070(0.156)$ & $1.010(0.010)$ & $-0.060(0.010)$ & $34.702(0.214)$ \\
\hline $1992 \mathrm{aq}$ & $0.101(0.001)$ & $19.300(0.108)$ & $0.840(0.020)$ & $-0.050(0.020)$ & $38.696(0.126)$ \\
\hline 1992ag & $0.027(0.001)$ & $16.240(0.127)$ & $1.030(0.040)$ & $0.150(0.020)$ & $35.353(0.179)$ \\
\hline 1992ae & $0.075(0.001)$ & $18.390(0.108)$ & $0.940(0.020)$ & $-0.020(0.020)$ & $37.832(0.129)$ \\
\hline $1992 p$ & $0.026(0.001)$ & $16.030(0.107)$ & $1.140(0.080)$ & $-0.010(0.020)$ & $35.694(0.188)$ \\
\hline 1990af & $0.050(0.001)$ & $17.730(0.121)$ & $0.740(0.010)$ & $0.000(0.010)$ & $36.872(0.137)$ \\
\hline 1990 o & $0.031(0.001)$ & $16.200(0.118)$ & $1.040(0.030)$ & $0.000(0.020)$ & $35.714(0.163)$ \\
\hline $2001 \mathrm{cz}$ & $0.016(0.001)$ & $15.030(0.106)$ & $1.010(0.020)$ & $0.110(0.010)$ & $34.222(0.204)$ \\
\hline $2001 \mathrm{cn}$ & $0.015(0.001)$ & $15.220(0.121)$ & $0.920(0.010)$ & $0.170(0.010)$ & $34.145(0.219)$ \\
\hline 2001ba & $0.030(0.001)$ & $16.180(0.013)$ & $1.000(0.010)$ & $-0.040(0.010)$ & $35.748(0.098)$ \\
\hline 2000ca & $0.025(0.001)$ & $15.510(0.053)$ & $1.000(0.030)$ & $-0.060(0.010)$ & $35.130(0.135)$ \\
\hline 2000bh & $0.024(0.001)$ & $15.940(0.040)$ & $0.940(0.040)$ & $0.100(0.020)$ & $35.071(0.143)$ \\
\hline 1999gp & $0.026(0.001)$ & $15.990(0.053)$ & $1.130(0.030)$ & $0.040(0.010)$ & $35.512(0.129)$ \\
\hline $1993 \mathrm{ac}$ & $0.049(0.001)$ & $17.800(0.083)$ & $0.780(0.060)$ & $0.080(0.040)$ & $36.785(0.163)$ \\
\hline $1994 \mathrm{~m}$ & $0.024(0.001)$ & $16.300(0.159)$ & $0.780(0.020)$ & $0.110(0.020)$ & $35.207(0.205)$ \\
\hline $1994 \mathrm{~s}$ & $0.015(0.001)$ & $14.770(0.196)$ & $1.090(0.050)$ & $-0.010(0.020)$ & $34.372(0.280)$ \\
\hline $1994 \mathrm{t}$ & $0.036(0.001)$ & $17.050(0.084)$ & $0.840(0.040)$ & $0.020(0.050)$ & $36.264(0.181)$ \\
\hline $1995 \mathrm{ac}$ & $0.049(0.001)$ & $17.030(0.145)$ & $1.040(0.010)$ & $0.010(0.010)$ & $36.518(0.159)$ \\
\hline 1995ak & $0.022(0.001)$ & $15.870(0.105)$ & $0.890(0.040)$ & $0.070(0.050)$ & $35.017(0.216)$ \\
\hline $1996 \mathrm{c}$ & $0.028(0.001)$ & $16.650(0.147)$ & $1.010(0.020)$ & $0.120(0.020)$ & $35.816(0.188)$ \\
\hline 1996ab & $0.124(0.001)$ & $19.530(0.131)$ & $0.960(0.030)$ & $-0.080(0.020)$ & $39.152(0.148)$ \\
\hline 1996bl & $0.036(0.001)$ & $16.620(0.143)$ & $0.990(0.020)$ & $0.030(0.010)$ & $35.995(0.167)$ \\
\hline $1996 \mathrm{bv}$ & $0.017(0.001)$ & $15.350(0.183)$ & $1.010(0.040)$ & $0.220(0.010)$ & $34.257(0.254)$ \\
\hline 1996bo & $0.016(0.001)$ & $15.820(0.189)$ & $0.870(0.020)$ & $0.370(0.010)$ & $34.165(0.257)$ \\
\hline $2000 \mathrm{fa}$ & $0.022(0.001)$ & $15.840(0.258)$ & $0.960(0.030)$ & $0.080(0.020)$ & $35.048(0.296)$ \\
\hline $2000 \mathrm{dk}$ & $0.016(0.001)$ & $15.330(0.276)$ & $0.730(0.010)$ & $0.050(0.010)$ & $34.330(0.325)$ \\
\hline $2000 \mathrm{cn}$ & $0.023(0.001)$ & $16.530(0.265)$ & $0.730(0.010)$ & $0.170(0.010)$ & $35.219(0.293)$ \\
\hline $2000 \mathrm{cf}$ & $0.036(0.001)$ & $17.090(0.254)$ & $0.880(0.020)$ & $0.050(0.010)$ & $36.276(0.268)$ \\
\hline 1999gd & $0.019(0.001)$ & $16.940(0.274)$ & $0.920(0.030)$ & $0.430(0.010)$ & $35.192(0.313)$ \\
\hline 1999ek & $0.018(0.001)$ & $15.590(0.297)$ & $0.910(0.010)$ & $0.170(0.010)$ & $34.503(0.338)$ \\
\hline $1999 \mathrm{cc}$ & $0.032(0.001)$ & $16.780(0.254)$ & $0.820(0.020)$ & $0.060(0.010)$ & $35.866(0.272)$ \\
\hline 1998 eg & $0.024(0.001)$ & $16.100(0.263)$ & $0.930(0.030)$ & $0.050(0.010)$ & $35.348(0.292)$ \\
\hline $1998 \mathrm{ef}$ & $0.017(0.001)$ & $14.810(0.275)$ & $0.850(0.020)$ & $0.020(0.010)$ & $34.037(0.324)$ \\
\hline $1998 d x$ & $0.054(0.001)$ & $17.640(0.237)$ & $0.750(0.040)$ & $0.080(0.030)$ & $36.587(0.260)$ \\
\hline $1998 \mathrm{co}$ & $0.017(0.001)$ & $15.700(0.274)$ & $0.610(0.030)$ & $0.110(0.010)$ & $34.396(0.322)$ \\
\hline 1998ab & $0.028(0.001)$ & $16.050(0.249)$ & $0.930(0.020)$ & $0.100(0.020)$ & $35.169(0.275)$ \\
\hline
\end{tabular}


Table 1-Continued

\begin{tabular}{|c|c|c|c|c|c|}
\hline SN Ia & $z_{C M B}$ & $m_{B}{ }^{1}$ & $s$ & $c$ & $\mu^{2}$ \\
\hline $1998 v$ & $0.017(0.001)$ & $15.100(0.274)$ & $0.930(0.030)$ & $0.050(0.010)$ & $34.348(0.321)$ \\
\hline $1997 \mathrm{dg}$ & $0.030(0.001)$ & $16.830(0.245)$ & $0.920(0.040)$ & $0.030(0.020)$ & $36.118(0.272)$ \\
\hline $1997 y$ & $0.017(0.001)$ & $15.310(0.275)$ & $0.880(0.020)$ & $0.040(0.010)$ & $34.522(0.324)$ \\
\hline 1999aа & $0.015(0.001)$ & $14.690(0.121)$ & $1.050(0.010)$ & $-0.020(0.010)$ & $34.268(0.223)$ \\
\hline 1999ao & $0.054(0.001)$ & $17.890(0.001)$ & $0.960(0.040)$ & $0.070(0.030)$ & $37.124(0.106)$ \\
\hline 1999ar & $0.156(0.005)$ & $19.970(0.001)$ & $0.990(0.040)$ & $-0.010(0.020)$ & $39.448(0.074)$ \\
\hline 1999aw & $0.039(0.001)$ & $16.780(0.001)$ & $1.200(0.030)$ & $0.010(0.020)$ & $36.467(0.097)$ \\
\hline 1999bi & $0.124(0.001)$ & $19.760(0.001)$ & $1.180(0.050)$ & $0.210(0.020)$ & $38.904(0.084)$ \\
\hline $1999 \mathrm{bm}$ & $0.144(0.001)$ & $20.430(0.001)$ & $0.680(0.030)$ & $0.120(0.020)$ & $39.187(0.067)$ \\
\hline 1999bn & $0.130(0.001)$ & $19.600(0.001)$ & $1.090(0.040)$ & $0.010(0.020)$ & $39.150(0.075)$ \\
\hline 1999bp & $0.078(0.001)$ & $18.390(0.001)$ & $1.090(0.030)$ & $0.000(0.020)$ & $37.966(0.074)$ \\
\hline $1996 \mathrm{~h}$ & $0.620(0.005)$ & $23.500(0.136)$ & $1.100(0.170)$ & $-0.120(0.090)$ & $43.399(0.342)$ \\
\hline $1996 \mathrm{i}$ & $0.570(0.005)$ & $23.400(0.264)$ & $0.820(0.050)$ & $-0.060(0.060)$ & $42.797(0.313)$ \\
\hline $1996 \mathrm{j}$ & $0.300(0.005)$ & $22.030(0.163)$ & $0.790(0.060)$ & $0.070(0.090)$ & $41.053(0.294)$ \\
\hline $1996 \mathrm{k}$ & $0.380(0.005)$ & $22.640(0.240)$ & $0.850(0.010)$ & $-0.100(0.050)$ & $42.178(0.273)$ \\
\hline $1996 \mathrm{u}$ & $0.430(0.005)$ & $22.610(0.211)$ & $0.920(0.070)$ & $-0.210(0.090)$ & $42.519(0.326)$ \\
\hline 1995 ao & $0.240(0.005)$ & $21.600(0.183)$ & $1.350(0.390)$ & $0.180(0.090)$ & $41.032(0.567)$ \\
\hline 1995ap & $0.300(0.005)$ & $21.530(0.001)$ & $0.540(0.200)$ & $0.050(0.320)$ & $40.295(0.865)$ \\
\hline $1996 \mathrm{t}$ & $0.240(0.005)$ & $20.990(0.263)$ & $1.300(0.030)$ & $-0.100(0.010)$ & $41.086(0.267)$ \\
\hline $1997 \mathrm{ce}$ & $0.440(0.005)$ & $22.800(0.251)$ & $0.860(0.030)$ & $-0.040(0.040)$ & $42.195(0.275)$ \\
\hline $1997 \mathrm{cj}$ & $0.500(0.005)$ & $23.140(0.271)$ & $0.910(0.020)$ & $-0.040(0.020)$ & $42.597(0.277)$ \\
\hline $1997 \mathrm{ck}$ & $0.970(0.010)$ & $24.720(0.001)$ & $1.030(0.120)$ & $0.420(0.280)$ & $43.134(0.740)$ \\
\hline $1995 \mathrm{k}$ & $0.479(0.005)$ & $22.720(0.259)$ & $0.980(0.040)$ & $-0.120(0.050)$ & $42.471(0.294)$ \\
\hline 1997ap & $0.830(0.010)$ & $24.340(0.342)$ & $1.000(0.020)$ & $0.060(0.040)$ & $43.649(0.358)$ \\
\hline 1997am & $0.416(0.005)$ & $22.460(0.176)$ & $1.080(0.050)$ & $-0.080(0.140)$ & $42.231(0.408)$ \\
\hline 1997aj & $0.581(0.005)$ & $23.160(0.293)$ & $1.010(0.060)$ & $0.220(0.100)$ & $42.067(0.398)$ \\
\hline 1997ai & $0.450(0.005)$ & $22.920(0.320)$ & $0.740(0.070)$ & $0.050(0.050)$ & $41.933(0.356)$ \\
\hline 1997af & $0.579(0.005)$ & $23.570(0.281)$ & $0.900(0.050)$ & $-0.160(0.170)$ & $43.325(0.526)$ \\
\hline $1997 \mathrm{ac}$ & $0.320(0.005)$ & $21.890(0.318)$ & $1.050(0.020)$ & $0.030(0.030)$ & $41.339(0.328)$ \\
\hline $1997 \mathrm{r}$ & $0.657(0.005)$ & $23.920(0.278)$ & $0.980(0.110)$ & $0.050(0.160)$ & $43.230(0.517)$ \\
\hline $1997 \mathrm{p}$ & $0.472(0.005)$ & $23.130(0.244)$ & $0.940(0.040)$ & $0.110(0.140)$ & $42.235(0.440)$ \\
\hline 1997 o & $0.374(0.005)$ & $23.320(0.001)$ & $1.060(0.060)$ & $-0.180(0.330)$ & $43.325(0.858)$ \\
\hline $1997 \mathrm{~h}$ & $0.526(0.005)$ & $23.180(0.300)$ & $0.930(0.020)$ & $0.170(0.090)$ & $42.117(0.380)$ \\
\hline $1997 \mathrm{~g}$ & $0.763(0.010)$ & $24.370(0.562)$ & $0.900(0.120)$ & $-0.360(0.240)$ & $44.643(0.851)$ \\
\hline $1997 \mathrm{f}$ & $0.580(0.005)$ & $23.410(0.315)$ & $1.110(0.050)$ & $-0.040(0.080)$ & $43.115(0.382)$ \\
\hline $1996 \mathrm{cn}$ & $0.430(0.005)$ & $23.220(0.272)$ & $0.950(0.070)$ & $0.250(0.090)$ & $41.975(0.369)$ \\
\hline $1996 \mathrm{~cm}$ & $0.450(0.005)$ & $23.250(0.271)$ & $0.980(0.040)$ & $0.110(0.110)$ & $42.405(0.396)$ \\
\hline $1996 \mathrm{cl}$ & $0.828(0.010)$ & $24.550(0.001)$ & $1.380(0.560)$ & $0.050(0.170)$ & $44.356(0.822)$ \\
\hline $1996 \mathrm{ck}$ & $0.656(0.005)$ & $23.770(0.330)$ & $0.850(0.090)$ & $-0.130(0.140)$ & $43.385(0.503)$ \\
\hline 1996ci & $0.495(0.005)$ & $22.820(0.300)$ & $0.920(0.090)$ & $-0.040(0.080)$ & $42.289(0.381)$ \\
\hline $1996 \mathrm{cg}$ & $0.490(0.005)$ & $23.070(0.319)$ & $0.980(0.030)$ & $0.230(0.050)$ & $41.914(0.346)$ \\
\hline $1996 \mathrm{cf}$ & $0.570(0.005)$ & $23.310(0.313)$ & $1.080(0.100)$ & $-0.010(0.070)$ & $42.900(0.383)$ \\
\hline $1995 \mathrm{ba}$ & $0.388(0.005)$ & $22.550(0.279)$ & $0.950(0.040)$ & $-0.140(0.080)$ & $42.315(0.351)$ \\
\hline $1995 \mathrm{az}$ & $0.450(0.005)$ & $22.610(0.292)$ & $0.950(0.040)$ & $-0.160(0.080)$ & $42.427(0.361)$ \\
\hline 1995ay & $0.480(0.005)$ & $23.060(0.267)$ & $0.840(0.050)$ & $0.010(0.100)$ & $42.300(0.377)$ \\
\hline 1995ax & $0.615(0.005)$ & $23.220(0.215)$ & $1.120(0.160)$ & $0.020(0.150)$ & $42.782(0.486)$ \\
\hline
\end{tabular}


Table 1-Continued

\begin{tabular}{|c|c|c|c|c|c|}
\hline SN Ia & $z_{C M B}$ & $m_{B}{ }^{1}$ & $s$ & $c$ & $\mu^{2}$ \\
\hline 1995aw & $0.400(0.005)$ & $22.180(0.266)$ & $1.200(0.050)$ & $-0.170(0.090)$ & $42.333(0.359)$ \\
\hline 1995at & $0.655(0.005)$ & $23.220(0.348)$ & $1.150(0.130)$ & $0.160(0.110)$ & $42.456(0.478)$ \\
\hline 1995as & $0.498(0.005)$ & $23.660(0.236)$ & $1.040(0.130)$ & $-0.010(0.120)$ & $43.200(0.422)$ \\
\hline 1995ar & $0.465(0.005)$ & $23.370(0.001)$ & $0.930(0.140)$ & $0.330(0.240)$ & $41.893(0.645)$ \\
\hline $1995 \mathrm{aq}$ & $0.453(0.005)$ & $23.200(0.280)$ & $0.930(0.050)$ & $-0.120(0.090)$ & $42.889(0.369)$ \\
\hline $1994 \mathrm{~g}$ & $0.425(0.005)$ & $22.340(0.140)$ & $0.900(0.100)$ & $0.090(0.110)$ & $41.447(0.341)$ \\
\hline $1999 \mathrm{fw}$ & $0.278(0.005)$ & $21.720(0.001)$ & $0.890(0.100)$ & $0.090(0.060)$ & $40.815(0.199)$ \\
\hline 1999fn & $0.477(0.005)$ & $22.720(0.001)$ & $1.030(0.050)$ & $0.020(0.060)$ & $42.170(0.167)$ \\
\hline $1999 \mathrm{fm}$ & $0.950(0.010)$ & $24.300(0.001)$ & $1.160(0.030)$ & $0.040(0.100)$ & $43.859(0.262)$ \\
\hline 1999fk & $1.057(0.010)$ & $24.770(0.001)$ & $0.950(0.130)$ & $-0.030(0.070)$ & $44.250(0.243)$ \\
\hline 1999fj & $0.816(0.010)$ & $24.220(0.001)$ & $1.020(0.050)$ & $-0.110(0.180)$ & $43.994(0.470)$ \\
\hline 1999ff & $0.455(0.005)$ & $23.210(0.001)$ & $0.860(0.080)$ & $0.000(0.090)$ & $42.501(0.253)$ \\
\hline $2002 \mathrm{ad}$ & $0.514(0.005)$ & $23.060(0.001)$ & $0.890(0.050)$ & $-0.090(0.250)$ & $42.621(0.650)$ \\
\hline $2002 \mathrm{ab}$ & $0.423(0.005)$ & $22.600(0.188)$ & $0.960(0.070)$ & $0.100(0.030)$ & $41.756(0.221)$ \\
\hline 2002aa & $0.946(0.010)$ & $24.600(0.001)$ & $0.960(0.150)$ & $0.300(0.330)$ & $43.238(0.875)$ \\
\hline $2002 x$ & $0.859(0.010)$ & $24.730(0.151)$ & $0.820(0.080)$ & $-0.100(0.080)$ & $44.230(0.275)$ \\
\hline $2002 w$ & $1.031(0.010)$ & $24.470(0.001)$ & $0.950(0.120)$ & $0.670(0.510)$ & $42.137(1.329)$ \\
\hline $2001 \mathrm{kd}$ & $0.936(0.010)$ & $24.960(0.001)$ & $0.640(0.170)$ & $-0.150(0.170)$ & $44.367(0.488)$ \\
\hline 2001jp & $0.528(0.005)$ & $22.890(0.188)$ & $0.940(0.040)$ & $-0.100(0.040)$ & $42.539(0.221)$ \\
\hline $2001 j n$ & $0.645(0.005)$ & $24.550(0.283)$ & $1.330(0.480)$ & $0.100(0.240)$ & $44.165(0.906)$ \\
\hline $2001 \mathrm{jm}$ & $0.978(0.010)$ & $24.500(0.154)$ & $0.850(0.040)$ & $-0.010(0.090)$ & $43.804(0.284)$ \\
\hline 2001jh & $0.885(0.010)$ & $24.310(0.001)$ & $0.940(0.070)$ & $-0.280(0.180)$ & $44.425(0.474)$ \\
\hline $2001 \mathrm{jf}$ & $0.815(0.010)$ & $25.190(0.001)$ & $0.570(0.100)$ & $-0.070(0.420)$ & $44.303(1.095)$ \\
\hline 2001iy & $0.568(0.005)$ & $23.070(0.182)$ & $0.920(0.060)$ & $-0.130(0.070)$ & $42.772(0.268)$ \\
\hline 2001ix & $0.711(0.010)$ & $23.800(0.204)$ & $0.960(0.030)$ & $-0.090(0.040)$ & $43.448(0.232)$ \\
\hline 2001iw & $0.340(0.005)$ & $22.100(0.092)$ & $0.890(0.040)$ & $0.070(0.080)$ & $41.247(0.232)$ \\
\hline $2001 \mathrm{iv}$ & $0.397(0.005)$ & $22.470(0.192)$ & $0.980(0.030)$ & $0.150(0.040)$ & $41.521(0.221)$ \\
\hline 2001hy & $0.812(0.010)$ & $24.950(0.198)$ & $0.710(0.050)$ & $0.030(0.060)$ & $43.977(0.260)$ \\
\hline $2001 \mathrm{hx}$ & $0.799(0.010)$ & $24.780(0.131)$ & $0.960(0.080)$ & $0.380(0.070)$ & $43.211(0.245)$ \\
\hline $2001 \mathrm{hu}$ & $0.882(0.010)$ & $24.910(0.001)$ & $1.130(0.180)$ & $0.630(0.160)$ & $42.904(0.471)$ \\
\hline $2001 \mathrm{hs}$ & $0.833(0.010)$ & $24.260(0.133)$ & $1.090(0.050)$ & $0.120(0.110)$ & $43.525(0.321)$ \\
\hline $2001 \mathrm{fs}$ & $0.874(0.010)$ & $25.120(0.001)$ & $0.840(0.100)$ & $0.370(0.170)$ & $43.428(0.457)$ \\
\hline 2001 fo & $0.772(0.010)$ & $23.750(0.216)$ & $0.960(0.030)$ & $-0.190(0.040)$ & $43.657(0.243)$ \\
\hline $2000 \mathrm{fr}$ & $0.543(0.005)$ & $23.030(0.070)$ & $1.030(0.020)$ & $-0.010(0.030)$ & $42.558(0.108)$ \\
\hline 1998bi & $0.750(0.010)$ & $23.910(0.094)$ & $0.950(0.020)$ & $-0.010(0.020)$ & $43.339(0.110)$ \\
\hline 1998be & $0.640(0.005)$ & $23.800(0.073)$ & $0.730(0.040)$ & $0.020(0.070)$ & $42.878(0.202)$ \\
\hline 1998ba & $0.430(0.005)$ & $22.870(0.001)$ & $0.960(0.040)$ & $-0.030(0.040)$ & $42.363(0.115)$ \\
\hline 1998ay & $0.640(0.005)$ & $23.720(0.075)$ & $1.020(0.050)$ & $-0.060(0.050)$ & $43.365(0.162)$ \\
\hline 1998ax & $0.497(0.005)$ & $23.150(0.069)$ & $1.100(0.030)$ & $0.070(0.040)$ & $42.557(0.130)$ \\
\hline 1998aw & $0.440(0.005)$ & $23.200(0.048)$ & $1.010(0.020)$ & $0.230(0.030)$ & $42.081(0.095)$ \\
\hline 1998as & $0.355(0.005)$ & $22.670(0.001)$ & $0.880(0.020)$ & $0.130(0.040)$ & $41.649(0.107)$ \\
\hline $1997 \mathrm{ez}$ & $0.780(0.010)$ & $24.260(0.076)$ & $1.090(0.040)$ & $0.080(0.030)$ & $43.629(0.120)$ \\
\hline 1997 eq & $0.540(0.005)$ & $23.160(0.060)$ & $0.950(0.020)$ & $-0.020(0.020)$ & $42.614(0.083)$ \\
\hline $1997 \mathrm{ek}$ & $0.860(0.010)$ & $24.480(0.101)$ & $1.000(0.030)$ & $0.020(0.030)$ & $43.893(0.132)$ \\
\hline 04Eag & $1.020(0.010)$ & $24.970(0.097)$ & $0.990(0.050)$ & $0.030(0.060)$ & $44.344(0.193)$ \\
\hline 04Gre & $1.140(0.010)$ & $24.730(0.001)$ & $1.140(0.140)$ & $-0.030(0.080)$ & $44.446(0.270)$ \\
\hline
\end{tabular}


Table 1-Continued

\begin{tabular}{|c|c|c|c|c|c|}
\hline SN Ia & $z_{C M B}$ & $m_{B}{ }^{1}$ & $s$ & $c$ & $\mu^{2}$ \\
\hline 04Man & $0.854(0.010)$ & $24.530(0.087)$ & $0.940(0.060)$ & $-0.070(0.060)$ & $44.102(0.193)$ \\
\hline $04 \mathrm{Mcg}$ & $1.370(0.010)$ & $25.730(0.001)$ & $0.910(0.190)$ & $0.060(0.100)$ & $44.928(0.350)$ \\
\hline $04 \mathrm{Omb}$ & $0.975(0.010)$ & $24.880(0.141)$ & $1.220(0.060)$ & $0.100(0.030)$ & $44.358(0.178)$ \\
\hline 04Pat & $0.970(0.010)$ & $25.020(0.095)$ & $0.990(0.090)$ & $-0.120(0.060)$ & $44.783(0.214)$ \\
\hline 04Rak & $0.740(0.010)$ & $23.840(0.105)$ & $1.020(0.040)$ & $-0.110(0.050)$ & $43.614(0.174)$ \\
\hline 04Sas & $1.390(0.010)$ & $25.820(0.001)$ & $1.390(0.640)$ & $0.340(0.170)$ & $44.888(0.908)$ \\
\hline 04Yow & $0.460(0.005)$ & $23.590(0.001)$ & $0.860(0.070)$ & $0.240(0.060)$ & $42.259(0.178)$ \\
\hline 05Fer & $1.020(0.010)$ & $24.830(0.001)$ & $1.080(0.100)$ & $0.110(0.070)$ & $44.109(0.220)$ \\
\hline $05 \mathrm{Gab}$ & $1.120(0.010)$ & $25.070(0.092)$ & $0.960(0.050)$ & $0.030(0.070)$ & $44.407(0.213)$ \\
\hline 05Lan & $1.230(0.010)$ & $26.020(0.077)$ & $0.880(0.100)$ & $0.140(0.060)$ & $44.973(0.213)$ \\
\hline 05Red & $1.190(0.010)$ & $25.760(0.139)$ & $0.700(0.100)$ & $0.250(0.050)$ & $44.205(0.227)$ \\
\hline 05Spo & $0.839(0.010)$ & $24.200(0.001)$ & $1.000(0.050)$ & $-0.040(0.070)$ & $43.768(0.192)$ \\
\hline 05Str & $1.010(0.010)$ & $25.030(0.165)$ & $1.080(0.040)$ & $-0.010(0.020)$ & $44.620(0.180)$ \\
\hline 05Zwi & $0.521(0.005)$ & $23.070(0.001)$ & $1.210(0.060)$ & $0.090(0.060)$ & $42.562(0.172)$ \\
\hline $2002 \mathrm{dc}$ & $0.475(0.005)$ & $23.090(0.001)$ & $0.830(0.050)$ & $0.000(0.130)$ & $42.344(0.342)$ \\
\hline 2002dd & $0.950(0.010)$ & $24.660(0.083)$ & $1.090(0.080)$ & $0.130(0.050)$ & $43.900(0.183)$ \\
\hline $2002 \mathrm{fw}$ & $1.300(0.010)$ & $25.650(0.091)$ & $1.020(0.090)$ & $0.010(0.110)$ & $45.114(0.319)$ \\
\hline $2002 \mathrm{hp}$ & $1.305(0.010)$ & $25.410(0.158)$ & $0.860(0.040)$ & $0.050(0.080)$ & $44.572(0.265)$ \\
\hline $2002 \mathrm{hr}$ & $0.526(0.005)$ & $24.040(0.114)$ & $1.030(0.090)$ & $0.160(0.050)$ & $43.127(0.205)$ \\
\hline $2002 \mathrm{kd}$ & $0.735(0.010)$ & $24.020(0.113)$ & $0.920(0.010)$ & $0.040(0.040)$ & $43.282(0.154)$ \\
\hline $2002 \mathrm{ki}$ & $1.140(0.010)$ & $25.350(0.240)$ & $1.000(0.360)$ & $0.270(0.200)$ & $44.115(0.725)$ \\
\hline $2003 \mathrm{ak}$ & $1.551(0.010)$ & $26.640(0.001)$ & $1.040(0.460)$ & $0.290(0.080)$ & $45.403(0.607)$ \\
\hline $2003 \mathrm{az}$ & $1.265(0.010)$ & $25.680(0.135)$ & $0.990(0.070)$ & $0.150(0.050)$ & $44.744(0.206)$ \\
\hline 2003dy & $1.340(0.010)$ & $25.770(0.001)$ & $1.230(0.150)$ & $0.180(0.070)$ & $45.054(0.260)$ \\
\hline 2003eq & $0.840(0.010)$ & $24.350(0.148)$ & $0.970(0.050)$ & $0.020(0.030)$ & $43.726(0.178)$ \\
\hline 03D4au & $0.468(0.005)$ & $23.860(0.069)$ & $1.000(0.030)$ & $0.290(0.040)$ & $42.574(0.130)$ \\
\hline 04D4bk & $0.840(0.010)$ & $24.310(0.001)$ & $1.050(0.060)$ & $0.140(0.120)$ & $43.474(0.320)$ \\
\hline 04D3nr & $0.960(0.010)$ & $24.540(0.143)$ & $0.920(0.060)$ & $0.070(0.140)$ & 43.724(0.397) \\
\hline 04D3lu & $0.822(0.010)$ & $24.340(0.052)$ & $0.950(0.030)$ & $0.020(0.120)$ & $43.691(0.317)$ \\
\hline 04D3ki & $0.930(0.010)$ & $24.870(0.237)$ & $0.900(0.050)$ & $-0.260(0.230)$ & $44.884(0.644)$ \\
\hline 04D3gt & $0.451(0.005)$ & $23.230(0.097)$ & $0.950(0.010)$ & $0.280(0.020)$ & $41.907(0.111)$ \\
\hline 04D3do & $0.610(0.005)$ & $23.570(0.089)$ & $0.860(0.020)$ & $-0.080(0.030)$ & $43.068(0.121)$ \\
\hline 04D3cp & $0.830(0.010)$ & $24.240(0.001)$ & $1.110(0.030)$ & $-0.450(0.170)$ & $45.007(0.442)$ \\
\hline 04D2gp & $0.707(0.010)$ & $24.150(0.043)$ & $0.800(0.050)$ & $-0.050(0.080)$ & $43.496(0.220)$ \\
\hline 04D2fp & $0.415(0.005)$ & $22.530(0.081)$ & $0.960(0.010)$ & $0.010(0.020)$ & $41.919(0.098)$ \\
\hline 04D1ag & $0.557(0.005)$ & $23.000(0.084)$ & $0.940(0.030)$ & $-0.180(0.030)$ & $42.856(0.120)$ \\
\hline 03D4fd & $0.791(0.010)$ & $24.210(0.001)$ & $0.940(0.050)$ & $0.040(0.060)$ & $43.497(0.167)$ \\
\hline $03 \mathrm{D} 4 \mathrm{cz}$ & $0.695(0.005)$ & $24.030(0.068)$ & $0.750(0.030)$ & $-0.060(0.050)$ & $43.340(0.151)$ \\
\hline 03D4at & $0.633(0.005)$ & $23.740(0.001)$ & $0.980(0.060)$ & $-0.080(0.060)$ & $43.387(0.172)$ \\
\hline 03D3bh & $0.249(0.005)$ & $21.130(0.001)$ & $0.990(0.030)$ & $-0.090(0.060)$ & $40.815(0.160)$ \\
\hline 03D3af & $0.532(0.005)$ & $23.490(0.105)$ & $0.940(0.040)$ & $0.030(0.050)$ & $42.803(0.174)$ \\
\hline 03D1fc & $0.331(0.005)$ & $21.800(0.080)$ & $0.940(0.010)$ & $0.040(0.010)$ & $41.087(0.086)$ \\
\hline 03D1bp & $0.346(0.005)$ & $22.450(0.053)$ & $0.840(0.010)$ & $0.120(0.030)$ & $41.405(0.095)$ \\
\hline 04D4dw & $0.961(0.010)$ & $24.570(0.001)$ & $0.960(0.080)$ & $-0.120(0.190)$ & $44.296(0.502)$ \\
\hline 04D4an & $0.613(0.005)$ & $24.020(0.108)$ & $0.820(0.020)$ & $0.060(0.020)$ & $43.106(0.123)$ \\
\hline 04D3nh & $0.340(0.005)$ & $22.140(0.080)$ & $1.010(0.010)$ & $0.080(0.010)$ & $41.410(0.086)$ \\
\hline
\end{tabular}


Table 1-Continued

\begin{tabular}{|c|c|c|c|c|c|}
\hline SN Ia & $z_{C M B}$ & $m_{B}^{1}$ & $s$ & $c$ & $\mu^{2}$ \\
\hline 04D3lp & $0.983(0.010)$ & $24.930(0.371)$ & $0.830(0.060)$ & $0.020(0.280)$ & $44.132(0.818)$ \\
\hline 04D3is & $0.710(0.010)$ & $24.260(0.084)$ & $0.980(0.040)$ & $0.220(0.050)$ & $43.130(0.162)$ \\
\hline 04D3fq & $0.730(0.010)$ & $24.130(0.090)$ & $0.920(0.030)$ & $0.010(0.040)$ & $43.469(0.142)$ \\
\hline 04D3df & $0.470(0.005)$ & $23.470(0.089)$ & $0.730(0.020)$ & $0.060(0.030)$ & $42.444(0.121)$ \\
\hline 04D3co & $0.620(0.005)$ & $23.780(0.089)$ & $0.890(0.020)$ & $-0.060(0.030)$ & $43.264(0.121)$ \\
\hline 04D2gc & $0.521(0.005)$ & $23.320(0.073)$ & $1.070(0.050)$ & $0.180(0.040)$ & $42.405(0.141)$ \\
\hline 04D2cf & $0.369(0.005)$ & $22.340(0.092)$ & $0.890(0.020)$ & $0.000(0.010)$ & $41.668(0.099)$ \\
\hline 03D4gl & $0.571(0.005)$ & $23.260(0.001)$ & $0.980(0.060)$ & $0.030(0.050)$ & $42.622(0.149)$ \\
\hline 03D4dy & $0.604(0.005)$ & $23.320(0.094)$ & $1.060(0.020)$ & $0.110(0.020)$ & $42.574(0.110)$ \\
\hline 03D4cy & $0.927(0.010)$ & $24.720(0.175)$ & $1.050(0.060)$ & $-0.290(0.210)$ & $44.998(0.576)$ \\
\hline 03D4ag & $0.285(0.005)$ & $21.210(0.092)$ & $1.020(0.020)$ & $-0.080(0.010)$ & $40.907(0.099)$ \\
\hline 03D3ba & $0.291(0.005)$ & $22.050(0.073)$ & $1.040(0.030)$ & $0.260(0.020)$ & $40.891(0.097)$ \\
\hline 03D1gt & $0.548(0.005)$ & $24.120(0.001)$ & $0.860(0.050)$ & $0.240(0.060)$ & $42.789(0.167)$ \\
\hline 03D1ew & $0.868(0.010)$ & $24.370(0.001)$ & $1.020(0.060)$ & $-0.110(0.250)$ & $44.144(0.652)$ \\
\hline 03D1ax & $0.496(0.005)$ & $22.960(0.075)$ & $0.880(0.020)$ & $-0.050(0.040)$ & $42.405(0.130)$ \\
\hline 04D4dm & $0.811(0.010)$ & $24.390(0.001)$ & $1.000(0.050)$ & $-0.160(0.140)$ & $44.269(0.368)$ \\
\hline 04D3oe & $0.756(0.010)$ & $24.080(0.060)$ & $0.810(0.030)$ & $-0.230(0.060)$ & $43.905(0.171)$ \\
\hline 04D3nc & $0.817(0.010)$ & $24.270(0.001)$ & $1.110(0.050)$ & $0.060(0.110)$ & $43.716(0.292)$ \\
\hline 04D3ks & $0.752(0.010)$ & $23.880(0.058)$ & $1.010(0.040)$ & $0.020(0.050)$ & $43.305(0.150)$ \\
\hline 04D3hn & $0.552(0.005)$ & $23.470(0.089)$ & $0.900(0.020)$ & $0.110(0.030)$ & $42.526(0.121)$ \\
\hline 04D3fk & $0.358(0.005)$ & $22.530(0.096)$ & $0.920(0.000)$ & $0.150(0.010)$ & $41.507(0.099)$ \\
\hline 04D3dd & $1.010(0.010)$ & $25.120(0.445)$ & $1.090(0.100)$ & $-0.070(0.280)$ & $44.877(0.860)$ \\
\hline 04D2ja & $0.741(0.010)$ & $24.100(0.105)$ & $0.950(0.020)$ & $-0.070(0.030)$ & $43.684(0.133)$ \\
\hline 04D2gb & $0.430(0.005)$ & $22.800(0.070)$ & $0.780(0.020)$ & $-0.010(0.030)$ & $42.018(0.108)$ \\
\hline 04D1ak & $0.526(0.005)$ & $23.630(0.040)$ & $0.820(0.030)$ & $0.020(0.040)$ & $42.820(0.117)$ \\
\hline 03D4gg & $0.592(0.005)$ & $23.400(0.054)$ & $1.000(0.080)$ & $0.080(0.050)$ & $42.657(0.172)$ \\
\hline 03D4di & $0.905(0.010)$ & $24.290(0.117)$ & $1.100(0.050)$ & $0.020(0.140)$ & $43.827(0.386)$ \\
\hline $03 \mathrm{D} 4 \mathrm{cx}$ & $0.949(0.010)$ & $24.500(0.184)$ & $0.900(0.060)$ & $0.090(0.150)$ & $43.608(0.436)$ \\
\hline 03D3cd & $0.461(0.005)$ & $22.560(0.001)$ & $1.130(0.180)$ & $0.020(0.060)$ & $42.134(0.272)$ \\
\hline 03D3ay & $0.371(0.005)$ & $22.200(0.078)$ & $0.970(0.020)$ & $-0.020(0.020)$ & $41.679(0.097)$ \\
\hline 03D1fq & $0.800(0.010)$ & $24.520(0.001)$ & $0.770(0.060)$ & $-0.390(0.210)$ & $44.709(0.549)$ \\
\hline 03D1co & $0.679(0.005)$ & $24.100(0.068)$ & $1.010(0.030)$ & $0.000(0.050)$ & $43.577(0.151)$ \\
\hline 03D1aw & $0.582(0.005)$ & $23.590(0.001)$ & $0.980(0.040)$ & $0.010(0.060)$ & $43.004(0.163)$ \\
\hline 04D4bq & $0.550(0.005)$ & $23.360(0.059)$ & $1.000(0.040)$ & $0.110(0.040)$ & $42.540(0.129)$ \\
\hline 04D3ny & $0.810(0.010)$ & $24.270(0.001)$ & $1.010(0.100)$ & $-0.070(0.180)$ & $43.928(0.482)$ \\
\hline 04D3ml & $0.950(0.010)$ & $24.550(0.001)$ & $1.190(0.150)$ & $0.130(0.160)$ & $43.913(0.454)$ \\
\hline 04D3kr & $0.337(0.005)$ & $21.970(0.080)$ & $1.060(0.010)$ & $0.070(0.010)$ & $41.328(0.086)$ \\
\hline 04D3gx & $0.910(0.010)$ & $24.710(0.109)$ & $0.950(0.040)$ & $-0.200(0.130)$ & $44.631(0.357)$ \\
\hline 04D3ez & $0.263(0.005)$ & $21.680(0.077)$ & $0.890(0.020)$ & $0.090(0.010)$ & $40.775(0.086)$ \\
\hline 04D3cy & $0.643(0.005)$ & $23.800(0.075)$ & $0.960(0.020)$ & $0.020(0.040)$ & $43.163(0.130)$ \\
\hline 04D2iu & $0.691(0.005)$ & $24.260(0.094)$ & $0.800(0.020)$ & $0.070(0.040)$ & $43.295(0.142)$ \\
\hline 04D2fs & $0.357(0.005)$ & $22.420(0.078)$ & $0.940(0.020)$ & $0.130(0.020)$ & $41.474(0.097)$ \\
\hline 04D1aj & $0.721(0.010)$ & $23.900(0.001)$ & $1.070(0.090)$ & $0.070(0.050)$ & $43.270(0.171)$ \\
\hline 03D4gf & $0.581(0.005)$ & $23.350(0.084)$ & $1.020(0.030)$ & $-0.050(0.030)$ & $42.969(0.120)$ \\
\hline 03D4dh & $0.627(0.005)$ & $23.390(0.108)$ & $1.040(0.020)$ & $0.010(0.020)$ & $42.878(0.123)$ \\
\hline 03D4cn & $0.818(0.010)$ & $24.650(0.001)$ & $0.750(0.080)$ & $0.030(0.210)$ & $43.727(0.553)$ \\
\hline
\end{tabular}


Table 1-Continued

\begin{tabular}{|c|c|c|c|c|c|}
\hline SN Ia & $z_{C M B}$ & $m_{B}^{1}$ & $s$ & $c$ & $\mu^{2}$ \\
\hline 03D3aw & $0.449(0.005)$ & $22.550(0.089)$ & $0.950(0.020)$ & $-0.050(0.030)$ & $42.082(0.121)$ \\
\hline 03D1fl & $0.688(0.005)$ & $23.630(0.094)$ & $0.960(0.050)$ & $-0.070(0.040)$ & $43.226(0.153)$ \\
\hline $03 \mathrm{D} 1 \mathrm{~cm}$ & $0.870(0.010)$ & $24.460(0.116)$ & $1.190(0.060)$ & $-0.040(0.170)$ & $44.264(0.461)$ \\
\hline 03D1au & $0.504(0.005)$ & $22.980(0.070)$ & $1.080(0.020)$ & $0.030(0.030)$ & $42.466(0.108)$ \\
\hline b010 & $0.591(0.005)$ & $23.400(0.052)$ & $1.240(0.090)$ & $-0.140(0.080)$ & $43.525(0.241)$ \\
\hline b013 & $0.426(0.005)$ & $22.680(0.153)$ & $1.010(0.040)$ & $0.080(0.030)$ & $41.950(0.179)$ \\
\hline b016 & $0.329(0.005)$ & $22.500(0.001)$ & $1.140(0.110)$ & $0.260(0.090)$ & $41.465(0.270)$ \\
\hline $\mathrm{d} 033$ & $0.531(0.005)$ & $23.230(0.096)$ & $1.150(0.120)$ & $-0.150(0.080)$ & $43.269(0.273)$ \\
\hline d058 & $0.583(0.005)$ & $23.590(0.001)$ & $1.090(0.210)$ & $0.180(0.150)$ & $42.700(0.468)$ \\
\hline d084 & $0.519(0.005)$ & $23.640(0.001)$ & $1.090(0.170)$ & $-0.010(0.140)$ & $43.242(0.419)$ \\
\hline d085 & $0.401(0.005)$ & $22.480(0.071)$ & $1.020(0.060)$ & $0.080(0.080)$ & $41.762(0.231)$ \\
\hline d087 & $0.340(0.005)$ & $21.910(0.001)$ & $0.990(0.070)$ & $0.040(0.080)$ & $41.259(0.225)$ \\
\hline d089 & $0.436(0.005)$ & $22.500(0.139)$ & $1.040(0.040)$ & $0.000(0.050)$ & $42.014(0.197)$ \\
\hline d093 & $0.363(0.005)$ & $21.890(0.088)$ & $1.040(0.030)$ & $-0.070(0.060)$ & $41.586(0.183)$ \\
\hline d097 & $0.436(0.005)$ & $22.500(0.108)$ & $1.260(0.060)$ & $0.090(0.050)$ & $42.054(0.185)$ \\
\hline $\mathrm{d} 117$ & $0.309(0.005)$ & $22.360(0.093)$ & $0.830(0.020)$ & $0.070(0.060)$ & $41.432(0.183)$ \\
\hline d149 & $0.342(0.005)$ & $22.190(0.138)$ & $1.010(0.030)$ & $0.070(0.040)$ & $41.486(0.177)$ \\
\hline e029 & $0.332(0.005)$ & $22.520(0.087)$ & $0.840(0.060)$ & $0.170(0.060)$ & $41.346(0.193)$ \\
\hline e108 & $0.469(0.005)$ & $22.550(0.134)$ & $1.100(0.050)$ & $-0.050(0.050)$ & $42.268(0.196)$ \\
\hline e132 & $0.239(0.005)$ & $21.700(0.127)$ & $0.920(0.020)$ & $0.190(0.040)$ & $40.573(0.166)$ \\
\hline e136 & $0.352(0.005)$ & $22.800(0.097)$ & $0.820(0.050)$ & $0.190(0.060)$ & $41.549(0.193)$ \\
\hline e138 & $0.612(0.005)$ & $24.050(0.001)$ & $1.370(0.190)$ & $0.680(0.310)$ & $42.212(0.837)$ \\
\hline e140 & $0.631(0.005)$ & $23.390(0.106)$ & $0.990(0.100)$ & $0.050(0.070)$ & $42.713(0.244)$ \\
\hline e147 & $0.645(0.005)$ & $23.380(0.124)$ & $0.960(0.040)$ & $-0.090(0.060)$ & $43.028(0.205)$ \\
\hline e148 & $0.429(0.005)$ & $22.650(0.143)$ & $0.870(0.030)$ & $-0.020(0.050)$ & $42.005(0.197)$ \\
\hline e149 & $0.497(0.005)$ & $22.900(0.081)$ & $0.920(0.030)$ & $0.010(0.070)$ & $42.239(0.202)$ \\
\hline f011 & $0.539(0.005)$ & $23.290(0.092)$ & $0.880(0.050)$ & $0.030(0.070)$ & $42.528(0.213)$ \\
\hline f041 & $0.561(0.005)$ & $23.090(0.097)$ & $1.070(0.070)$ & $-0.050(0.070)$ & $42.771(0.223)$ \\
\hline f076 & $0.410(0.005)$ & $22.370(0.110)$ & $0.900(0.030)$ & $0.090(0.060)$ & $41.477(0.194)$ \\
\hline f096 & $0.412(0.005)$ & $23.060(0.001)$ & $1.210(0.100)$ & $0.370(0.120)$ & $41.827(0.335)$ \\
\hline $\mathrm{f} 216$ & $0.599(0.005)$ & $23.750(0.092)$ & $0.710(0.060)$ & $-0.070(0.090)$ & $43.036(0.261)$ \\
\hline $\mathrm{f} 231$ & $0.619(0.005)$ & $23.450(0.097)$ & $1.010(0.070)$ & $-0.070(0.070)$ & $43.108(0.223)$ \\
\hline $\mathrm{f} 235$ & $0.422(0.005)$ & $22.450(0.001)$ & $0.880(0.050)$ & $-0.030(0.080)$ & $41.843(0.216)$ \\
\hline $\mathrm{f} 244$ & $0.540(0.005)$ & $23.300(0.085)$ & $0.940(0.110)$ & $-0.020(0.060)$ & $42.742(0.224)$ \\
\hline f308 & $0.401(0.005)$ & $23.070(0.001)$ & $0.880(0.140)$ & $0.070(0.100)$ & $42.205(0.312)$ \\
\hline g005 & $0.218(0.005)$ & $21.320(0.087)$ & $1.170(0.060)$ & $0.230(0.050)$ & $40.400(0.173)$ \\
\hline g050 & $0.633(0.005)$ & $23.180(0.110)$ & $0.960(0.070)$ & $0.090(0.090)$ & $42.362(0.272)$ \\
\hline g052 & $0.383(0.005)$ & $22.330(0.001)$ & $0.780(0.030)$ & $-0.100(0.080)$ & $41.781(0.211)$ \\
\hline g055 & $0.302(0.005)$ & $23.280(0.001)$ & $1.230(0.130)$ & $0.570(0.110)$ & $41.553(0.327)$ \\
\hline g097 & $0.340(0.005)$ & $22.270(0.138)$ & $1.000(0.030)$ & $0.190(0.040)$ & $41.242(0.177)$ \\
\hline g120 & $0.510(0.005)$ & $22.790(0.071)$ & $0.960(0.060)$ & $0.060(0.080)$ & $42.050(0.231)$ \\
\hline g133 & $0.421(0.005)$ & $23.170(0.001)$ & $1.150(0.110)$ & $0.300(0.060)$ & $42.044(0.207)$ \\
\hline g142 & $0.399(0.005)$ & $23.460(0.001)$ & $0.750(0.090)$ & $0.320(0.110)$ & $41.786(0.306)$ \\
\hline g160 & $0.493(0.005)$ & $22.920(0.073)$ & $1.060(0.040)$ & $0.080(0.070)$ & $42.252(0.202)$ \\
\hline g240 & $0.687(0.005)$ & $23.400(0.117)$ & $0.920(0.070)$ & $-0.100(0.080)$ & $43.024(0.253)$ \\
\hline h283 & $0.502(0.005)$ & $23.450(0.001)$ & $0.650(0.100)$ & $0.180(0.160)$ & $42.014(0.433)$ \\
\hline
\end{tabular}


Table 1-Continued

\begin{tabular}{|c|c|c|c|c|c|}
\hline SN Ia & $z_{C M B}$ & $m_{B}{ }^{1}$ & $s$ & $c$ & $\mu^{2}$ \\
\hline h300 & $0.687(0.005)$ & $23.520(0.153)$ & $1.040(0.050)$ & $0.020(0.060)$ & $42.982(0.227)$ \\
\hline h319 & $0.495(0.005)$ & $22.900(0.130)$ & $1.090(0.080)$ & $0.050(0.060)$ & $42.347(0.226)$ \\
\hline h323 & $0.603(0.005)$ & $23.480(0.111)$ & $0.920(0.050)$ & $0.020(0.080)$ & $42.794(0.243)$ \\
\hline h342 & $0.421(0.005)$ & $22.440(0.001)$ & $1.150(0.110)$ & $-0.050(0.090)$ & $42.220(0.270)$ \\
\hline h359 & $0.348(0.005)$ & $22.650(0.138)$ & $0.950(0.030)$ & $0.150(0.040)$ & $41.664(0.177)$ \\
\hline h363 & $0.213(0.005)$ & $22.010(0.001)$ & $0.880(0.030)$ & $0.360(0.070)$ & $40.393(0.186)$ \\
\hline h364 & $0.344(0.005)$ & $21.710(0.141)$ & $0.900(0.020)$ & $-0.080(0.040)$ & $41.258(0.177)$ \\
\hline k396 & $0.271(0.005)$ & $21.840(0.057)$ & $0.810(0.060)$ & $0.150(0.060)$ & $40.681(0.182)$ \\
\hline $\mathrm{k} 411$ & $0.564(0.005)$ & $22.890(0.180)$ & $0.930(0.160)$ & $-0.120(0.100)$ & $42.579(0.373)$ \\
\hline $\mathrm{k} 425$ & $0.274(0.005)$ & $21.940(0.029)$ & $0.910(0.040)$ & $0.150(0.070)$ & $40.904(0.190)$ \\
\hline $\mathrm{k} 430$ & $0.582(0.005)$ & $23.810(0.126)$ & $0.940(0.060)$ & $-0.080(0.080)$ & $43.407(0.254)$ \\
\hline $\mathrm{k} 441$ & $0.680(0.005)$ & $23.730(0.104)$ & $1.130(0.100)$ & $0.160(0.060)$ & $42.941(0.224)$ \\
\hline $\mathrm{k} 448$ & $0.401(0.005)$ & $23.340(0.082)$ & $0.970(0.060)$ & $0.290(0.070)$ & $42.016(0.212)$ \\
\hline $\mathrm{k} 485$ & $0.416(0.005)$ & $23.930(0.001)$ & $0.920(0.120)$ & $0.610(0.110)$ & $41.715(0.321)$ \\
\hline $\mathrm{m} 027$ & $0.286(0.005)$ & $22.520(0.001)$ & $1.100(0.190)$ & $0.240(0.100)$ & $41.487(0.350)$ \\
\hline m062 & $0.314(0.005)$ & $21.990(0.001)$ & $0.830(0.130)$ & $0.030(0.080)$ & $41.166(0.263)$ \\
\hline m138 & $0.581(0.005)$ & $23.280(0.233)$ & $1.200(0.110)$ & $-0.340(0.090)$ & $43.873(0.357)$ \\
\hline $\mathrm{m} 158$ & $0.463(0.005)$ & $23.090(0.111)$ & $1.000(0.050)$ & $0.190(0.080)$ & $42.062(0.243)$ \\
\hline m193 & $0.341(0.005)$ & $21.660(0.128)$ & $0.980(0.030)$ & $-0.070(0.050)$ & $41.281(0.186)$ \\
\hline $\mathrm{m} 226$ & $0.671(0.005)$ & $23.640(0.001)$ & $1.070(0.150)$ & $0.370(0.140)$ & $42.233(0.408)$ \\
\hline $\mathrm{n} 256$ & $0.631(0.005)$ & $23.410(0.126)$ & $1.070(0.080)$ & $-0.060(0.050)$ & $43.117(0.206)$ \\
\hline n258 & $0.522(0.005)$ & $23.290(0.001)$ & $0.910(0.050)$ & $-0.080(0.110)$ & $42.850(0.292)$ \\
\hline n263 & $0.368(0.005)$ & $22.040(0.138)$ & $0.970(0.030)$ & $-0.050(0.040)$ & $41.597(0.177)$ \\
\hline n278 & $0.309(0.005)$ & $21.870(0.123)$ & $0.890(0.040)$ & $0.080(0.050)$ & $40.991(0.185)$ \\
\hline n285 & $0.528(0.005)$ & $23.270(0.001)$ & $1.140(0.220)$ & $0.110(0.100)$ & $42.623(0.376)$ \\
\hline n326 & $0.268(0.005)$ & $22.110(0.114)$ & $0.660(0.020)$ & $0.090(0.050)$ & $40.920(0.175)$ \\
\hline $\mathrm{p} 454$ & $0.695(0.005)$ & $23.930(0.001)$ & $0.710(0.160)$ & $-0.020(0.150)$ & $43.087(0.436)$ \\
\hline $\mathrm{p} 455$ & $0.284(0.005)$ & $21.660(0.114)$ & $0.850(0.020)$ & $-0.010(0.050)$ & $40.965(0.175)$ \\
\hline p524 & $0.508(0.005)$ & $22.910(0.152)$ & $1.020(0.030)$ & $0.040(0.040)$ & $42.296(0.188)$ \\
\hline p528 & $0.781(0.010)$ & $24.120(0.001)$ & $0.960(0.160)$ & $0.000(0.160)$ & $43.535(0.459)$ \\
\hline p534 & $0.613(0.005)$ & $23.400(0.001)$ & $0.630(0.320)$ & $-0.080(0.180)$ & $42.613(0.612)$ \\
\hline $\mathrm{sn} 01 \mathrm{G}$ & $0.017(0.001)$ & $14.857(0.140)$ & $0.892(0.009)$ & $-0.037(0.017)$ & $34.285(0.218)$ \\
\hline sn01ah & $0.058(0.001)$ & $17.582(0.139)$ & $1.098(0.023)$ & $0.029(0.011)$ & $37.092(0.153)$ \\
\hline sn01az & $0.041(0.001)$ & $16.983(0.140)$ & $0.934(0.055)$ & $0.004(0.015)$ & $36.355(0.175)$ \\
\hline sn01bf & $0.015(0.001)$ & $14.730(0.138)$ & $1.031(0.006)$ & $0.067(0.004)$ & $34.059(0.229)$ \\
\hline sn01cp & $0.022(0.001)$ & $15.425(0.139)$ & $1.141(0.000)$ & $-0.148(0.012)$ & $35.447(0.190)$ \\
\hline sn01da & $0.016(0.001)$ & $15.346(0.138)$ & $0.908(0.001)$ & $0.098(0.011)$ & $34.443(0.224)$ \\
\hline sn01eh & $0.036(0.001)$ & $16.568(0.138)$ & $1.188(0.010)$ & $0.033(0.005)$ & $36.180(0.160)$ \\
\hline sn02bf & $0.025(0.001)$ & $16.584(0.138)$ & $0.731(0.005)$ & $0.272(0.008)$ & $35.010(0.180)$ \\
\hline sn02ck & $0.030(0.001)$ & $16.305(0.140)$ & $0.974(0.006)$ & $0.029(0.007)$ & $35.661(0.170)$ \\
\hline sn02de & $0.028(0.001)$ & $16.651(0.138)$ & $1.015(0.023)$ & $0.177(0.007)$ & $35.675(0.174)$ \\
\hline sn02do & $0.015(0.001)$ & $15.576(0.139)$ & $0.615(0.006)$ & $0.096(0.007)$ & $34.315(0.230)$ \\
\hline $\mathrm{sn} 02 \mathrm{G}$ & $0.035(0.001)$ & $17.520(0.144)$ & $0.801(0.032)$ & $0.255(0.022)$ & $36.077(0.180)$ \\
\hline sn02hd & $0.036(0.001)$ & $16.586(0.142)$ & $0.860(0.019)$ & $0.047(0.011)$ & $35.757(0.167)$ \\
\hline sn02he & $0.025(0.001)$ & $16.230(0.139)$ & $0.778(0.008)$ & $0.042(0.007)$ & $35.311(0.181)$ \\
\hline sn02hu & $0.038(0.001)$ & $16.607(0.138)$ & $1.009(0.007)$ & $-0.026(0.004)$ & $36.150(0.158)$ \\
\hline
\end{tabular}


Table 1-Continued

\begin{tabular}{|c|c|c|c|c|c|}
\hline SN Ia & $z_{c}$ & $m_{B}{ }^{1}$ & $s$ & $c$ & $\mu^{2}$ \\
\hline sn02hw & $0.016(0.001)$ & $16.626(0.139)$ & $0.748(0.009)$ & $0.459(0.008)$ & $34.590(0.221)$ \\
\hline sn02jy & $0.020(0.001)$ & $15.743(0.138)$ & $1.080(0.009)$ & $0.053(0.005)$ & $35.169(0.197)$ \\
\hline $\mathrm{sn} 02 \mathrm{kf}$ & $0.019(0.001)$ & $15.659(0.138)$ & $0.868(0.005)$ & $0.019(0.005)$ & $34.912(0.200)$ \\
\hline $\mathrm{sn} 03 \mathrm{U}$ & $0.028(0.001)$ & $16.481(0.138)$ & $0.762(0.008)$ & $0.072(0.007)$ & $35.463(0.173)$ \\
\hline sn03W & $0.021(0.001)$ & $15.865(0.138)$ & $0.932(0.001)$ & $0.189(0.004)$ & $34.757(0.192)$ \\
\hline sn03ch & $0.030(0.001)$ & $16.656(0.138)$ & $0.807(0.010)$ & $0.022(0.006)$ & $35.826(0.169)$ \\
\hline sn03cq & $0.034(0.001)$ & $17.208(0.140)$ & $0.898(0.011)$ & $0.202(0.016)$ & $36.024(0.169)$ \\
\hline sn03fa & $0.039(0.001)$ & $16.643(0.138)$ & $1.067(0.006)$ & $-0.008(0.004)$ & $36.212(0.157)$ \\
\hline sn03ic & $0.054(0.001)$ & $17.640(0.139)$ & $0.738(0.023)$ & $0.036(0.015)$ & $36.685(0.157)$ \\
\hline sn03it & $0.024(0.001)$ & $16.351(0.139)$ & $0.817(0.013)$ & $0.089(0.012)$ & $35.358(0.185)$ \\
\hline sn03iv & $0.034(0.001)$ & $16.951(0.138)$ & $0.715(0.016)$ & $-0.016(0.009)$ & $36.104(0.165)$ \\
\hline sn04as & $0.032(0.001)$ & $16.977(0.138)$ & $0.970(0.011)$ & $0.148(0.008)$ & $36.021(0.166)$ \\
\hline sn04bg & $0.022(0.001)$ & $15.618(0.141)$ & $1.038(0.011)$ & $0.015(0.012)$ & $35.091(0.193)$ \\
\hline $\mathrm{sn} 04 \mathrm{~L}$ & $0.033(0.001)$ & $17.307(0.142)$ & $0.890(0.018)$ & $0.216(0.016)$ & $36.074(0.172)$ \\
\hline sn05eq & $0.028(0.001)$ & $16.268(0.138)$ & $1.119(0.005)$ & $0.059(0.004)$ & $35.726(0.171)$ \\
\hline sn05eu & $0.034(0.001)$ & $16.281(0.138)$ & $1.008(0.018)$ & $-0.129(0.004)$ & $36.088(0.163)$ \\
\hline sn05hc & $0.045(0.001)$ & $17.373(0.138)$ & $0.977(0.007)$ & $0.068(0.005)$ & $36.634(0.153)$ \\
\hline sn05hj & $0.057(0.001)$ & $17.654(0.143)$ & $1.156(0.043)$ & $0.028(0.010)$ & $37.240(0.162)$ \\
\hline sn05iq & $0.033(0.001)$ & $16.775(0.138)$ & $0.833(0.009)$ & $-0.051(0.008)$ & $36.166(0.165)$ \\
\hline sn05ir & $0.075(0.001)$ & 18.411(0.139) & $1.281(0.065)$ & $0.044(0.018)$ & $38.109(0.172)$ \\
\hline sn05ki & $0.021(0.001)$ & $15.566(0.138)$ & $0.794(0.004)$ & $-0.034(0.006)$ & $34.864(0.194)$ \\
\hline sn05ls & $0.021(0.001)$ & $16.213(0.138)$ & $1.076(0.009)$ & $0.357(0.005)$ & $34.846(0.195)$ \\
\hline sn05lz & $0.040(0.001)$ & $17.596(0.138)$ & $0.807(0.009)$ & $0.150(0.006)$ & $36.432(0.157)$ \\
\hline $\mathrm{sn} 05 \mathrm{mc}$ & $0.026(0.001)$ & $17.184(0.138)$ & $0.622(0.006)$ & $0.256(0.005)$ & $35.518(0.176)$ \\
\hline $\mathrm{sn} 05 \mathrm{~ms}$ & $0.026(0.001)$ & $16.185(0.138)$ & $0.994(0.005)$ & $0.059(0.003)$ & $35.489(0.176)$ \\
\hline $\mathrm{sn} 05 \mathrm{mz}$ & $0.017(0.001)$ & $16.368(0.138)$ & $0.574(0.002)$ & $0.262(0.005)$ & $34.628(0.215)$ \\
\hline sn05na & $0.027(0.001)$ & $16.059(0.138)$ & $0.920(0.001)$ & $0.003(0.005)$ & $35.417(0.174)$ \\
\hline sn06ac & $0.024(0.001)$ & $16.147(0.138)$ & $0.869(0.005)$ & $0.092(0.004)$ & $35.210(0.183)$ \\
\hline sn06al & $0.069(0.001)$ & $18.379(0.138)$ & $0.775(0.003)$ & $-0.049(0.012)$ & $37.691(0.148)$ \\
\hline sn06an & $0.065(0.001)$ & $18.115(0.141)$ & $0.970(0.021)$ & $0.055(0.011)$ & $37.400(0.152)$ \\
\hline sn06ar & $0.023(0.001)$ & $16.442(0.138)$ & $0.835(0.009)$ & $0.153(0.006)$ & $35.305(0.186)$ \\
\hline sn06ax & $0.018(0.001)$ & $15.054(0.138)$ & $0.969(0.003)$ & $-0.017(0.003)$ & $34.524(0.208)$ \\
\hline sn06az & $0.032(0.001)$ & $16.441(0.138)$ & $0.818(0.003)$ & $-0.049(0.003)$ & $35.806(0.165)$ \\
\hline sn06bq & $0.021(0.001)$ & $16.273(0.138)$ & $0.773(0.011)$ & $0.156(0.001)$ & $35.053(0.191)$ \\
\hline sn06bt & $0.033(0.001)$ & $16.915(0.138)$ & $0.990(0.007)$ & $0.149(0.004)$ & $35.981(0.164)$ \\
\hline sn06bu & $0.084(0.001)$ & $18.086(0.160)$ & $1.400(0.044)$ & $-0.037(0.031)$ & $38.143(0.190)$ \\
\hline sn06bz & $0.028(0.001)$ & $18.244(0.138)$ & $0.496(0.005)$ & $0.559(0.009)$ & $35.638(0.174)$ \\
\hline $\mathrm{sn} 06 \mathrm{cc}$ & $0.033(0.001)$ & $17.766(0.138)$ & $0.946(0.005)$ & $0.369(0.004)$ & $36.209(0.163)$ \\
\hline $\operatorname{sn} 06 \mathrm{cf}$ & $0.042(0.001)$ & $17.082(0.138)$ & $0.887(0.001)$ & $-0.011(0.007)$ & $36.436(0.155)$ \\
\hline $\operatorname{sn} 06 \mathrm{cj}$ & $0.068(0.001)$ & $18.145(0.138)$ & $1.105(0.053)$ & $-0.017(0.010)$ & $37.784(0.161)$ \\
\hline sn06cp & $0.023(0.001)$ & $15.977(0.138)$ & $0.941(0.005)$ & $0.151(0.005)$ & $34.977(0.184)$ \\
\hline sn06cq & $0.049(0.001)$ & $17.541(0.138)$ & $0.990(0.043)$ & $0.033(0.009)$ & $36.907(0.161)$ \\
\hline sn06ej & $0.019(0.001)$ & $15.706(0.138)$ & $0.819(0.006)$ & $-0.006(0.007)$ & $34.962(0.202)$ \\
\hline sn06en & $0.031(0.001)$ & $16.791(0.138)$ & $0.911(0.013)$ & $0.074(0.000)$ & $35.953(0.167)$ \\
\hline sn06et & $0.021(0.001)$ & $15.937(0.139)$ & $1.106(0.014)$ & $0.156(0.010)$ & $35.128(0.193)$ \\
\hline sn06gj & $0.028(0.001)$ & $17.600(0.139)$ & $0.680(0.011)$ & $0.316(0.011)$ & $35.849(0.175)$ \\
\hline
\end{tabular}


Table 1-Continued

\begin{tabular}{|c|c|c|c|c|c|}
\hline SN Ia & $z_{C M B}$ & $m_{B}^{1}$ & $s$ & $c$ & $\mu^{2}$ \\
\hline sn06gr & $0.034(0.001)$ & $16.933(0.138)$ & $0.991(0.005)$ & $0.140(0.004)$ & $36.023(0.162)$ \\
\hline sn06kf & $0.021(0.001)$ & $15.767(0.138)$ & $0.687(0.006)$ & $-0.061(0.007)$ & $35.001(0.194)$ \\
\hline sn06le & $0.017(0.001)$ & $14.757(0.138)$ & $1.021(0.003)$ & $-0.039(0.003)$ & $34.348(0.212)$ \\
\hline sn06mo & $0.036(0.001)$ & $17.357(0.138)$ & $0.696(0.000)$ & $0.053(0.008)$ & $36.306(0.160)$ \\
\hline sn06nz & $0.037(0.001)$ & $18.032(0.139)$ & $0.596(0.001)$ & $0.281(0.013)$ & $36.267(0.162)$ \\
\hline sn06oa & $0.059(0.001)$ & $17.819(0.138)$ & $0.924(0.013)$ & $0.064(0.007)$ & $37.025(0.148)$ \\
\hline sn06ob & $0.058(0.001)$ & $18.185(0.138)$ & $0.699(0.011)$ & $0.050(0.007)$ & $37.146(0.149)$ \\
\hline sn06on & $0.069(0.001)$ & $18.398(0.141)$ & $1.056(0.014)$ & $0.132(0.027)$ & $37.591(0.163)$ \\
\hline sn06os & $0.032(0.001)$ & $17.636(0.139)$ & $0.892(0.018)$ & $0.433(0.013)$ & $35.844(0.169)$ \\
\hline sn06qo & $0.030(0.001)$ & $16.790(0.138)$ & $0.984(0.005)$ & $0.188(0.006)$ & $35.746(0.168)$ \\
\hline sn06S & $0.033(0.001)$ & $16.831(0.138)$ & $1.035(0.020)$ & $0.117(0.001)$ & $36.036(0.165)$ \\
\hline sn06sr & $0.023(0.001)$ & $16.118(0.138)$ & $0.835(0.009)$ & $0.041(0.006)$ & $35.272(0.185)$ \\
\hline sn06td & $0.015(0.001)$ & $15.718(0.138)$ & $0.776(0.006)$ & $0.142(0.005)$ & $34.535(0.231)$ \\
\hline sn06te & $0.032(0.001)$ & $16.502(0.138)$ & $0.916(0.004)$ & $-0.052(0.009)$ & $35.996(0.166)$ \\
\hline sn07ae & $0.064(0.001)$ & $17.657(0.147)$ & $1.105(0.023)$ & $0.007(0.018)$ & $37.233(0.162)$ \\
\hline sn07ai & $0.032(0.001)$ & $16.856(0.139)$ & $1.099(0.001)$ & $0.168(0.011)$ & $36.008(0.167)$ \\
\hline sn07au & $0.020(0.001)$ & $16.474(0.138)$ & $0.630(0.003)$ & $0.217(0.005)$ & $34.916(0.197)$ \\
\hline sn07bc & $0.022(0.001)$ & $15.870(0.138)$ & $0.814(0.007)$ & $0.039(0.008)$ & $35.003(0.190)$ \\
\hline sn07bd & $0.032(0.001)$ & $16.520(0.138)$ & $0.791(0.003)$ & $-0.004(0.004)$ & $35.736(0.164)$ \\
\hline sn07ca & $0.015(0.001)$ & $15.907(0.138)$ & $0.986(0.005)$ & $0.258(0.005)$ & $34.687(0.229)$ \\
\hline $\mathrm{sn} 07 \mathrm{ci}$ & $0.019(0.001)$ & $15.886(0.138)$ & $0.693(0.003)$ & $0.121(0.005)$ & $34.656(0.202)$ \\
\hline sn07co & $0.027(0.001)$ & $16.521(0.138)$ & $0.920(0.003)$ & $0.182(0.003)$ & $35.415(0.174)$ \\
\hline $\mathrm{sn} 07 \mathrm{cq}$ & $0.025(0.001)$ & $15.786(0.138)$ & $0.883(0.009)$ & $0.006(0.005)$ & $35.090(0.180)$ \\
\hline $\mathrm{sn} 07 \mathrm{~F}$ & $0.024(0.001)$ & $15.864(0.138)$ & $1.014(0.003)$ & $-0.009(0.004)$ & $35.370(0.181)$ \\
\hline $\mathrm{sn} 07 \mathrm{O}$ & $0.036(0.001)$ & $16.676(0.138)$ & $0.914(0.001)$ & $-0.052(0.008)$ & $36.170(0.162)$ \\
\hline sn07qe & $0.023(0.001)$ & $16.026(0.138)$ & $1.002(0.003)$ & $0.084(0.003)$ & $35.275(0.180)$ \\
\hline $\mathrm{sn} 07 \mathrm{R}$ & $0.031(0.001)$ & $16.559(0.141)$ & $0.820(0.009)$ & $-0.111(0.015)$ & $36.087(0.175)$ \\
\hline sn08L & $0.019(0.001)$ & $15.071(0.138)$ & $0.775(0.009)$ & $-0.077(0.004)$ & $34.457(0.204)$ \\
\hline sn08bf & $0.026(0.001)$ & $15.716(0.138)$ & $1.038(0.006)$ & $0.019(0.005)$ & $35.177(0.178)$ \\
\hline
\end{tabular}

${ }^{1}$ The uncertainty of $m_{B}$ is a modified value for the Union set SN Ia, calculated by subtracting the color and stretch uncertainties from $\sigma_{\mu}$ of $\mathrm{K} 08$ so as to include the sample-dependent systematic uncertainty of K08. For the CfA3 sample, 0.138 mag is added in quadrature to the actual uncertainty of $m_{B}$.

${ }^{2}$ Distance moduli were calculated with $\mathrm{H}_{\mathrm{o}}=65 \mathrm{kms}^{-1}, M_{B}=-19.46, \alpha=1.34_{-0.08}^{+0.08}$ and $\beta=2.59_{-0.08}^{+0.12}$. Uncertainty of $\mu$ includes uncertainties of $m_{B}, s, c, \sigma_{v-p e c}\left(400 \mathrm{~km} \mathrm{~s}^{-1}\right)$, and $\sigma_{z}$. 
Table 2. SALT2 Light Curve Fit Parameters and Distances.

\begin{tabular}{|c|c|c|c|c|c|}
\hline SN Ia & $z_{C M B}$ & $m_{B}$ & $x_{1}$ & $c$ & $\mu^{1}$ \\
\hline 03D1au & $0.504(0.001)$ & $22.990(0.013)$ & $1.058(0.209)$ & $0.018(0.022)$ & $42.498(0.169)$ \\
\hline 03D1aw & $0.582(0.001)$ & $23.557(0.018)$ & $-0.039(0.240)$ & $0.055(0.031)$ & $0.178)$ \\
\hline 03D1ax & $0.496(0.001)$ & $22.956(0.015)$ & $-0.931(0.167)$ & $-0.033(0.026)$ & $42.384(0.172)$ \\
\hline 03D1bp & $0.346(0.001)$ & $22.412(0.036)$ & $-1.153(0.224)$ & $0.081(0.039)$ & $41.534(0.190)$ \\
\hline 03D1co & $0.679(0.001)$ & $24.102(0.046)$ & $0.666(0.587)$ & $-0.008(0.055)$ & $43.632(0.222)$ \\
\hline 03D1ew & $0.868(0.001)$ & $24.361(0.056)$ & $0.297(0.411)$ & $-0.072(0.180)$ & $44.012(0.479)$ \\
\hline 03D1fc & $0.331(0.001)$ & $21.813(0.020)$ & $0.218(0.186)$ & $0.024(0.017)$ & $41.220(0.166)$ \\
\hline 03D1fl & $0.688(0.001)$ & $23.599(0.017)$ & $-0.262(0.255)$ & $-0.070(0.028)$ & $43.187(0.176)$ \\
\hline 03D1fq & $0.800(0.001)$ & $24.529(0$ & $-1.622(0.525)$ & -0.034 & 43.888 \\
\hline 03D1gt & $0.548(0.001)$ & $24.043(0.048)$ & $-2.211(0.425)$ & $0.298(0.062)$ & $42.517(0.230)$ \\
\hline 03D3af & $0.532(0.001)$ & $23.486(0.027)$ & $-0.298(0.2$ & $0.027(0$. & 42.830 \\
\hline 03D3aw & $0.449(0.001)$ & $22.548(0.038)$ & $-0.132(0.291)$ & $-0.080(0.035)$ & $42.175(0.186)$ \\
\hline 03D3ay & $0.371(0.001)$ & $22.215(0$ & $0.250(0.280)$ & $-0.051(0$. & $41.811(0.185)$ \\
\hline 03D3ba & $0.291(0.001)$ & $21.982(0.029)$ & $0.364(0.172)$ & $0.207(0.029)$ & \\
\hline 03D3cc & $0.463(0.001)$ & $22.660(0.046)$ & $0.417(0.294)$ & $-0.009(0$. & $42.168(0.198)$ \\
\hline 03D3cd & $0.461(0.001)$ & $22.593(0.070)$ & $2.016(1.001)$ & $0.017(0.048)$ & 42.202 \\
\hline 03D4ag & & & & -0 . & \\
\hline 03D4at & 0.63 & 23.71 & -0. & -0.0 & \\
\hline $03 \mathrm{D} 4 \mathrm{cx}$ & $0.949(0.001)$ & $24.484(0.069)$ & $-0.586(0.5$ & 0.120 & 43.56 \\
\hline 03D4cy & 0.927 & 7) & 0.258 & -0.366 & 45. \\
\hline $03 \mathrm{D} 4 \mathrm{cz}$ & 0.695( & 24.039 & $-2.360(C$ & -0.069 & 43.40 \\
\hline 03D4dh & 0.62 & 23.367 & 0.845( & -0.0 & 42.928 \\
\hline 03D4di & $0.905(0.001)$ & $24.271(0.055)$ & 1.029 & -0.02 & \\
\hline 03D4dy & $0.604(0.001)$ & $23.288(0.018)$ & $0.887(0.195)$ & $0.079(0.027)$ & $42.628(0.173)$ \\
\hline 03D4fd & $0.791(0.001)$ & $24.216(0.025)$ & $-0.143(0.479)$ & $-0.043(0.055)$ & $43.749(0.216)$ \\
\hline 03D4gf & 0.5 & 23 & 0.2 & -0.0 & 75) \\
\hline 03D4gg & 0.592 & & & & 42. \\
\hline 03D4gl & $0.571(0.001)$ & $23.361(0.058)$ & $3.871(0.884)$ & $0.038(0.073)$ & $43.111(0.263)$ \\
\hline 04D1ag & $0.557(0.001)$ & $23.025(0.011)$ & $0.414(0.1$ & $-0.116(0.021)$ & $42.798(0.168)$ \\
\hline 04D1aj & 0.721 & 23.860 & 0.525 & 1) & $43.337(0.196)$ \\
\hline 04D1ak & $0.526(0.001)$ & $23.612(0.041)$ & $-2.035(0.353)$ & $0.052(0.052)$ & $42.714(0.211)$ \\
\hline 04D2cf & $0.369(0.001)$ & $22.559(0.035)$ & $-1.439(0.315)$ & $0.052(0.032)$ & $41.722(0.183)$ \\
\hline 04D2fp & $0.415(0.001)$ & $22.521(0.022)$ & $0.130(0.198)$ & $-0.029(0.026)$ & $42.048(0.173)$ \\
\hline 04D2fs & $0.357(0.001)$ & $22.395(0.0$ & $-0.091(0.154)$ & $0.093(0.017)$ & $41.598(0.165)$ \\
\hline 04D2gb & $0.430(0.001)$ & 22.809 & -2.313( & 0.008 & $41.991(0.203)$ \\
\hline 04D2gp & $0.707(0.001)$ & $24.149(0.048)$ & $-1.868(0.529)$ & $-0.047(0.074)$ & $43.515(0.254)$ \\
\hline 04D2iu & $0.691(0.001)$ & $24.266(0.046)$ & $-1.907(0.538)$ & $0.091(0.070)$ & $43.285(0.245)$ \\
\hline 04D2ja & $0.741(0.001)$ & $24.109(0.043)$ & $0.003(0.433)$ & $-0.133(0.054)$ & $43.881(0.216)$ \\
\hline 04D3co & $0.620(0.001)$ & $23.771(0.029)$ & $-0.794(0.276)$ & $-0.070(0.041)$ & $43.304(0.192)$ \\
\hline 04D3cp & $0.830(0.001)$ & $24.198(0.043)$ & $1.317(0.403)$ & $-0.431(0.190)$ & $44.844(0.500)$ \\
\hline 04D3cy & $0.643(0.001)$ & $23.795(0.027)$ & $0.216(0.283)$ & $0.017(0.036)$ & $43.217(0.186)$ \\
\hline 04D3dd & $1.010(0.001)$ & $25.063(0.159)$ & $1.138(0.655)$ & $-0.121(0.214)$ & $44.924(0.580)$ \\
\hline 04D3df & $0.470(0.001)$ & $23.513(0.029)$ & $-2.280(0.298)$ & $0.142(0.038)$ & $42.367(0.189)$ \\
\hline 04D3do & $0.610(0.001)$ & $23.564(0.018)$ & $-1.280(0.220)$ & $-0.064(0.030)$ & $43.032(0.177)$ \\
\hline 04D3ez & $0.263(0.001)$ & $21.662(0.017)$ & $-0.976(0.144)$ & $0.084(0.016)$ & $40.794(0.165)$ \\
\hline 04D3fk & $0.358(0.001)$ & $22.525(0.017)$ & $-0.412(0.128)$ & $0.137(0.016)$ & $41.587(0.165)$ \\
\hline
\end{tabular}


Table 2-Continued

\begin{tabular}{|c|c|c|c|c|c|}
\hline SN Ia & $z_{C M B}$ & $m_{B}$ & $x_{1}$ & $c$ & $\mu^{1}$ \\
\hline 04D3fq & $0.730(0.001)$ & $24.092(0.026)$ & $-0.770(0.398)$ & $0.004(0.048)$ & $43.444(0.204)$ \\
\hline 04D3gt & $0.451(0.001)$ & $23.235(0.016)$ & $-0.226(0.164)$ & $0.297(0.023)$ & $41.919(0.169)$ \\
\hline 04D3gx & $0.910(0.001)$ & $24.716(0.074)$ & $-0.181(0.511)$ & $-0.227(0.176)$ & $44.702(0.472)$ \\
\hline 04D3hn & $0.552(0.001)$ & $23.472(0.015)$ & $-0.946(0.167)$ & $0.156(0.024)$ & $42.430(0.171)$ \\
\hline 04D3is & $0.710(0.001)$ & $24.229(0.031)$ & $0.205(0.405)$ & $0.203(0.046)$ & $43.188(0.202)$ \\
\hline 04D3ki & $0.930(0.001)$ & $24.860(0.100)$ & $-0.938(0.457)$ & $-0.226(0.207)$ & $44.763(0.549)$ \\
\hline 04D3kr & $0.337(0.001)$ & $21.932(0.021)$ & $1.097(0.185)$ & $0.013(0.017)$ & $41.456(0.166)$ \\
\hline 04D3ks & $0.752(0.001)$ & $23.851(0.037)$ & $0.332(0.433)$ & $-0.059(0.053)$ & $43.475(0.214)$ \\
\hline 04D3lu & $0.822(0.001)$ & $24.339(0.031)$ & $-0.082(0.354)$ & $0.096(0.125)$ & $43.534(0.350)$ \\
\hline 04D3ml & $0.950(0.001)$ & $24.494(0.070)$ & $1.356(0.641)$ & $0.096(0.141)$ & $43.841(0.395)$ \\
\hline 04D3nc & $0.817(0.001)$ & $24.272(0.056)$ & $1.812(1.234)$ & $0.007(0.154)$ & $43.885(0.437)$ \\
\hline 04D3nh & $0.340(0.001)$ & $22.089(0.016)$ & $0.418(0.171)$ & $0.033(0.015)$ & $41.494(0.164)$ \\
\hline 04D3nr & $0.960(0.001)$ & $24.528(0.061)$ & $-0.585(0.536)$ & $0.083(0.116)$ & $43.705(0.337)$ \\
\hline 04D3ny & $0.810(0.001)$ & $24.277(0.038)$ & $0.852(0.889)$ & $-0.076(0.170)$ & $43.996(0.461)$ \\
\hline 04D3oe & $0.756(0.001)$ & $24.070(0.031)$ & $-1.849(0.459)$ & $-0.282(0.051)$ & $44.019(0.211)$ \\
\hline 04D4an & $0.613(0.001)$ & $24.027(0.027)$ & $-1.601(0.387)$ & $0.078(0.035)$ & $43.111(0.187)$ \\
\hline 04D4bk & $0.840(0.001)$ & $24.291(0.033)$ & $1.192(0.650)$ & $0.209(0.103)$ & $43.339(0.309)$ \\
\hline 04D4bq & $0.550(0.001)$ & $23.333(0.024)$ & $0.109(0.404)$ & $0.126(0.030)$ & $42.475(0.182)$ \\
\hline $04 \mathrm{D} 4 \mathrm{dm}$ & $0.811(0.001)$ & $24.398(0.040)$ & $0.859(1.095)$ & $-0.130(0.165)$ & $44.250(0.455)$ \\
\hline $2002 \mathrm{dc}$ & $0.475(0.010)$ & $23.175(0.129)$ & $-1.233(0.346)$ & $0.077(0.126)$ & $42.299(0.375)$ \\
\hline $2002 \mathrm{dd}$ & $0.950(0.010)$ & $24.596(0.033)$ & $1.352(0.373)$ & $0.104(0.046)$ & $43.921(0.201)$ \\
\hline Eagle & $1.020(0.010)$ & $24.931(0.027)$ & $-0.178(0.406)$ & $0.004(0.050)$ & $44.345(0.206)$ \\
\hline Ferguson & $1.020(0.010)$ & $24.816(0.060)$ & $0.956(0.568)$ & $0.099(0.059)$ & $44.112(0.230)$ \\
\hline Gabi & $1.120(0.010)$ & $25.080(0.024)$ & $0.091(0.372)$ & $0.009(0.042)$ & $44.509(0.195)$ \\
\hline Koekemoer & $1.230(0.010)$ & $25.284(0.092)$ & $-0.022(0.651)$ & $-0.034(0.074)$ & $44.809(0.268)$ \\
\hline Lancaster & $1.230(0.010)$ & $26.057(0.050)$ & $-0.626(0.678)$ & $0.130(0.045)$ & $45.113(0.211)$ \\
\hline Manipogo & $0.854(0.010)$ & $24.535(0.028)$ & $-1.197(0.425)$ & $0.016(0.048)$ & $43.813(0.205)$ \\
\hline Mcguire & $1.370(0.010)$ & $25.616(0.046)$ & $0.750(0.626)$ & $0.023(0.036)$ & $45.079(0.199)$ \\
\hline Ombo & $0.975(0.010)$ & $24.858(0.031)$ & $1.850(0.426)$ & $0.069(0.034)$ & $44.323(0.187)$ \\
\hline Patuxent & $0.970(0.010)$ & $24.983(0.025)$ & $-0.866(1.230)$ & $-0.122(0.061)$ & $44.639(0.255)$ \\
\hline Rakke & $0.740(0.010)$ & $23.902(0.043)$ & $-0.280(0.330)$ & $-0.085(0.040)$ & $43.526(0.194)$ \\
\hline Spock & $0.839(0.010)$ & $24.133(0.132)$ & $-0.124(0.508)$ & $-0.018(0.088)$ & $43.608(0.305)$ \\
\hline Strolger & $1.010(0.010)$ & $24.945(0.102)$ & $2.255(1.978)$ & $-0.071(0.061)$ & $44.797(0.317)$ \\
\hline Thames & $0.954(0.010)$ & $24.638(0.258)$ & $-0.688(0.631)$ & $-0.056(0.118)$ & $44.149(0.426)$ \\
\hline Zwicky & $0.521(0.010)$ & $23.055(0.055)$ & $1.129(0.651)$ & $0.045(0.042)$ & $42.504(0.208)$ \\
\hline anguta & $0.670(0.010)$ & $24.228(0.035)$ & $-1.181(0.328)$ & $0.087(0.032)$ & $43.331(0.184)$ \\
\hline aphrodite & $1.300(0.010)$ & $25.623(0.024)$ & $0.518(0.371)$ & $0.033(0.038)$ & $45.038(0.189)$ \\
\hline athena & $1.400(0.010)$ & $26.549(0.184)$ & $-0.496(2.243)$ & $0.000(0.000)$ & $45.940(0.336)$ \\
\hline bilbo & $0.216(0.010)$ & $22.233(0.186)$ & $-0.021(0.716)$ & $0.485(0.116)$ & $40.473(0.385)$ \\
\hline borg & $1.340(0.010)$ & $25.699(0.042)$ & $1.716(0.637)$ & $0.111(0.047)$ & $45.046(0.212)$ \\
\hline elvis & $0.840(0.010)$ & $24.359(0.020)$ & $-0.303(0.463)$ & $0.085(0.027)$ & $43.558(0.179)$ \\
\hline frodo & $0.735(0.010)$ & $23.981(0.071)$ & $-0.434(0.334)$ & $-0.015(0.071)$ & $43.415(0.249)$ \\
\hline gilgamesh & $1.551(0.010)$ & $26.513(0.055)$ & $-0.017(0.848)$ & $0.252(0.037)$ & $45.329(0.211)$ \\
\hline inanna & $1.307(0.010)$ & $26.631(0.123)$ & $-2.938(1.896)$ & $0.209(0.085)$ & $45.249(0.351)$ \\
\hline isis & $0.526(0.010)$ & $24.229(0.100)$ & $2.344(1.088)$ & $0.191(0.054)$ & $43.441(0.257)$ \\
\hline mcenroe & $0.900(0.010)$ & $24.084(0.015)$ & $-0.132(0.227)$ & $0.137(0.028)$ & $43.174(0.175)$ \\
\hline
\end{tabular}


Table 2-Continued

\begin{tabular}{|c|c|c|c|c|c|}
\hline SN Ia & $z_{C M B}$ & $m_{B}$ & $x_{1}$ & $c$ & $\mu^{1}$ \\
\hline nanna & $1.140(0.010)$ & $25.385(0.041)$ & $0.232(0.431)$ & $0.052(0.063)$ & $44.722(0.231)$ \\
\hline qiqirn & $0.640(0.010)$ & $24.271(0.206)$ & $-0.999(0.940)$ & $0.212(0.122)$ & $43.085(0.410)$ \\
\hline ramone & $0.954(0.010)$ & $24.581(0.064)$ & $-1.193(0.556)$ & $-0.167(0.060)$ & $44.312(0.233)$ \\
\hline sasquatch & $1.390(0.010)$ & $25.857(0.064)$ & $1.625(0.868)$ & $0.190(0.047)$ & $44.997(0.225)$ \\
\hline torngasak & $1.265(0.010)$ & $25.715(0.047)$ & $0.260(0.705)$ & $0.127(0.047)$ & $44.870(0.214)$ \\
\hline vilas & $0.935(0.010)$ & $24.391(0.037)$ & $-0.217(0.262)$ & $0.071(0.052)$ & $43.635(0.209)$ \\
\hline b010 & $0.590(0.003)$ & $23.405(0.079)$ & $1.999(0.909)$ & $-0.117(0.095)$ & $43.346(0.309)$ \\
\hline b013 & $0.427(0.003)$ & $22.623(0.043)$ & $-0.596(0.405)$ & $0.024(0.052)$ & $41.943(0.213)$ \\
\hline b020 & $0.426(0.003)$ & $22.461(0.218)$ & $-1.417(1.830)$ & $0.043(0.199)$ & $41.648(0.592)$ \\
\hline d033 & $0.530(0.008)$ & $23.088(0.081)$ & $3.825(1.148)$ & $-0.291(0.099)$ & $43.649(0.325)$ \\
\hline d083 & $0.333(0.001)$ & $21.002(0.033)$ & $1.250(0.220)$ & $-0.053(0.035)$ & $40.705(0.185)$ \\
\hline d084 & $0.516(0.006)$ & $23.647(0.065)$ & $0.615(0.886)$ & $-0.024(0.100)$ & $43.213(0.314)$ \\
\hline d085 & $0.401(0.008)$ & $22.450(0.048)$ & $0.654(0.390)$ & $0.043(0.059)$ & $41.854(0.224)$ \\
\hline d086 & $0.204(0.003)$ & $21.098(0.093)$ & $-0.303(0.196)$ & $0.173(0.082)$ & $40.080(0.275)$ \\
\hline d087 & $0.337(0.004)$ & $21.965(0.058)$ & $-0.711(0.337)$ & $0.015(0.062)$ & $41.295(0.231)$ \\
\hline d089 & $0.424(0.003)$ & $22.460(0.041)$ & $0.123(0.382)$ & $-0.052(0.049)$ & $42.044(0.207)$ \\
\hline d093 & $0.361(0.003)$ & $21.897(0.048)$ & $1.087(0.279)$ & $-0.102(0.048)$ & $41.705(0.205)$ \\
\hline d097 & $0.434(0.007)$ & $22.501(0.049)$ & $1.744(0.504)$ & $0.021(0.058)$ & $42.073(0.226)$ \\
\hline d099 & $0.210(0.002)$ & $21.126(0.102)$ & $-0.758(0.214)$ & $-0.047(0.088)$ & $40.606(0.289)$ \\
\hline $\mathrm{d} 117$ & $0.301(0.005)$ & $22.367(0.100)$ & $-1.845(0.358)$ & $0.097(0.092)$ & $41.378(0.296)$ \\
\hline d149 & $0.344(0.008)$ & $22.092(0.045)$ & $0.385(0.355)$ & $0.007(0.044)$ & $41.556(0.200)$ \\
\hline $\mathrm{e} 020$ & $0.159(0.006)$ & $21.062(0.150)$ & $0.029(0.377)$ & $0.372(0.127)$ & $39.585(0.384)$ \\
\hline $\mathrm{e} 029$ & $0.332(0.008)$ & $22.390(0.078)$ & $-2.800(0.947)$ & $0.040(0.079)$ & $41.442(0.282)$ \\
\hline e108 & $0.472(0.007)$ & $22.622(0.088)$ & $3.370(1.706)$ & $-0.069(0.092)$ & $42.586(0.341)$ \\
\hline e132 & $0.235(0.006)$ & $21.771(0.065)$ & $-0.337(0.248)$ & $0.300(0.060)$ & $40.435(0.229)$ \\
\hline e136 & $0.348(0.006)$ & $22.735(0.054)$ & $-2.029(0.611)$ & $0.134(0.060)$ & $41.636(0.233)$ \\
\hline e138 & $0.611(0.006)$ & $23.748(0.050)$ & $-3.866(0.730)$ & $0.259(0.101)$ & $42.147(0.310)$ \\
\hline e140 & $0.614(0.006)$ & $23.345(0.024)$ & $0.676(0.475)$ & $0.044(0.046)$ & $42.749(0.203)$ \\
\hline e147 & $0.641(0.008)$ & $23.336(0.047)$ & $-0.385(0.442)$ & $-0.105(0.058)$ & $42.999(0.223)$ \\
\hline e148 & $0.429(0.006)$ & $22.633(0.034)$ & $-1.105(0.350)$ & $-0.049(0.045)$ & $42.081(0.199)$ \\
\hline e149 & $0.491(0.006)$ & $23.686(0.054)$ & $5.000(0.253)$ & $0.581(0.076)$ & $42.209(0.253)$ \\
\hline f011 & $0.536(0.006)$ & $23.279(0.037)$ & $-1.458(0.500)$ & $0.060(0.062)$ & $42.422(0.230)$ \\
\hline f041 & $0.558(0.007)$ & $23.120(0.044)$ & $2.736(1.100)$ & $-0.092(0.065)$ & $43.075(0.257)$ \\
\hline f076 & $0.410(0.004)$ & $22.346(0.064)$ & $-1.000(0.638)$ & $0.060(0.090)$ & $41.536(0.288)$ \\
\hline $\mathrm{f} 216$ & $0.595(0.011)$ & $23.774(0.077)$ & $-3.248(1.067)$ & $-0.038(0.132)$ & $42.973(0.387)$ \\
\hline $\mathrm{f} 221$ & $0.443(0.004)$ & $23.080(0.088)$ & $-1.756(0.685)$ & $-0.363(0.117)$ & $43.239(0.350)$ \\
\hline $\mathrm{f} 231$ & $0.620(0.008)$ & $23.447(0.035)$ & $1.243(0.354)$ & $-0.076(0.062)$ & $43.205(0.227)$ \\
\hline $\mathrm{f} 235$ & $0.422(0.006)$ & $22.403(0.056)$ & $-1.202(0.494)$ & $-0.079(0.065)$ & $41.917(0.238)$ \\
\hline f244 & $0.546(0.005)$ & $23.311(0.050)$ & $-1.016(0.793)$ & $0.003(0.080)$ & $42.641(0.271)$ \\
\hline g001 & $0.268(0.002)$ & $21.441(0.078)$ & $-2.030(0.312)$ & $-0.066(0.070)$ & $40.834(0.249)$ \\
\hline g005 & $0.218(0.007)$ & $21.404(0.089)$ & $1.348(0.306)$ & $0.319(0.081)$ & $40.197(0.272)$ \\
\hline g052 & $0.384(0.006)$ & $22.335(0.082)$ & $-2.037(0.419)$ & $-0.115(0.090)$ & $41.851(0.289)$ \\
\hline g055 & $0.302(0.006)$ & $23.119(0.126)$ & $2.087(0.589)$ & $0.489(0.109)$ & $41.568(0.342)$ \\
\hline g097 & $0.339(0.004)$ & $22.267(0.078)$ & $0.481(0.426)$ & $0.209(0.073)$ & $41.241(0.257)$ \\
\hline g160 & $0.507(0.019)$ & $22.972(0.070)$ & $1.439(0.600)$ & $0.117(0.079)$ & $42.276(0.269)$ \\
\hline g240 & $0.689(0.005)$ & $23.410(0.075)$ & $-0.046(0.883)$ & $-0.130(0.084)$ & $43.170(0.286)$ \\
\hline
\end{tabular}


Table 2-Continued

\begin{tabular}{|c|c|c|c|c|c|}
\hline SN Ia & $z_{C M B}$ & $m_{B}$ & $x_{1}$ & $c$ & $\mu^{1}$ \\
\hline g276 & $0.244(0.001)$ & $21.701(0.059)$ & $-0.383(0.135)$ & $0.245(0.058)$ & $40.497(0.222)$ \\
\hline h300 & $0.656(0.011)$ & $23.492(0.053)$ & $0.737(0.648)$ & $0.007(0.066)$ & $42.994(0.243)$ \\
\hline h319 & $0.478(0.002)$ & $22.882(0.045)$ & $1.025(0.664)$ & $0.011(0.052)$ & $42.403(0.220)$ \\
\hline h323 & $0.603(0.007)$ & $23.481(0.045)$ & $-0.314(0.584)$ & $0.028(0.077)$ & $42.822(0.258)$ \\
\hline h342 & $0.421(0.005)$ & $22.407(0.061)$ & $1.359(0.658)$ & $-0.136(0.062)$ & $42.328(0.238)$ \\
\hline h359 & $0.348(0.005)$ & $22.597(0.051)$ & $-0.115(0.342)$ & $0.107(0.054)$ & $41.762(0.216)$ \\
\hline h363 & $0.211(0.005)$ & $22.054(0.097)$ & $-0.727(0.269)$ & $0.457(0.086)$ & $40.289(0.284)$ \\
\hline h364 & $0.344(0.004)$ & $21.716(0.045)$ & $-0.470(0.280)$ & $-0.099(0.048)$ & $41.354(0.205)$ \\
\hline $\mathrm{k} 411$ & $0.565(0.003)$ & $22.852(0.208)$ & $-0.630(1.395)$ & $-0.133(0.187)$ & $42.557(0.550)$ \\
\hline $\mathrm{k} 425$ & $0.274(0.003)$ & $22.018(0.070)$ & $-0.079(0.306)$ & $0.224(0.063)$ & $40.899(0.235)$ \\
\hline $\mathrm{k} 429$ & $0.171(0.006)$ & $20.359(0.112)$ & $0.150(0.194)$ & $-0.015(0.098)$ & $39.854(0.311)$ \\
\hline $\mathrm{k} 430$ & $0.576(0.007)$ & $23.801(0.040)$ & $-0.353(0.821)$ & $-0.062(0.088)$ & $43.359(0.286)$ \\
\hline $\mathrm{k} 441$ & $0.671(0.007)$ & $23.690(0.037)$ & $1.456(0.849)$ & $0.098(0.070)$ & $43.042(0.254)$ \\
\hline $\mathrm{k} 448$ & $0.405(0.007)$ & $23.326(0.113)$ & $0.278(1.361)$ & $0.282(0.124)$ & $42.099(0.391)$ \\
\hline k490 & $0.709(0.001)$ & $23.882(0.045)$ & $0.131(0.919)$ & $0.183(0.075)$ & $42.886(0.265)$ \\
\hline $\mathrm{m} 026$ & $0.656(0.006)$ & $23.604(0.065)$ & $-0.364(1.183)$ & $0.123(0.110)$ & $42.704(0.344)$ \\
\hline $\mathrm{m} 027$ & $0.285(0.003)$ & $22.478(0.105)$ & $0.536(0.853)$ & $0.244(0.089)$ & $41.371(0.305)$ \\
\hline $\mathrm{m} 032$ & $0.155(0.004)$ & $20.540(0.331)$ & $1.714(1.857)$ & $0.159(0.270)$ & $39.768(0.786)$ \\
\hline $\mathrm{m} 034$ & $0.562(0.006)$ & $23.351(0.071)$ & $0.383(0.976)$ & $-0.144(0.107)$ & $43.191(0.333)$ \\
\hline m039 & $0.249(0.003)$ & $22.220(0.110)$ & $-1.141(0.558)$ & $0.324(0.099)$ & $40.742(0.317)$ \\
\hline $\mathrm{m} 062$ & $0.316(0.004)$ & $21.887(0.091)$ & $-0.564(0.723)$ & $-0.066(0.077)$ & $41.433(0.275)$ \\
\hline $\mathrm{m} 158$ & $0.461(0.007)$ & $23.122(0.066)$ & $0.547(0.549)$ & $0.208(0.082)$ & $42.106(0.271)$ \\
\hline m193 & $0.336(0.006)$ & $21.607(0.074)$ & $-0.424(0.349)$ & $-0.063(0.056)$ & $41.161(0.226)$ \\
\hline n256 & $0.631(0.011)$ & $23.357(0.027)$ & $0.357(0.543)$ & $-0.085(0.044)$ & $43.047(0.201)$ \\
\hline n263 & $0.368(0.007)$ & $21.974(0.036)$ & $-0.581(0.324)$ & $-0.136(0.043)$ & $41.691(0.197)$ \\
\hline $\mathrm{n} 278$ & $0.308(0.006)$ & $21.742(0.057)$ & $-1.968(0.521)$ & $-0.036(0.059)$ & $41.068(0.229)$ \\
\hline n285 & $0.531(0.009)$ & $23.275(0.104)$ & $1.108(1.631)$ & $0.126(0.111)$ & $42.520(0.374)$ \\
\hline n326 & $0.267(0.006)$ & $21.956(0.161)$ & $-4.612(0.737)$ & $-0.049(0.146)$ & $41.041(0.433)$ \\
\hline n404 & $0.285(0.006)$ & $21.919(0.098)$ & $-0.537(0.294)$ & $0.291(0.087)$ & $40.584(0.286)$ \\
\hline $\mathrm{p} 425$ & $0.456(0.004)$ & $24.017(0.123)$ & $4.292(1.890)$ & $0.543(0.134)$ & $42.562(0.435)$ \\
\hline $\mathrm{p} 455$ & $0.285(0.006)$ & $21.675(0.054)$ & $-1.126(0.267)$ & $-0.010(0.051)$ & $41.026(0.211)$ \\
\hline p524 & $0.511(0.003)$ & $22.932(0.063)$ & $0.879(0.776)$ & $0.051(0.054)$ & $42.339(0.232)$ \\
\hline p528 & $0.780(0.009)$ & $24.049(0.071)$ & $-0.551(1.357)$ & $-0.074(0.131)$ & $43.617(0.394)$ \\
\hline sn90O & $0.031(0.001)$ & $16.187(0.025)$ & $0.338(0.191)$ & $-0.005(0.022)$ & $35.677(0.194)$ \\
\hline sn90af & $0.050(0.001)$ & $17.751(0.013)$ & $-2.633(0.245)$ & $0.066(0.018)$ & $36.755(0.176)$ \\
\hline $\operatorname{sn} 91 \mathrm{U}$ & $0.033(0.001)$ & $16.417(0.055)$ & $0.181(0.205)$ & $0.110(0.030)$ & $35.607(0.203)$ \\
\hline sn91ag & $0.014(0.001)$ & $14.390(0.021)$ & $0.743(0.160)$ & $0.020(0.018)$ & $33.860(0.260)$ \\
\hline sn92J & $0.046(0.001)$ & $18.660(0.036)$ & $-0.245(0.326)$ & $0.665(0.032)$ & $36.429(0.194)$ \\
\hline sn92P & $0.026(0.001)$ & $16.043(0.018)$ & $0.168(0.351)$ & $-0.009(0.021)$ & $35.524(0.202)$ \\
\hline sn92ae & $0.075(0.001)$ & $18.417(0.023)$ & $-0.811(0.193)$ & $0.022(0.026)$ & $37.719(0.178)$ \\
\hline sn92ag & $0.026(0.001)$ & $16.280(0.021)$ & $-0.530(0.175)$ & $0.221(0.021)$ & $35.121(0.201)$ \\
\hline sn92al & $0.014(0.001)$ & $14.546(0.012)$ & $-0.295(0.073)$ & $-0.027(0.013)$ & $34.023(0.254)$ \\
\hline sn92aq & $0.101(0.001)$ & $19.267(0.048)$ & $-1.364(0.404)$ & $-0.021(0.034)$ & $38.619(0.192)$ \\
\hline sn92bc & $0.020(0.001)$ & $15.068(0.009)$ & $0.310(0.073)$ & $-0.060(0.010)$ & $34.691(0.214)$ \\
\hline sn92bh & $0.045(0.001)$ & $17.586(0.022)$ & $-0.068(0.217)$ & $0.101(0.021)$ & $36.771(0.181)$ \\
\hline sn92bk & $0.058(0.001)$ & $18.036(0.123)$ & $-2.105(0.322)$ & $-0.045(0.076)$ & $37.370(0.281)$ \\
\hline
\end{tabular}


Table 2-Continued

\begin{tabular}{|c|c|c|c|c|c|}
\hline SN Ia & $B$ & $m_{B}$ & $x_{1}$ & $c$ & $\mu^{1}$ \\
\hline sn92bl & $0.043(0.001)$ & $17.299(0.055)$ & $-2.013(0.221)$ & $0.014(0.036)$ & $36.497(0.202)$ \\
\hline sn92bp & $0.079(0.001)$ & $18.276(0.014)$ & $-1.258(0.190)$ & $-0.026(0.017)$ & $37.652(0.169)$ \\
\hline sn92br & $0.088(0.001)$ & $19.618(0.037)$ & $-2.572(0.293)$ & $0.209(0.051)$ & $38.275(0.211)$ \\
\hline sn92bs & $0.063(0.001)$ & $18.267(0.029)$ & $-0.389(0.188)$ & $0.024(0.019)$ & $37.610(0.174)$ \\
\hline sn93B & $0.071(0.001)$ & $18.447(0.018)$ & $-0.667(0.196)$ & $0.104(0.021)$ & $37.563(0.173)$ \\
\hline sn93O & $0.052(0.001)$ & $17.612(0.013)$ & $-0.948(0.100)$ & $-0.015(0.014)$ & $36.993(0.172)$ \\
\hline sn93ac & $0.049(0.001)$ & $17.717(0.037)$ & $-1.159(0.310)$ & $0.060(0.030)$ & $36.891(0.190)$ \\
\hline sn93ag & $0.050(0.001)$ & $17.789(0.018)$ & $-1.068(0.199)$ & $0.114(0.022)$ & $36.839(0.179)$ \\
\hline sn94M & $0.024(0.001)$ & $16.274(0.021)$ & $-1.746(0.174)$ & $0.104(0.021)$ & $35.276(0.205)$ \\
\hline sn94S & $0.016(0.001)$ & $14.762(0.021)$ & $0.226(0.260)$ & $-0.002(0.019)$ & $34.232(0.242)$ \\
\hline sn94T & $0.036(0.001)$ & $16.914(0.027)$ & $-1.489(0.168)$ & $0.030(0.024)$ & $36.126(0.189)$ \\
\hline sn95ac & $0.049(0.001)$ & $17.039(0.011)$ & $0.591(0.119)$ & $0.011(0.010)$ & $36.516(0.171)$ \\
\hline sn95ak & $0.022(0.001)$ & $15.935(0.046)$ & $-1.376(0.2$ & $0.079(0.030)$ & $35.038(0.223)$ \\
\hline sn95bd & $0.014(0.001)$ & $15.233(0.015)$ & $0.598(0.1$ & $0.328(0.015)$ & 33.926( \\
\hline sn96C & $0.028(0.001)$ & 16.5 & 0.421 & $0.116(0$ & \\
\hline sn96ab & $0.124(0.001)$ & $19.540(0.024)$ & $-0.907(0.252)$ & $-0.062(0.024)$ & $39.043(0.174)$ \\
\hline sn96bl & $0.035(0.001)$ & $16.673(0$. & $-0.181(0.1$ & $0.055(0.015)$ & $35.961(0.183)$ \\
\hline sn96bo & $0.016(0.001)$ & $15.821(0.0$ & $-0.968(0.160)$ & $0.397(0.014)$ & 34.179 \\
\hline sn96bv & $0.017(0.001)$ & $15.277(0.035)$ & $0.936(0.418)$ & $0.190(0.022)$ & $34.347(0.243)$ \\
\hline sn97E & $0.013(0.001)$ & $15.093(0.015)$ & $-1.830(0.248)$ & $0.089(0.015)$ & $34.124(0.265)$ \\
\hline $\operatorname{sn} 97 \mathrm{Y}$ & $0.017(0.001)$ & $15.303(0$ & $-1.264(0.277)$ & $0.041(0$ & $34.512(0.240)$ \\
\hline sn97dg & $0.030(0.001)$ & $16.805(0.016)$ & $-0.869(0.260)$ & $0.002(0.015)$ & $36.151(0.191)$ \\
\hline sn97do & $0.011(\mathrm{C}$ & 14.3 & -0.212( & 0.13 & 33.408 \\
\hline sn98V & $0.017(c$ & 15.0 & -0.3 & 0.03 & 34.404 \\
\hline sn98ab & $0.028(0.001)$ & $16.090(0.017)$ & $0.130(0.116)$ & $0.108(0$ & $35.278(0.192)$ \\
\hline $\operatorname{sn} 98 d x$ & $0.054(0.001)$ & $17.510(0.033)$ & $-1.713(0.387)$ & $-0.073(0.021)$ & $36.956(0.182)$ \\
\hline sn98ef & $0.017(0.001)$ & $14.806(0.025)$ & $-1.389(0.257)$ & $-0.005(0.018)$ & $34.117(0.237)$ \\
\hline sn98eg & $0.024(0.001)$ & 16.077( & $-0.822(0.3$ & $0.047(0.018)$ & $35.317(0.207)$ \\
\hline sn99aa & $0.015(0.001)$ & $14.694(0.009)$ & $0.981(0.073)$ & $-0.015(0.009)$ & $34.277(0.242)$ \\
\hline sn99aw & $0.039(0.001)$ & $16.710(0.017)$ & $2.064(0.186)$ & $-0.011(0.017)$ & $36.395(0.181)$ \\
\hline sn99cc & $0.032(0.001)$ & $16.754(0.009)$ & $-1.756(0.161)$ & $0.063(0.011)$ & $35.857(0.185)$ \\
\hline sn99cp & 0.010 & 13.928 & $-0.043(0$. & $-0.019(0.0$ & $33.412(0.310)$ \\
\hline sn99dk & $0.014(0.001)$ & $14.882(0.015)$ & $0.801(0.118)$ & $0.160(0.013)$ & $34.011(0.257)$ \\
\hline sn99dq & $0.013(0.001)$ & $14.348(0.007)$ & $0.287(0.034)$ & $0.118(0.008)$ & $33.529(0.259)$ \\
\hline sn99ee & $0.011(0.001)$ & 14.8 & & $0.328(0.005)$ & $33.526(0.302)$ \\
\hline sn99ef & $0.038(0.001)$ & $17.029(0.055)$ & $0.308(0.322)$ & $-0.003(0.022)$ & $36.510(0.194)$ \\
\hline sn99ej & $0.013(0.001)$ & $15.345(0.088)$ & $-2.093(0.401)$ & $0.042(0.045)$ & $34.465(0.305)$ \\
\hline sn99ek & $0.018(0.001)$ & $15.588(0.008)$ & $-0.999(0.116)$ & $0.170(0.009)$ & $34.505(0.225)$ \\
\hline sn99gd & $0.019(0.001)$ & $16.915(0.030)$ & $-1.114(0.226)$ & $0.460(0.021)$ & $35.102(0.224)$ \\
\hline sn99gp & $0.026(0.001)$ & $15.984(0.009)$ & $1.443(0.094)$ & $0.057(0.010)$ & $35.437(0.194)$ \\
\hline sn00bh & $0.024(0.001)$ & $15.928(0.040)$ & $-0.536(0.124)$ & $0.075(0.021)$ & $35.130(0.207)$ \\
\hline sn00ca & $0.025(0.001)$ & $15.525(0.013)$ & $0.261(0.127)$ & $-0.074(0.011)$ & $35.178(0.198)$ \\
\hline sn00ce & $0.017(0.001)$ & $17.008(0.022)$ & $0.613(0.179)$ & $0.579(0.017)$ & $35.081(0.237)$ \\
\hline sn00cf & $0.036(0.001)$ & $17.020(0.024)$ & $-0.836(0.129)$ & $0.017(0.015)$ & $36.332(0.182)$ \\
\hline sn00cn & $0.023(0.001)$ & $16.573(0.018)$ & $-2.380(0.243)$ & $0.199(0.016)$ & $35.277(0.206)$ \\
\hline sn00dk & $0.016(0.001)$ & $15.347(0.016)$ & $-2.496(0.267)$ & $0.062(0.016)$ & $34.376(0.238)$ \\
\hline
\end{tabular}


Table 2-Continued

\begin{tabular}{|c|c|c|c|c|c|}
\hline SN Ia & 3 & $m_{B}$ & $x_{1}$ & $c$ & $\mu^{1}$ \\
\hline sn00fa & $0.022(0.001)$ & $15.864(0.021)$ & $0.361(0.130)$ & $0.099(0.015)$ & $35.098(0.209)$ \\
\hline sn01ah & $0.058(0.001)$ & $17.608(0.026)$ & $1.221(0.303)$ & $0.025(0.022)$ & 37.116 \\
\hline sn01az & $0.041(0.001)$ & $16.891(0.029)$ & $0.472(0.347)$ & $-0.044(0.016)$ & $36.492(0.183)$ \\
\hline sn01ba & $0.030(0.001)$ & $16.163(0.009)$ & $-0.038(0.126)$ & $-0.096(0.011)$ & $35.840(0.186)$ \\
\hline sn01bf & $0.015(0.001)$ & $14.682(0.016)$ & $0.718(0.146)$ & $0.011(0.012)$ & $34.172(0.244)$ \\
\hline sn01bt & $0.014(0.001)$ & $15.289(0.011)$ & $-0.782(0.063)$ & $0.259(0.012)$ & $34.009(0.251)$ \\
\hline sn01cn & $0.015(0.001)$ & $15.237(0.015)$ & $-0.445(0.060)$ & $0.203(0.011)$ & $34.129(0.242)$ \\
\hline sn01cp & $0.022(0.001)$ & $15.110(0.176)$ & $-1.851(1.162)$ & $-0.341(0.106)$ & $35.204(0.394)$ \\
\hline sn01cz & $0.016(0.001)$ & $15.029(0.012)$ & $-0.077(0.118)$ & $0.125(0.012)$ & $34.154(0.235)$ \\
\hline sn01da & $0.016(0.001)$ & $15.366(0.034)$ & $-4.263(0.815)$ & $0.033(0.036)$ & $34.284(0.267)$ \\
\hline sn01eh & $0.036(0.001)$ & $16.572(0.014)$ & $1.159(0.112)$ & $0.016(0.013)$ & $36.095(0.180)$ \\
\hline sn01en & $0.015(0.001)$ & $15.062(0.047)$ & $-1.130(0.1$ & $0.085(0.025)$ & $34.176(0.252)$ \\
\hline sn01ep & $0.013(0.001)$ & $14.869(0.012)$ & $-1.200(0$ & $0.132(0$ & 33.859 \\
\hline sn01fe & $0.014(0.001)$ & $14.636(0.0$ & 0.360 & 0015 & 54) \\
\hline sn01fh & 0.01 & 1) & -2.5 & -0.0 & \\
\hline sn01ie & $0.031(0.001)$ & $16.576(0.055)$ & $-1.119(0.2$ & & \\
\hline $\operatorname{sn} 01 \mathrm{~N}$ & $0.022(0.0$ & $16.552(0.029)$ & $-0.602(0.2$ & $0.369(0.021)$ & $35.017(0.213)$ \\
\hline sn02bf & $0.025(0.001)$ & $16.305(0.040)$ & $-0.389(0$ & $0.228(0.0$ & 35.143 \\
\hline & 0.03 & 16.9 & -1.718 & $-0.073(C$ & \\
\hline sn02ck & $0.030(0.001)$ & $16.289(0.015)$ & & $-0.018(0.015)$ & \\
\hline $\mathrm{sn} 02 \mathrm{cr}$ & 0.010 & 14.191 & -0.652( & $0.031(c$ & $33.489(0.311)$ \\
\hline sn02de & $0.028(0.001)$ & $16.624(0.013)$ & $-0.042(0.3$ & $0.164(0.0$ & 35.6 \\
\hline sn02dj & & & & & \\
\hline sno & 0.01 & & & & \\
\hline sn02dp & $0.011(0.001)$ & $14.548(0.014)$ & $-0.654(($ & $0.145(0$. & $33.562(0.307)$ \\
\hline sn02es & $0.018(0.001)$ & $16.573(0.040)$ & $-1.844(0.264)$ & $0.408(0.028)$ & $34.815(0.240)$ \\
\hline sn02G & $0.035(0.001)$ & $17.511(0.076)$ & $-2.017(0.707)$ & $0.250(0.048)$ & $36.125(0.239)$ \\
\hline sn02ha & $0.001)$ & 14.6 & -1.731 & $-0.029(0.018)$ & $34.006(0.266)$ \\
\hline sn02hd & $0.036(0.001)$ & $16.709(0.016)$ & $-0.776(0.439)$ & $0.092(0.015)$ & $35.843(0.187)$ \\
\hline sn02he & $0.025(0.001)$ & $16.231(0.039)$ & $-2.118(0.268)$ & $0.012(0.026)$ & $35.424(0.210)$ \\
\hline sn02hu & $0.038(0.001)$ & $16.582(0.011)$ & $0.096(0.134)$ & $-0.052(0.010)$ & $36.163(0.177)$ \\
\hline sn02hw & $0.016(0.0$ & 16.681(C & -2.245( & $0.531 / 0$ & 34.574 \\
\hline sn02jy & $0.020(0.001)$ & $15.690(0.013)$ & & $0.011(0$ & $35.190(0.214)$ \\
\hline sn02kf & $0.019(0.001)$ & $15.651(0.027)$ & $-1.370(0.164)$ & $0.023(0.018)$ & $34.893(0.220)$ \\
\hline $\mathrm{sn} 03 \mathrm{U}$ & 0.02 & 16.4 & & $0.030(0.0$ & 35.5 \\
\hline sn03W & $0.021(0.001)$ & 15.8 & -0.2 & 0.190 & 34.79 \\
\hline sn03ch & $0.030(0.001)$ & $16.654(0.017)$ & $-1.630(0.213)$ & $0.019(0.017)$ & $35.879(0.192)$ \\
\hline sn03cq & $0.034(0.001)$ & $17.208(0.047)$ & $-0.985(0.239)$ & $0.227(0.041)$ & $35.985(0.213)$ \\
\hline sn03fa & $0.039(0.001)$ & $16.642(0.016)$ & $1.128(0.134)$ & $-0.005(0.013)$ & $36.215(0.178)$ \\
\hline sn03hu & $0.075(0.001)$ & $18.403(0.103)$ & $0.872(0.420)$ & $0.166(0.036)$ & $37.525(0.217)$ \\
\hline sn03ic & $0.054(0.001)$ & $17.630(0.026)$ & $-2.497(0.319)$ & $0.018(0.027)$ & $36.769(0.184)$ \\
\hline sn03it & $0.024(0.001)$ & $16.334(0.025)$ & $-1.738(0.341)$ & $0.086(0.027)$ & $35.383(0.212)$ \\
\hline sn03iv & $0.034(0.001)$ & $16.956(0.024)$ & $-2.422(0.468)$ & $-0.024(0.025)$ & $36.204(0.198)$ \\
\hline sn04as & & & $-0.137(0.166)$ & $0.134(0.01$ & $36.044(0.186)$ \\
\hline sn04bg & $0.022(0.001)$ & $15.528(0.050)$ & $0.403(0.172)$ & $-0.001(0.026)$ & $35.015(0.220)$ \\
\hline sn04L & $0.033(0.001)$ & $17.377(0.034)$ & $-1.147(0.306)$ & $0.285(0.025)$ & $35.993(0.196)$ \\
\hline
\end{tabular}


Table 2-Continued

\begin{tabular}{|c|c|c|c|c|c|}
\hline SN Ia & $z_{C M B}$ & $m_{B}$ & $x_{1}$ & $c$ & $\mu^{1}$ \\
\hline $\mathrm{sn} 05 \mathrm{dv}$ & $0.011(0.001)$ & $16.280(0.030)$ & $0.696(1.226)$ & $0.789(0.020)$ & $33.842(0.333)$ \\
\hline sn05el & $0.015(0.001)$ & $14.804(0.013)$ & $-1.747(0.077)$ & $-0.065(0.013)$ & $34.225(0.248)$ \\
\hline sn05eq & $0.028(0.001)$ & $16.223(0.012)$ & $0.820(0.093)$ & $0.034(0.010)$ & $35.668(0.189)$ \\
\hline sn05eu & $0.034(0.001)$ & $16.361(0.024)$ & $0.686(0.122)$ & $-0.064(0.021)$ & $36.034(0.188)$ \\
\hline sn05hc & $0.045(0.001)$ & $17.311(0.010)$ & $-0.022(0.140)$ & $0.008(0.009)$ & $36.731(0.173)$ \\
\hline sn05hf & $0.042(0.001)$ & $17.232(0.042)$ & $-2.382(0.264)$ & $-0.051(0$. & $36.552(0.196)$ \\
\hline sn05hj & $0.057(0.001)$ & $17.661(0.027)$ & $1.707(0.337)$ & $-0.019(0.018)$ & $37.328(0.177)$ \\
\hline sn05iq & $0.033(0.001)$ & $16.787(0.015)$ & $-1.556(0.187)$ & $-0.041(0.014)$ & $36.169(0.185)$ \\
\hline sn05ir & $0.075(0.001)$ & $18.329(0.029)$ & $2.979(0.540)$ & $-0.069(0.030)$ & $38.252(0.190)$ \\
\hline $\mathrm{sn} 05 \mathrm{kc}$ & $0.014(0.001)$ & $15.616(0.012)$ & $-0.747(0.100)$ & $0.320(0$. & $34.187(0.257)$ \\
\hline sn05ki & $0.021(0.001)$ & $15.546(0.021)$ & $-2.077(0.148)$ & $-0.058(0.018)$ & $34.914(0.214)$ \\
\hline sn05ls & $0.021(0.001)$ & $16.151(0.018)$ & $0.574(0.191)$ & $0.372(0.013)$ & $34.732(0.213)$ \\
\hline sn05lz & $0.040(0.001)$ & $17.590(0.015)$ & $-1.461(0.188)$ & $0.127(0.015)$ & $36.567(0.179)$ \\
\hline $\mathrm{sn} 05 \mathrm{mc}$ & $0.026(0.001)$ & $17.236(0.018)$ & $-3.041(0.242)$ & $0.309(0.017)$ & $35.597(0.199)$ \\
\hline $\mathrm{sn} 05 \mathrm{~ms}$ & $0.026(0.001)$ & $16.107(0.011)$ & $-0.052(0.091)$ & $0.012(0$. & $35.514(0.194)$ \\
\hline sn05na & $0.027(0.001)$ & 16.099 & -0.730( & 0.012( & 35.436 \\
\hline sn06ac & $0.024(0.001)$ & $16.164(0.012)$ & $-0.968(0.0$ & & $35.193(0.201)$ \\
\hline sn06ak & $0.039(0.001)$ & $17.173(0.074)$ & $-1.652(0$ & $0.047(0.045)$ & 36.328 \\
\hline sn06al & $0.069(0.001)$ & $18.395(0.027)$ & $-2.277(0.327)$ & $-0.012(0.025)$ & $37.630(0.180)$ \\
\hline sn06an & 0.065 & $18.034(0.0$ & 0.186 & $0.026(0.016)$ & 37.430 \\
\hline sn06ar & $0.023(0.001)$ & $16.450(0.013)$ & $-0.828(0.294)$ & $0.177(0.014)$ & 35.367 \\
\hline sn06ax & $0.018(0.001)$ & $15.006(0.008)$ & $-0.052(0.057)$ & $-0.039(0.008)$ & 34.54 \\
\hline sn06az & $0.032(0.001)$ & $16.436(0.012)$ & $-1.758(0.090)$ & $-0.052(0.011)$ & $35.824(0.184)$ \\
\hline sn06bq & $0.021(0.001)$ & $16.218(0.0$ & $-1.900(0.115)$ & $0.162(0.0$ & $35.062(0.208)$ \\
\hline sn06bt & 0.03 & 16. & 0.069 & 0.1 & 35. \\
\hline sn06bu & 0.084 & 18.13 & 2.34 & -0.12 & 38.1 \\
\hline sn06bw & $0.031(0.001)$ & $17.476(0.052)$ & $-2.173(0.272)$ & $0.369(0.029)$ & $35.778(0.206)$ \\
\hline $\mathrm{sn} 06 \mathrm{cc}$ & $0.033(0.001)$ & $17.766(0.0$ & $0.092(0$. & 0.40 & 36.22 \\
\hline sn06cf & 0.04 & $17.091(0$ & $-1.166(0.415)$ & $-0.020(0.028)$ & $36.462(0.195)$ \\
\hline sn06cg & $0.029(0.001)$ & $16.448(0.092)$ & $-2.379(0.576)$ & $0.191(0.058)$ & $35.170(0.260)$ \\
\hline sn06cj & $0.068(0.001)$ & $18.087(0.027)$ & $1.475(0.590)$ & $-0.075(0.017)$ & $37.868(0.182)$ \\
\hline sn06cp & $0.023(0.001)$ & $15.973(0.015)$ & $-0.072(0.100)$ & $0.153(0.013)$ & $35.028(0.202)$ \\
\hline sn06cq & $0.049(0.001)$ & $17.536(0$ & $-0.009(0$ & $0.035(c$ & $36.890(0.179)$ \\
\hline sn06ef & $0.017(0.001)$ & $15.573(0$ & $-1.306(0$ & $0.038(C$ & $34.785(0.243)$ \\
\hline sn06ej & $0.019(0.001)$ & $15.747(0.034)$ & $-1.552(0.162)$ & $0.048(0.026)$ & $34.910(0.227)$ \\
\hline sn06en & $0.031(0.001)$ & $16.761(0.036)$ & $-0.824(0.143)$ & $0.082(0.020)$ & $35.914(0.193)$ \\
\hline sn06et & 0.021 & 15.9 & $0.626(C$ & $0.201(c$ & $34.951(0.214)$ \\
\hline sn06gj & $0.028(0.001)$ & $17.651(0.030)$ & $-2.171(0.275)$ & $0.408(0.022)$ & $35.858(0.200)$ \\
\hline sn06gr & $0.034(0.001)$ & $16.909(0.010)$ & $0.405(0.093)$ & $0.113(0.009)$ & $36.115(0.181)$ \\
\hline sn06kf & $0.021(0.001)$ & $15.806(0.026)$ & $-2.860(0.208)$ & $-0.031(0.025)$ & $35.028(0.219)$ \\
\hline sn06le & $0.017(0.001)$ & $14.802(0.012)$ & $1.452(0.091)$ & $-0.054(0.011)$ & $34.528(0.228)$ \\
\hline sn06lf & $0.013(0.001)$ & $13.629(0.021)$ & $-1.938(0.119)$ & $-0.208(0.019)$ & $33.385(0.270)$ \\
\hline $\mathrm{sn} 06 \mathrm{mo}$ & $0.036(0.001)$ & $17.404(0.041)$ & $-2.658(0.250)$ & $0.101(0.033)$ & $36.320(0.200)$ \\
\hline $\mathrm{sn} 06 \mathrm{~N}$ & $0.014(0.001)$ & $15.130(0.016)$ & $-2.439(0.122)$ & $0.010(0.014)$ & $34.294(0.253)$ \\
\hline sn06nz & $0.037(0.001)$ & $18.096(0.036)$ & $-3.484(0.971)$ & $0.305(0.032)$ & $36.421(0.220)$ \\
\hline sn06oa & $0.059(0.001)$ & $17.830(0.017)$ & $0.756(0.283)$ & $0.038(0.012)$ & $37.257(0.171)$ \\
\hline
\end{tabular}


Table 2-Continued

\begin{tabular}{|c|c|c|c|c|c|}
\hline SN Ia & $z_{C M B}$ & $m_{B}$ & $x_{1}$ & $c$ & $\mu^{1}$ \\
\hline sn06ob & $0.058(0.001)$ & $18.202(0.016)$ & $-2.841(0.242)$ & $0.043(0.017)$ & $37.243(0.174)$ \\
\hline sn06on & $0.069(0.001)$ & $18.366(0.039)$ & $0.427(0.536)$ & $0.104(0.036)$ & $37.595(0.198)$ \\
\hline sn06os & $0.032(0.001)$ & $17.613(0.022)$ & $-0.917(0.235)$ & $0.480(0.019)$ & $35.773(0.190)$ \\
\hline sn06qo & $0.030(0.001)$ & $16.792(0.014)$ & $0.199(0.089)$ & $0.220(0.012)$ & $35.712(0.187)$ \\
\hline sn06S & $0.033(0.001)$ & $16.804(0.010)$ & $0.547(0.103)$ & $0.098(0.010)$ & $36.062(0.182)$ \\
\hline sn06sr & $0.023(0.001)$ & $16.120(0.015)$ & $-1.628(0.209)$ & $0.061(0.013)$ & $35.241(0.204)$ \\
\hline sn06td & $0.015(0.001)$ & $15.714(0.018)$ & $-1.719(0.201)$ & $0.170(0.018)$ & $34.556(0.249)$ \\
\hline sn06te & $0.032(0.001)$ & $16.576(0.012)$ & $-0.701(0.093)$ & $-0.017(0.012)$ & $35.986(0.184)$ \\
\hline sn07ae & $0.064(0.001)$ & $17.736(0.042)$ & $1.050(0.351)$ & $0.020(0.022)$ & $37.238(0.182)$ \\
\hline sn07ai & $0.032(0.001)$ & $16.871(0.023)$ & $1.205(0.395)$ & $0.213(0.023)$ & $35.912(0.195)$ \\
\hline sn07ap & $0.016(0.001)$ & $15.586(0.037)$ & $-1.981(0.140)$ & $0.056(0.030)$ & $34.683(0.248)$ \\
\hline sn07au & $0.020(0.001)$ & $16.616(0.035)$ & $-3.419(0.272)$ & $0.329(0.030)$ & $34.888(0.227)$ \\
\hline sn07bc & $0.022(0.001)$ & $15.854(0.019)$ & $-1.669(0.160)$ & $0.032(0.018)$ & $35.043(0.210)$ \\
\hline sn07bd & $0.032(0.001)$ & $16.539(0.015)$ & $-1.818(0.153)$ & $-0.002(0.016)$ & $35.798(0.187)$ \\
\hline $\mathrm{sn} 07 \mathrm{bz}$ & $0.023(0.001)$ & $16.611(0.029)$ & $1.199(0.212)$ & $0.162(0.022)$ & $35.777(0.212)$ \\
\hline sn07ca & $0.015(0.001)$ & $15.910(0.012)$ & $0.211(0.112)$ & $0.298(0.012)$ & $34.636(0.244)$ \\
\hline sn07cg & $0.034(0.001)$ & $18.155(0.062)$ & $-0.868(0.453)$ & $0.671(0.029)$ & $35.845(0.207)$ \\
\hline $\mathrm{sn} 07 \mathrm{ci}$ & $0.019(0.001)$ & $15.910(0.032)$ & $-3.182(0.247)$ & $0.134(0.028)$ & $34.691(0.230)$ \\
\hline $\mathrm{sn} 07 \mathrm{co}$ & $0.027(0.001)$ & $16.456(0.007)$ & $-0.468(0.088)$ & $0.150(0.007)$ & $35.477(0.192)$ \\
\hline sn07cq & $0.025(0.001)$ & $15.799(0.011)$ & $-0.692(0.163)$ & $0.034(0.010)$ & $35.086(0.198)$ \\
\hline $\mathrm{sn} 07 \mathrm{~F}$ & $0.024(0.001)$ & $15.853(0.009)$ & $0.339(0.067)$ & $-0.008(0.009)$ & $35.351(0.198)$ \\
\hline $\mathrm{sn} 07 \mathrm{H}$ & $0.044(0.001)$ & $18.240(0.126)$ & $-0.248(0.260)$ & $0.370(0.063)$ & $36.740(0.265)$ \\
\hline sn07O & $0.036(0.001)$ & $16.815(0.009)$ & $-1.093(0.111)$ & $0.029(0.013)$ & $36.071(0.181)$ \\
\hline sn07qe & $0.023(0.001)$ & $16.019(0.009)$ & $0.482(0.070)$ & $0.098(0.009)$ & $35.268(0.197)$ \\
\hline sn07R & $0.031(0.001)$ & $16.629(0.043)$ & $-1.825(0.166)$ & $-0.061(0.025)$ & $36.032(0.201)$ \\
\hline sn07S & $0.015(0.001)$ & $15.810(0.010)$ & $0.827(0.097)$ & $0.473(0.010)$ & $34.168(0.258)$ \\
\hline sn08L & $0.019(0.001)$ & $15.104(0.045)$ & $-2.040(0.250)$ & $-0.084(0.037)$ & $34.542(0.242)$ \\
\hline sn08af & $0.034(0.001)$ & $17.314(0.017)$ & $-1.979(0.205)$ & $0.261(0.020)$ & $35.903(0.187)$ \\
\hline sn08bf & $0.026(0.001)$ & $15.696(0.010)$ & $0.170(0.097)$ & $0.032(0.010)$ & $35.075(0.195)$ \\
\hline
\end{tabular}

${ }^{1}$ Distance moduli were calculated with $\mathrm{H}_{\mathrm{o}}=65 \mathrm{kms}^{-1}, M_{B}=-19.44, \alpha=0.104_{-0.018}^{+0.018}$ and $\beta=2.48_{-0.12}^{+0.10}$. Uncertainty of $\mu$ includes $0.158 \mathrm{mag}$ "intrinsic", $\sigma_{v-p e c}\left(400 \mathrm{~km} \mathrm{~s}^{-1}\right)$, and $\sigma_{z}$. 
Table 3. MLCS31 Light Curve Fit Parameters.

\begin{tabular}{|c|c|c|c|c|}
\hline SN Ia & $z_{C M B}$ & $\Delta$ & $A_{V}$ & $\mu^{1}$ \\
\hline 03D1au & $0.504(0.001)$ & $-0.296(0.065)$ & $0.147(0.127)$ & $42.540(0.179)$ \\
\hline 03D1aw & $0.582(0.001)$ & $-0.262(0.1$ & $.144(0.113)$ & $43.098(0.207)$ \\
\hline 03D1ax & & $.178(0$ & $117(0$ & $42.322(0.194)$ \\
\hline 03D1bp & $0.346(0.001)$ & $157(0.121)$ & $0.268(0.224)$ & $41.516(0.255)$ \\
\hline 03D1co & $0.679(0.001)$ & $-0.244(0.1$ & $0.172(0.162)$ & $43.589(0.261)$ \\
\hline 03D1ew & $0.868(0.001)$ & $-0.306(0.099)$ & $0.095(0.071)$ & $43.951(0.159)$ \\
\hline 03D1fc & & & & \\
\hline 03D1fl & 0.688( & $-0.225(0.1$ & $.136(0$ & 43.123 \\
\hline 03D1fq & 0.800 & $0.077(C$ & 0.115( & 43.922 \\
\hline 03D1gt & $0.548(0.001)$ & $0.379(0.294)$ & $0.702(0.392)$ & $42.400(0.421)$ \\
\hline 03D3af & $0.532(0.001)$ & $-0.017(0.1$ & $0.209(0$ & $42.827(0.291)$ \\
\hline 03D3aw & 0.449 & $-0.005(C$ & $0.076(0$ & 42.072 \\
\hline 03D3ay & 0.37 & -0.096( & 0.065 & 41.799 \\
\hline 03D3ba & $0.291(0.001)$ & $-0.097(0.0$ & $0.845(0.279)$ & $40.546(0.307)$ \\
\hline 03D3cc & 0.463 & -0.176( & 0.093 & 42.264 \\
\hline 03D3cd & 0.461 & -0.214 & 0.078 & 42.12 \\
\hline 03D4ag & & -0. & & \\
\hline 03D4at & & & & \\
\hline $03 \mathrm{D} 4 \mathrm{cx}$ & 0.949 & -0.272( & & 44.232 \\
\hline 03D4cy & 0.9 & & 0.061 & 4 \\
\hline $03 \mathrm{D} 4 \mathrm{cz}$ & 0.6 & 92( & 0.177 & 43.098 \\
\hline $03 \mathrm{D}$ & 0.6 & -0.244( & & 42.94 \\
\hline 03D4di & 0.90 & -0.281( & 0.074 & \\
\hline 03D4dy & $0.604(C$ & $-0.300(C$ & 0.263 & $42.740(0.327)$ \\
\hline 03D4fd & $0.791(0.0$ & $-0.229(0.1$ & $0.121(0.1$ & $43.752(0.204)$ \\
\hline 03D4gf & 0.5 & -0.041 & 0.073 & 42.850 \\
\hline $03 \mathrm{D}$ & 0.5 & -0.139 & 0.126 & \\
\hline 03D4gl & $0.571(0.001)$ & $0.149(0$ & $0.143(0.144)$ & $42.463(0.365)$ \\
\hline 04D1ag & & $-0.112(0.2$ & $0.083(0.0$ & 42.62 \\
\hline $04 \mathrm{I}$ & & -0.281( & 0.136 & 43.44 \\
\hline $04 \mathrm{D}$ & 0.52 & 0.490( & 0.228 & 42.470 \\
\hline 04D2cf & $0.369(0.001)$ & $0.067(0.114)$ & $0.118(0.101)$ & $41.805(0.162)$ \\
\hline 04D2fp & $0.415(0.001)$ & $-0.113(0.088)$ & $0.124(0.101)$ & $42.053(0.159)$ \\
\hline $04 \mathrm{D} 2 \mathrm{fs}$ & & -0.089( & 0.327 & 41.622 \\
\hline 04D2gb & 0.43 & $0.564(0.107)$ & 0.110 & $41.791(0.168)$ \\
\hline 04D2gp & $0.707(0.001)$ & $0.181(0.234)$ & $0.137(0.143)$ & $43.430(0.322)$ \\
\hline 04D2iu & $0.691(0.001)$ & $0.302(0.243)$ & $0.180(0.171)$ & $43.401(0.385)$ \\
\hline 04D2ja & & & & $43.579(0.233)$ \\
\hline 04D3co & $0.620(0.001)$ & $0.016(0.150)$ & $0.153(0.128)$ & $43.208(0.252)$ \\
\hline 04D3cp & $0.830(0.001)$ & $-0.285(0.074)$ & $0.080(0.070)$ & $43.606(0.153)$ \\
\hline 04D3cy & $0.643(0.001)$ & $-0.207(0.108)$ & $0.147(0.119)$ & $43.331(0.211)$ \\
\hline 04D3dd & $1.010(0.001)$ & $-0.313(0.118)$ & $0.042(0.032)$ & $44.695(0.159)$ \\
\hline 04D3df & 0.47 & $0.737(0.1$ & 0.25 & $42.003(0.200)$ \\
\hline 04D3do & $0.610(0.001)$ & $0.228(0.153)$ & $0.144(0.155)$ & $42.816(0.284)$ \\
\hline 04D3ez & $0.263(0.001)$ & $0.026(0.072)$ & $0.399(0.158)$ & $40.739(0.196)$ \\
\hline 04D3fk & $0.358(0.001)$ & $-0.066(0.077)$ & $0.614(0.163)$ & $41.393(0.200)$ \\
\hline
\end{tabular}


Table 3-Continued

\begin{tabular}{|c|c|c|c|c|}
\hline SN Ia & $z_{C M B}$ & $\Delta$ & $A_{V}$ & $\mu^{1}$ \\
\hline 04D3fq & $0.730(0.001)$ & $-0.038(0.185)$ & $0.127(0.125)$ & $43.571(0.256)$ \\
\hline 04D3gt & $451(0.001)$ & $0.012(0.086)$ & $1.147(0.184)$ & $41.336(0.225)$ \\
\hline 04D3gx & $0.910(0.001)$ & $-0.162(0.136)$ & $0.064(0.054)$ & $44.205(0.165)$ \\
\hline 04D3hn & $0.552(0.001)$ & $0.089(0.127)$ & $0.635(0.304)$ & $42.200(0.384)$ \\
\hline 04D3is & $0.710(0.001)$ & $-0.323(0.115)$ & $0.264(0.231)$ & $43.721(0.341)$ \\
\hline 04D3ki & $0.930(0.001)$ & $-0.154(0.1$ & $0.071(0$ & \\
\hline 04D3kr & $0.337(0.001)$ & $-0.238(0.066)$ & $0.157(0.121)$ & $41.443(0.160)$ \\
\hline 04D3ks & $0.752(0.001)$ & $-0.223(0.116)$ & $0.151(0.127)$ & $43.362(0.222)$ \\
\hline 04D3lu & $0.822(0.001)$ & $-0.026(0.135)$ & $0.111(0.089)$ & $43.766(0.207)$ \\
\hline 04D3ml & $0.950(0.001)$ & $-0.328(0.1$ & $0.054(0.046)$ & $44.154(0.146)$ \\
\hline 04D3nc & $0.817(0.001)$ & $-0.201(0.140)$ & $0.116(0.093)$ & $43.726(0.194)$ \\
\hline 04D3nh & $0.340(0.001)$ & $-0.202(0.090)$ & $0.158(0.113)$ & $41.612(0.160)$ \\
\hline 04D3nr & $0.960(0.001)$ & $-0.241(0.144)$ & $0.049(0.044)$ & $44.344(0.179)$ \\
\hline 04D3ny & $0.810(0.001)$ & $0.042(0.196)$ & $0.120(0.117)$ & $43.647(0.261)$ \\
\hline 04D3oe & $0.756(0.0$ & $-0.046(c$ & $0.094(0$. & 43.543 \\
\hline 04D4an & 0.613 & 0.290 & 0.237( & 43.063 \\
\hline 04D4bk & 0.840 & -0.281( & & 43.879 \\
\hline 04D4bq & $0.550(0.001)$ & $-0.212(0$ & $0.237(0$ & 42.728 \\
\hline 04D4dm & 0.811 & -0.169 & $0.118(0$ & $43.917(0.233)$ \\
\hline $2002 \mathrm{dc}$ & & 0.184( & 0.195 & 42.355 \\
\hline $2002 \mathrm{dd}$ & $0.950(0.0$ & $-0.254(0.125)$ & $0.230(0.2$ & 44.075 \\
\hline Eagle & $1.020(0.010)$ & $-0.129(0.1$ & $0.170(0$. & $44.418(0.222)$ \\
\hline Fergus & $1.020(0.010)$ & $0.062(0$. & $0.278(0.2$ & $44.094(0.330)$ \\
\hline Gabi & $1.120(0.010)$ & $-0.100(0.1$ & $0.118(0.113)$ & $44.637(0.192)$ \\
\hline Koeke & 1.2 & -0.015( & $0.112(0.1$ & 45.026( \\
\hline Lanc & 1.2 & 0.011 & & 45.109( \\
\hline Manipogo & $0.854(0.010)$ & $0.116(0.1$ & $0.136(0.1$ & $43.897(0.243)$ \\
\hline Mcguire & & & & \\
\hline Ombo & & -0.372( & $0.1 \zeta$ & 44.378 \\
\hline Patuxent & 0.97 & 0.026( & 0.13 & 44.385 \\
\hline Rakke & $0.740(0.010)$ & $-0.001(0.135)$ & $0.184(0.152)$ & $43.368(0.224)$ \\
\hline Spock & $0.839(0.010)$ & $0.058(0.222)$ & $0.158(0.180)$ & $43.429(0.397)$ \\
\hline Strolge & $1.010(0.010)$ & $-0.206(0.166)$ & $0.125(0.127)$ & $44.598(0.216)$ \\
\hline Thames & $0.954(0.010)$ & $0.115(0.2$ & $0.203(0.208)$ & $44.113(0.390)$ \\
\hline Zwicky & $0.521(0.010)$ & $-0.185(0.135)$ & $0.201(0.179)$ & $42.498(0.230)$ \\
\hline anguta & $0.670(0.010)$ & $0.212(0.185)$ & $0.299(0.239)$ & $43.089(0.318)$ \\
\hline aphrodi & & & & $44.848(0.201)$ \\
\hline bilbo & $0.216(0.010)$ & $-0.193(0.139)$ & & $40.333(0.302)$ \\
\hline borg & $1.340(0.010)$ & $-0.224(0.174)$ & $0.467(0.263)$ & $44.875(0.312)$ \\
\hline elvis & $0.840(0.010)$ & $0.110(0.157)$ & $0.194(0.155)$ & $43.626(0.225)$ \\
\hline frodo & $0.735(0.010)$ & $0.258(0.182)$ & $0.213(0.229)$ & $43.088(0.282)$ \\
\hline gilgamesh & $1.551(0.010)$ & $-0.191(0.184)$ & $0.768(0.282)$ & $45.093(0.296)$ \\
\hline inanna & $1.307(0.010)$ & $0.239(0.267)$ & $0.310(0.276)$ & $45.146(0.359)$ \\
\hline isis & $0.526(0.010)$ & $-0.321(0.106)$ & $0.323(0.245)$ & $43.481(0.325)$ \\
\hline mcenroe & $0.900(0.010)$ & $-0.184(0.107)$ & $0.310(0.263)$ & $43.412(0.365)$ \\
\hline nanna & $1.140(0.010)$ & $0.309(0.340)$ & $0.112(0.123)$ & $44.569(0.461)$ \\
\hline
\end{tabular}


Table 3-Continued

\begin{tabular}{|c|c|c|c|c|}
\hline SN Ia & $z_{C M B}$ & $\Delta$ & $A_{V}$ & $\mu^{1}$ \\
\hline qiqirn & $0.640(0.010)$ & $0.121(0.230)$ & $0.294(0.258)$ & $42.799(0.394)$ \\
\hline ramone & $0.954(0.010)$ & $0.142(0.187)$ & $0.136(0.115)$ & $44.187(0.295)$ \\
\hline sasquatch & $1.390(0.010)$ & $0.006(0.311)$ & $0.482(0.281)$ & $44.933(0.280)$ \\
\hline torngasak & $1.265(0.010)$ & $-0.060(0.232)$ & $0.357(0.234)$ & $44.831(0.321)$ \\
\hline vilas & $0.935(0.010)$ & $-0.108(0.130)$ & $0.271(0.256)$ & $43.790(0.381)$ \\
\hline b010 & $0.590(0.003)$ & $-0.170(0.180)$ & $0.119(0.091)$ & $42.966(0.212)$ \\
\hline b013 & $0.427(0.003)$ & $0.016(0.139)$ & $0.204(0.151)$ & $41.949(0.221)$ \\
\hline b020 & $0.426(0.003)$ & $0.055(0.325)$ & $0.228(0.201)$ & $41.742(0.374)$ \\
\hline d033 & $0.530(0.008)$ & $-0.326(0.108)$ & $0.107(0.089)$ & $42.932(0.156)$ \\
\hline $\mathrm{d} 083$ & $0.333(0.001)$ & $-0.284(0.060)$ & $0.103(0.080)$ & $40.691(0.129)$ \\
\hline $\mathrm{d} 084$ & $0.516(0.006)$ & $-0.200(0.205)$ & $0.226(0.190)$ & $42.941(0.286)$ \\
\hline $\mathrm{d} 085$ & $0.401(0.008)$ & $-0.241(0.111)$ & $0.216(0.156)$ & $41.924(0.215)$ \\
\hline $\mathrm{d} 086$ & $0.204(0.003)$ & $-0.177(0.064)$ & $0.663(0.320)$ & $40.030(0.312)$ \\
\hline $\mathrm{d} 087$ & $0.337(0.004)$ & $-0.125(0.093)$ & $0.152(0.122)$ & $41.299(0.192)$ \\
\hline d089 & $0.424(0.003)$ & $-0.212(0.117)$ & $0.162(0.133)$ & $42.023(0.184)$ \\
\hline d093 & $0.361(0.003)$ & $-0.330(0.067)$ & $0.087(0.074)$ & $41.708(0.133)$ \\
\hline d097 & $0.434(0.007)$ & $-0.329(0.067)$ & $0.138(0.111)$ & $42.076(0.161)$ \\
\hline d099 & $0.210(0.002)$ & $-0.120(0.085)$ & $0.141(0.121)$ & $40.401(0.193)$ \\
\hline $\mathrm{d} 117$ & $0.301(0.005)$ & $0.311(0.197)$ & $0.252(0.184)$ & $41.362(0.257)$ \\
\hline d149 & $0.344(0.008)$ & $-0.223(0.088)$ & $0.211(0.149)$ & $41.585(0.203)$ \\
\hline $\mathrm{e} 020$ & $0.159(0.006)$ & $-0.041(0.142)$ & $0.473(0.343)$ & $39.749(0.302)$ \\
\hline e029 & $0.332(0.008)$ & $0.258(0.228)$ & $0.219(0.201)$ & $41.502(0.253)$ \\
\hline e108 & $0.472(0.007)$ & $-0.284(0.123)$ & $0.087(0.073)$ & $42.257(0.145)$ \\
\hline e132 & $0.235(0.006)$ & $-0.131(0.094)$ & $0.810(0.281)$ & $40.347(0.280)$ \\
\hline e136 & $0.348(0.006)$ & $0.323(0.172)$ & $0.338(0.218)$ & $41.580(0.269)$ \\
\hline e138 & $0.611(0.006)$ & $-0.288(0.120)$ & $0.118(0.085)$ & $42.971(0.171)$ \\
\hline e140 & $0.614(0.006)$ & $-0.197(0.143)$ & $0.133(0.087)$ & $42.881(0.166)$ \\
\hline e147 & $0.641(0.008)$ & $-0.184(0.149)$ & $0.084(0.061)$ & $43.002(0.172)$ \\
\hline e148 & $0.429(0.006)$ & $-0.116(0.168)$ & $0.122(0.102)$ & $42.229(0.194)$ \\
\hline e149 & $0.491(0.006)$ & $0.005(0.157)$ & $0.205(0.177)$ & $42.215(0.258)$ \\
\hline f011 & $0.536(0.006)$ & $-0.092(0.237)$ & $0.176(0.132)$ & $42.640(0.238)$ \\
\hline f041 & $0.558(0.007)$ & $-0.300(0.124)$ & $0.103(0.082)$ & $42.695(0.153)$ \\
\hline f076 & $0.410(0.004)$ & $0.187(0.300)$ & $0.261(0.199)$ & $41.426(0.322)$ \\
\hline f216 & $0.595(0.011)$ & $-0.105(0.235)$ & $0.133(0.098)$ & $43.320(0.243)$ \\
\hline $\mathrm{f} 221$ & $0.443(0.004)$ & $-0.047(0.208)$ & $0.108(0.097)$ & $42.834(0.224)$ \\
\hline $\mathrm{f} 231$ & $0.620(0.008)$ & $-0.249(0.143)$ & $0.102(0.074)$ & $43.029(0.161)$ \\
\hline f 235 & $0.422(0.006)$ & $0.168(0.220)$ & $0.128(0.107)$ & $41.777(0.231)$ \\
\hline $\mathrm{f} 244$ & $0.546(0.005)$ & $-0.024(0.257)$ & $0.154(0.121)$ & $42.693(0.251)$ \\
\hline g001 & $0.268(0.002)$ & $0.171(0.126)$ & $0.092(0.078)$ & $40.844(0.155)$ \\
\hline g005 & $0.218(0.007)$ & $-0.260(0.038)$ & $0.445(0.267)$ & $40.349(0.270)$ \\
\hline g052 & $0.384(0.006)$ & $0.383(0.167)$ & $0.135(0.119)$ & $41.566(0.215)$ \\
\hline g055 & $0.302(0.006)$ & $-0.296(0.073)$ & $1.028(0.334)$ & $41.357(0.354)$ \\
\hline g097 & $0.339(0.004)$ & $-0.285(0.100)$ & $0.364(0.266)$ & $41.503(0.302)$ \\
\hline g160 & $0.507(0.019)$ & $-0.317(0.083)$ & $0.192(0.191)$ & $42.390(0.254)$ \\
\hline g240 & $0.689(0.005)$ & $-0.167(0.172)$ & $0.074(0.052)$ & $43.027(0.193)$ \\
\hline g276 & $0.244(0.001)$ & $-0.043(0.066)$ & $0.513(0.309)$ & $40.499(0.327)$ \\
\hline
\end{tabular}


Table 3-Continued

\begin{tabular}{|c|c|c|c|c|}
\hline SN Ia & $z_{C M B}$ & $\Delta$ & $A_{V}$ & $\mu^{1}$ \\
\hline h300 & $0.656(0.011)$ & $-0.281(0.135)$ & $0.086(0.057)$ & $43.082(0.160)$ \\
\hline h319 & $0.478(0.002)$ & $-0.281(0.113)$ & $0.184(0.136)$ & $42.368(0.196)$ \\
\hline h323 & $0.603(0.007)$ & $-0.114(0.185)$ & $0.137(0.098)$ & $42.991(0.215)$ \\
\hline h342 & $0.421(0.005)$ & $-0.365(0.074)$ & $0.107(0.086)$ & $42.156(0.141)$ \\
\hline h363 & $0.211(0.005)$ & $0.055(0.106)$ & $0.859(0.318)$ & $40.251(0.315)$ \\
\hline h364 & $0.344(0.004)$ & $-0.017(0.108)$ & $0.110(0.089)$ & $41.303(0.163)$ \\
\hline $\mathrm{k} 411$ & $0.565(0.003)$ & $-0.131(0.186)$ & $0.141(0.112)$ & $42.442(0.218)$ \\
\hline $\mathrm{k} 425$ & $0.274(0.003)$ & $-0.025(0.195)$ & $0.280(0.250)$ & $41.083(0.280)$ \\
\hline $\mathrm{k} 429$ & $0.171(0.006)$ & $-0.105(0.092)$ & $0.168(0.137)$ & $39.853(0.165)$ \\
\hline $\mathrm{k} 430$ & $0.576(0.007)$ & $-0.110(0.222)$ & $0.130(0.098)$ & $43.288(0.218)$ \\
\hline $\mathrm{k} 441$ & $0.671(0.007)$ & $-0.206(0.181)$ & $0.101(0.063)$ & $43.232(0.180)$ \\
\hline $\mathrm{k} 490$ & $0.709(0.001)$ & $-0.282(0.151)$ & $0.080(0.053)$ & $43.471(0.168)$ \\
\hline $\mathrm{m} 026$ & $0.656(0.006)$ & $-0.204(0.174)$ & $0.101(0.080)$ & $43.021(0.234)$ \\
\hline $\mathrm{m} 027$ & $0.285(0.003)$ & $-0.131(0.134)$ & $0.476(0.273)$ & $41.410(0.297)$ \\
\hline $\mathrm{m} 032$ & $0.155(0.004)$ & $-0.161(0.086)$ & $0.119(0.104)$ & $39.916(0.179)$ \\
\hline m034 & $0.562(0.006)$ & $-0.079(0.220)$ & $0.154(0.119)$ & $42.771(0.243)$ \\
\hline m039 & $0.249(0.003)$ & $-0.223(0.137)$ & $0.493(0.349)$ & $40.794(0.370)$ \\
\hline $\mathrm{m} 158$ & $0.461(0.007)$ & $-0.341(0.110)$ & $0.229(0.204)$ & $42.569(0.274)$ \\
\hline m193 & $0.336(0.006)$ & $-0.139(0.133)$ & $0.143(0.111)$ & $41.261(0.225)$ \\
\hline n263 & $0.368(0.007)$ & $0.063(0.133)$ & $0.126(0.110)$ & $41.496(0.179)$ \\
\hline n278 & $0.308(0.006)$ & $0.016(0.185)$ & $0.197(0.146)$ & $41.127(0.210)$ \\
\hline n285 & $0.531(0.009)$ & $-0.122(0.213)$ & $0.184(0.139)$ & $42.617(0.256)$ \\
\hline n326 & $0.267(0.006)$ & $0.689(0.208)$ & $0.220(0.154)$ & $40.755(0.255)$ \\
\hline n404 & $0.285(0.006)$ & $-0.061(0.191)$ & $0.727(0.358)$ & $40.544(0.321)$ \\
\hline $\mathrm{p} 425$ & $0.456(0.004)$ & $-0.244(0.206)$ & $0.297(0.290)$ & $42.712(0.356)$ \\
\hline p524 & $0.511(0.003)$ & $-0.235(0.144)$ & $0.181(0.133)$ & $42.398(0.211)$ \\
\hline p528 & $0.780(0.009)$ & $-0.310(0.121)$ & $0.048(0.037)$ & $43.752(0.138)$ \\
\hline sn90O & $0.031(0.001)$ & $-0.175(0.065)$ & $0.101(0.067)$ & $35.794(0.156)$ \\
\hline sn90af & $0.050(0.001)$ & $0.506(0.120)$ & $0.211(0.157)$ & $36.646(0.211)$ \\
\hline $\operatorname{sn} 91 \mathrm{U}$ & $0.033(0.001)$ & $-0.122(0.083)$ & $0.410(0.188)$ & $35.615(0.206)$ \\
\hline sn91ag & $0.014(0.001)$ & $-0.165(0.059)$ & $0.085(0.060)$ & $33.991(0.236)$ \\
\hline sn92J & $0.046(0.001)$ & $1.041(0.090)$ & $0.108(0.091)$ & $36.224(0.156)$ \\
\hline $\operatorname{sn} 92 \mathrm{P}$ & $0.026(0.001)$ & $-0.158(0.081)$ & $0.152(0.108)$ & $35.583(0.192)$ \\
\hline sn92ae & $0.075(0.001)$ & $0.021(0.099)$ & $0.229(0.161)$ & $37.716(0.216)$ \\
\hline sn92ag & $0.026(0.001)$ & $0.027(0.077)$ & $0.509(0.132)$ & $35.166(0.203)$ \\
\hline sn92al & $0.014(0.001)$ & $-0.046(0.053)$ & $0.086(0.053)$ & $34.032(0.226)$ \\
\hline sn92aq & $0.101(0.001)$ & $0.123(0.112)$ & $0.065(0.057)$ & $38.731(0.150)$ \\
\hline sn92bc & $0.020(0.001)$ & $-0.194(0.036)$ & $0.018(0.020)$ & $34.850(0.169)$ \\
\hline sn92bg & $0.036(0.001)$ & $-0.042(0.072)$ & $0.132(0.090)$ & $36.153(0.171)$ \\
\hline sn92bh & $0.045(0.001)$ & $-0.105(0.080)$ & $0.270(0.126)$ & $36.874(0.177)$ \\
\hline sn92bk & $0.058(0.001)$ & $0.388(0.181)$ & $0.095(0.075)$ & $37.194(0.218)$ \\
\hline sn92bl & $0.043(0.001)$ & $0.297(0.123)$ & $0.080(0.061)$ & $36.519(0.168)$ \\
\hline sn92bo & $0.018(0.001)$ & $0.599(0.080)$ & $0.146(0.089)$ & $34.713(0.203)$ \\
\hline sn92bp & $0.079(0.001)$ & $0.073(0.092)$ & $0.076(0.055)$ & $37.801(0.139)$ \\
\hline sn92br & $0.088(0.001)$ & $1.011(0.211)$ & $0.139(0.106)$ & $37.746(0.261)$ \\
\hline sn92bs & $0.063(0.001)$ & $-0.093(0.079)$ & $0.220(0.151)$ & $37.628(0.203)$ \\
\hline
\end{tabular}


Table 3-Continued

\begin{tabular}{|c|c|c|c|c|}
\hline SN Ia & $B$ & $\Delta$ & $A_{V}$ & $\mu^{1}$ \\
\hline sn93B & $0.071(0.001)$ & $-0.112(0.073)$ & $0.193(0.114)$ & $37.797(0.172)$ \\
\hline sn93H & $0.025(0.001)$ & $0.875(0.089)$ & $0.135(0.090)$ & $35.145(0.171)$ \\
\hline sn93O & $0.052(0.001)$ & $0.012(0.069)$ & $0.072(0.066)$ & $37.155(0.139)$ \\
\hline sn93ac & $0.049(0.001)$ & $-0.044(0.109)$ & $0.354(0.198)$ & $36.878(0.219)$ \\
\hline sn93ag & $0.050(0.001)$ & $0.051(0.086)$ & $0.177(0.106)$ & $37.087(0.167)$ \\
\hline sn94M & $0.024(0.001)$ & $0.251(0.083)$ & $0.270(0.117)$ & $35.214(0.188)$ \\
\hline sn94S & $0.016(0.001)$ & $-0.098(0.074)$ & $0.061(0.051)$ & $34.357(0.207)$ \\
\hline $\operatorname{sn} 94 \mathrm{~T}$ & $0.036(0.001)$ & $0.702(0.116)$ & $0.122(0.100)$ & $35.999(0.165)$ \\
\hline sn95ac & $0.049(0.001)$ & $-0.238(0.047)$ & $0.224(0.104)$ & $36.535(0.147)$ \\
\hline sn95ak & $0.022(0.001)$ & $0.114(0.066)$ & $0.628(0.117)$ & $34.724(0.197)$ \\
\hline sn95bd & $0.014(0.001)$ & $-0.249(0.044)$ & $0.537(0.261)$ & $33.969(0.236)$ \\
\hline sn96C & $0.028(0.001)$ & $-0.138(0.054)$ & $0.186(0.104)$ & $35.928(0.174)$ \\
\hline sn96ab & $0.124(0.001)$ & $0.132(0.165)$ & $0.163(0.170)$ & $38.887(0.288)$ \\
\hline sn96bl & $0.035(0.001)$ & $-0.128(0.064)$ & $0.248(0.115)$ & $36.059(0.163)$ \\
\hline sn96bo & $0.016(0.001)$ & $0.044(0.070)$ & $0.986(0.120)$ & $34.004(0.224)$ \\
\hline sn96bv & $0.017(0.001)$ & $-0.223(0.063)$ & $0.588(0.153)$ & $34.180(0.239)$ \\
\hline sn97E & $0.013(0.001)$ & $0.321(0.085)$ & $0.278(0.109)$ & $34.055(0.240)$ \\
\hline $\operatorname{sn} 97 \mathrm{Y}$ & $0.017(0.001)$ & $0.042(0.075)$ & $0.269(0.090)$ & $34.504(0.214)$ \\
\hline $\mathrm{sn} 97 \mathrm{cn}$ & $0.017(0.001)$ & $1.410(0.046)$ & $0.100(0.068)$ & $34.547(0.196)$ \\
\hline sn97dg & $0.030(0.001)$ & $-0.010(0.076)$ & $0.235(0.109)$ & $36.115(0.173)$ \\
\hline sn97do & $0.011(0.001)$ & $-0.162(0.081)$ & $0.371(0.102)$ & $33.560(0.297)$ \\
\hline sn98V & $0.017(0.001)$ & $-0.067(0.062)$ & $0.270(0.121)$ & $34.342(0.207)$ \\
\hline sn98ab & $0.028(0.001)$ & $-0.128(0.051)$ & $0.453(0.081)$ & $35.165(0.164)$ \\
\hline sn98bp & $0.010(0.001)$ & $1.142(0.055)$ & $0.188(0.101)$ & $33.269(0.288)$ \\
\hline sn98co & $0.017(0.001)$ & $0.443(0.194)$ & $0.365(0.177)$ & $34.372(0.223)$ \\
\hline sn98de & $0.016(0.001)$ & $1.451(0.035)$ & $0.337(0.104)$ & $34.449(0.213)$ \\
\hline $\operatorname{sn} 98 d x$ & $0.054(0.001)$ & $0.200(0.099)$ & $0.107(0.066)$ & $36.885(0.140)$ \\
\hline sn98ef & $0.017(0.001)$ & $0.119(0.096)$ & $0.181(0.097)$ & $34.170(0.213)$ \\
\hline sn98eg & $0.024(0.001)$ & $0.001(0.115)$ & $0.277(0.123)$ & $35.305(0.187)$ \\
\hline sn99aa & $0.015(0.001)$ & $-0.277(0.028)$ & $0.024(0.025)$ & $34.462(0.203)$ \\
\hline sn99aw & $0.039(0.001)$ & $-0.384(0.032)$ & $0.019(0.020)$ & $36.544(0.116)$ \\
\hline sn99cc & $0.032(0.001)$ & 0.337(0.099) & $0.195(0.098)$ & $35.811(0.151)$ \\
\hline sn99cp & $0.010(0.001)$ & $-0.111(0.099)$ & $0.099(0.065)$ & $33.500(0.291)$ \\
\hline sn99da & $0.013(0.001)$ & $1.447(0.037)$ & $0.218(0.107)$ & $33.913(0.253)$ \\
\hline sn99dk & $0.014(0.001)$ & $-0.251(0.048)$ & $0.266(0.100)$ & $34.215(0.238)$ \\
\hline sn99dq & $0.013(0.001)$ & $-0.288(0.029)$ & $0.378(0.089)$ & $33.662(0.232)$ \\
\hline sn99ee & $0.011(0.001)$ & $-0.211(0.030)$ & $0.930(0.069)$ & $33.343(0.279)$ \\
\hline sn99ef & $0.038(0.001)$ & $-0.098(0.079)$ & $0.063(0.055)$ & $36.666(0.158)$ \\
\hline sn99ej & $0.013(0.001)$ & $0.367(0.112)$ & $0.151(0.090)$ & $34.404(0.259)$ \\
\hline sn99ek & $0.018(0.001)$ & $0.042(0.062)$ & $0.498(0.277)$ & $34.317(0.206)$ \\
\hline sn99gd & $0.019(0.001)$ & $-0.004(0.073)$ & $1.452(0.112)$ & $34.526(0.209)$ \\
\hline sn99gp & $0.026(0.001)$ & $-0.351(0.028)$ & $0.109(0.064)$ & $35.610(0.151)$ \\
\hline sn00bh & $0.024(0.001)$ & $-0.048(0.066)$ & $0.170(0.105)$ & $35.276(0.200)$ \\
\hline sn00ca & $0.025(0.001)$ & $-0.138(0.048)$ & $0.025(0.029)$ & $35.257(0.153)$ \\
\hline sn00cf & $0.036(0.001)$ & $-0.006(0.066)$ & $0.238(0.107)$ & $36.330(0.159)$ \\
\hline sn00cn & $0.023(0.001)$ & $0.733(0.076)$ & $0.200(0.115)$ & $35.099(0.170)$ \\
\hline
\end{tabular}


Table 3-Continued

\begin{tabular}{|c|c|c|c|c|}
\hline SN Ia & $B$ & $\Delta$ & $A_{V}$ & $\mu^{1}$ \\
\hline sn00dk & $0.016(0.001)$ & $0.594(0.069)$ & $0.037(0.039)$ & $34.375(0.203)$ \\
\hline sn00fa & $0.022(0.001)$ & $-0.124(0.062)$ & $0.468(0.098)$ & $34.925(0.195)$ \\
\hline $\mathrm{sn} 01 \mathrm{G}$ & $0.017(0.001)$ & $-0.129(0.069)$ & $0.063(0.055)$ & $34.524(0.198)$ \\
\hline sn01ah & $0.058(0.001)$ & $-0.223(0.111)$ & $0.062(0.048)$ & $37.263(0.159)$ \\
\hline sn01az & $0.041(0.001)$ & $-0.030(0.104)$ & $0.088(0.072)$ & $36.472(0.149)$ \\
\hline sn01ba & $0.030(0.001)$ & $-0.119(0.054)$ & $0.041(0.041)$ & $35.899(0.143)$ \\
\hline sn01bf & $0.015(0.001)$ & $-0.173(0.074)$ & $0.269(0.113)$ & $34.044(0.228)$ \\
\hline sn01bt & $0.014(0.001)$ & $0.059(0.052)$ & $0.676(0.103)$ & $33.900(0.233)$ \\
\hline sn01cn & $0.015(0.001)$ & $-0.014(0.051)$ & $0.506(0.098)$ & $34.082(0.223)$ \\
\hline sn01cp & $0.022(0.001)$ & $0.054(0.177)$ & $0.100(0.076)$ & $35.003(0.242)$ \\
\hline $\mathrm{sn} 01 \mathrm{cz}$ & $0.016(0.001)$ & $-0.091(0.053)$ & $0.307(0.116)$ & $34.252(0.219)$ \\
\hline sn01da & $0.016(0.001)$ & $-0.038(0.090)$ & $0.219(0.129)$ & $34.562(0.237)$ \\
\hline sn01eh & $0.036(0.001)$ & $-0.275(0.041)$ & $0.028(0.027)$ & $36.295(0.124)$ \\
\hline sn01en & $0.015(0.001)$ & $0.065(0.072)$ & $0.200(0.093)$ & $34.249(0.227)$ \\
\hline sn01ep & $0.013(0.001)$ & $0.081(0.068)$ & $0.474(0.085)$ & $33.795(0.246)$ \\
\hline sn01fe & $0.014(0.001)$ & $-0.141(0.054)$ & $0.168(0.086)$ & $34.124(0.234)$ \\
\hline sn01fh & $0.011(0.001)$ & $0.583(0.111)$ & $0.150(0.126)$ & $33.728(0.272)$ \\
\hline sn01ie & $0.031(0.001)$ & $-0.014(0.095)$ & $0.333(0.155)$ & $35.760(0.213)$ \\
\hline $\operatorname{sn} 01 n$ & $0.022(0.001)$ & $-0.026(0.092)$ & $0.699(0.168)$ & $35.002(0.223)$ \\
\hline $\mathrm{sn} 01 \mathrm{v}$ & $0.016(0.001)$ & $-0.287(0.032)$ & $0.203(0.070)$ & $34.082(0.205)$ \\
\hline sn02bf & $0.025(0.001)$ & $-0.147(0.064)$ & $0.337(0.136)$ & $35.433(0.196)$ \\
\hline sn02ck & $0.030(0.001)$ & $-0.058(0.073)$ & $0.139(0.089)$ & $35.771(0.169)$ \\
\hline $\mathrm{sn} 02 \mathrm{cr}$ & $0.010(0.001)$ & $-0.027(0.061)$ & $0.270(0.097)$ & $33.506(0.292)$ \\
\hline sn02de & $0.028(0.001)$ & $-0.154(0.210)$ & $0.506(0.124)$ & $35.631(0.193)$ \\
\hline sn02dj & $0.010(0.001)$ & $-0.204(0.114)$ & $0.415(0.114)$ & $33.127(0.296)$ \\
\hline sn02do & $0.015(0.001)$ & $0.739(0.119)$ & $0.140(0.104)$ & $34.305(0.223)$ \\
\hline sn02dp & $0.011(0.001)$ & $0.030(0.140)$ & $0.472(0.124)$ & $33.505(0.293)$ \\
\hline sn02es & $0.018(0.001)$ & $0.326(0.151)$ & $0.676(0.188)$ & $34.415(0.235)$ \\
\hline $\mathrm{sn} 02 \mathrm{fb}$ & $0.015(0.001)$ & $1.278(0.085)$ & $0.687(0.153)$ & $34.209(0.230)$ \\
\hline sn02g & $0.035(0.001)$ & $0.338(0.381)$ & $0.884(0.258)$ & $35.663(0.408)$ \\
\hline sn02ha & $0.013(0.001)$ & $0.196(0.089)$ & $0.106(0.082)$ & $34.032(0.239)$ \\
\hline sn02hd & $0.036(0.001)$ & $0.131(0.143)$ & $0.235(0.144)$ & $35.820(0.202)$ \\
\hline sn02he & $0.025(0.001)$ & $0.493(0.114)$ & $0.072(0.061)$ & $35.286(0.196)$ \\
\hline sn02hu & $0.038(0.001)$ & $-0.193(0.051)$ & $0.056(0.047)$ & $36.289(0.128)$ \\
\hline sn02hw & $0.016(0.001)$ & $0.741(0.095)$ & $1.031(0.159)$ & $34.037(0.216)$ \\
\hline sn02jy & $0.020(0.001)$ & $-0.197(0.068)$ & $0.108(0.076)$ & $35.275(0.184)$ \\
\hline sn $02 \mathrm{kf}$ & $0.019(0.001)$ & $0.158(0.068)$ & $0.080(0.057)$ & $35.025(0.186)$ \\
\hline sn03D & $0.024(0.001)$ & $1.101(0.149)$ & $0.540(0.228)$ & $34.883(0.220)$ \\
\hline $\mathrm{sn} 03 \mathrm{U}$ & $0.028(0.001)$ & $0.464(0.093)$ & $0.118(0.081)$ & $35.527(0.164)$ \\
\hline sn03W & $0.021(0.001)$ & $-0.086(0.047)$ & $0.457(0.083)$ & $34.884(0.179)$ \\
\hline sn03ch & $0.030(0.001)$ & $0.160(0.107)$ & $0.102(0.076)$ & $35.970(0.161)$ \\
\hline sn03cq & $0.034(0.001)$ & $0.000(0.090)$ & $0.689(0.262)$ & $35.910(0.288)$ \\
\hline sn03fa & $0.039(0.001)$ & $-0.272(0.039)$ & $0.032(0.028)$ & $36.372(0.119)$ \\
\hline sn03hu & $0.075(0.001)$ & $-0.254(0.081)$ & $0.282(0.167)$ & $37.691(0.195)$ \\
\hline sn03ic & $0.054(0.001)$ & $0.248(0.130)$ & $0.152(0.116)$ & $36.763(0.170)$ \\
\hline sn03it & $0.024(0.001)$ & $0.401(0.110)$ & $0.252(0.120)$ & $35.248(0.188)$ \\
\hline
\end{tabular}


Table 3-Continued

\begin{tabular}{|c|c|c|c|c|}
\hline SN Ia & $B$ & $\Delta$ & $A_{V}$ & $\mu^{1}$ \\
\hline sn03iv & $0.034(0.001)$ & $0.403(0.106)$ & $0.074(0.055)$ & $36.213(0.155)$ \\
\hline sn04as & $0.032(0.001)$ & $-0.154(0.057)$ & $0.378(0.101)$ & $36.155(0.164)$ \\
\hline sn04bg & $0.022(0.001)$ & $-0.124(0.060)$ & $0.097(0.077)$ & $35.142(0.180)$ \\
\hline sn04l & $0.033(0.001)$ & $0.044(0.115)$ & $0.694(0.146)$ & $35.901(0.201)$ \\
\hline $\mathrm{sn} 05 \mathrm{dv}$ & $0.011(0.001)$ & $1.229(0.136)$ & $0.918(0.345)$ & $32.994(0.332)$ \\
\hline sn05el & $0.015(0.001)$ & $0.259(0.057)$ & $0.040(0.034)$ & $34.286(0.218)$ \\
\hline sn05eq & $0.028(0.001)$ & $-0.266(0.039)$ & $0.080(0.063)$ & $35.886(0.148)$ \\
\hline sn05eu & $0.034(0.001)$ & $-0.251(0.042)$ & $0.061(0.048)$ & $36.180(0.140)$ \\
\hline sn05hc & $0.045(0.001)$ & $-0.129(0.056)$ & $0.132(0.081)$ & $36.869(0.140)$ \\
\hline sn05hf & $0.042(0.001)$ & $0.198(0.096)$ & $0.089(0.084)$ & $36.564(0.158)$ \\
\hline sn05hj & $0.057(0.001)$ & $-0.314(0.061)$ & $0.094(0.070)$ & $37.372(0.129)$ \\
\hline sn05iq & $0.033(0.001)$ & $0.172(0.074)$ & $0.086(0.068)$ & $36.203(0.148)$ \\
\hline sn05ir & $0.075(0.001)$ & $-0.370(0.078)$ & $0.107(0.083)$ & $38.110(0.138)$ \\
\hline sn05kc & $0.014(0.001)$ & $0.009(0.064)$ & $1.137(0.122)$ & $33.767(0.242)$ \\
\hline sn05ki & $0.021(0.001)$ & $0.376(0.068)$ & $0.084(0.056)$ & $34.806(0.179)$ \\
\hline sn05ls & $0.021(0.001)$ & $-0.236(0.057)$ & $1.153(0.125)$ & $34.411(0.205)$ \\
\hline sn05lz & $0.040(0.001)$ & $0.203(0.088)$ & $0.394(0.118)$ & $36.519(0.166)$ \\
\hline $\mathrm{sn} 05 \mathrm{mc}$ & $0.026(0.001)$ & $0.888(0.080)$ & $0.367(0.151)$ & $35.361(0.170)$ \\
\hline $\mathrm{sn} 05 \mathrm{~ms}$ & $0.026(0.001)$ & $-0.134(0.045)$ & $0.118(0.067)$ & $35.674(0.157)$ \\
\hline $\mathrm{sn} 05 \mathrm{mz}$ & $0.017(0.001)$ & $1.213(0.058)$ & $0.850(0.157)$ & $34.077(0.224)$ \\
\hline sn05na & $0.027(0.001)$ & $-0.007(0.055)$ & $0.120(0.084)$ & $35.462(0.165)$ \\
\hline sn06ac & $0.024(0.001)$ & $0.175(0.066)$ & $0.209(0.082)$ & $35.292(0.173)$ \\
\hline sn06ak & $0.039(0.001)$ & $0.194(0.106)$ & $0.105(0$. & $36.375(0.178)$ \\
\hline sn06al & $0.069(0.001)$ & $0.164(0.101)$ & $0.075(0.056)$ & $37.834(0.134)$ \\
\hline sn06an & $0.065(0.001)$ & $-0.167(0.073)$ & $0.070(0.055)$ & $37.695(0.123)$ \\
\hline sn06ar & $0.023(0.001)$ & $0.258(0.394)$ & $0.392(0.209)$ & $35.249(0.278)$ \\
\hline $\operatorname{sn} 06 a x$ & $0.018(0.001)$ & $-0.141(0.040)$ & $0.062(0.046)$ & $34.684(0.186)$ \\
\hline sn06az & $0.032(0.001)$ & $0.248(0.054)$ & $0.041(0.034)$ & $35.852(0.137)$ \\
\hline sn06bd & $0.026(0.001)$ & $1.492(0.097)$ & $0.297(0.182)$ & $35.586(0.203)$ \\
\hline sn06bq & $0.021(0.001)$ & $0.286(0.071)$ & $0.235(0.111)$ & $35.163(0.186)$ \\
\hline sn06bt & $0.033(0.001)$ & $-0.198(0.047)$ & $0.664(0.098)$ & $35.784(0.158)$ \\
\hline sn06bu & $0.084(0.001)$ & $-0.270(0.102)$ & $0.067(0.065)$ & $38.017(0.131)$ \\
\hline sn06bw & $0.031(0.001)$ & $0.427(0.132)$ & $0.358(0.215)$ & $35.682(0.206)$ \\
\hline sn06bz & $0.028(0.001)$ & $1.505(0.075)$ & $0.351(0.193)$ & $35.307(0.191)$ \\
\hline sn06cc & $0.033(0.001)$ & $-0.173(0.046)$ & $1.316(0.092)$ & $35.777(0.156)$ \\
\hline sn06cf & $0.042(0.001)$ & $-0.056(0.095)$ & $0.114(0.080)$ & $36.564(0.146)$ \\
\hline sn06cg & $0.029(0.001)$ & $0.566(0.180)$ & $0.243(0.179)$ & $35.181(0.212)$ \\
\hline sn06cj & $0.068(0.001)$ & $-0.147(0.119)$ & $0.045(0.037)$ & $37.822(0.131)$ \\
\hline sn06cp & $0.023(0.001)$ & $-0.138(0.061)$ & $0.704(0.109)$ & $34.846(0.189)$ \\
\hline sn06cq & $0.049(0.001)$ & $-0.234(0.104)$ & $0.162(0.120)$ & $37.069(0.175)$ \\
\hline sn06cs & $0.024(0.001)$ & $1.521(0.098)$ & $0.441(0.301)$ & $35.197(0.244)$ \\
\hline sn06ef & $0.017(0.001)$ & $0.099(0.080)$ & $0.119(0.086)$ & $34.841(0.225)$ \\
\hline sn06ej & $0.019(0.001)$ & $0.190(0.078)$ & $0.095(0.067)$ & $34.974(0.196)$ \\
\hline sn06em & $0.018(0.001)$ & $1.463(0.083)$ & $0.497(0.237)$ & $35.063(0.247)$ \\
\hline sn06en & $0.031(0.001)$ & $-0.043(0.063)$ & $0.326(0.153)$ & $35.930(0.200)$ \\
\hline sn06et & $0.021(0.001)$ & $-0.225(0.074)$ & $0.474(0.139)$ & $35.032(0.217)$ \\
\hline
\end{tabular}


Table 3-Continued

\begin{tabular}{|c|c|c|c|c|}
\hline SN Ia & $z_{C M B}$ & $\Delta$ & $A_{V}$ & $\mu^{1}$ \\
\hline sn06gj & $0.028(0.001)$ & $0.513(0.135)$ & $1.112(0$ & $35.306(0.181)$ \\
\hline sn06gr & $0.034(0.001)$ & $-0.254(0.036)$ & $.353(0.098)$ & $6.209(0.153)$ \\
\hline sn06gt & $0.044(0.001)$ & $0.707(0.151)$ & $0.147(0.131)$ & $36.796(0.180)$ \\
\hline sn06hc & $0.013(0.001)$ & $1.314(0.063)$ & $0.442(0.207)$ & $34.230(0.254)$ \\
\hline sn06hb & $0.015(0.001)$ & $0.559(0.089)$ & $0.158(0.106)$ & $34.212(0.225)$ \\
\hline sn06is & $0.031(0.002)$ & $-0.335(0.047)$ & $0.029(0.025)$ & $35.932(0.153)$ \\
\hline sn06kf & $0.021(0.001)$ & $0.661(0.092)$ & $0.070(0.056)$ & $34.959(0.193)$ \\
\hline sn06le & $0.017(0.001)$ & $-0.232(0.033)$ & $0.087(0.081)$ & $34.713(0.198)$ \\
\hline sn06lf & $0.013(0.001)$ & $0.263(0.062)$ & $0.193(0.153)$ & $33.721(0.254)$ \\
\hline sn06mo & $0.036(0.001)$ & $0.667(\mathrm{C}$ & $0.112(0$ & 36.18 \\
\hline $\mathrm{sn} 06 \mathrm{mp}$ & $0.023(0.001)$ & $-0.128(0$ & $0.303(0.121)$ & $35.264(0.199)$ \\
\hline sn06n & $0.014(\mathrm{C}$ & $0.458(\mathrm{C}$ & $0.120(C$ & $34.168(0.227)$ \\
\hline sn06nz & $0.037(0.001)$ & $1.101(0$ & 02144 & $36.044(0.189)$ \\
\hline sn06oa & 0.05 & -0.2 & $0.1^{\prime}$ & 37.34 \\
\hline sn06ob & 0.0 & 0.658 & 0.066 & $37.11:$ \\
\hline sn06on & 0.0 & -0.084 & 0.413 & $37.46^{\prime}$ \\
\hline sn06os & 0.03 & -0.072( & & \\
\hline sn06qo & 0.030 & -0.163( & 0.745 & 35.528 \\
\hline sn06s & 0.033 & $-0.201(C$ & $0.317(0$ & $36.126(0$. \\
\hline sn06sr & 0.02 & $0.1^{\prime}$ & 0.2 & 35.282 \\
\hline sn06td & 0.01 & & 0.4 & 34.425 \\
\hline sn06te & 0.03 & -0.0 & $0097-3-2$ & 36.052 \\
\hline sn07ae & $0.064(0.001)$ & $-0.203(0.0$ & $0.106(0.0$ & $37.336(0.137)$ \\
\hline & & -0 . & & 35. \\
\hline sn07al & 0.01 & 1.334 & 0.6 & 34.153 \\
\hline sn07ap & $0.016(0.001)$ & $0.429(0.094)$ & $0.040(0.0$ & $34.573(0.222)$ \\
\hline sn07au & $0.020(0.001)$ & $1.060(0.059)$ & $0.191(0.120)$ & $34.671(0.193)$ \\
\hline & & & & $35.910(0.157)$ \\
\hline sno & & 0.242 & 0.3 & 34.916 \\
\hline sn07bd & 0.03 & 0.2 & 0.0 & 35. \\
\hline sn07bz & $0.023(0.001)$ & $-0.079(0.078)$ & $0.284(0.141)$ & $35.830(0.205)$ \\
\hline sn07ca & $0.015(0.001)$ & $-0.184(0.047)$ & & $34.394(0.238)$ \\
\hline $\mathrm{sn} 07 \mathrm{ci}$ & & & & 34.285 \\
\hline $\mathrm{sn} 07 \mathrm{co}$ & $0.027(0.001)$ & $-0.052(0.064)$ & $0.578(0.113)$ & $35.379(0.168)$ \\
\hline $\mathrm{sn} 07 \mathrm{cq}$ & $0.025(0.001)$ & $0.047(0.064)$ & $0.178(0.109)$ & $35.164(0.183)$ \\
\hline sn07f & $0.024(0.001)$ & $-0.141(0.040)$ & $0.097(0.070)$ & $35.440(0.164)$ \\
\hline $\operatorname{sn} 07 \mathrm{n}$ & & & & \\
\hline sn07o & $0.036(0.001)$ & $0.012(0.064)$ & $0.080(0.066)$ & $36.225(0.147)$ \\
\hline sn07qe & $0.023(0.001)$ & $-0.228(0.037)$ & $0.159(0.087)$ & $35.527(0.169)$ \\
\hline $\mathrm{sn} 07 \mathrm{r}$ & $0.031(0.001)$ & $0.204(0.075)$ & $0.074(0.056)$ & $35.968(0.155)$ \\
\hline $\mathrm{sn} 07 \mathrm{~s}$ & $0.015(0.001)$ & $-0.260(0.036)$ & $1.271(0.098)$ & $33.843(0.240)$ \\
\hline sn08L & $0.019(0.001)$ & $0.297(0.162)$ & $0.097(0.079)$ & $34.477(0.245)$ \\
\hline sn08af & $0.034(0.001)$ & $0.249(0.091)$ & $0.092(0.079)$ & $36.095(0.166)$ \\
\hline sn08bf & $0.026(0.001)$ & $-0.142(0.053)$ & $0.116(0.069)$ & $35.250(0.160)$ \\
\hline
\end{tabular}

${ }^{1}$ Distance moduli were calculated with $\mathrm{H}_{\mathrm{o}}=65 \mathrm{kms}^{-1}$ and $M_{V}=$ -19.504 . Uncertainty of $\mu$ includes $0.078 \mathrm{mag}$ "intrinsic", $\sigma_{v-\text { pec }}(400$ $\mathrm{km} \mathrm{s}^{-1}$ ), and $\sigma_{z}$. 
Table 4. MLCS17 Light Curve Fit Parameters.

\begin{tabular}{|c|c|c|c|c|}
\hline SN Ia & $z_{C M B}$ & $\Delta$ & $A_{V}$ & $\mu^{1}$ \\
\hline 03D1au & $0.504(0.001)$ & $-0.346(0.076)$ & $0.108(0.089)$ & $42.514(0.173)$ \\
\hline 03D1aw & $0.582(0.001)$ & $-0.260(0.139)$ & $0.098(0.082)$ & $43.054(0.194)$ \\
\hline 03D1ax & $0.496(0.001)$ & $0.170(0.119)$ & $0.065(0.056)$ & $42.194(0.180)$ \\
\hline 03D1bp & $0.346(0.001)$ & $0.163(0.152)$ & $0.158(0.104)$ & $41.459(0.208)$ \\
\hline 03D1co & $0.679(0.001)$ & $-0.313(0.121)$ & $0.151(0.126)$ & $43.575(0.256)$ \\
\hline 03D1ew & $0.868(0.001)$ & $-0.255(0.120)$ & $0.111(0.084)$ & $43.794(0.207)$ \\
\hline 03D1fc & $0.331(0.001)$ & $-0.170(0.147)$ & $0.123(0.078)$ & $41.242(0.181)$ \\
\hline 03D1fl & $0.688(0.001)$ & $-0.264(0.132)$ & $0.130(0.101)$ & $43.075(0.211)$ \\
\hline 03D1fq & $0.800(0.001)$ & $0.054(0.182)$ & $0.095(0.079)$ & $43.764(0.259)$ \\
\hline 03D1gt & $0.548(0.001)$ & $0.351(0.292)$ & $0.497(0.279)$ & $42.416(0.337)$ \\
\hline 03D3af & $0.532(0.001)$ & $-0.083(0.163)$ & $0.112(0.098)$ & $42.846(0.241)$ \\
\hline 03D3aw & $0.449(0.001)$ & $-0.112(0.132)$ & $0.058(0.041)$ & $42.059(0.207)$ \\
\hline 03D3ay & $0.371(0.001)$ & $-0.237(0.120)$ & $0.051(0.037)$ & $41.844(0.194)$ \\
\hline 03D3ba & $0.291(0.001)$ & $-0.140(0.117)$ & $0.408(0.162)$ & $40.889(0.235)$ \\
\hline 03D3cc & $0.463(0.001)$ & $-0.252(0.097)$ & $0.096(0.065)$ & $42.203(0.169)$ \\
\hline 03D3cd & $0.461(0.001)$ & $-0.249(0.126)$ & $0.071(0.059)$ & $42.053(0.165)$ \\
\hline 03D4ag & $0.285(0.001)$ & $-0.329(0.074)$ & $0.038(0.030)$ & $40.931(0.138)$ \\
\hline 03D4at & $0.633(0.001)$ & $-0.191(0.148)$ & $0.108(0.099)$ & $43.205(0.222)$ \\
\hline $03 \mathrm{D} 4 \mathrm{cx}$ & $0.949(0.001)$ & $-0.274(0.136)$ & $0.054(0.046)$ & $44.157(0.183)$ \\
\hline 03D4cy & $0.927(0.001)$ & $-0.227(0.146)$ & $0.063(0.051)$ & $44.044(0.203)$ \\
\hline $03 \mathrm{D} 4 \mathrm{cz}$ & $0.695(0.001)$ & $0.381(0.200)$ & $0.085(0.087)$ & $42.983(0.309)$ \\
\hline 03D4dh & $0.627(0.001)$ & $-0.314(0.087)$ & $0.130(0.110)$ & $42.908(0.204)$ \\
\hline 03D4di & $0.905(0.001)$ & $-0.342(0.098)$ & $0.074(0.063)$ & $43.821(0.177)$ \\
\hline 03D4dy & $0.604(0.001)$ & $-0.370(0.075)$ & $0.285(0.191)$ & $42.623(0.294)$ \\
\hline 03D4fd & $0.791(0.001)$ & $-0.196(0.152)$ & $0.110(0.103)$ & $43.635(0.234)$ \\
\hline 03D4gf & $0.581(0.001)$ & $-0.162(0.149)$ & $0.043(0.034)$ & $42.864(0.179)$ \\
\hline 03D4gg & $0.592(0.001)$ & $-0.162(0.181)$ & $0.096(0.077)$ & $42.833(0.225)$ \\
\hline 03D4gl & $0.571(0.001)$ & $0.030(0.327)$ & $0.095(0.089)$ & $42.450(0.386)$ \\
\hline 04D1ag & $0.557(0.001)$ & $-0.155(0.186)$ & $0.060(0.057)$ & $42.514(0.212)$ \\
\hline 04D1aj & $0.721(0.001)$ & $-0.331(0.109)$ & $0.123(0.113)$ & $43.419(0.224)$ \\
\hline 04D1ak & $0.526(0.001)$ & $0.483(0.188)$ & $0.111(0.098)$ & $42.382(0.265)$ \\
\hline 04D2cf & $0.369(0.001)$ & $-0.001(0.135)$ & $0.071(0.059)$ & $41.776(0.171)$ \\
\hline 04D2fp & $0.415(0.001)$ & $-0.162(0.094)$ & $0.103(0.067)$ & $41.978(0.152)$ \\
\hline $04 \mathrm{D} 2 \mathrm{fs}$ & $0.357(0.001)$ & $-0.123(0.110)$ & $0.222(0.107)$ & $41.624(0.165)$ \\
\hline 04D2gb & $0.430(0.001)$ & $0.497(0.111)$ & $0.058(0.043)$ & $41.714(0.172)$ \\
\hline 04D2gc & $0.521(0.001)$ & $-0.247(0.109)$ & $0.539(0.177)$ & $42.117(0.284)$ \\
\hline 04D2gp & $0.707(0.001)$ & $0.050(0.243)$ & $0.100(0.103)$ & $43.398(0.315)$ \\
\hline 04D2iu & $0.691(0.001)$ & $0.309(0.226)$ & $0.124(0.115)$ & $43.219(0.347)$ \\
\hline 04D2ja & $0.741(0.001)$ & $-0.175(0.161)$ & $0.087(0.078)$ & $43.559(0.235)$ \\
\hline 04D3co & $0.620(0.001)$ & $-0.089(0.164)$ & $0.096(0.086)$ & $43.234(0.239)$ \\
\hline 04D3cp & $0.830(0.001)$ & $-0.379(0.077)$ & $0.067(0.058)$ & $43.663(0.159)$ \\
\hline 04D3cy & $0.643(0.001)$ & $-0.350(0.094)$ & $0.111(0.091)$ & $43.423(0.191)$ \\
\hline 04D3dd & $1.010(0.001)$ & $-0.320(0.121)$ & $0.042(0.032)$ & $44.678(0.194)$ \\
\hline 04D3df & $0.470(0.001)$ & $0.743(0.119)$ & $0.096(0.070)$ & $41.947(0.181)$ \\
\hline 04D3do & $0.610(0.001)$ & $0.189(0.153)$ & $0.091(0.083)$ & $42.722(0.231)$ \\
\hline 04D3ez & $0.263(0.001)$ & $0.010(0.103)$ & $0.228(0.090)$ & $40.764(0.165)$ \\
\hline
\end{tabular}


Table 4-Continued

\begin{tabular}{|c|c|c|c|c|}
\hline SN Ia & $z_{C M B}$ & $\Delta$ & $A_{V}$ & $\mu^{1}$ \\
\hline 04D3fk & $0.358(0.001)$ & $-0.160(0.092)$ & $0.385(0.086)$ & $41.581(0.155)$ \\
\hline 04D3fq & $730(0.001)$ & $-0.023(0.173)$ & $094(0.084)$ & $43.434(0.245)$ \\
\hline 04D3gt & $0.451(0.001)$ & $-0.088(0.099)$ & $0.635(0.097)$ & $41.797(0.170)$ \\
\hline 04D3gx & $0.910(0.001)$ & $-0.250(0.126)$ & $0.069(0.052)$ & $44.169(0.187)$ \\
\hline 04D3hn & $0.552(0.001)$ & $0.099(0.153)$ & $0.381(0.173)$ & $42.258(0.291)$ \\
\hline 04D3is & $0.710(0.001)$ & $-0.182(0.1$ & & \\
\hline 04D3ki & $0.930(0.001)$ & $-0.071(0.167)$ & $0.070(0.060)$ & $44.202(0.235)$ \\
\hline 04D3kr & $0.337(0.001)$ & $-0.322(0.067)$ & $0.164(0.082)$ & $41.386(0.142)$ \\
\hline 04D3ks & $0.752(0.001)$ & $-0.280(0.120)$ & $0.134(0.118)$ & $43.324(0.241)$ \\
\hline 04D3lu & $0.822(0.001)$ & $-0.186(0.133)$ & $0.092(0.086)$ & $43.794(0.216)$ \\
\hline 04D3ml & $0.950(0.001)$ & $-0.344(0.112)$ & $0.054(0.046)$ & $44.117(0.170)$ \\
\hline 04D3nc & $0.817(0.001)$ & $-0.289(0.120)$ & $0.122(0.090)$ & $43.696(0.211)$ \\
\hline 04D3nh & $0.340(0.001)$ & $-0.248(0.093)$ & $0.132(0.082)$ & $41.566(0.148)$ \\
\hline 04D3nr & $0.960(0.001)$ & $-0.255(0.146)$ & $0.049(0.044)$ & $44.291(0.224)$ \\
\hline 04D3ny & 0.810 & $-0.022(0.1$ & $0.147(0$ & 43.461 \\
\hline 04D3oe & 0.756 & $-0.202(c$ & 0.075( & 43.58 \\
\hline 04D4an & $0.61:$ & & 0.155 ( & 42.913 \\
\hline 04D4bk & $.001)$ & -0.310( & $0.067(0$. & 43.877 \\
\hline 04D4bq & $0.550(0.0$ & -0.230( & $0.178(0$ & 42.690 \\
\hline 04D4dm & 0.81 & $-0.104(0.1$ & 0.143( & 43.670 \\
\hline $04 \mathrm{D} 4 \mathrm{dw}$ & 0.96 & $-0.260(0.143)$ & $0.049(0.0$ & 44.166 \\
\hline $2002 \mathrm{dc}$ & $0.475(0.010)$ & $0.172(0.220)$ & $0.153(0.163)$ & $42.248(0.287)$ \\
\hline $2002 \mathrm{dd}$ & $0.950(0.010)$ & $-0.244(0.1$ & $0.212(0.1$ & $43.969(0.258)$ \\
\hline Eagle & $1.020(0.010)$ & $-0.182(0.147)$ & $0.122(0.1$ & $44.385(0.211)$ \\
\hline Ferguson & 1.0 & 0.071 & $0.175(c$ & 44.025 \\
\hline Gabi & 1.1 & -0.200 & 0.106 & \\
\hline Greenb & $1.140(0.010)$ & $-0.394(0.0$ & $0.085(0.0$ & $0.159)$ \\
\hline Hawk & & & & \\
\hline Koek & & & $0.076(0$ & 44.990 \\
\hline Lanc & 1.2 & 0.157( & 0.161 & $45.083(0$ \\
\hline Manipogo & $0.854(0.010)$ & $0.093(0.183)$ & $0.098(0.086)$ & $43.757(0.246)$ \\
\hline Mcguire & $1.370(0.010)$ & $-0.317(0.102)$ & $0.095(0.080)$ & $45.187(0.185)$ \\
\hline Ombo & & $-0.392(0.093)$ & $0.221(0.142)$ & $44.273(0.213)$ \\
\hline Patuxent & $0.970(0.010)$ & $-0.128(0.215)$ & $0.098(0.097)$ & $44.432(0.274)$ \\
\hline Rakke & $0.740(0.010)$ & $0.006(0.165)$ & $0.104(0.075)$ & $43.282(0.203)$ \\
\hline Spock & $0.839(0.010)$ & $0.010(0.235)$ & $0.122(0.123)$ & $43.367(0.387)$ \\
\hline Strolge & & & & \\
\hline Thames & & & & \\
\hline Yowie & $0.460(0.010)$ & $0.012(0.195)$ & $0.229(0.200)$ & $42.327(0.354)$ \\
\hline Zwicky & $0.521(0.010)$ & $-0.254(0.133)$ & $0.185(0.118)$ & $42.471(0.196)$ \\
\hline & $0.670(0.010)$ & $0.183(0.184)$ & $0.164(0.131)$ & $43.123(0.253)$ \\
\hline aphrodite & $1.300(0.010)$ & $-0.096(0.171)$ & $0.211(0.135)$ & $44.858(0.171)$ \\
\hline bilbo & $0.216(0.010)$ & $-0.193(0.172)$ & $0.884(0.171)$ & $40.539(0.229)$ \\
\hline elvis & $0.840(0.010)$ & $0.082(0.175)$ & $0.094(0.083)$ & $43.592(0.206)$ \\
\hline frodo & $0.735(0.010)$ & $0.203(0.176)$ & $0.160(0.125)$ & $43.013(0.227)$ \\
\hline gilgamesh & $1.551(0.010)$ & $-0.033(0.270)$ & $0.483(0.202)$ & $45.153(0.213)$ \\
\hline
\end{tabular}


Table 4-Continued

\begin{tabular}{|c|c|c|c|c|}
\hline SN Ia & $z_{C M B}$ & $\Delta$ & $A_{V}$ & $\mu^{1}$ \\
\hline inanna & $1.307(0.010)$ & $0.165(0.246)$ & $0.234(0.182)$ & $45.182(0.294)$ \\
\hline isis & $0.526(0.010)$ & $-0.333(0.102)$ & $0.294(0.153)$ & $43.383(0.230)$ \\
\hline mcenroe & $0.900(0.010)$ & $-0.267(0.099)$ & $0.234(0.190)$ & $43.425(0.307)$ \\
\hline nanna & $1.140(0.010)$ & $0.249(0.287)$ & $0.084(0.076)$ & $44.456(0.386)$ \\
\hline qiqirn & $0.640(0.010)$ & $0.033(0.199)$ & $0.161(0.133)$ & $42.889(0.292)$ \\
\hline ramone & $0.954(0.010)$ & $0.079(0.181)$ & $0.069(0.069)$ & $44.130(0.283)$ \\
\hline sasquatch & $1.390(0.010)$ & $0.141(0.285)$ & $0.243(0.199)$ & $44.931(0.218)$ \\
\hline torngasak & $1.265(0.010)$ & $-0.068(0.232)$ & $0.230(0.150)$ & $44.843(0.251)$ \\
\hline vilas & $0.935(0.010)$ & $-0.131(0.143)$ & $0.209(0.202)$ & $43.708(0.368)$ \\
\hline b010 & $0.590(0.003)$ & $-0.174(0.189)$ & $0.094(0.079)$ & $42.867(0.244)$ \\
\hline b013 & $0.427(0.003)$ & $-0.043(0.163)$ & $0.118(0.085)$ & $41.940(0.212)$ \\
\hline b020 & $0.426(0.003)$ & $0.073(0.295)$ & $0.139(0.140)$ & $41.669(0.359)$ \\
\hline borg & $1.340(0.010)$ & $-0.237(0.175)$ & $0.349(0.177)$ & $44.919(0.238)$ \\
\hline $\mathrm{d} 033$ & $0.530(0.008)$ & $-0.348(0.116)$ & $0.083(0.071)$ & $42.855(0.168)$ \\
\hline d083 & $0.333(0.001)$ & $-0.341(0.076)$ & $0.085(0.070)$ & $40.636(0.145)$ \\
\hline $\mathrm{d} 084$ & $0.516(0.006)$ & $-0.148(0.221)$ & $0.228(0.179)$ & $42.770(0.318)$ \\
\hline d085 & $0.401(0.008)$ & $-0.307(0.097)$ & $0.136(0.098)$ & $41.949(0.176)$ \\
\hline $\mathrm{d} 086$ & $0.204(0.003)$ & $-0.134(0.132)$ & $0.367(0.245)$ & $40.148(0.270)$ \\
\hline $\mathrm{d} 087$ & $0.337(0.004)$ & $-0.182(0.125)$ & $0.116(0.092)$ & $41.261(0.208)$ \\
\hline d089 & $0.424(0.003)$ & $-0.263(0.128)$ & $0.120(0.088)$ & $41.972(0.175)$ \\
\hline d099 & $0.210(0.002)$ & $-0.138(0.117)$ & $0.105(0.099)$ & $40.312(0.221)$ \\
\hline $\mathrm{d} 117$ & $0.301(0.005)$ & $0.283(0.232)$ & $0.164(0.133)$ & $41.300(0.288)$ \\
\hline d149 & $0.344(0.008)$ & $-0.246(0.120)$ & $0.156(0.123)$ & $41.541(0.215)$ \\
\hline $\mathrm{e} 020$ & $0.159(0.006)$ & $-0.082(0.161)$ & $0.344(0.240)$ & $39.792(0.244)$ \\
\hline $\mathrm{e} 029$ & $0.332(0.008)$ & $0.181(0.263)$ & $0.150(0.149)$ & $41.483(0.291)$ \\
\hline e108 & $0.472(0.007)$ & $-0.359(0.109)$ & $0.056(0.053)$ & $42.230(0.147)$ \\
\hline e132 & $0.235(0.006)$ & $-0.070(0.150)$ & $0.503(0.243)$ & $40.469(0.268)$ \\
\hline e136 & $0.348(0.006)$ & $0.275(0.196)$ & $0.166(0.132)$ & $41.633(0.258)$ \\
\hline e138 & $0.611(0.006)$ & $-0.303(0.130)$ & $0.102(0.077)$ & $42.900(0.204)$ \\
\hline e140 & $0.614(0.006)$ & $-0.222(0.161)$ & $0.104(0.092)$ & $42.812(0.217)$ \\
\hline e147 & $0.641(0.008)$ & $-0.234(0.151)$ & $0.058(0.052)$ & $42.953(0.181)$ \\
\hline e148 & $0.429(0.006)$ & $-0.195(0.151)$ & $0.084(0.063)$ & $42.200(0.181)$ \\
\hline e149 & $0.491(0.006)$ & $-0.013(0.183)$ & $0.124(0.108)$ & $42.160(0.244)$ \\
\hline f011 & $0.536(0.006)$ & $-0.093(0.236)$ & $0.131(0.102)$ & $42.554(0.251)$ \\
\hline f041 & $0.558(0.007)$ & $-0.357(0.109)$ & $0.078(0.064)$ & $42.669(0.159)$ \\
\hline f076 & $0.410(0.004)$ & $0.197(0.269)$ & $0.155(0.116)$ & $41.365(0.293)$ \\
\hline $\mathrm{f} 216$ & $0.595(0.011)$ & $-0.102(0.233)$ & $0.116(0.091)$ & $43.191(0.294)$ \\
\hline $\mathrm{f} 221$ & $0.443(0.004)$ & $-0.114(0.202)$ & $0.065(0.066)$ & $42.775(0.238)$ \\
\hline $\mathrm{f} 231$ & $0.620(0.008)$ & $-0.274(0.141)$ & $0.082(0.064)$ & $42.953(0.188)$ \\
\hline $\mathrm{f} 244$ & $0.546(0.005)$ & $-0.057(0.247)$ & $0.111(0.091)$ & $42.614(0.263)$ \\
\hline g001 & $0.268(0.002)$ & $0.192(0.162)$ & $0.080(0.060)$ & $40.699(0.195)$ \\
\hline g005 & $0.218(0.007)$ & $-0.298(0.076)$ & $0.261(0.205)$ & $40.450(0.227)$ \\
\hline g055 & $0.302(0.006)$ & $-0.329(0.108)$ & $0.742(0.273)$ & $41.560(0.313)$ \\
\hline g097 & $0.339(0.004)$ & $-0.256(0.137)$ & $0.306(0.204)$ & $41.413(0.275)$ \\
\hline g160 & $0.507(0.019)$ & $-0.372(0.089)$ & $0.201(0.158)$ & $42.310(0.256)$ \\
\hline g240 & $0.689(0.005)$ & $-0.205(0.167)$ & $0.056(0.049)$ & $42.971(0.219)$ \\
\hline
\end{tabular}


Table 4-Continued

\begin{tabular}{|c|c|c|c|c|}
\hline SN Ia & $z_{C M B}$ & $\Delta$ & $A_{V}$ & $\mu^{1}$ \\
\hline g276 & $0.244(0.001)$ & $-0.064(0.113)$ & $0.342(0.218)$ & $40.550(0.264)$ \\
\hline h283 & $0.495(0.007)$ & $0.147(0.310)$ & $0.210(0.161)$ & $42.352(0.331)$ \\
\hline h319 & $0.478(0.002)$ & $-0.279(0.134)$ & $0.143(0.099)$ & $42.309(0.203)$ \\
\hline h323 & $0.603(0.007)$ & $-0.131(0.187)$ & $0.097(0.090)$ & $42.916(0.248)$ \\
\hline h359 & $0.348(0.005)$ & $-0.231(0.119)$ & $0.206(0.171)$ & $41.895(0.245)$ \\
\hline h363 & $0.211(0.005)$ & $0.069(0.156)$ & $0.470(0.267)$ & $40.478(0.288)$ \\
\hline h364 & $0.344(0.004)$ & $-0.061(0.149)$ & $0.083(0.071)$ & $41.217(0.203)$ \\
\hline $\mathrm{k} 411$ & $0.565(0.003)$ & $-0.165(0.181)$ & $0.113(0.095)$ & $42.362(0.237)$ \\
\hline $\mathrm{k} 425$ & $0.274(0.003)$ & $0.173(0.264)$ & $0.206(0.172)$ & $40.825(0.295)$ \\
\hline $\mathrm{k} 429$ & $0.171(0.006)$ & $-0.135(0.122)$ & $0.122(0.116)$ & $39.800(0.179)$ \\
\hline $\mathrm{k} 430$ & $0.576(0.007)$ & $-0.194(0.197)$ & $0.102(0.081)$ & $43.248(0.230)$ \\
\hline $\mathrm{k} 441$ & $0.671(0.007)$ & $-0.203(0.182)$ & $0.080(0.070)$ & $43.147(0.218)$ \\
\hline $\mathrm{k} 448$ & $0.405(0.007)$ & $0.024(0.324)$ & $0.239(0.200)$ & $42.261(0.362)$ \\
\hline $\mathrm{m} 026$ & $0.656(0.006)$ & $-0.165(0.193)$ & $0.117(0.084)$ & $42.880(0.263)$ \\
\hline $\mathrm{m} 027$ & $0.285(0.003)$ & $-0.141(0.165)$ & $0.325(0.212)$ & $41.441(0.280)$ \\
\hline $\mathrm{m} 032$ & $0.155(0.004)$ & $-0.158(0.105)$ & $0.096(0.101)$ & $39.800(0.205)$ \\
\hline m034 & $0.562(0.006)$ & $-0.083(0.223)$ & $0.122(0.104)$ & $42.667(0.272)$ \\
\hline m039 & $0.249(0.003)$ & $-0.245(0.144)$ & $0.337(0.267)$ & $40.830(0.329)$ \\
\hline m062 & $0.316(0.004)$ & $0.096(0.320)$ & $0.125(0.098)$ & $41.175(0.310)$ \\
\hline m158 & $0.461(0.007)$ & $-0.355(0.114)$ & $0.258(0.164)$ & $42.423(0.261)$ \\
\hline m193 & $0.336(0.006)$ & $-0.174(0.163)$ & $0.096(0.082)$ & $41.199(0.254)$ \\
\hline $\mathrm{n} 256$ & $0.631(0.011)$ & $-0.364(0.095)$ & $0.065(0.057)$ & $43.011(0.153)$ \\
\hline n263 & $0.368(0.007)$ & $0.031(0.144)$ & $0.068(0.059)$ & $41.416(0.183)$ \\
\hline n278 & $0.308(0.006)$ & $-0.012(0.215)$ & $0.119(0.109)$ & $41.089(0.230)$ \\
\hline $\mathrm{n} 285$ & $0.531(0.009)$ & $-0.136(0.215)$ & $0.131(0.120)$ & $42.551(0.282)$ \\
\hline n326 & $0.267(0.006)$ & $0.807(0.264)$ & $0.106(0.112)$ & $40.547(0.319)$ \\
\hline n404 & $0.285(0.006)$ & $-0.005(0.225)$ & $0.442(0.275)$ & $40.642(0.286)$ \\
\hline $\mathrm{p} 425$ & $0.456(0.004)$ & $-0.178(0.225)$ & $0.404(0.230)$ & $42.428(0.320)$ \\
\hline $\mathrm{p} 455$ & $0.285(0.006)$ & $0.052(0.343)$ & $0.165(0.146)$ & $40.916(0.335)$ \\
\hline p524 & $0.511(0.003)$ & $-0.244(0.153)$ & $0.125(0.097)$ & $42.355(0.216)$ \\
\hline p528 & $0.780(0.009)$ & $-0.331(0.113)$ & $0.043(0.039)$ & $43.727(0.166)$ \\
\hline sn90O & $0.031(0.001)$ & $-0.229(0.073)$ & $0.051(0.042)$ & $35.791(0.162)$ \\
\hline sn90af & $0.050(0.001)$ & $0.507(0.119)$ & $0.073(0.064)$ & $36.600(0.187)$ \\
\hline sn91ag & $0.014(0.001)$ & $-0.238(0.067)$ & $0.047(0.040)$ & $34.004(0.239)$ \\
\hline $\operatorname{sn} 92 \mathrm{P}$ & $0.026(0.001)$ & $-0.176(0.079)$ & $0.100(0.061)$ & $35.529(0.175)$ \\
\hline sn92ae & $0.075(0.001)$ & $0.018(0.124)$ & $0.098(0.084)$ & $37.714(0.188)$ \\
\hline sn92ag & $0.026(0.001)$ & $0.050(0.086)$ & $0.312(0.081)$ & $35.212(0.181)$ \\
\hline sn92al & $0.014(0.001)$ & $-0.064(0.061)$ & $0.033(0.027)$ & $33.964(0.228)$ \\
\hline sn92aq & $0.101(0.001)$ & $0.064(0.127)$ & $0.034(0.032)$ & $38.656(0.171)$ \\
\hline sn92bc & $0.020(0.001)$ & $-0.253(0.044)$ & $0.012(0.012)$ & $34.795(0.175)$ \\
\hline sn92bg & $0.036(0.001)$ & $-0.079(0.083)$ & $0.072(0.052)$ & $36.129(0.174)$ \\
\hline sn92bh & $0.045(0.001)$ & $-0.170(0.086)$ & $0.183(0.079)$ & $36.898(0.156)$ \\
\hline sn92bk & $0.058(0.001)$ & $0.320(0.188)$ & $0.043(0.038)$ & $37.160(0.229)$ \\
\hline sn92bl & $0.043(0.001)$ & $0.235(0.120)$ & $0.035(0.029)$ & $36.461(0.176)$ \\
\hline sn92bo & $0.018(0.001)$ & $0.580(0.079)$ & $0.034(0.029)$ & $34.670(0.202)$ \\
\hline sn92bp & $0.079(0.001)$ & $0.009(0.099)$ & $0.036(0.031)$ & $37.754(0.146)$ \\
\hline
\end{tabular}


Table 4-Continued

\begin{tabular}{|c|c|c|c|c|}
\hline SN Ia & $z_{C M B}$ & $\Delta$ & $A_{V}$ & $\mu^{1}$ \\
\hline sn92br & $0.088(0.001)$ & $1.105(0.161)$ & $0.043(0.045)$ & $37.535(0.224)$ \\
\hline sn92bs & $0.063(0.001)$ & $-0.139(0.097)$ & $0.112(0.083)$ & $37.630(0.179)$ \\
\hline sn93B & $0.071(0.001)$ & $-0.138(0.090)$ & $0.133(0.073)$ & $37.736(0.169)$ \\
\hline sn93H & $0.025(0.001)$ & $0.874(0.096)$ & $0.029(0.026)$ & $35.078(0.174)$ \\
\hline sn93O & $0.052(0.001)$ & $-0.030(0.072)$ & $0.048(0.034)$ & $37.078(0.138)$ \\
\hline sn93ac & $0.049(0.001)$ & $-0.058(0.129)$ & $0.204(0.112)$ & $36.892(0.192)$ \\
\hline sn93ag & $0.050(0.001)$ & $-0.019(0.094)$ & $0.102(0.066)$ & $37.103(0.160)$ \\
\hline sn94M & $0.024(0.001)$ & $0.254(0.093)$ & $0.080(0.055)$ & $35.227(0.177)$ \\
\hline sn94S & $0.016(0.001)$ & $-0.173(0.078)$ & $0.047(0.034)$ & $34.312(0.211)$ \\
\hline $\operatorname{sn} 94 \mathrm{~T}$ & $0.036(0.001)$ & $0.746(0.106)$ & $0.053(0.042)$ & $35.859(0.164)$ \\
\hline sn95E & $0.012(0.001)$ & $-0.093(0.066)$ & $1.460(0.064)$ & $33.888(0.265)$ \\
\hline sn95ac & $0.049(0.001)$ & $-0.316(0.052)$ & $0.106(0.055)$ & $36.602(0.129)$ \\
\hline sn95ak & $0.022(0.001)$ & $0.130(0.080)$ & $0.259(0.072)$ & $34.895(0.185)$ \\
\hline sn95bd & $0.014(0.001)$ & $-0.327(0.049)$ & $0.462(0.159)$ & $34.062(0.241)$ \\
\hline sn96C & $0.028(0.001)$ & $-0.177(0.063)$ & $0.132(0.061)$ & $35.906(0.162)$ \\
\hline sn96ab & $0.124(0.001)$ & $0.089(0.159)$ & $0.071(0.070)$ & $38.852(0.213)$ \\
\hline sn96bl & $0.035(0.001)$ & $-0.170(0.077)$ & $0.142(0.068)$ & $36.075(0.152)$ \\
\hline sn96bo & $0.016(0.001)$ & $-0.035(0.078)$ & $0.626(0.071)$ & $34.304(0.213)$ \\
\hline sn96bv & $0.017(0.001)$ & $-0.285(0.072)$ & $0.389(0.093)$ & $34.312(0.220)$ \\
\hline sn97E & $0.013(0.001)$ & $0.312(0.086)$ & $0.085(0.051)$ & $34.102(0.241)$ \\
\hline $\operatorname{sn} 97 \mathrm{Y}$ & $0.017(0.001)$ & $0.007(0.081)$ & $0.096(0.050)$ & $34.550(0.210)$ \\
\hline sn97cn & $0.017(0.001)$ & $1.359(0.057)$ & $0.025(0.020)$ & $34.425(0.194)$ \\
\hline sn97dg & $0.030(0.001)$ & $-0.018(0.084)$ & $0.092(0.052)$ & $36.121(0.157)$ \\
\hline sn97do & $0.011(0.001)$ & $-0.191(0.081)$ & $0.262(0.061)$ & $33.580(0.289)$ \\
\hline sn98V & $0.017(0.001)$ & $-0.089(0.069)$ & $0.145(0.071)$ & $34.353(0.203)$ \\
\hline sn98ab & $0.028(0.001)$ & $-0.180(0.066)$ & $0.268(0.047)$ & $35.267(0.158)$ \\
\hline sn98bp & $0.010(0.001)$ & $1.254(0.047)$ & $0.025(0.020)$ & $33.175(0.287)$ \\
\hline sn98co & $0.017(0.001)$ & $0.398(0.164)$ & $0.123(0.087)$ & $34.475(0.219)$ \\
\hline sn98de & $0.016(0.001)$ & $1.517(0.042)$ & $0.142(0.061)$ & $34.464(0.206)$ \\
\hline $\operatorname{sn} 98 d x$ & $0.054(0.001)$ & $0.105(0.094)$ & $0.029(0.028)$ & $36.903(0.139)$ \\
\hline sn98ef & $0.017(0.001)$ & $0.134(0.094)$ & $0.068(0.050)$ & $34.094(0.213)$ \\
\hline sn98eg & $0.024(0.001)$ & $-0.022(0.116)$ & $0.131(0.074)$ & $35.334(0.177)$ \\
\hline sn99aa & $0.015(0.001)$ & $-0.346(0.032)$ & $0.025(0.021)$ & $34.425(0.206)$ \\
\hline sn99cc & $0.032(0.001)$ & $0.288(0.094)$ & $0.064(0.049)$ & $35.831(0.154)$ \\
\hline sn99cp & $0.010(0.001)$ & $-0.137(0.111)$ & $0.057(0.045)$ & $33.441(0.295)$ \\
\hline sn99da & $0.013(0.001)$ & $1.487(0.043)$ & $0.066(0.049)$ & $33.925(0.245)$ \\
\hline sn99dk & $0.014(0.001)$ & $-0.306(0.053)$ & $0.252(0.058)$ & $34.160(0.229)$ \\
\hline sn99dq & $0.013(0.001)$ & $-0.359(0.034)$ & $0.299(0.051)$ & $33.705(0.229)$ \\
\hline sn99ee & $0.011(0.001)$ & $-0.278(0.037)$ & $0.643(0.041)$ & $33.571(0.277)$ \\
\hline sn99ef & $0.038(0.001)$ & $-0.136(0.085)$ & $0.052(0.039)$ & $36.597(0.160)$ \\
\hline sn99ej & $0.013(0.001)$ & $0.312(0.099)$ & $0.037(0.031)$ & $34.413(0.255)$ \\
\hline sn99ek & $0.018(0.001)$ & $0.056(0.078)$ & $0.312(0.156)$ & $34.379(0.218)$ \\
\hline sn99gd & $0.019(0.001)$ & $-0.009(0.083)$ & $0.842(0.066)$ & $34.969(0.196)$ \\
\hline sn00bh & $0.024(0.001)$ & $-0.080(0.085)$ & $0.086(0.060)$ & $35.245(0.197)$ \\
\hline sn00ca & $0.025(0.001)$ & $-0.166(0.053)$ & $0.017(0.015)$ & $35.181(0.159)$ \\
\hline sn00ce & $0.017(0.001)$ & $-0.251(0.074)$ & $1.177(0.078)$ & $34.895(0.213)$ \\
\hline
\end{tabular}


Table 4-Continued

\begin{tabular}{|c|c|c|c|c|}
\hline SN Ia & $z_{C M B}$ & $\Delta$ & $A_{V}$ & $\mu^{1}$ \\
\hline sn00cf & $0.036(0.001)$ & $-0.005(0.079)$ & $0.086(0.055)$ & $36.316(0.145)$ \\
\hline sn00cn & $0.023(0.001)$ & $0.724(0.084)$ & $0.071(0.060)$ & $35.056(0.170)$ \\
\hline sn00dk & $0.016(0.001)$ & & $0.017(0.0$ & 34.333 \\
\hline sn00fa & $0.022(0.001)$ & $-0.138(0.077)$ & $0.287(0.056)$ & $34.986(0.185)$ \\
\hline sn01G & $0.017(0.001)$ & $-0.195(0.073)$ & $0.050(0.035)$ & $34.481(0.202)$ \\
\hline sn01ah & $0.058(0.001)$ & $-0.248(0.109)$ & $0.042(0.033)$ & $37.185(0.167)$ \\
\hline sn01az & $0.041(0.001)$ & $-0.008(0.1$ & $0.049(0$ & 36 . \\
\hline sn01ba & $0.030(0.001)$ & $-0.164(0.058)$ & $0.025(0.021)$ & $35.821(0.147)$ \\
\hline sn01bf & $0.015(0.001)$ & $-0.242(0$. & $0.170(0.068)$ & 34.059 \\
\hline sn01bt & $0.014(0.001)$ & $0.041(0.069)$ & $0.426(0.063)$ & $34.025(0.228)$ \\
\hline sn01cn & $0.015(0.001)$ & $-0.016(0.0$ & $0.309(0.0$ & $34.126(0.218)$ \\
\hline sn01cp & $0.022(0.001)$ & $-0.033(0.1$ & $0.054(0.0$ & 34.998 \\
\hline $\mathrm{sn} 01 \mathrm{cz}$ & $0.016(0$ & $-0.124(0.0$ & $0.200(C$ & $34.260(0.209)$ \\
\hline sn01da & $0.016(C$ & $-0.065(0.0$ & $0.075(0$. & $34.554(0.222)$ \\
\hline sn01eh & $0.036(0.001)$ & $-0.371(0.043)$ & $0.036(0.029)$ & $36.266(0.130)$ \\
\hline sn01en & $0.015(0.001)$ & $0.043(0.0$ & 0.085 & 34.237 \\
\hline sn01ep & 0.013 & 0.046( & 0.255 & 33.89 \\
\hline sn01fe & 0.014 & -0.169( & 0.09 & 34.10 \\
\hline sn01fh & $0.011(0.001)$ & $0.632(0.124)$ & $0.077(($ & .277) \\
\hline sn01gb & $0.027(0.0$ & $-0.261(0.128)$ & $1.232(0.1$ & 35.544 \\
\hline sn01ic & $0.043(0.001)$ & $0.292(0.212)$ & $0.180(0.125)$ & 36.438 \\
\hline sn01ie & 0.03 & $-0.020(0.1$ & 0.150 & 35.781 \\
\hline $\operatorname{sn} 01 n$ & $0.022(0.001)$ & $-0.054(0.1$ & $0.487(0.1$ & 35.102 \\
\hline sn01v & $0.016(0.001)$ & $-0.330(0.043)$ & $0.171(0.041)$ & $34.047(0.202)$ \\
\hline sn02bf & $0.025(0.001)$ & $-0.242(0.082)$ & $0.268(0.086)$ & $35.458(0.180)$ \\
\hline sn02bz & 0.03 & & 0.0 & 36 . \\
\hline sn02ck & 0.030 & $-0.117(0.088)$ & 0.05 & 35. \\
\hline $\mathrm{sn} 02 \mathrm{cr}$ & $0.010(0.001)$ & $0.009(0.083)$ & $0.122(0.063)$ & $33.458(0.290)$ \\
\hline sn02de & $0.028(0.001)$ & $-0.224(0.1$ & $0.382(0.084)$ & $35.706(0.161)$ \\
\hline sn02 & 0.01 & & & \\
\hline sn02 & 0.015( & 0.76 & 0.03 & 34. \\
\hline sn02dp & $0.011(0.001)$ & $0.023(0.135)$ & $0.268(0.090)$ & $33.565(0.287)$ \\
\hline sn02fb & $0.015(0.001)$ & $1.368(0.089)$ & $0.238(0.084)$ & $34.368(0.220)$ \\
\hline $\mathrm{sn} 02 \mathrm{~g}$ & 0.03 & $0.565(0.3$ & $0.409(0.1$ & $35.676(0.349)$ \\
\hline sn02ha & $0.013(0.001)$ & $0.127(0.0$ & $0.042(0.032)$ & $34.013(0.238)$ \\
\hline sn02hd & $0.036(0.001)$ & $0.137(0.132)$ & $0.145(0.081)$ & $35.797(0.167)$ \\
\hline sn02he & $0.025(0.001)$ & $0.423(0.102)$ & $0.031(0.026)$ & $35.249(0.192)$ \\
\hline sn02hu & $0.038(0.001)$ & $-0.246(0.055)$ & $0.036(0.030)$ & $36.234(0.132)$ \\
\hline sn02hw & 0.016 & $0.704(0.1$ & 0.60 & $34.330(0.212)$ \\
\hline sn02jy & $0.020(0.001)$ & $-0.221(0.078)$ & $0.103(0.056)$ & $35.188(0.181)$ \\
\hline sn02kf & $0.019(0.001)$ & $0.135(0.070)$ & $0.030(0.025)$ & $34.977(0.188)$ \\
\hline & $0.024(0.001)$ & $1.121(0.138)$ & $0.215(0.128)$ & $34.984(0.186)$ \\
\hline $\mathrm{sn} 03 \mathrm{U}$ & $0.028(0.001)$ & $0.428(0.080)$ & $0.044(0.033)$ & $35.462(0.164)$ \\
\hline sn03W & $0.021(0.001)$ & $-0.071(0.059)$ & $0.330(0.050)$ & $34.867(0.175)$ \\
\hline $\mathrm{sn} 03 \mathrm{ch}$ & $0.030(0.001)$ & $0.063(0.101)$ & $0.039(0.031)$ & $35.984(0.158)$ \\
\hline sn03cq & $0.034(0.001)$ & $0.012(0.114)$ & $0.411(0.144)$ & $36.017(0.201)$ \\
\hline
\end{tabular}


Table 4-Continued

\begin{tabular}{|c|c|c|c|c|}
\hline SN Ia & $B$ & $\Delta$ & $A_{V}$ & $\mu^{1}$ \\
\hline sn03fa & $0.039(0.001)$ & $-0.356(0.045)$ & $0.030(0.022)$ & $36.353(0.128)$ \\
\hline sn03hu & $0.075(0.001)$ & $-0.311(0.097)$ & $0.225(0.117)$ & $37.642(0.165)$ \\
\hline sn03ic & $0.054(0.001)$ & $0.171(0.134)$ & $0.046(0.045)$ & $36.776(0.158)$ \\
\hline sn03it & $0.024(0.001)$ & $0.374(0.109)$ & $0.083(0.055)$ & $35.282(0.187)$ \\
\hline sn03iv & $0.034(0.001)$ & $0.278(0.106)$ & $0.023(0.024)$ & $36.258(0.160)$ \\
\hline sn04as & $0.032(0.001)$ & $-0.184(0.070)$ & $0.303(0.058)$ & $36.145(0.150)$ \\
\hline sn04bg & $0.022(0.001)$ & $-0.138(0.070)$ & $0.067(0.052)$ & $35.096(0.181)$ \\
\hline sn04l & $0.033(0.001)$ & $0.029(0.125)$ & $0.419(0.092)$ & $36.056(0.172)$ \\
\hline sn05dv & $0.011(0.001)$ & $1.190(0.237)$ & $0.679(0.230)$ & $33.133(0.291)$ \\
\hline sn05el & $0.015(0.001)$ & $0.210(0.060)$ & $0.012(0.013)$ & $34.242(0.220)$ \\
\hline sn05eq & $0.028(0.001)$ & $-0.309(0.045)$ & $0.104(0.047)$ & $35.797(0.148)$ \\
\hline sn05eu & $0.034(0.001)$ & $-0.319(0.056)$ & $0.052(0.038)$ & $36.118(0.147)$ \\
\hline sn05hc & $0.045(0.001)$ & $-0.125(0.075)$ & $0.115(0.052)$ & $36.755(0.136)$ \\
\hline sn05hf & $0.042(0.001)$ & $0.070(0.108)$ & $0.036(0.034)$ & $36.590(0.162)$ \\
\hline sn05hj & $0.057(0.001)$ & $-0.325(0.088)$ & $0.112(0.067)$ & $37.261(0.140)$ \\
\hline sn05iq & $0.033(0.001)$ & $0.137(0.073)$ & $0.031(0.026)$ & $36.129(0.148)$ \\
\hline sn05ir & $0.075(0.001)$ & $-0.395(0.091)$ & $0.093(0.058)$ & $38.036(0.135)$ \\
\hline sn05kc & $0.014(0.001)$ & $0.036(0.082)$ & $0.624(0.074)$ & $34.084(0.235)$ \\
\hline sn05ki & $0.021(0.001)$ & $0.285(0.066)$ & $0.018(0.015)$ & $34.803(0.181)$ \\
\hline sn05ls & $0.021(0.001)$ & $-0.254(0.062)$ & $0.750(0.064)$ & $34.694(0.186)$ \\
\hline sn05lz & $0.040(0.001)$ & $0.217(0.103)$ & $0.173(0.068)$ & $36.557(0.155)$ \\
\hline $\mathrm{sn} 05 \mathrm{mc}$ & $0.026(0.001)$ & $0.935(0.071)$ & $0.077(0.051)$ & $35.429(0.159)$ \\
\hline $\mathrm{sn} 05 \mathrm{~ms}$ & $0.026(0.001)$ & $-0.159(0.052)$ & $0.070(0.040)$ & $35.618(0$. \\
\hline $\mathrm{sn} 05 \mathrm{mz}$ & $0.017(0.001)$ & $1.364(0.067)$ & $0.266(0.089)$ & $34.297(0.203)$ \\
\hline sn05na & $0.027(0.001)$ & $0.021(0.065)$ & $0.052(0.037)$ & $35.359(0.161)$ \\
\hline sn06ac & $0.024(0.001)$ & $0.161(0.079)$ & $0.104(0.047)$ & $35.255(0.172)$ \\
\hline sn06ak & $0.039(0.001)$ & $0.191(0.122)$ & $0.044(0.038)$ & $36.282(0.186)$ \\
\hline sn06al & $0.069(0.001)$ & $0.109(0.106)$ & $0.034(0.029)$ & $37.798(0.142)$ \\
\hline sn06an & $0.065(0.001)$ & $-0.238(0.090)$ & $0.077(0.051)$ & $37.654(0.139)$ \\
\hline sn06ar & $0.023(0.001)$ & $0.428(0.243)$ & $0.196(0.124)$ & $35.151(0.223)$ \\
\hline $\operatorname{sn} 06 a x$ & $0.018(0.001)$ & $-0.162(0.048)$ & $0.038(0.029)$ & $34.593(0.188)$ \\
\hline sn06az & $0.032(0.001)$ & $0.154(0.056)$ & $0.012(0.012)$ & $35.803(0.143)$ \\
\hline sn06bd & $0.026(0.001)$ & $1.510(0.090)$ & $0.091(0.069)$ & $35.562(0.170)$ \\
\hline sn06bq & $0.021(0.001)$ & $0.323(0.075)$ & $0.092(0.059)$ & $35.111(0.175)$ \\
\hline sn06bt & $0.033(0.001)$ & $-0.325(0.052)$ & $0.428(0.053)$ & $35.993(0.143)$ \\
\hline sn06bu & $0.084(0.001)$ & $-0.333(0.107)$ & $0.047(0.040)$ & $37.958(0.137)$ \\
\hline sn06bw & $0.031(0.001)$ & $0.514(0.137)$ & $0.133(0.113)$ & $35.663(0.178)$ \\
\hline sn06bz & $0.028(0.001)$ & $1.502(0.082)$ & $0.182(0.115)$ & $35.380(0.161)$ \\
\hline sn06cc & $0.033(0.001)$ & $-0.226(0.058)$ & $0.812(0.051)$ & $36.194(0.143)$ \\
\hline sn06cf & $0.042(0.001)$ & $-0.080(0.108)$ & $0.055(0.049)$ & $36.478(0.148)$ \\
\hline sn06cg & $0.029(0.001)$ & $0.651(0.170)$ & $0.142(0.098)$ & $35.109(0.185)$ \\
\hline sn06cj & $0.068(0.001)$ & $-0.228(0.106)$ & $0.036(0.027)$ & $37.776(0.129)$ \\
\hline sn06cp & $0.023(0.001)$ & $-0.172(0.087)$ & $0.440(0.064)$ & $35.005(0.178)$ \\
\hline sn06cq & $0.049(0.001)$ & $-0.263(0.113)$ & $0.136(0.073)$ & $37.011(0.160)$ \\
\hline $\mathrm{sn} 06 \mathrm{cs}$ & $0.024(0.001)$ & $1.525(0.104)$ & $0.245(0.190)$ & $35.237(0.183)$ \\
\hline sn06ef & $0.017(0.001)$ & $0.077(0.094)$ & $0.055(0.043)$ & $34.770(0.228)$ \\
\hline
\end{tabular}


Table 4-Continued

\begin{tabular}{|c|c|c|c|c|}
\hline SN Ia & $B$ & $\Delta$ & $A_{V}$ & $\mu^{1}$ \\
\hline sn06ej & $0.019(0.001)$ & $0.181(0.087)$ & $0.037(0.031)$ & $34.858(0.202)$ \\
\hline sn06en & $0.031(0.001)$ & $-0.053(0.081)$ & 0.177 & 35.93 \\
\hline sn06et & $0.021(0.001)$ & $-0.233(0.078)$ & $0.328(0.074)$ & $35.065(0.192)$ \\
\hline sn06gj & $0.028(0.001)$ & $0.582(0$ & 6) & 35.672( \\
\hline sn06gr & $0.034(0.001)$ & $-0.305(0.046)$ & $0.304(0.052)$ & $36.206(0.143)$ \\
\hline sn06gt & $0.044(0.001)$ & $0.689(0.147)$ & $0.067(0.068)$ & $36.727(0.173)$ \\
\hline sn06hc & $0.013(0.001)$ & $1.297(0.072)$ & $0.287(0.125)$ & $34.258(0.236)$ \\
\hline sn06hb & $0.015(0.001)$ & $0.686(0.099)$ & $0.055(0.041)$ & $34.044(0.218)$ \\
\hline sn06ke & $0.017(0.001)$ & $1.479(0.117)$ & $1.006(0.203)$ & $34.983(0.223)$ \\
\hline sn06kf & $0.021(0.001)$ & $0.628(0.0$ & $0.024(0$ & $34.961(0.195)$ \\
\hline sn06le & $0.017(0.001)$ & $-0.272(0.044)$ & & \\
\hline sn06lf & $0.013(0.001)$ & $0.292(0.079)$ & & \\
\hline sn06mo & $0.036(0.001)$ & $0.614(($ & 0.039 & 36.1 \\
\hline $\mathrm{sn} 06 \mathrm{mp}$ & $0.023(0.001)$ & $-0.121(0$ & $0.166(C$ & 35.258 \\
\hline sn06n & & & & \\
\hline sn06nz & 0037 (0 & $1.115(0$ & & \\
\hline sn06oa & 0.059 (C & -0.25 & $0.1 \xi$ & 37.26 \\
\hline sn06ob & & 0.541 & $0.021(0$ & 37.10 \\
\hline sn06on & & -0.1 & & \\
\hline sn06or & 0.02 & & & \\
\hline sn06os & 0.03 & -0.125( & 0.858 & 35.773 \\
\hline sn06qo & 0.03 & $-0.182(0.0$ & $0.453(0.0$ & 35. \\
\hline $\mathrm{sn} 06 \mathrm{~s}$ & & -0 . & & 36. \\
\hline $\mathrm{sn} 06 \mathrm{sr}$ & 0.0 & & 0.0 & 35.2 \\
\hline sn06td & $0.015(0.001)$ & 0.390( & $0.171(0$. & $34.464(0.244)$ \\
\hline sn06te & $0.032(0.001)$ & $-0.073(0$. & $0.037(0.031)$ & $35.992(0.155)$ \\
\hline sn07ae & & & $0.085(0.0$ & $37.270(0.148)$ \\
\hline sn07ai & 0.0 & -0.3 & 0.33 & 35.990 \\
\hline sn07al & 0.013 & $1.363(0$ & $0.229(0$ & $34.337(0.245)$ \\
\hline sn07ap & $0.016(0.001)$ & $0.353(0.093)$ & $0.013(0.013)$ & $34.495(0.227)$ \\
\hline sn07ar & & $1.296(0.1$ & & $36.477(0.183)$ \\
\hline sn07au & 0.02 & 1.0 & 0.0 & 34. \\
\hline sn07ba & 0.039 & $1.209(0.097)$ & $0.082(0.0$ & $35.803(0.146)$ \\
\hline sn07bc & $0.022(0.001)$ & $0.285(0.090)$ & $0.084(0.059)$ & $34.931(0.187)$ \\
\hline & & & & \\
\hline sn07bz & 0.02 & -0.06 & $0.242(\mathrm{C}$ & $35.761(0.185)$ \\
\hline sn07ca & $0.015(0.001)$ & $-0.214(0.057)$ & $0.580(0.069)$ & $34.621(0.222)$ \\
\hline sn07cg & $0.034(0.001)$ & $-0.032(0.184)$ & $1.141(0.158)$ & $35.718(0.203)$ \\
\hline $\mathrm{sn} 07 \mathrm{ci}$ & $0.019(0.001)$ & $0.883(0.078)$ & $0.074(0.063)$ & $34.289(0.191)$ \\
\hline sn07co & $0.027(0.001)$ & $-0.041(0.0$ & $0.392(0.0$ & $35.420(0.158)$ \\
\hline $\mathrm{sn} 07 \mathrm{cq}$ & $0.025(0.001)$ & $0.052(0.071)$ & $0.109(0.059)$ & $35.084(0.174)$ \\
\hline $\mathrm{sn} 07 \mathrm{f}$ & $0.024(0.001)$ & $-0.140(0.051)$ & $0.047(0.038)$ & $35.355(0.162)$ \\
\hline $\operatorname{sn} 07 \mathrm{n}$ & $0.014(0.001)$ & $1.403(0.103)$ & $0.544(0.133)$ & $34.419(0.241)$ \\
\hline $\mathrm{sn} 07 \mathrm{o}$ & $0.036(0.001)$ & $-0.005(0.080)$ & $0.052(0.039)$ & $36.115(0.155)$ \\
\hline sn07qe & $0.023(0.001)$ & $-0.257(0.049)$ & $0.148(0.059)$ & $35.453(0.164)$ \\
\hline $\mathrm{sn} 07 \mathrm{r}$ & $0.031(0.001)$ & $0.189(0.086)$ & $0.027(0.026)$ & $35.877(0.162)$ \\
\hline
\end{tabular}


Table 4-Continued

\begin{tabular}{lcrcc}
\hline \hline SN Ia & $z_{C M B}$ & $\Delta$ & $A_{V}$ & $\mu^{1}$ \\
\hline sn07s & $0.015(0.001)$ & $-0.323(0.040)$ & $0.833(0.054)$ & $34.221(0.230)$ \\
sn08L & $0.019(0.001)$ & $0.293(0.180)$ & $0.036(0.033)$ & $34.392(0.258)$ \\
sn08af & $0.034(0.001)$ & $0.245(0.104)$ & $0.056(0.045)$ & $35.986(0.171)$ \\
sn08bf & $0.026(0.001)$ & $-0.179(0.064)$ & $0.102(0.049)$ & $35.173(0.159)$ \\
\hline
\end{tabular}

${ }^{1}$ Distance moduli were calculated with $\mathrm{H}_{\mathrm{o}}=65 \mathrm{kms}^{-1}$ and $M_{V}=$ -19.504 . Uncertainty of $\mu$ includes $0.078 \mathrm{mag}$ "intrinsic", $\sigma_{v-p e c}(400$ $\mathrm{km} \mathrm{s}^{-1}$ ), and $\sigma_{z}$. 
Table 5. $\mathrm{SN}+\mathrm{BAO}$ Values of $1+w$ for Each Fitter.

\begin{tabular}{|c|c|c|c|c|}
\hline Fitter & OLD & CfA3 & $\mathrm{OLD}+\mathrm{CfA} 3$ & OLD-CfA3 \\
\hline Best cuts: $\Delta, A_{V}, c$ & $1+w$ & $1+w$ & $1+w$ & $\Delta(1+w)$ \\
\hline SALT & $0.036_{-0.082}^{+0.080}$ & $-0.016_{-0.080}^{+0.078}$ & $0.026_{-0.071}^{+0.069}$ & +0.052 \\
\hline SALT2 & $-0.075_{-0.097}^{+0.092}$ & $0.071_{-0.087}^{+0.083}$ & $0.026_{-0.082}^{+0.078}$ & -0.147 \\
\hline MLCS31 & $0.006_{-0.080}^{+0.077}$ & $0.099_{-0.067}^{+0.066}$ & $0.074_{-0.065}^{+0.063}$ & -0.093 \\
\hline MLCS17 & $0.083_{-0.079}^{+0.076}$ & $0.134_{-0.067}^{+0.065}$ & $0.118_{-0.065}^{+0.063}$ & -0.051 \\
\hline Mean & $0.012_{-0.085}^{+0.081}$ & $0.072_{-0.075}^{+0.073}$ & $0.061_{-0.071}^{+0.068}$ & -0.068 \\
\hline Stddev $1+w$ & 0.066 & 0.064 & 0.044 & \\
\hline Minimal cuts & $1+w$ & $1+w$ & $1+w$ & $\Delta(1+w)$ \\
\hline SALT & $-0.011_{-0.080}^{+0.078}$ & $-0.002_{-0.075}^{+0.073}$ & $0.013_{-0.068}^{+0.066}$ & -0.010 \\
\hline SALT2 & $\begin{array}{r}-0.097_{-0.102}^{+0.098} \\
\end{array}$ & $0.054_{-0.082}^{+0.079}$ & $0.007_{-0.079}^{+0.075}$ & -0.151 \\
\hline MLCS31 & $-0.072_{-0.085}^{+0.082}$ & $0.005_{-0.073}^{+0.071}$ & $-0.018_{-0.071}^{+0.068}$ & -0.077 \\
\hline MLCS17 & $0.073_{-0.082}^{+0.079}$ & $0.108_{-0.069}^{+0.068}$ & $0.099_{-0.067}^{+0.066}$ & -0.036 \\
\hline \multicolumn{5}{|l|}{$A_{V}$ cuts } \\
\hline MLCS31 & $-0.040_{-0.084}^{+0.081}$ & $0.065_{-0.071}^{+0.069}$ & $0.031_{-0.069}^{+0.067}$ & -0.105 \\
\hline MLCS17 & $0.045_{-0.083}^{+0.080}$ & $0.085_{-0.070}^{+0.068}$ & $0.076_{-0.066}^{+0.066}$ & -0.040 \\
\hline \multicolumn{5}{|l|}{$\Delta<0.7$} \\
\hline MLCS31 & $-0.040_{-0.086}^{+0.083}$ & $0.038_{-0.073}^{+0.071}$ & $0.016_{-0.070}^{+0.068}$ & -0.078 \\
\hline MLCS17 & & & $0.131_{-0.067}^{+0.066}$ & \\
\hline \multicolumn{5}{|l|}{$\mathrm{No}-\mathrm{U}$} \\
\hline MLCS31 & $-0.046_{-0.086}^{+0.083}$ & $0.039_{-0.074}^{+0.072}$ & $0.012_{-0.071}^{+0.069}$ & -0.085 \\
\hline MLCS17 & & $0.106_{-0.074}^{+0.072}$ & & \\
\hline SALT2 & $-0.078_{-0.012}^{+0.012}$ & $0.055_{-0.090}^{+0.087}$ & $0.012_{-0.086}^{+0.083}$ & -0.134 \\
\hline \multicolumn{5}{|l|}{$\mathrm{No}-\mathrm{U} ; A_{V}$ cut } \\
\hline MLCS31 & $-0.033_{-0.086}^{+0.083}$ & $0.074_{-0.072}^{+0.071}$ & $0.040_{-0.070}^{+0.068}$ & -0.108 \\
\hline MLCS17 & & $0.097_{-0.076}^{+0.074}$ & & \\
\hline \multicolumn{5}{|l|}{$\mathrm{No}-\mathrm{U} ; A_{V}, \Delta$ cuts } \\
\hline MLCS31 & $0.013_{-0.088}^{+0.086}$ & $0.110_{-0.074}^{+0.073}$ & $0.081_{-0.071}^{+0.069}$ & -0.098 \\
\hline MLCS17 & & $0.148_{-0.075}^{+0.074}$ & & \\
\hline
\end{tabular}

Note. - The last column is the difference in $1+w$ between the OLD and the CfA3 samples. Below the four rows are the mean and standard deviation of the values from the four fitters. The top section shows $1+w$ for the uncut samples (no cuts beyond the original cuts on $t_{1 s t}, \chi_{\nu}^{2}, z$, and $A_{V}$ ) and the second section shows $1+w$ after the "best" cuts $\left(-0.1<c<0.2, A_{V} \leq 0.5\right)$. Below are results from other cuts and fits as described in the text. 
Table 6. Mean of the Minimal-Cuts Hubble Residuals by Sample and Fitter.

\begin{tabular}{|c|c|c|c|c|c|c|}
\hline Sample & $N$ & Mean & StdDev & StdDev $/ \sqrt{N}$ & WMEAN & $\sigma_{W M}$ \\
\hline \multicolumn{7}{|l|}{ MLCS31 } \\
\hline OLD & 72 & -0.0385 & 0.209 & 0.0246 & $\begin{array}{l}-0.0233 \\
\end{array}$ & 0.0221 \\
\hline CfA3 & 129 & -0.0099 & 0.215 & 0.0189 & 0.0150 & 0.0159 \\
\hline $\mathrm{OLD}+\mathrm{CfA} 3$ & 201 & -0.0202 & 0.213 & 0.0150 & 0.0019 & 0.0129 \\
\hline High-z & 165 & -0.0099 & 0.236 & 0.0183 & -0.0009 & 0.0166 \\
\hline OLD-CfA3 & & -0.0286 & & 0.0310 & -0.0383 & 0.0272 \\
\hline \multicolumn{7}{|l|}{ MLCS17 } \\
\hline OLD & 70 & -0.0132 & 0.200 & 0.0239 & -0.0121 & 0.0223 \\
\hline CfA3 & 133 & 0.0028 & 0.207 & 0.0179 & 0.0062 & 0.0153 \\
\hline $\mathrm{OLD}+\mathrm{CfA} 3$ & 203 & -0.0027 & 0.204 & 0.0143 & 0.0004 & 0.0126 \\
\hline High-z & 169 & 0.0058 & 0.224 & 0.0172 & 0.0063 & 0.0167 \\
\hline OLD-CfA3 & & -0.0160 & & 0.0299 & -0.0183 & 0.0270 \\
\hline \multicolumn{7}{|l|}{$\overline{\text { SALT }}$} \\
\hline OLD & 57 & -0.0206 & 0.186 & 0.0246 & -0.0136 & 0.0192 \\
\hline CfA3 & 90 & 0.0091 & 0.164 & 0.0172 & 0.0035 & 0.0185 \\
\hline $\mathrm{OLD}+\mathrm{CfA} 3$ & 147 & -0.0024 & 0.173 & 0.0142 & -0.0047 & 0.0133 \\
\hline High-z & 250 & 0.0150 & 0.394 & 0.0249 & -0.0069 & 0.0119 \\
\hline OLD-CfA3 & & -0.0297 & & 0.0301 & -0.0170 & 0.0267 \\
\hline \multicolumn{7}{|l|}{ SALT2 } \\
\hline OLD & 65 & -0.0306 & 0.204 & 0.0253 & -0.0347 & 0.0258 \\
\hline CfA3 & 115 & 0.0236 & 0.183 & 0.0170 & 0.0210 & 0.0194 \\
\hline $\mathrm{OLD}+\mathrm{CfA} 3$ & 180 & 0.0040 & 0.192 & 0.0143 & 0.0009 & 0.0155 \\
\hline High-z & 171 & 0.0466 & 0.311 & 0.0238 & 0.0040 & 0.0177 \\
\hline OLD-CfA3 & & -0.0542 & & 0.0305 & -0.0558 & 0.0323 \\
\hline
\end{tabular}

Note. - Mean and weighted mean of the Hubble residuals, relative to the best-fit cosmology, for each fitter and sample. $N$ is the number of SN Ia in each sample for each fitter. The standard deviation of the Hubble residuals and the uncertainties of the mean and the weighted mean are shown. The bottom row for each fitter contains the difference in the means and the weighted means of the OLD sample minus the CfA3 sample, and their uncertainties. 
Table 7. Weighted Mean of the Hubble Residuals of Survey Subsamples

\begin{tabular}{|c|c|c|c|c|c|c|c|c|}
\hline Sample & & MLCS17 & & MLCS31 & & SALT & & SALT2 \\
\hline Minimal Cuts & $\mathrm{N}$ & WMEAN & $\mathrm{N}$ & WMEAN & $\mathrm{N}$ & WMEAN & $\mathrm{N}$ & WMEAN \\
\hline CalanTololo & 21 & $-0.022(0.039)$ & 22 & $-0.034(0.038)$ & 17 & $0.043(0.036)$ & 20 & $-0.077(0.044)$ \\
\hline CfA1 & 13 & $-0.064(0.051)$ & 12 & $-0.115(0.056)$ & 11 & $-0.031(0.057)$ & 12 & $-0.068(0.059)$ \\
\hline CfA2 & 25 & $0.028(0.038)$ & 26 & $-0.003(0.038)$ & 16 & $-0.010(0.066)$ & 22 & $0.054(0.046)$ \\
\hline CfA3(4Sh) & 45 & $-0.008(0.028)$ & 43 & $-0.007(0.029)$ & 29 & $-0.038(0.033)$ & 40 & $-0.020(0.035)$ \\
\hline CfA3(Kep) & 88 & $0.013(0.018)$ & 86 & $0.024(0.019)$ & 61 & $0.029(0.022)$ & 75 & $0.038(0.023)$ \\
\hline Other & 11 & $-0.015(0.061)$ & 11 & $0.032(0.056)$ & 5 & $-0.117(0.063)$ & 11 & $-0.083(0.068)$ \\
\hline K08 & & & & & 8 & $-0.015(0.030)$ & & \\
\hline StdDev & & 0.032 & & 0.054 & & 0.052 & & 0.060 \\
\hline StdDev* & & 0.021 & & 0.027 & & 0.033 & & 0.061 \\
\hline OLD-CfA3 & & $-0.018(0.027)$ & & $-0.038(0.027)$ & & $-0.017(0.027)$ & & $-0.056(0.032)$ \\
\hline OLD*_CfA3 & & & & & & $-0.011(0.027)$ & & $-0.036(0.038)$ \\
\hline \multicolumn{9}{|l|}{$A_{V}, \Delta$ Cuts } \\
\hline CalanTololo & 19 & $0.005(0.040)$ & 19 & $-0.017(0.042)$ & & & & \\
\hline CfA1 & 10 & $-0.054(0.057)$ & 7 & $-0.082(0.070)$ & & & & \\
\hline CfA2 & 22 & $0.008(0.041)$ & 23 & $-0.037(0.040)$ & & & & \\
\hline CfA3(4Sh) & 41 & $-0.008(0.029)$ & 33 & $-0.033(0.032)$ & & & & \\
\hline CfA3(Kep) & 73 & $0.010(0.020)$ & 65 & $0.034(0.021)$ & & & & \\
\hline Other & 10 & $-0.027(0.063)$ & 8 & $0.025(0.061)$ & & & & \\
\hline StdDev & & 0.025 & & 0.043 & & & & \\
\hline StdDev* & & 0.015 & & 0.033 & & & & \\
\hline OLD-CfA3 & & $-0.013(0.029)$ & & $-0.039(0.030)$ & & & & \\
\hline $\mathrm{OLD}^{*}-\mathrm{CfA} 3$ & & $-0.003(0.031)$ & & $-0.031(0.031)$ & & & & \\
\hline
\end{tabular}

Note. - The nearby sample is broken up into subsamples. The top half is for the minimal cuts while $0.7 \leq \Delta \leq 1.2$ and $A_{V}>0.5$ are excluded from MLCS2k2 in the bottom half. The weighted mean of the residuals is presented for each subsample. The standard deviation of the weighted means is presented below. The difference between the weighted means of the residuals, OLD-CfA3, is presented. * means the most-discrepant subsample is excluded from OLD. 
Table 8. Hubble Bubble Results.

\begin{tabular}{l|l|llll}
\hline \hline \multirow{2}{*}{ Fitter } & Bubble Sample & $z$ (partition) & $\delta_{H}$ & $\sigma$ & $\delta_{H} / \sigma$ \\
\hline \multirow{2}{*}{ MLCS31 } & Old+CfA3 & 0.028 & 0.029 & 0.005 & 5.556 \\
& Old & 0.025 & 0.036 & 0.012 & 2.933 \\
& CfA3 & 0.040 & 0.033 & 0.009 & 3.660 \\
\hline \multirow{2}{*}{ MLCS17 } & Old+CfA3 & 0.034 & -0.020 & 0.007 & 2.752 \\
& Old & 0.046 & -0.027 & 0.021 & 1.301 \\
& CfA3 & 0.034 & -0.027 & 0.008 & 3.467 \\
\hline SALT & Old+CfA3 & 0.035 & -0.014 & 0.010 & 1.306 \\
& Old & 0.025 & 0.037 & 0.018 & 2.089 \\
& CfA3 & 0.035 & -0.040 & 0.008 & 4.714 \\
\hline SALT2 & Old+CfA3 & 0.045 & -0.019 & 0.016 & 1.201 \\
& Old & 0.018 & 0.015 & 0.052 & 0.292 \\
& CfA3 & 0.034 & -0.024 & 0.015 & 1.635 \\
\hline & $A_{V} \leq 0.5$ & & & & \\
\hline \multirow{2}{*}{ MLCS31 } & Old+CfA3 & 0.029 & 0.015 & 0.011 & 1.289 \\
& Old & 0.025 & 0.019 & 0.026 & 0.714 \\
& CfA3 & 0.029 & 0.015 & 0.017 & 0.891 \\
\hline MLCS17 & Old+CfA3 & 0.015 & -0.028 & 0.018 & 1.542 \\
& Old & 0.026 & 0.027 & 0.017 & 1.539 \\
& CfA3 & 0.034 & -0.021 & 0.011 & 1.960 \\
\hline
\end{tabular}

Note. - The Hubble bubble calculations are listed for the four fitters and three SN Ia samples. The partition redshift, void amplitude, $\delta_{H}$, void amplitude uncertainty, $\sigma$, and void significance, $\delta_{H} / \sigma$, are presented. The MLCS2k2 Hubble bubble results with $A_{V} \leq 0.5$ are at the bottom. The most robust results are from the OLD+CfA3 sample for SALT and SALT2 and for MLCS31 and MLCS17 with $A_{V} \leq 0.5$, all with absolutevalue void significances just greater than one. The low significance and the different partition redshifts of the most significant voids weakens the case for a Hubble bubble. 
Table 9. Std. Dev. and Weighted Means of Hubble Residuals by Host-Galaxy Morpology.

\begin{tabular}{l|l|r|rr|rr}
\hline \hline \multicolumn{1}{c|}{ Fitter } & \multicolumn{1}{c}{ Morph } & $\mathrm{N}$ & StdDev & WMEAN & RelativeWM & $\sigma_{W M}$ \\
\hline \multirow{2}{*}{ SALT } & E-S0 & 21 & 0.170 & -0.0641 & -0.0712 & 0.0366 \\
& S0a-Sc & 63 & 0.173 & 0.0071 & 0.0 & 0.0224 \\
& Scd/Sd/Irr & 9 & 0.064 & 0.0506 & 0.0435 & 0.0602 \\
\hline SALT2 & E-S0 & 26 & 0.208 & -0.0686 & -0.0490 & 0.0411 \\
& S0a-Sc & 72 & 0.163 & -0.0196 & 0.0 & 0.0242 \\
& Scd/Sd/Irr & 14 & 0.108 & 0.0717 & 0.0913 & 0.0560 \\
\hline MLCS31 & E-S0 & 19 & 0.166 & -0.0187 & -0.0405 & 0.0432 \\
& S0a-Sc & 59 & 0.172 & 0.0218 & 0.0 & 0.0234 \\
& Scd/Sd/Irr & 9 & 0.118 & 0.1367 & 0.1149 & 0.0583 \\
\hline MLCS17 & E-S0 & 21 & 0.186 & -0.0521 & -0.0537 & 0.0416 \\
& S0a-Sc & 64 & 0.155 & 0.0016 & 0.0 & 0.0221 \\
& Scd/Sd/Irr & 10 & 0.109 & 0.1138 & 0.1122 & 0.0538 \\
\hline Average & E-S0 & & 0.182 & & -0.0536 & 0.0406 \\
& S0a-Sc & & 0.166 & & 0.0 & 0.0230 \\
& Scd/Sd/Irr & & 0.100 & & 0.0905 & 0.0571 \\
\hline Diff. & Scd-Irr-E-S0 & & & & 0.1441 & 0.0701 \\
\hline
\end{tabular}

Note. - For these results, SN Ia with $0.7 \leq \Delta \leq 1.2$ and $A_{V}>0.5$ have been excluded from MLCS31 and MLCS17. SN Ia with $-0.1 \leq c \leq 0.2$ from $\mathrm{SALT} / 2$ have been included. $\mathrm{N}$ is the number in each bin. RelativeWM is the weighted mean relative to the S0a-Sc bin. The Scd/Sd/Irr bin has the smallest average standard deviation while the E-S0 bin has the largest. The mean of Hubble residuals of the Scd/Sd/Irr hosts is greater by $2-\sigma$ than that of the E-S0 bin. 


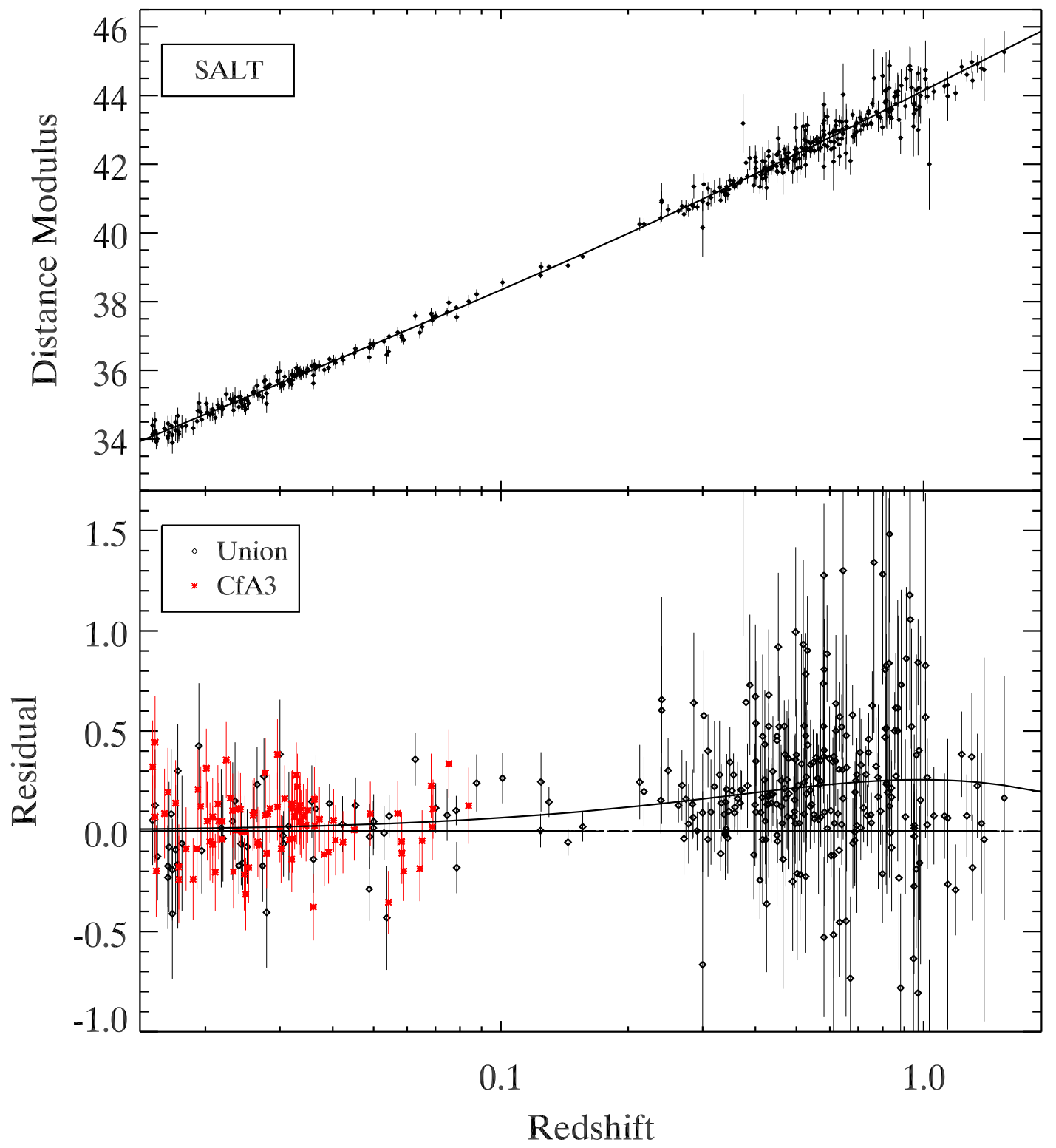

Fig. 1.- Hubble diagram and residuals for the Constitution sample fit by SALT. The bottom panel shows the new CfA3 SN Ia in red and the Union sample in black. The residuals are with respect to a universe without dark energy, $\Omega_{M}=0.27$ and $\Omega_{\Lambda}=0$. The best-fit cosmology is plotted in the residuals panels. The large scatter at high redshift is one of the main weaknesses of the conventional approach to calculating distance moduli from the SALT light-curve fits. 


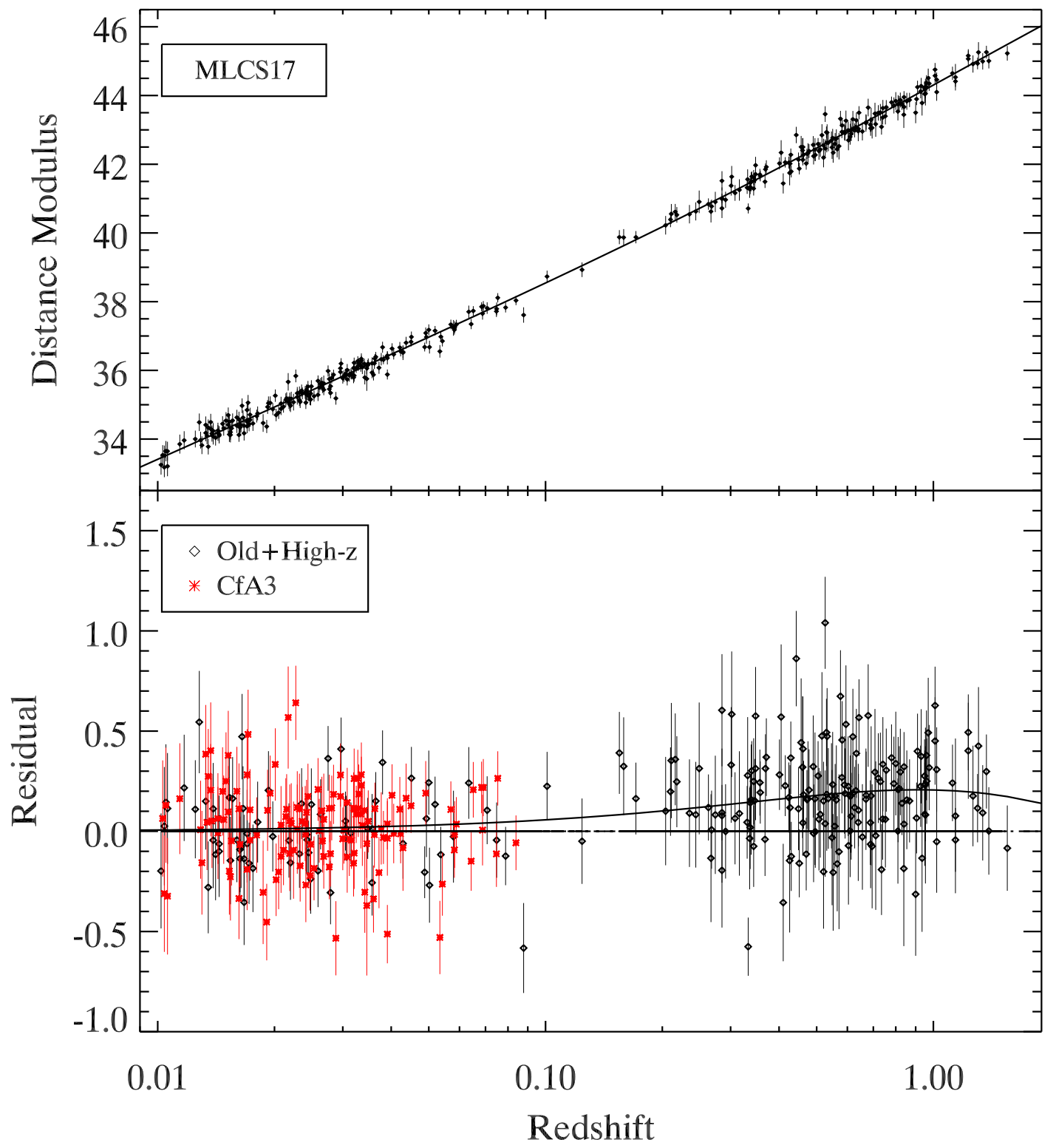

Fig. 2.- Hubble diagram and residuals for MLCS17. The new CfA3 points are shown in red and the OLD and High-z points are in black. MLCS17 (and MLCS31) has a smaller dispersion at high redshift than SALT (and SALT2). The nearby MLCS17 distances are larger than in SALT, making the High-z distances smaller relative to a matter-only universe and resulting in a greater value of $1+w$. This effect is seen in how the MLCS17 best-fit cosmology line is closer to the axis. 

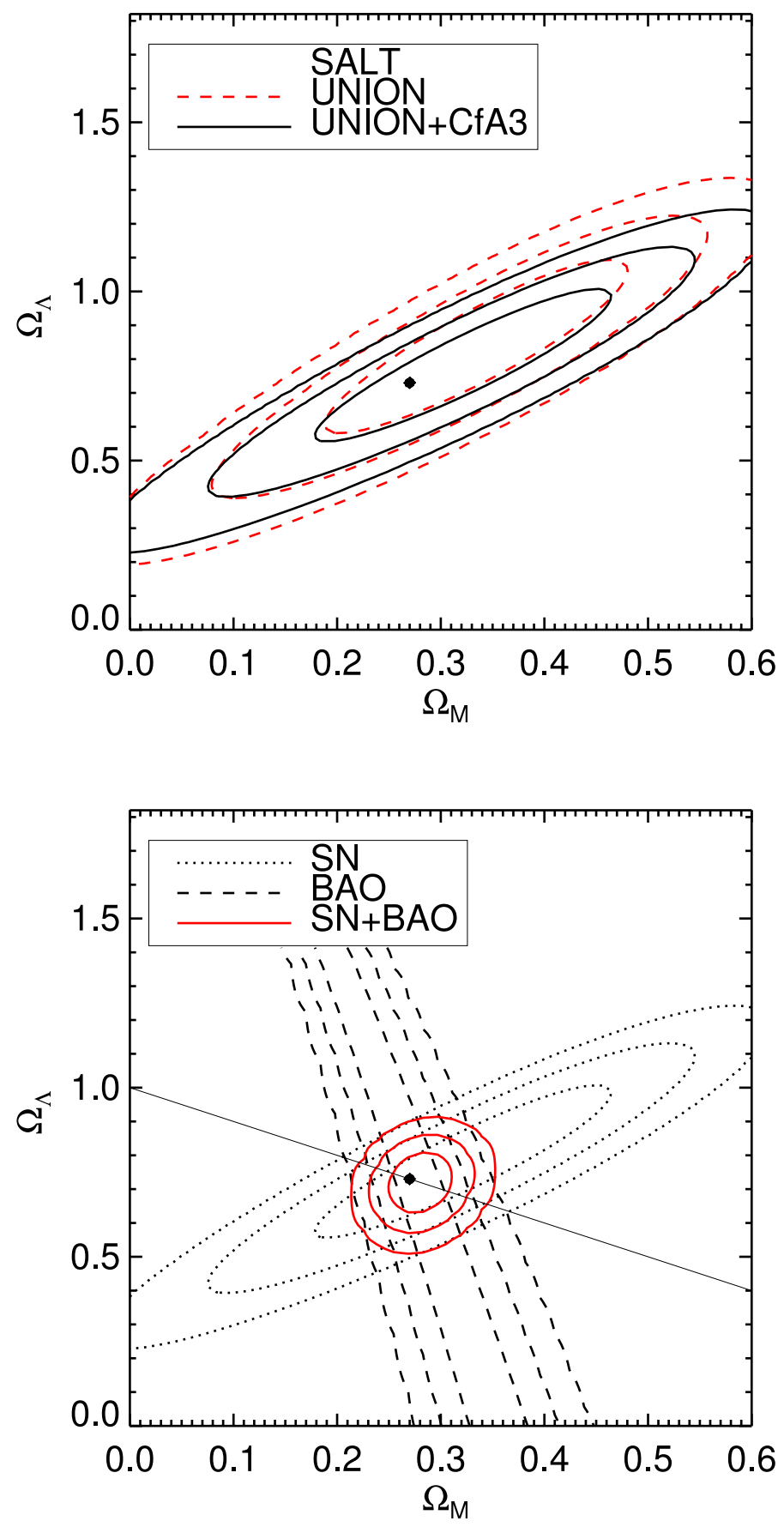

Fig. 3.- Contour plots of $\Omega_{\Lambda}$ vs. $\Omega_{M}$ for $1+w=0$ for SALT, with no assumptions about flatness. The concordance cosmology $\left(\Omega_{\Lambda}=0.73, \Omega_{M}=0.27\right)$ is shown as a dot. The top panel shows how adding the CfA3 sample considerably narrows the contours along the $\Omega_{\Lambda}$ axis. The bottom panel shows the combination of the SN contours with the BAO prior, with the flat-universe straight line overplotted for reference. 

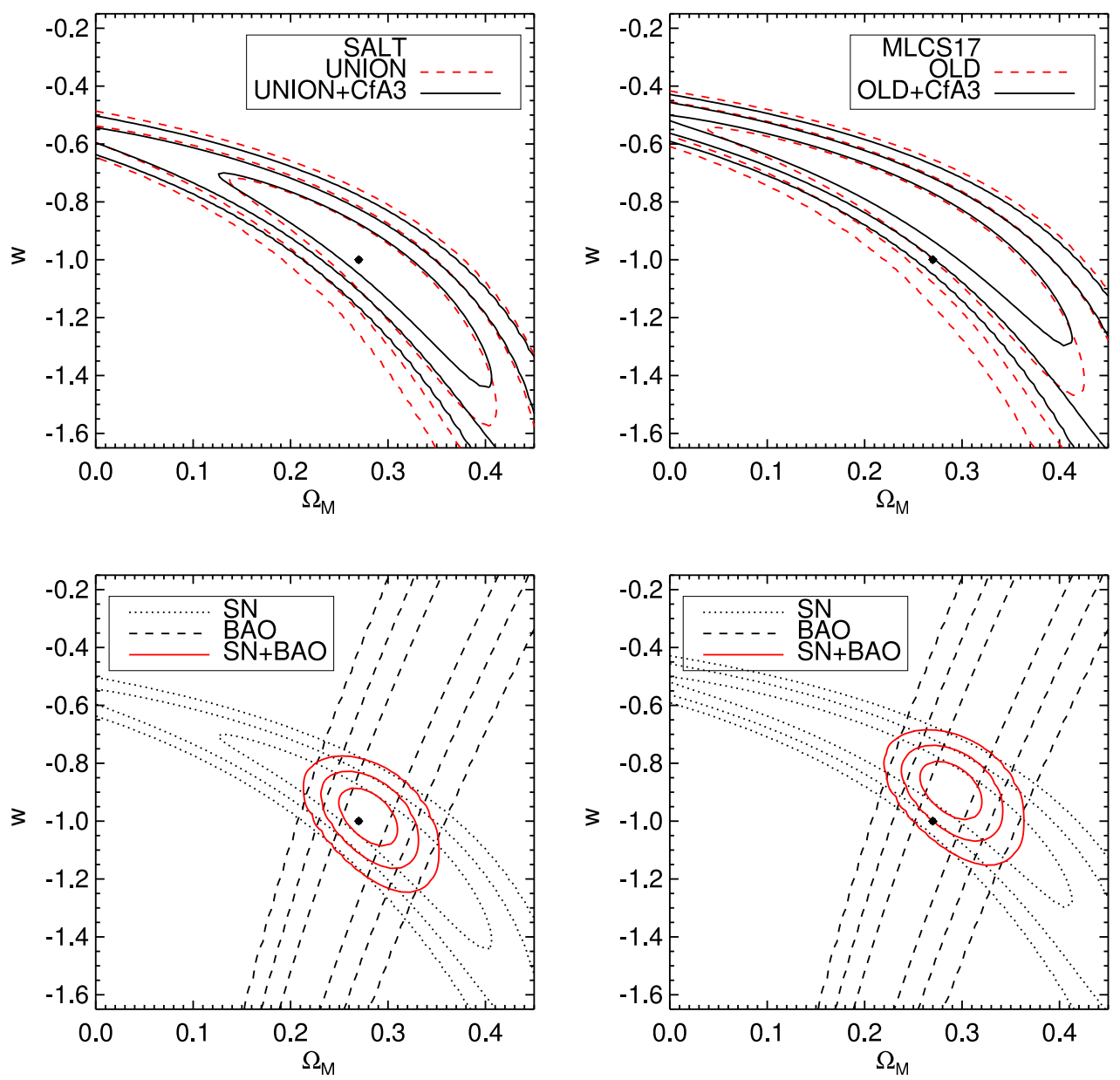

Fig. 4.- Contour plots of $w$ vs. $\Omega_{M}$ in a flat universe. The concordance cosmology $(w=-1$, $\left.\Omega_{\Lambda}=0.73, \Omega_{M}=0.27\right)$ is shown as a dot in each plot. The left two plots are for SALT while the right two plots are for MLCS17. The top row of plots show how adding the CfA3 sample considerably narrows the contours along the $w$ axis. The second row of plots show the combination of the SN contours with the BAO prior. 

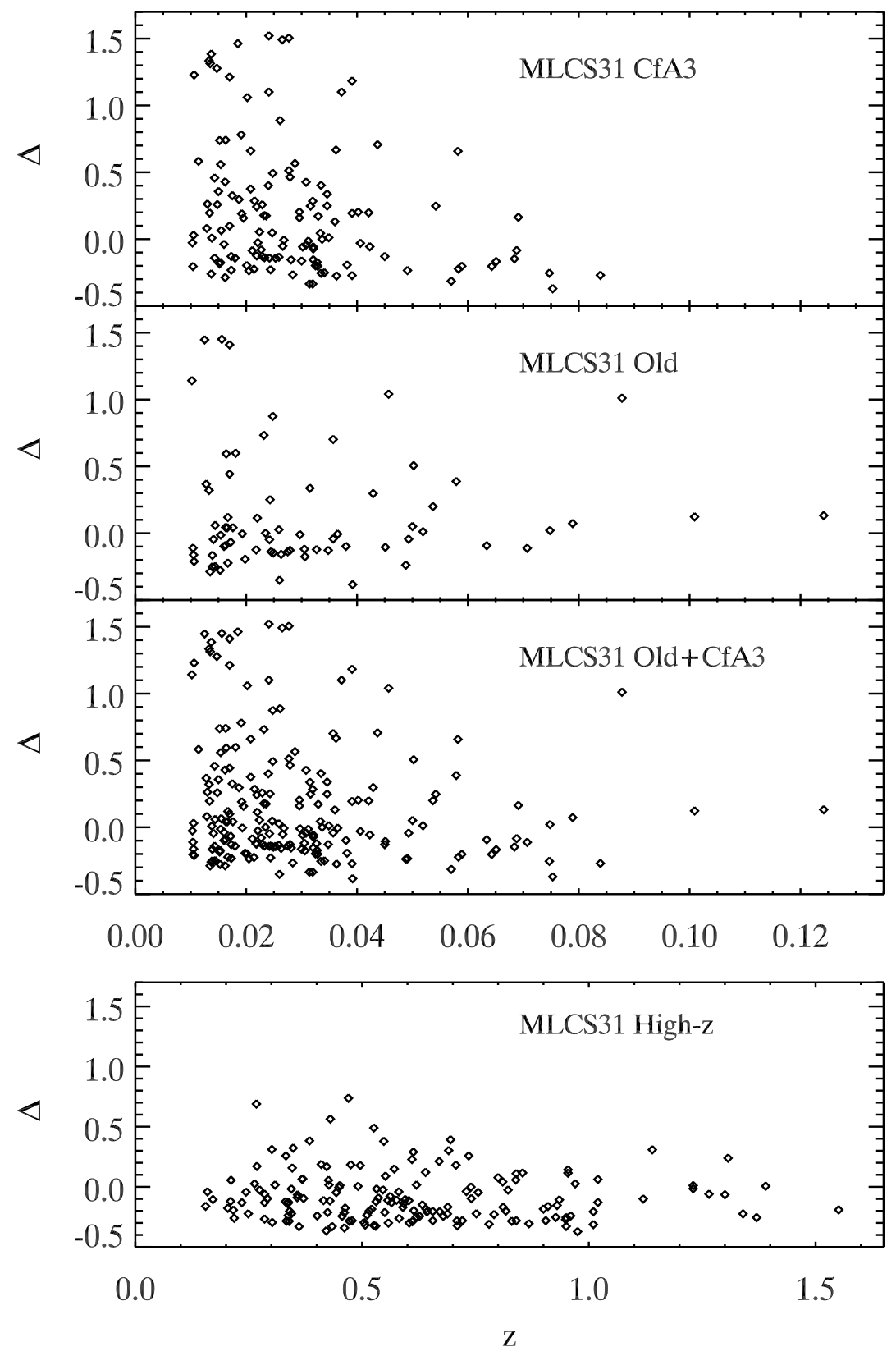

Fig. 5.- The distribution of $\Delta$ versus $z$. Faint SN Ia (high $\Delta$ ) are not found at higher redshifts because of magnitude-limited searches. The CfA3 sample has an effective limiting magnitude of $\sim 18.5$ mag. 

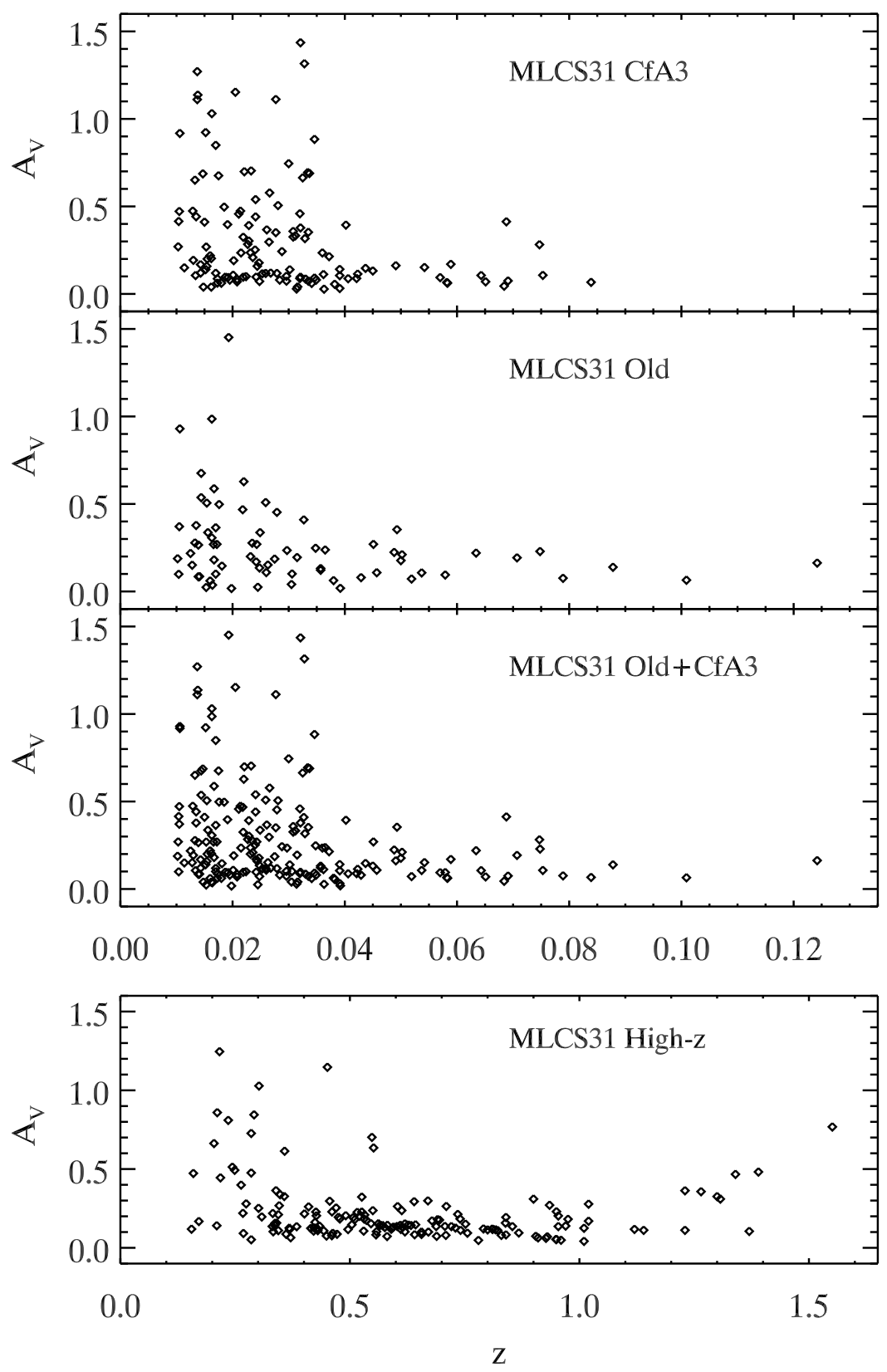

Fig. 6. - The distribution of $A_{V}$ versus $z$. Limiting-magnitude search effects reduce the number of highly extinguished SN Ia found at higher redshifts amongst all of the samples except for the Higher-Z, HST sample above $z \approx 1$, where some moderate-extinction objects are found. 

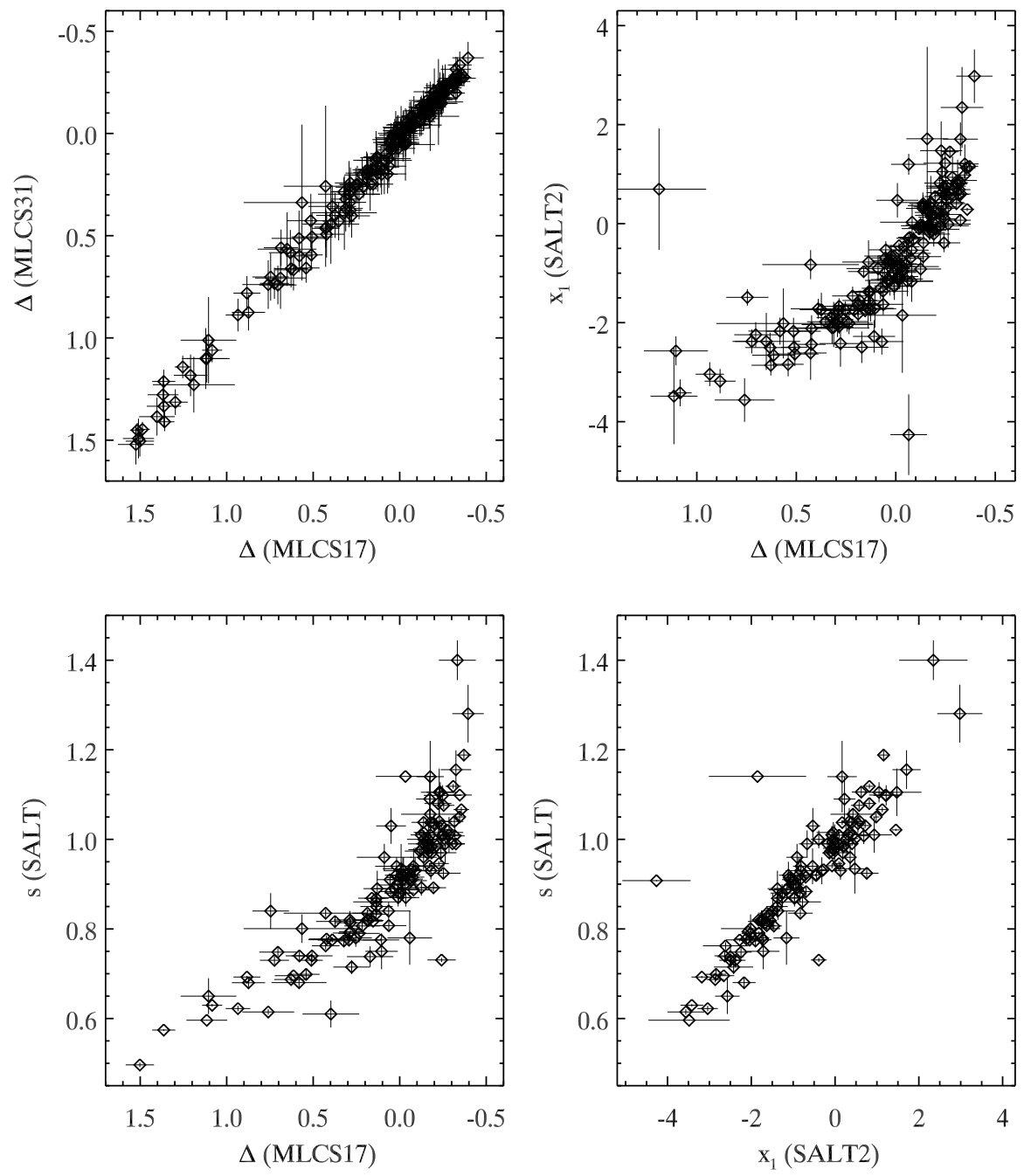

Fig. 7.- Relatively good correlation between light-curve shape parameters. The two biggest outliers in each of the upper-right and lower-right panels are due to three objects with relatively-poor SALT2 fits but good fits with other fitters. 

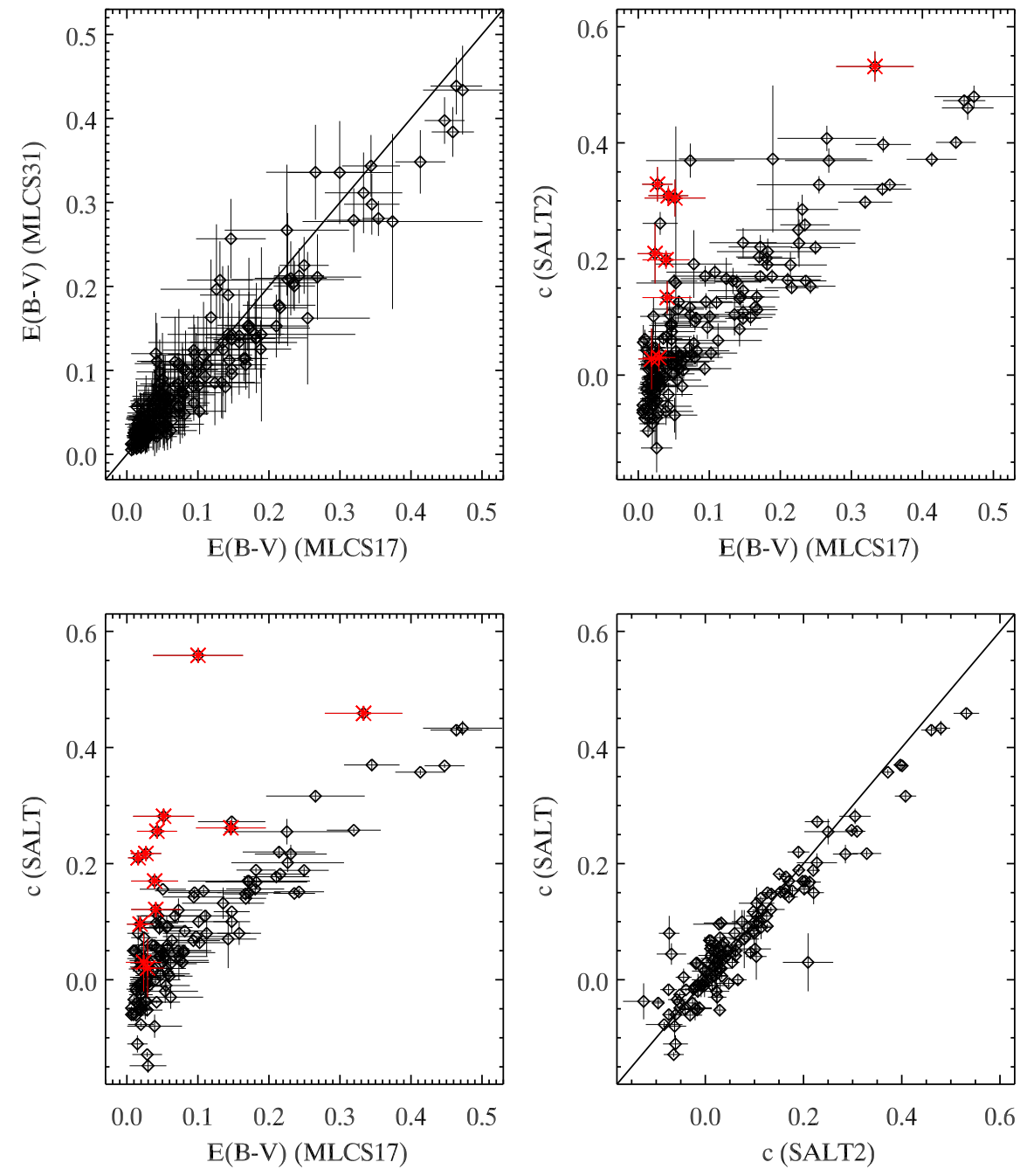

Fig. 8.- Good correlation between MLCS17 and MLCS31 host galaxy reddening: $E(B-$ $V)_{h o s t}=A_{V} / R_{V}$. Diagonal lines plotted in upper-left and lower-right panels to aid the eye. In SALT and SALT2, $c$ is a combination of intrinsic redness and host reddening. The red asterisks are intrinsically-red objects with $\Delta \geq 0.7$. The lower, diagonal boundary in the SALT/2 vs MLCS17 comparisons is the region where the SN Ia are intrinsically blue but suffer host reddening. There is good correlation between SALT/2 and MLCS17 for the objects that are intrinsically blue. The points above this lower edge are intrinsically redder and show that $c$ also measures intrinsic redness and cannot be directly compared with $E(B-V)$. 

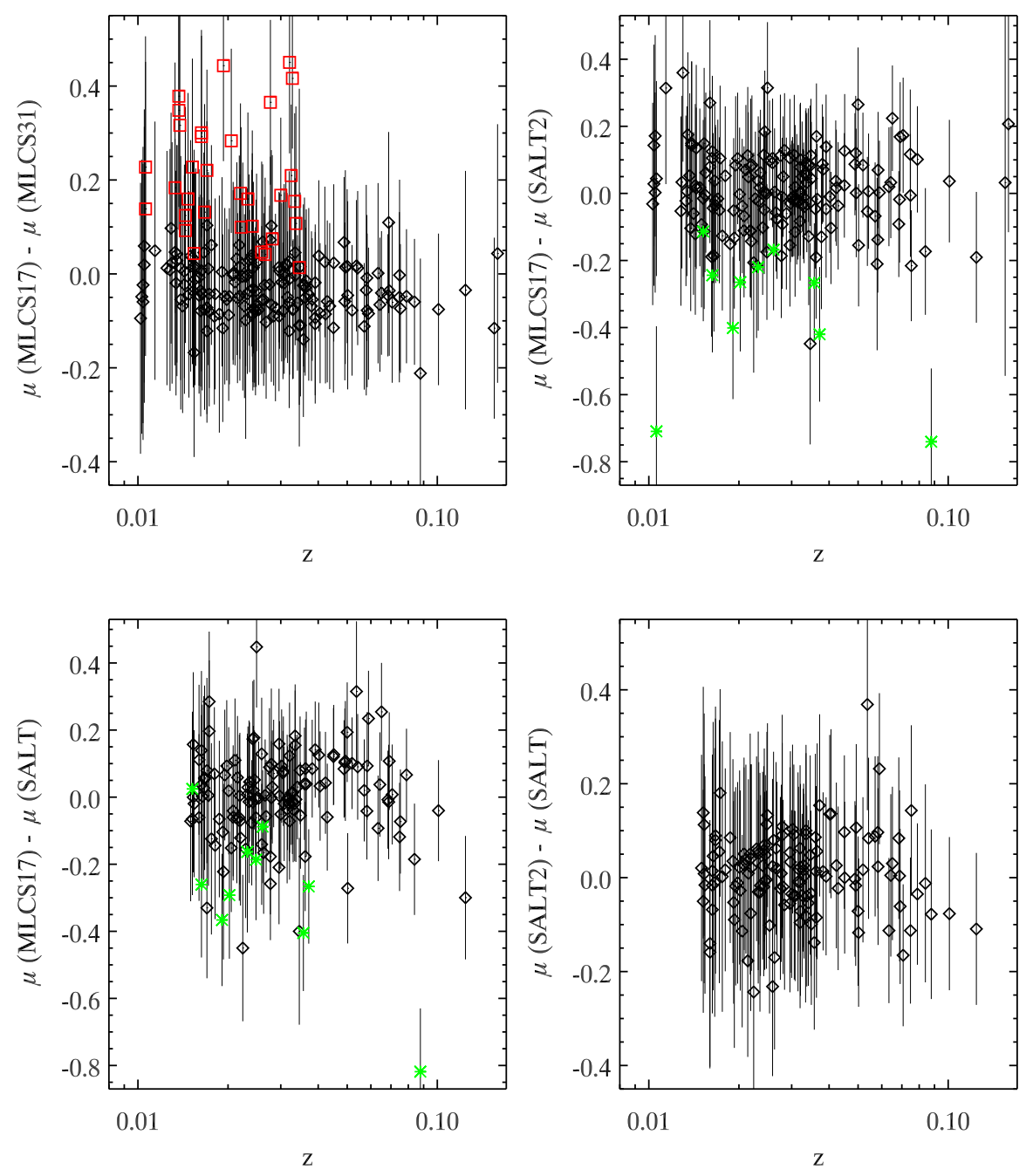

Fig. 9.- Difference in distance moduli between fitters versus redshift. The upper-left panel compares MLCS31 with MLCS17. The red squares in this panel have $A_{V}>0.5$ in MLCS31 and show a dramatic difference in distance from the MLCS17 values. The less-reddened SN Ia agree well. There might be a slight offset in the remaining values since the value of $\mu$ depends on the Hubble constant which was determined from the full samples. SALT/2 versus MLCS17 comparisons are good, especially when the problematic MLCS2k2 points with $0.7 \leq \Delta \leq 1.2$ in green are ignored. SALT and SALT2 agree well. 


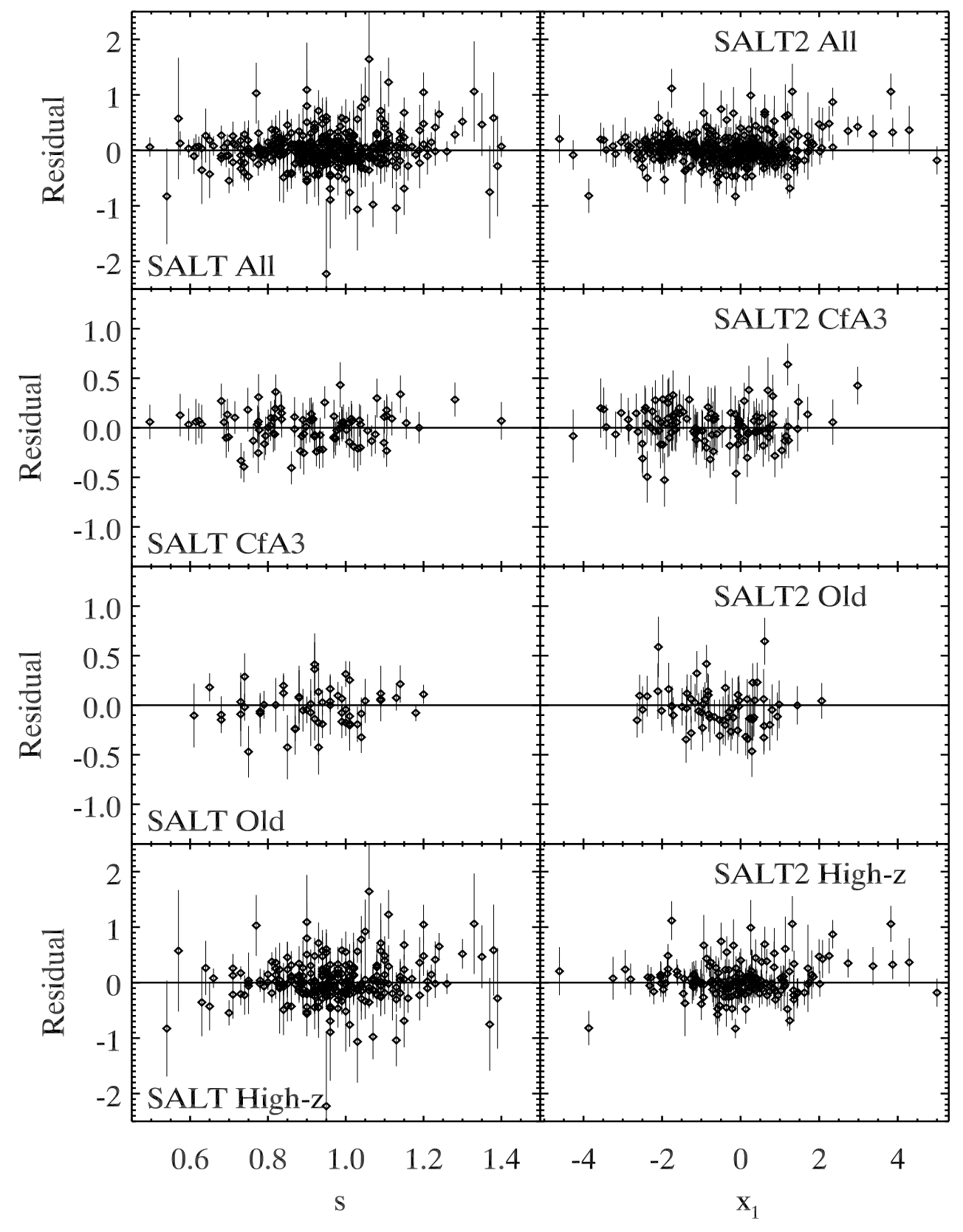

Fig. 10.- Hubble residuals relative to the best-fit cosmologies versus $s$ and $x_{1}$ for SALT and SALT2, respectively. 


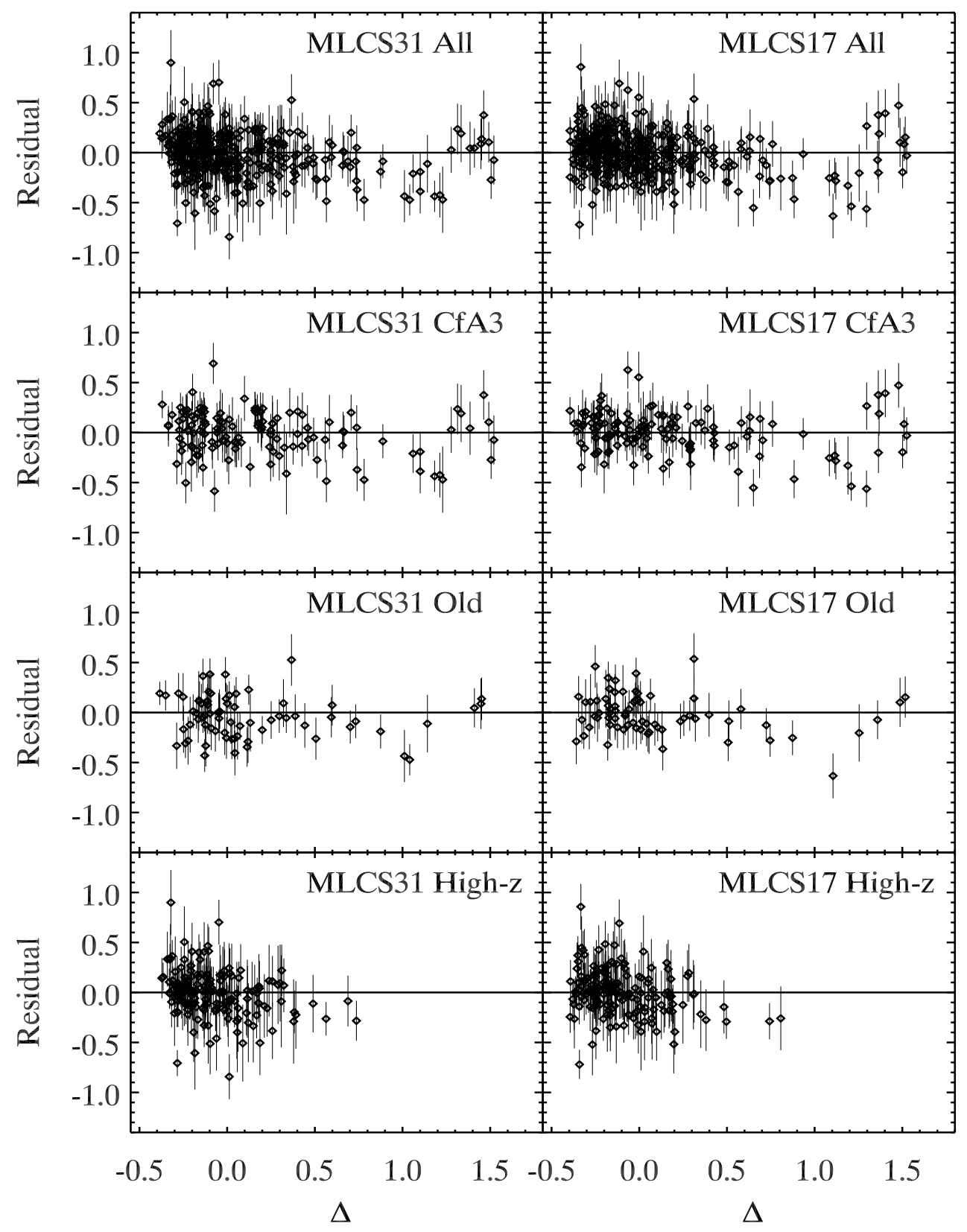

Fig. 11.- Hubble residuals relative to the best-fit cosmologies versus $\Delta$ for MLCS31 and MLCS17, respectively. There is a noticeable region of negative residuals between $0.7<\Delta<$ 1.2 . 

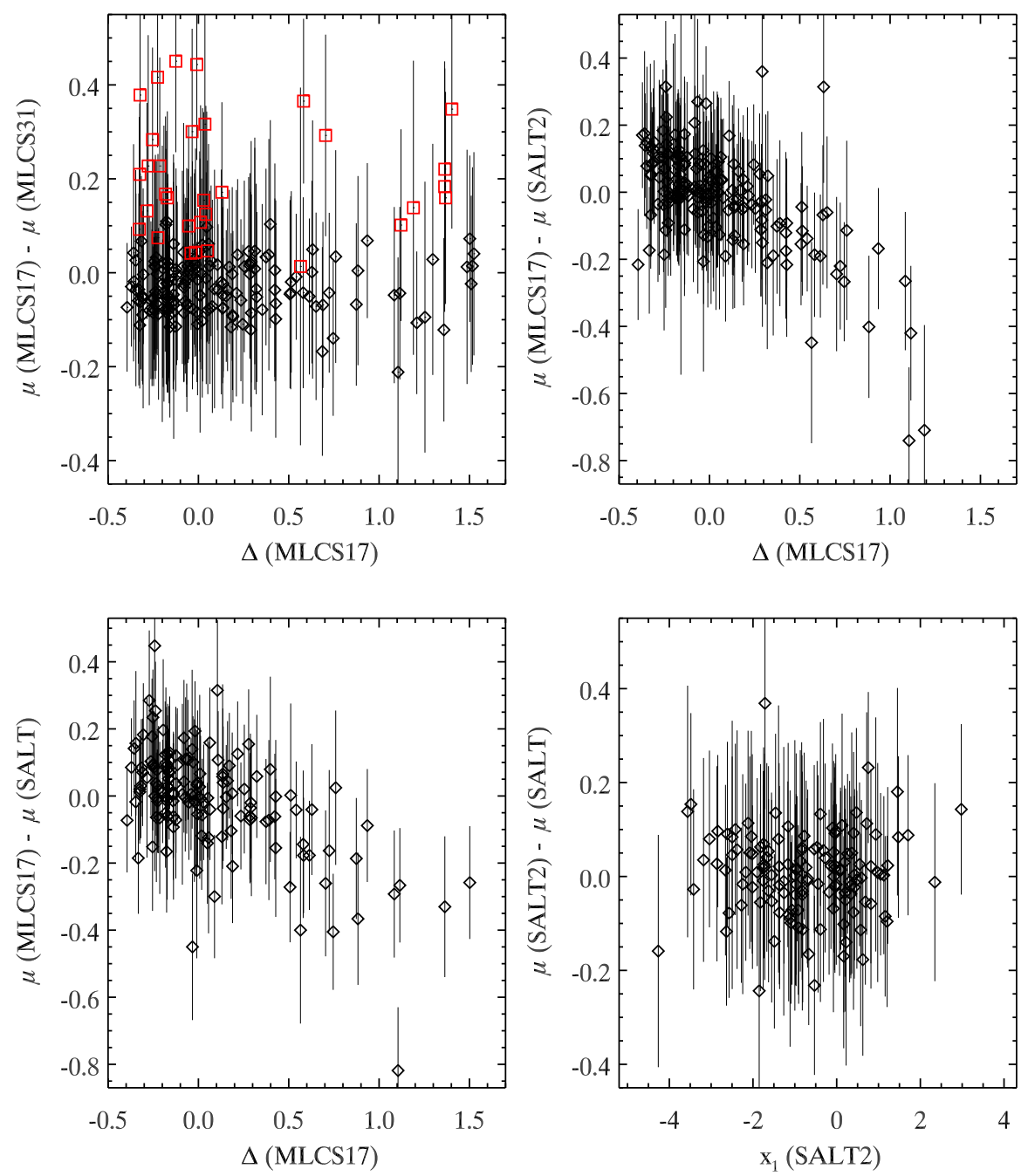

Fig. 12.- Comparison of distance moduli between the four fitters versus light-curve shape parameter. MLCS17 and MLCS31 agree well when the highly-reddened SN Ia (red squares) are ignored. SALT and SALT2 agree well. There is a systematic trend between MLCS2k2 and $\mathrm{SALT} / 2$, where fainter objects have relatively smaller distances, especially beyond $\Delta=0.7$. 

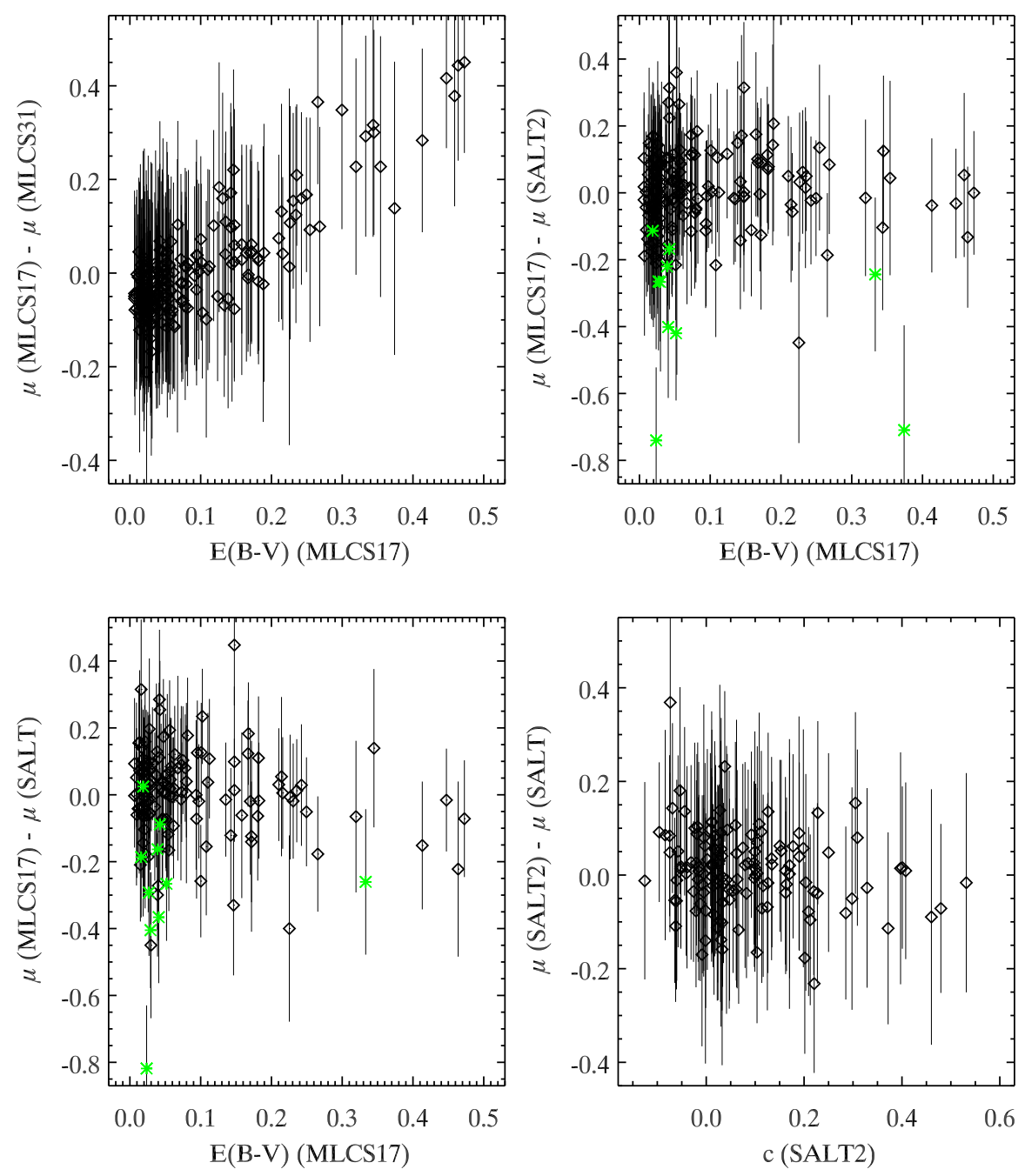

Fig. 13.- Comparison of distance moduli between the four fitters versus host-galaxy reddening in three panels and SALT2 $c$ in the fourth. The upper-left panel shows that MLCS31 (with $R_{V}=3.1$ ) probably overestimates the extinction, $A_{V}$, for highly-reddened objects while MLCS17 agrees well with SALT/2, especially when the green points $(0.7 \leq \Delta \leq 1.2)$ are ignored. SALT and SALT2 agree well. 


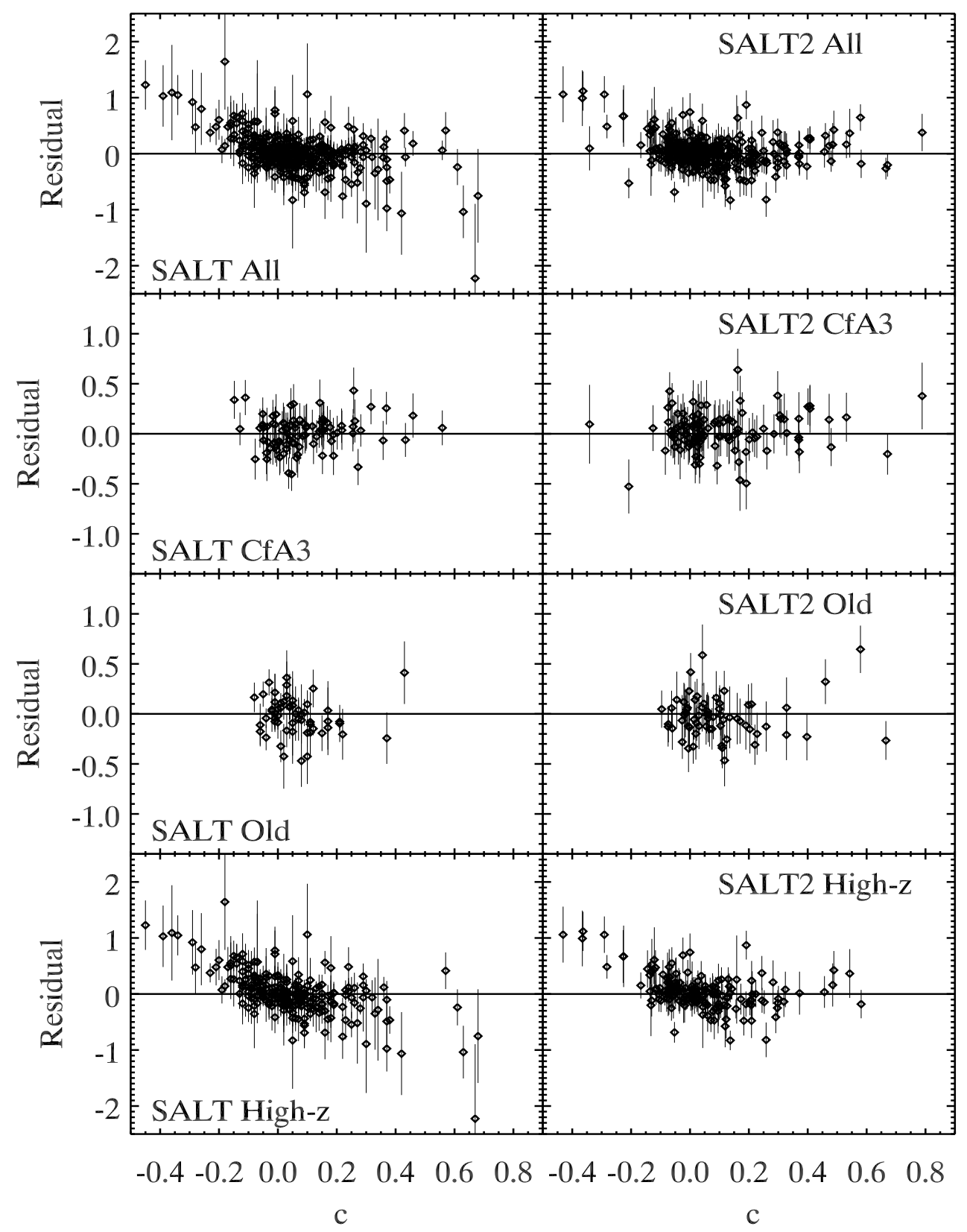

Fig. 14.- Hubble residuals relative to the best-fit cosmologies versus $c$ for SALT and SALT2, respectively. The nearby samples do not show any significant trends versus $c$ but the High-z sample does and we explore making a cut on $c$. 


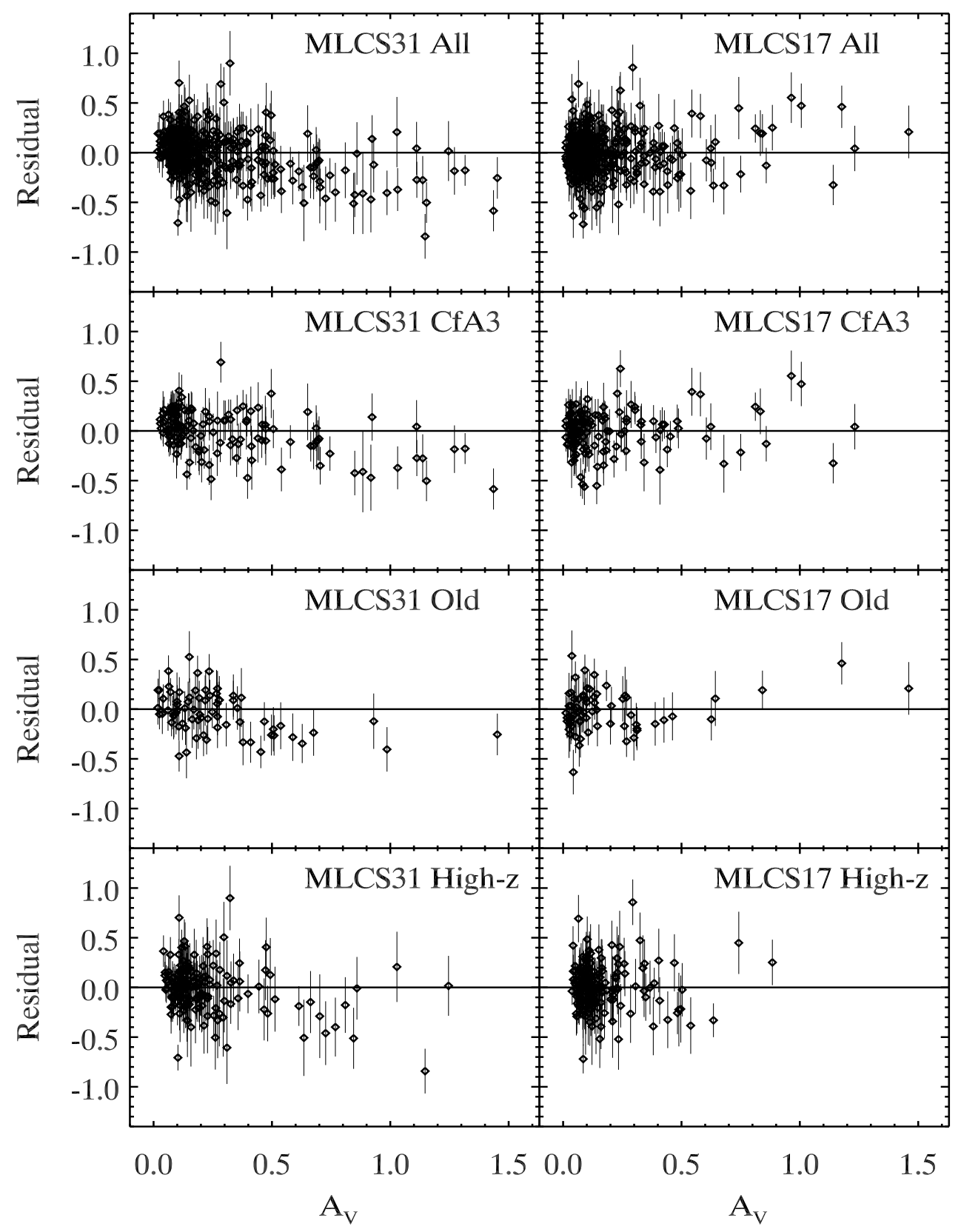

Fig. 15.- Hubble residuals relative to the best-fit cosmologies versus $A_{V}$ for MLCS31 and MLCS17, respectively. The residuals are noticeably negative beyond $A_{V} \approx 0.5$ for $R_{V}=3.1$, suggesting that $A_{V}$ is being overestimated by MLCS31. 


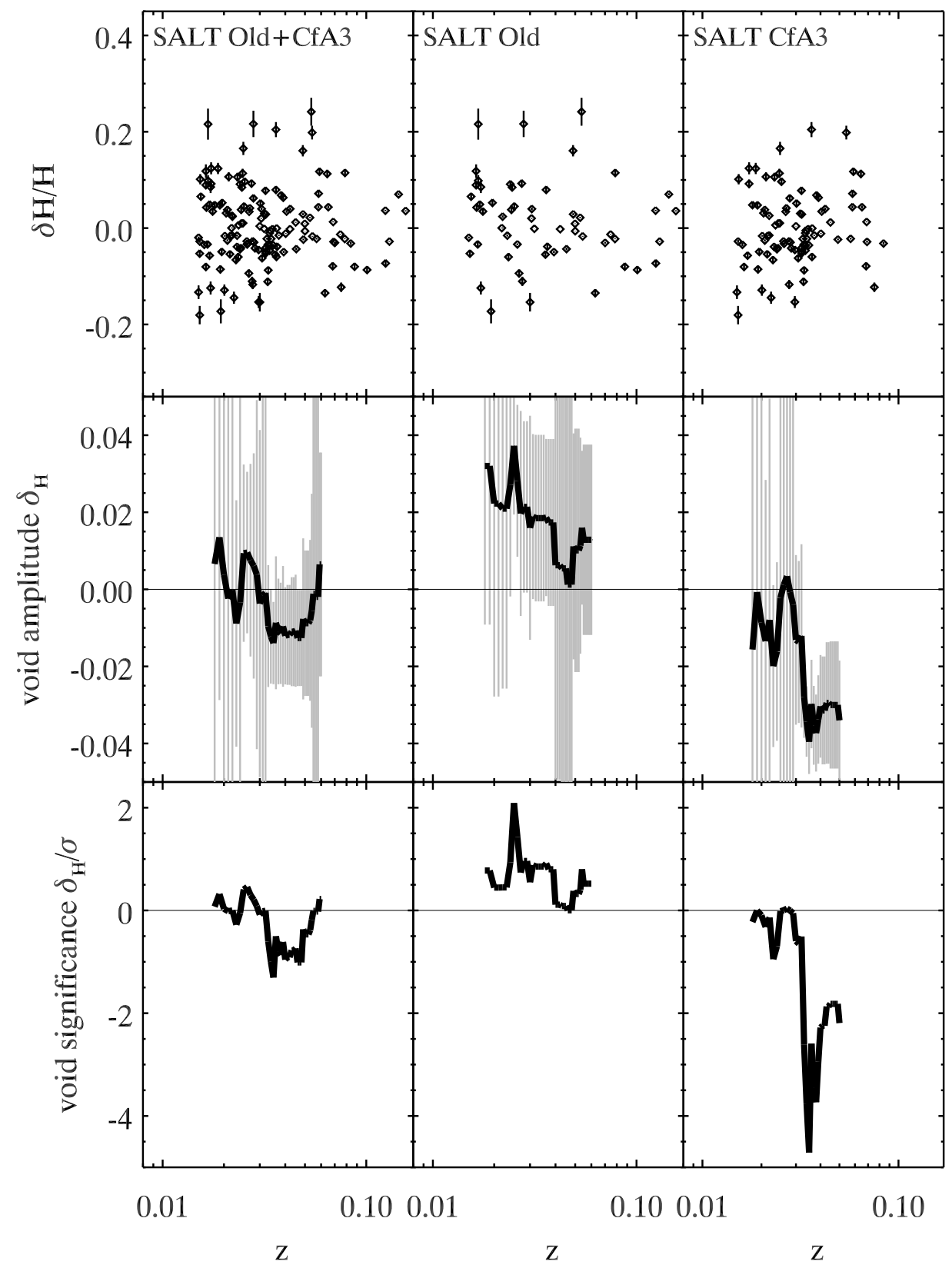

Fig. 16.- Hubble bubble for SALT. A negative but insignificant Hubble bubble is present in the OLD+CfA3 sample. 


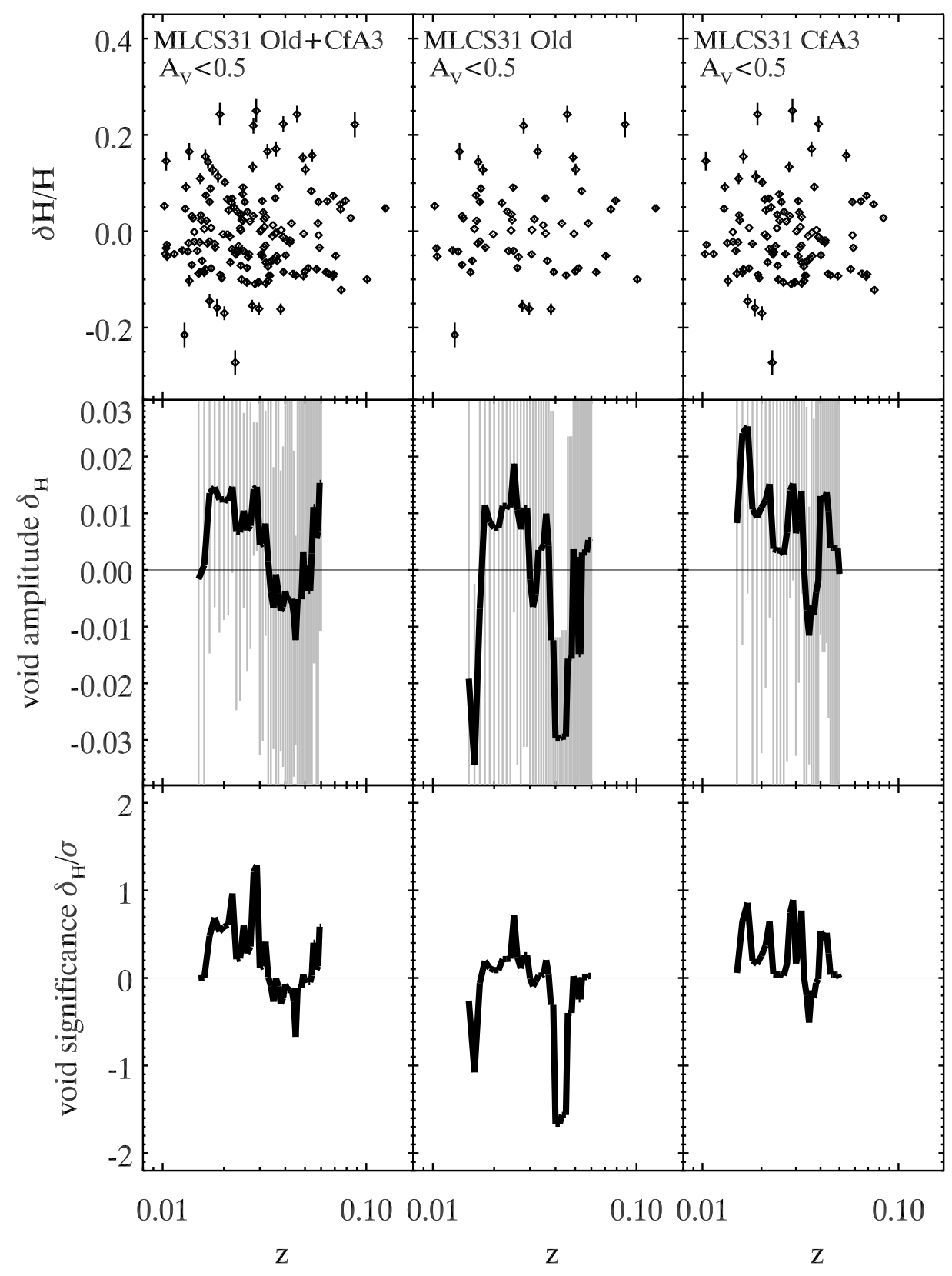

Fig. 17.- After a cut at $A_{V}=0.5$, the positive Hubble bubble for MLCS31 is now insignificant. 

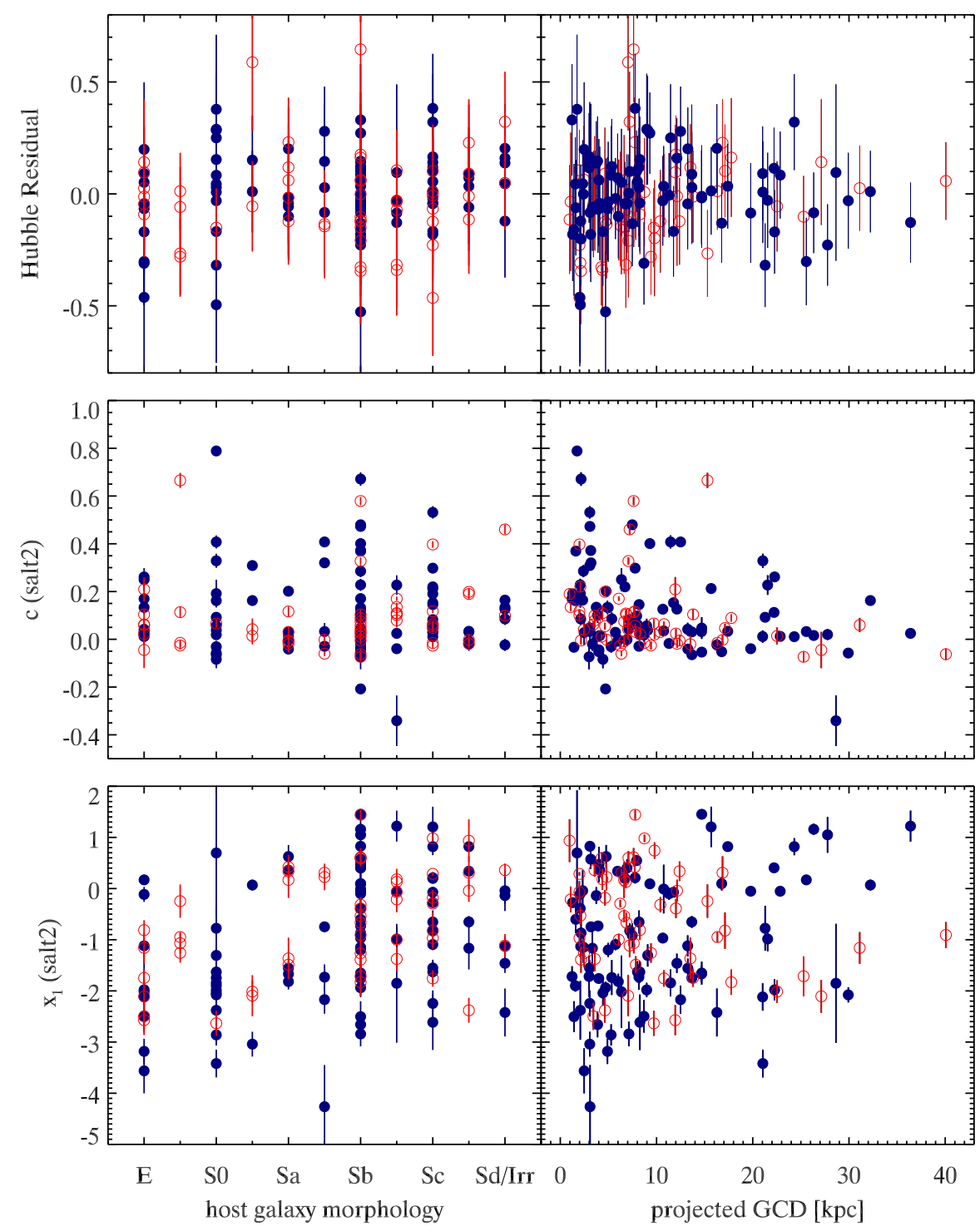

Fig. 18. - The top panels shows the SALT2 Hubble residuals versus host galaxy morphology and projected galactocentric distance. The middle panels plot SALT2 $c$ and the bottom panels plot stretch, $x_{1}$, versus morphology and PGCD. The Hubble residuals are more negative on average in the E/S0 hosts than in the Scd/Sd/Irr hosts by roughly $2 \sigma$. The Scd/Sd/Irr SN Ia show the smallest dispersion, although we caution that this may be due to small numbers of objects. The CfA3 sample adds many slow decliners, of great importance in matching the High-z sample as much as possible. Red empty circles are OLD and blue filled circles are CfA3. 

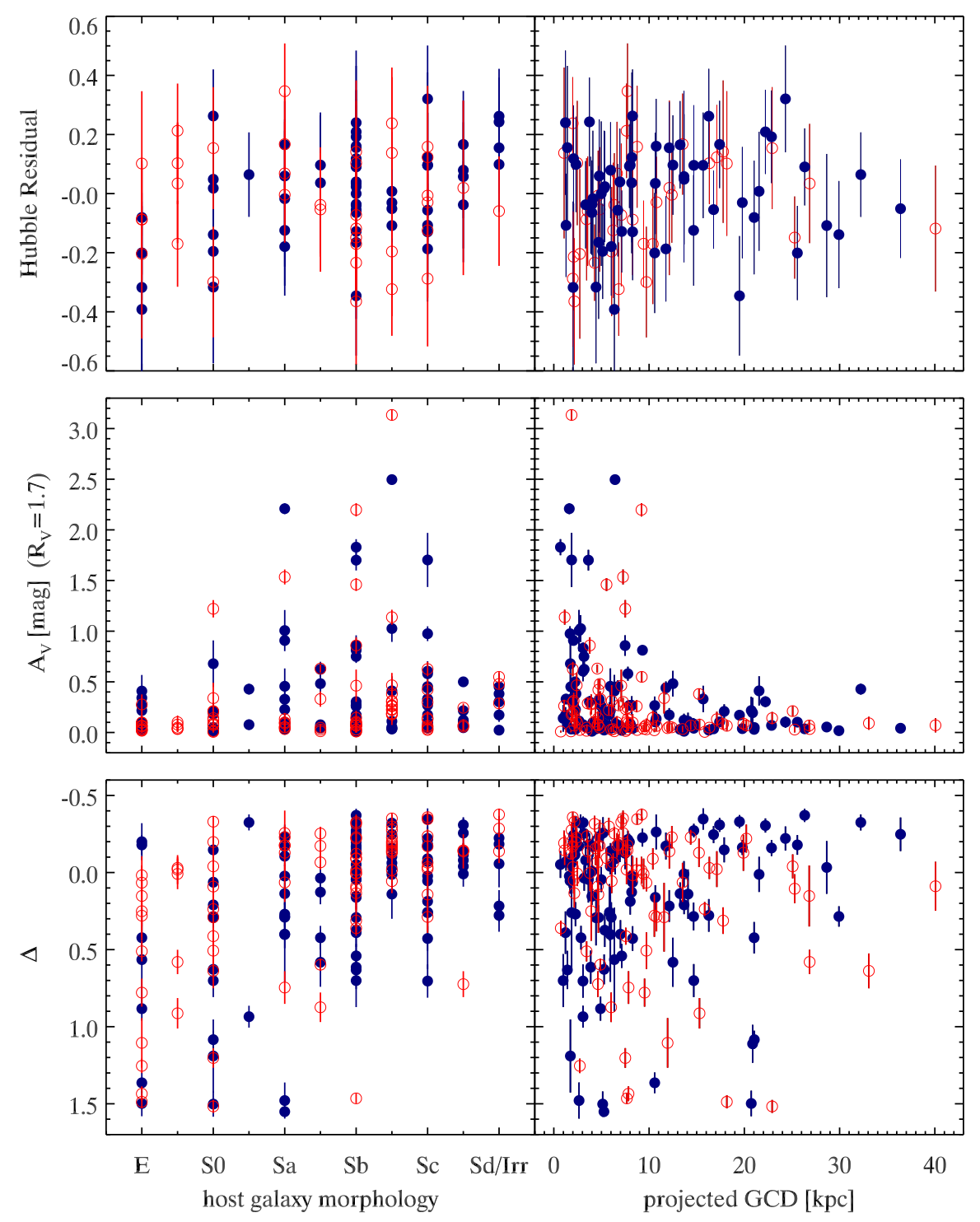

Fig. 19.- MLCS17 properties versus morphology and PGCD. Red empty circles are OLD and blue filled circles are CfA3. The negative residual SN Ia stand out in the E hosts. The same qualitative pattern is seen in MLCS17 as in MLCS31. Low host reddening for E/S0 and Scd/Sd/Irr SN Ia is seen. Highly reddened SN Ia do not occur beyond $\sim 10 \mathrm{kpc}$. Objects with $0.7 \leq \Delta \leq 1.2$ and $A_{V}>0.5$ have been removed from the Hubble residuals panels, so as to not influence possible trends. Two elliptical-host SN Ia have $\Delta \approx-0.2$. 


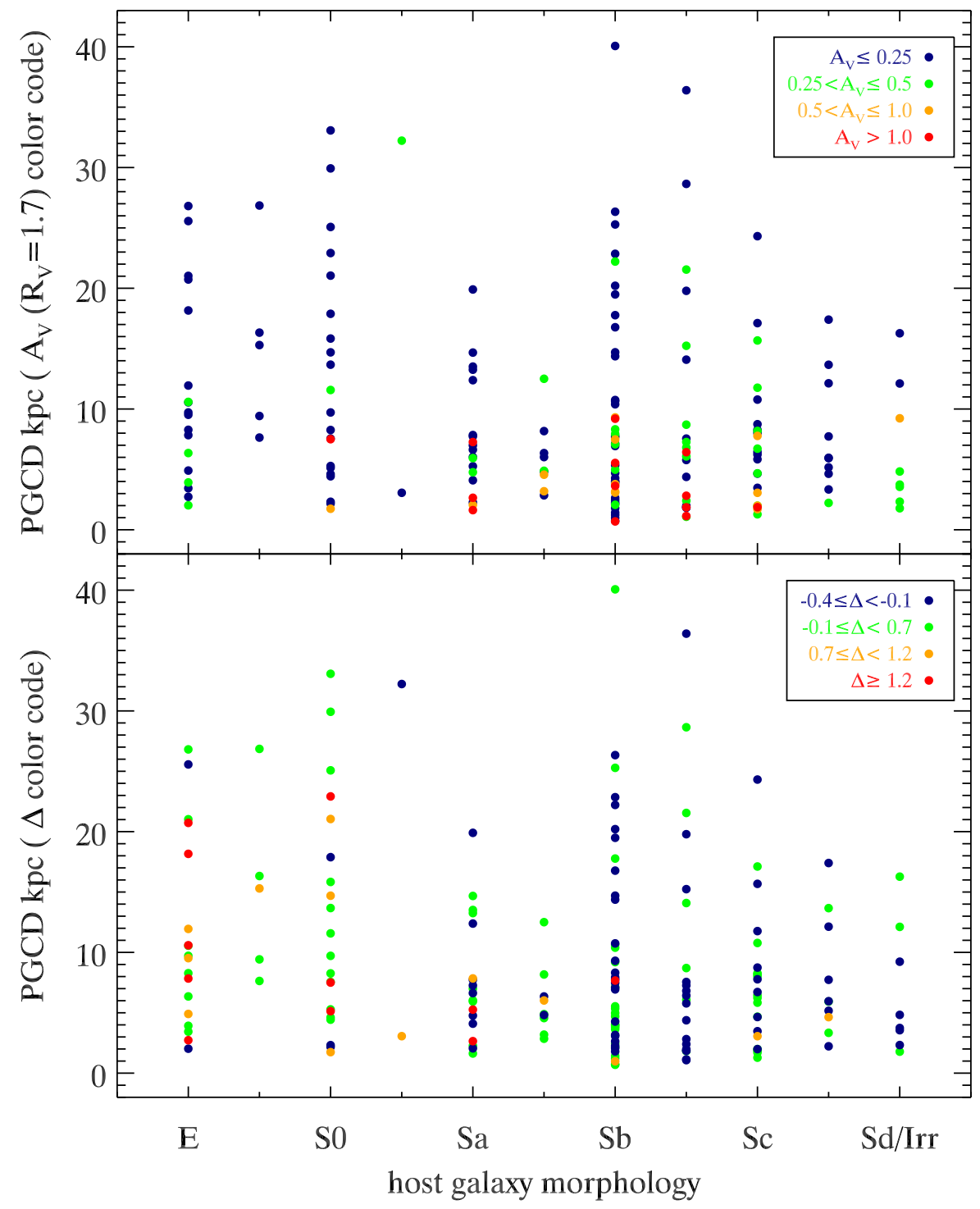

Fig. 20.- MLCS17 PGCD versus host galaxy morphology with dust extinction color coded in the top panel and $\Delta$ in the bottom panel. Top panel: blue $\left(A_{V} \leq 0.25\right)$, green $(0.25<$ $\left.A_{V} \leq 0.5\right)$, orange $\left(0.5<A_{V} \leq 1\right)$, red $\left(A_{V}>1\right)$. Bottom panel: blue $(-0.4 \leq \Delta<-0.1)$, green $(-0.1 \leq \Delta<0.7)$, orange $(0.7 \leq \Delta<1.2)$, red $(\Delta \geq 1.2)$. Light extinction in the inner regions of $\mathrm{Scd} / \mathrm{Sd} / \mathrm{Irr}$ hosts. High extinction is more prevalent in the $\mathrm{Sa} / \mathrm{Sb} / \mathrm{Sc}$ hosts, extending to highest PGCD in Sb hosts. Extinction is low in E hosts with "green" extinction $\mathrm{SN}$ Ia in the lower range of PGCD. 


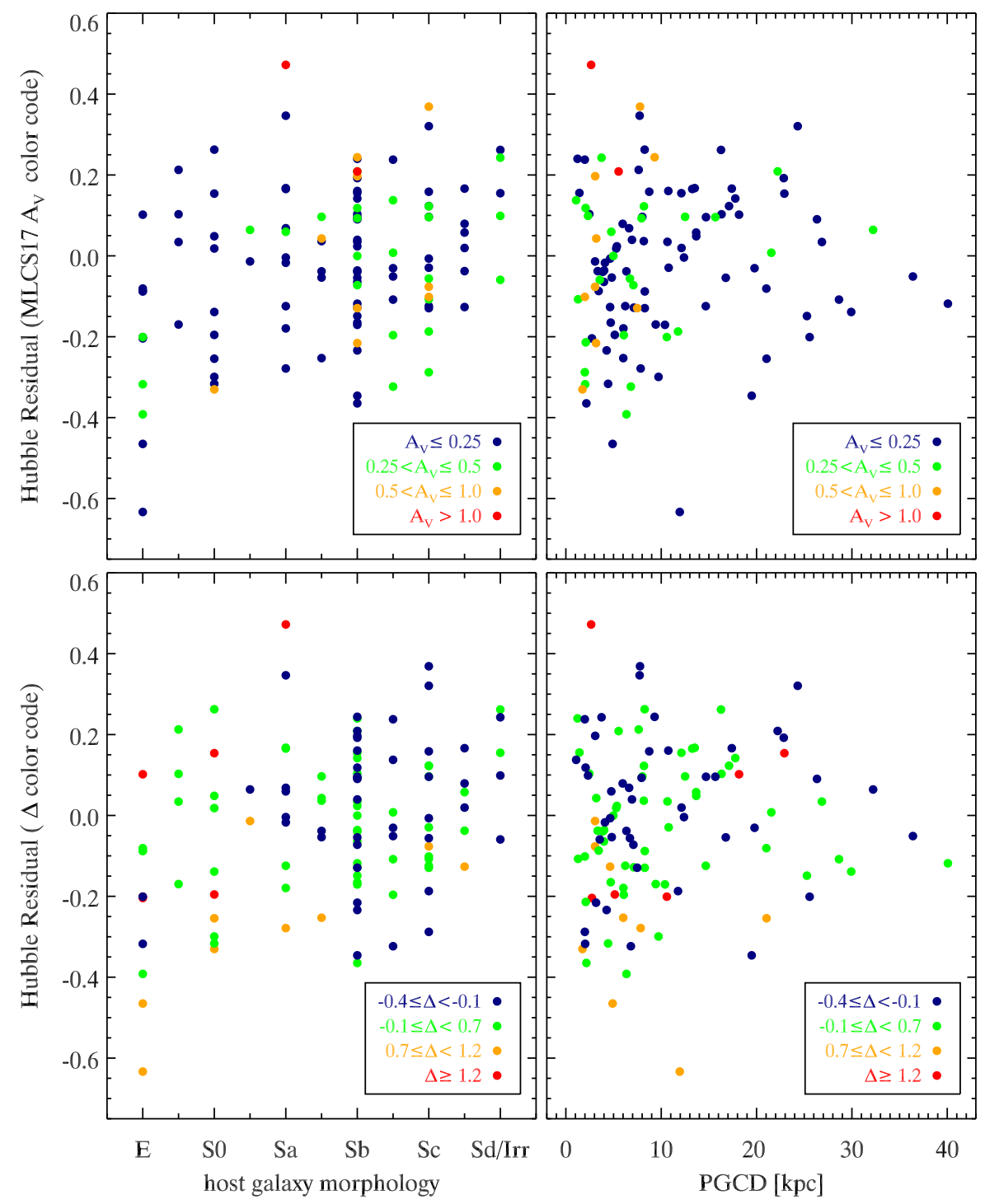

Fig. 21. - MLCS17 Hubble Residual versus morphology and PGCD. Same color coding as 20. Notice especially the bottom-left panel where the most-negative residual SN Ia in the E and S0 hosts are fast (orange), but not very-fast, decliners. These objects $(0.7 \leq \Delta<1.2)$ have been included in this plot to show that they are found across most morphological types and all have negative residuals. These orange points are excluded for our mean Hubble residual calculations of the three binned morphologies (E-S0, S0a-Sc, and Scd-Irr) and there is still $\mathrm{a} \sim 2 \sigma$ difference between the E-S0 and Scd-Irr mean Hubble residuals. 